\title{
U.S. ARMY RADIOLOGICAL BIOASSAY AND DOSIMETRY: THE RBD SOFTWARE PACKAGE
}

\author{
K. F. Eckerman, R. C. Ward ${ }^{1}$, and L. B. Maddox ${ }^{1}$ \\ 'Computing and Telecommunications Division \\ at Oak Ridge National Laboratory
}

Date Published: January 1993

Prepared for the U. S. Army

Army Materiel Command, Arlington, VA

under Interagency Agreement DOE No. 0883-1764-A1

Prepared by the

OAK RIDGE NATIONAL LABORATORY

Oak Ridge, Tennessee 37831

managed by

MARTIN MARIETTA ENERGY SYSTEMS, INC.

for the

U.S. DEPARTMENT OF ENERGY

under contract DE-AC05-84OR21400 


\section{CONTENTS}

ACKNOWLEDGMENTS $\ldots \ldots \ldots \ldots \ldots \ldots \ldots \ldots \ldots \ldots$

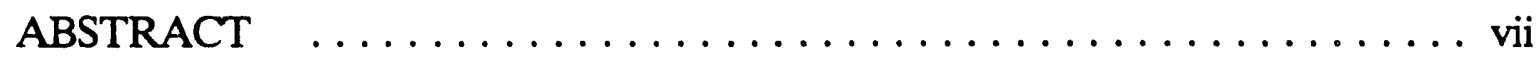

1. INTRODUCTION $\ldots \ldots \ldots \ldots \ldots \ldots \ldots \ldots \ldots \ldots \ldots \ldots$

2. INSTALLATION OF RBD SOFTWARE PACKAGE $\ldots \ldots \ldots \ldots \ldots 3$

3. GETTING STARTED WITH RBD $\ldots \ldots \ldots \ldots \ldots \ldots \ldots \ldots \ldots$

4. DESCRIPTION OF RBD SOFTWARE PACKAGE $\ldots \ldots \ldots \ldots \ldots 13$

5. RBD SOFTWARE TUTORIAL $\ldots \ldots \ldots \ldots \ldots \ldots \ldots \ldots \ldots \ldots \ldots$

5.1 Modifying the site-specific file SITE.DFT $\ldots \ldots \ldots \ldots \ldots \ldots \ldots 34$

5.2 Entering bioassay data into a bioassay data base $\ldots \ldots \ldots \ldots \ldots 36$

5.3 Browsing records in a bioassay data base $\ldots \ldots \ldots \ldots \ldots \ldots 45$

5.4 Extracting records from a bioassay data base and computing intake and dose using RBDCOMP ........... 49

5.5 Importing records into a bioassay data base using AUTOLOAD . . . 62

5.6 Importing RBDCOMP output into an output data base $\ldots \ldots \ldots 65$

5.7 Browsing records in an output data base $\ldots \ldots \ldots \ldots \ldots \ldots 66$

5.8 Entering exposure history records into a bioassay data base, extracting records, and computing intake and dose $\ldots \ldots 68$

5.9 Using the measurement and intake windows in computing

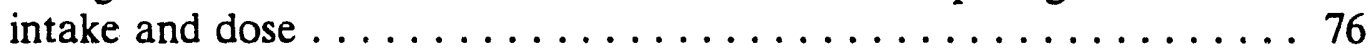

5.10 Generating a committed dose report using REPORT $\ldots \ldots \ldots \ldots 80$

6. DETAILS OF OPERATION OF RBD PROGRAMS AND UTILITIES .. 83

7. ALGORITHMS FOR ESTIMATING INTAKE OF RADIONUCLIDES .. 95

8. TECHNICAL DESCRIPTION OF NUCLIDE DATA FILES $\ldots \ldots \ldots 111$

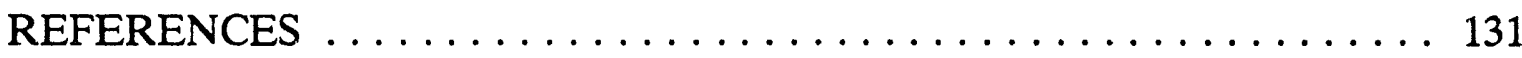

Appendix A: FORMAT OF BIOASSAY RECORDS $\ldots \ldots \ldots \ldots \ldots$

Appendix B: FORMAT OF OUTPUT RECORDS . . . . . . . . . . . 139 
Appendix C: FORMAT OF EFFECTIVE COMMITTED DOSE RECORDS 143

Appendix D: CONTENTS OF SITE.DFT FILE $\ldots \ldots \ldots \ldots \ldots \ldots \ldots$

Appendix E: EASY MODE OF OPERATION OF RBD $\ldots \ldots \ldots \ldots 147$ 


\section{ACKNOWLEDGMENTS}

The authors wish to thank the following individuals who, as members of the RBD Review Committee, provided guidance in the development of the RBD software.

R. K. Bhat, Chairman, US Army Belvoir RDE Center, Belvoir, VA.

A. Edward Abney, US Army Ionizing Radiation Dosimetry Center, Lexington, KY.

LTC C. A. Day, US Army Materiel Command, Alexandria, VA.

MAJ S. Dunston, USAEHA, Aberdeen Proving Ground, MD.

P. A. Elker, US Army Materiel Command, Alexandria, VA.

L. A. Slaback, National Institute of Standards and Technology, Gaithersburg, MD.

R. J. Swatski, USAEHA, Aberdeen Proving Ground, MD.

W. R. Webber, National Institute of Standards and Technology, Gaithersburg, MD.

The constructive criticism and suggestions of the review committee, chaired by Dr. Bhat, were invaluable to the RBD project. The authors are also indebted to Dr. Bhat for his support and encouragement throughout the work. In addition, this project also benefitted from the comments and suggestions of a Martin Marietta Energy Systems' Committee on the DOSEXPRT project. Shortfalls in the RBD software are attributable to the authors; the success we all share.

The authors would also like to express their appreciation to Sonia Rogers for her attention to the details in preparation of this manuscript and to Mark Cristy, Andrea Sjoreen, and Catherine Shappert for attentive and careful editing of the manuscript.

This work was funded by the U.S. Army Belvoir Research and Development Center under contract A6488 with Martin Marietta Energy System, Inc., and Interagency Agreement DOE No. 0883-1764-A1 with the U.S. Department of Energy. 


\begin{abstract}
The RBD (Radiological Bioassay and Dosimetry) software package was developed for the U. S. Army Materiel Command, Arlington, Virginia, to demonstrate compliance with the radiation protection guidance 10 CFR Part 20 (ref. 1). Designed to be run interactively on an IBM-compatible personal computer, RBD consists of a data base module to manage bioassay data and a computational module that incorporates algorithms for estimating radionuclide intake from either acute or chronic exposures based on measurement of the worker's rate of excretion of the radionuclide or the retained activity in the body. In estimating the intake, RBD uses a separate file for each radionuclide containing parametric representations of the retention and excretion functions. These files also contain dose-per-unit-intake coefficients used to compute the committed dose equivalent. For a given nuclide, if measurements exist for more than one type of assay, an auxiliary module, REPORT, estimates the intake by applying weights assigned in the nuclide file for each assay. Bioassay data and computed results (estimates of intake and committed dose equivalent) are stored in separate data bases, and the bioassay measurements used to compute a given result can be identified. The REPORT module creates a file containing committed effective dose equivalent for each individual that can be combined with the individual's external exposure.
\end{abstract}




\section{INTRODUCTION}

The general principles of monitoring for the radiation protection of workers have been set out in various reports of the International Commission on Radiological Protection (ICRP) $)^{2-5}$ and the National Council on Radiation Protection and Measurements (NCRP). ${ }^{6}$ Monitoring of individuals for internal exposure is based on the measurement of radionuclides in the whole body (or in specific organs/regions) and on the measurement of radionuclides in excreta. For quantitative assessment of internal exposure the results of these measurements must be translated into quantities that can be compared with the primary radiation protection guidance (limits on committed effective dose equivalent or committed dose equivalent to specific organs) or with the secondary quantities (Annual Limits of Intake - ALI). ${ }^{5}$ The relationships between levels in the body or in excreta and the estimated intake and resulting dose can only be established through the use of biokinetic and dosimetric models describing the behavicr of the material in the body.

Interpretation of bioassay measurements in terms of the primary and secondary radiation protection quantities often requires considerable computational effort, thus necessitating the use of a computer. For radionuclides that are tenaciously retained in the body, the interpretation of contemporary measurements requires the information of the complete exposure history of the individual to that nuclide. Thus the management of the bioassay data aiso necessitates use of a computer.

The RBD (Radiological Bioassay and Dosimetry) software package was developed for the U.S. Army Materiel Command, Arlington, Virginia, by Keith F. Eckerman, Richard C. Ward, and Linda B. Maddox of Oak Ridge National Laboratory and Martin Marietta Energy Systems. This documentation is for Version 4.1 of the RBD software. The RBD software was designed to be run interactively on an IBM-compatible personal computer ( 80286 or higher CPU) with $640 \mathrm{~K}$ of memory and a hard disk. Graphical output is available on systems with CGA, EGA, VGA, and HERCULES graphics adapters and color or monochrome monitors. Presently, the RBD computational module requires $412 \mathrm{~K}$ of memory. An additional $50 \mathrm{~K}$ of memory is required for the fonts used on the retention/excretion plots. A math co-processor is not required, but is highly recommended and will be used by RBD if present. The entire RBD software package requires just over 3.2 Megabytes of disk space at the time of installation. Additional space must be available for the files created by RBD.

The RBD software package is intended to serve as a tool for the U.S. Army in demonstrating compliance with occupational radiation protection regulations as given in 10 CFR Part 20 (ref. 1). RBD consists of data base tools to manage bioassay data and a computational program that incorporates algorithms for estimating radionuclide intake from either acute or chronic exposures based on measurement of the worker's rate of excretion of the radionuclide or the retained activity in the body. The algorithms use contemporary dosimetric and biokinetic data in the interpretation of the bioassay measurernents. In formulating the algorithms and developing the software, the needs of quality assurance and the desire to limit future maintenance costs were considered. 
In estimating the intake, the RBD code uses a separate paraineter file for each radionuclide. Each radionuclide parameter file contains information for computing intake and dose and information to control the progress of the computations. To date, 41 radionuclide data files have been created:

$\begin{array}{lllllllll}\text { H-3 } & \text { C-11 } & \text { C-14 } & \text { Na-22 } & \text { Na-24 } & \text { P-32 } & \text { Co-57 } & \text { Co-58 } & \text { Co-60 } \\ \text { Ni-63 } & \text { Sr-85 } & \text { Sr-89 } & \text { Sr-90 } & \text { Tc-99 } & \text { Tc-99M } & \text { I-125 } & \text { I-129 } & \text { I-131 } \\ \text { I-135 } & \text { Cs-134 } & \text { Cs-137 } & \text { Pm-147 } & \text { Eu-152 } & \text { Eu-154 } & \text { Eu-155 } & \text { Tl-201 } & \text { Ra-224 } \\ \text { Ra-226 } & \text { Th-228 } & \text { Th-232 } & \text { U-234 } & \text { U-235 } & \text { U-236 } & \text { U-238 } & \text { U-NAT } & \text { Pu-238 } \\ \text { Pu-239 } & \text { Pu-241 } & \text { Am-242 } & \text { Cm-242 } & \text { Cm-244 } & & & & \end{array}$

The nuclide data files are combined into a single binary, direct-access file that will be updated by the authors of $\mathrm{RBD}$ whenever new radionuclides are added.

The nuclide data files and the implementation of the algorithms in a similar code (DOSEXPRT) ${ }^{7}$ developed for Martin Marietta Energy Systems have undergone validation and verification testing by an outside contractor. In addition, individuals within Martin Marietta Energy Systems who were not connected with the development of RBD have examined the logic of the computational algorithms.

Section 2 contains the instructions for installing the RBD software on an IBM-compatible personal computer ( 80286 or higher CPU). This is followed by Sect. 3, which contains directions on getting started using RBD. A general description of the RBD software, including a discussion of the basic concepts underlying RBD and a description of the RBD menus, is in Sect. 4. Section 5 is the RBD tutorial - a set of ten lessons on using the RBD software package. This tutorial is followed (Sect. 6) by a description of the details of operation of the RBD programs and utilities. Section 7 is a description of the methods and algorithms that form the basis of RBD, and Sect. 8 is a technical description of the nuclide data files used by RBD. Finally, three appendices contain descriptions of the format for the input and output of the program that computes intake and committed dose (RBDCOMP), a fourth appendix contains a listing of the site-specific file for customizing the RBD software to your location, and a fifth appendix contains directions on using RBD in the EASY mode. 


\section{INSTALLATION OF RBD SOFTWARE PACKAGE}

\section{INSTALLATION DIRECTIONS}

The RBD software must reside in the RBD directory and in subdirectories under RBD. The structure of the subdirectories and their content are shown below:

root: $\quad \mathrm{RBD}$

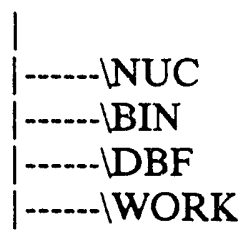

Batch (BAT) files

Nuclide data file (NUCLIDE.BIN)

Programs and utilities (EXE files)

Data base (DBF and NTX) files

Bioassay (DAT) and output (DBS,LOG,etc.) files

We assume either that you do not have a directory $\backslash R B D$ on the hard drive on which you wish to install RBD or that the directory is empty. Installation of RBD uses a free, copyrighted archive program to compress files on the distribution disks (LHARC Version $1.13 \mathrm{c}$ written by $\mathrm{H}$. Yoshizaki, 1989). The INSTALL procedure on the distribution disks will create the directory structure required for RBD and uncompress the files into the appropriate directory. The RBD software is distributed on four low-density 5-1/4-in. diskettes. To create RBD INSTALL diskettes for 3-1/2-in. or highdensity 5-1/4-in. diskettes, see the end of Sect. 2.2 on software package contents.

If you do not have a previous version of RBD, skip to the next page for instructions on installing RBD on your computer.

If you have a previous implementation of RBD with the RBD data base, please do the following:

- Back up the bioassay data in each of the RBD input data bases by (1) extracting the data from each data base and (2) copying all the RBD input files (DAT files) extracted to a floppy disk. To extract the input data from an RBD input data base, select RBD main menu Input Data Base, choose the input data base and extract ALL DATA. To copy the RBD input files (DAT files) to a floppy disk, use the main menu selection FILMAN. Prior to Ver. 4.0 of RBD one must change directory (CD) to the WORK subdirectory (or, in older versions of RBD, to the RBDBASE subdirectory) and copy all DAT files to a floppy disk. These files can be loaded into the new version of RBD using the AUTOLOAD feature.

- If using Ver. 4.0a of RBD, use main menu selection FLLMAN to copy all the RBD input and output data base files in the DBF subdirectory to a floppy disk.

If you have a previous implementation of RBD without the RBD data base, please do the following:

Copy all RBD DAT files from the RBD directory or the WORK subdirectory to a floppy disk. Editing of these files may be required if they are to be used with the present version of RBD.

Continue with the installation procedure described on the next page. 
Begin here if you do NOT have a previous version of RBD.

- You should make a backup copy of the RBD installation diskettes for safekeeping. The contents of the four low-density 5-1/4-in. diskettes can be copied to a single high-density 3-1/2-in. diskette. Install disks Nos. 1 and 2 can be copied to a high-density 5-1/4-in. diskette or a low-density 3-1/2-in. diskette. Install disks Nos. 3 and 4 can be copied to a high-density 5-1/4-in. diskette or a low-density 3-1/2-in. diskette. Any of these can be used to install the RBD software at a later date.

- At this point, check that the destination drive (C, D, etc.) has sufficient capacity for the RBD software, which requires at least 3.2 megabytes of disk space.

- Now begin installing the RBD software by placing RBD INSTALL DISK No. 1 in floppy drive A or B. Change to drive A or B and type INSTALL followed by destination drive letter. For example, type:

or

INSTALL C if installing on drive $C$,

INSTALL D if installing on drive $D$.

Note that you do not type a colon following the drive designation.

- You will be asked to insert INSTALL DISK No. 2 into the drive and to type INSTALL C or D. Likewise for INSTALL DISKS No. 3 and No. 4 for the low-density 5-1/4-in. install diskettes. There will be two INSTALL DISKS if RBD is supplied on high-density 5-1/4-in. or low-density 3-1/2-in. diskettes and only a single INSTALL DISK when RBD is supplied on a high-density 3-1/2-in. diskette.

- When installation of the RBD software package is complete, you will see the message

INSTALLATION OF RBD COMPLETED.

After installation of the RBD software package, you will see the RBD setup menu as shown on the following page. 
SETUPRBD

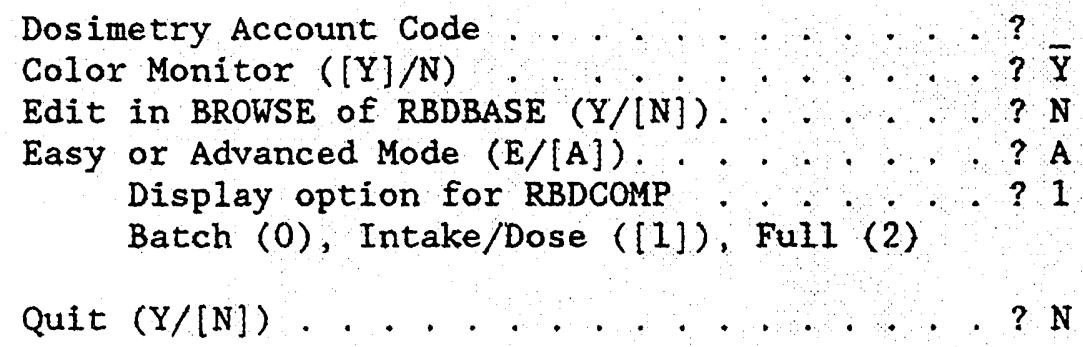

You need to enter the following information into the setup menu. Press <Enter $>$ after each response to go to the next item.

- You are first asked to specify a four-character Army dosimetry account code for your site. All RBD work files, including bioassay records files (DAT files), will have this dosimetry account code as the first part of the DAT filename. Entering a \$ for the account cole allows eight-character RBD input filenames. For the purposes of the tutorial that follows, enter DEMO as the dosimetry account code. The dosimetry account code is written to the file STATION.DFT in the DBF subdirectory. Later, after you have finished the sutorial, you may change the dosimetry account code by running SETUPRBD again by typing SETUPRBD at the DOS prompt in the RBD directory.

- You must specify whether or not you are using a color monitor. If you are using a color monitor attached to a color graphics adaptor (VGA, EGA, or CGA), respond Yes. Many computers have a color graphics adapter, but a monochrome monitor. If that is true in your case, respond No. The default response is Yes.

- Editing of RBD databases is normally restricted to the database utility EDITDBF. You can activate edit capability in the browse mode of RBDBASE by responding Yes to the next SETUPRBD query. If you do not wish to have edit capability in the browse mode respond No. The default response is $\mathrm{No}$.

- You must now specify whether to run RBD in the EASY or ADVANCED mode. The ADVANCED mode of RBD is described in the body of this report. The EASY mode is described in Appendix E. In the EASY mode, you are restricted to a single bioassay data base and a single output data base. In addition, you no longer need to select the input files for RBDCOMP or REPORT. To specify the EASY mode enter $E$. To specify the ADVANCED mode enter $A$, the default response. For purposes of the tutorial that follows, accept the default response, $A$, to select the ADVANCED mode. 
- If EASY mode is specified you will be asked to select the display option for RBDCOMP. The options available are: Batch (0), Intake/Dose (1), or Full (2). These options are described in Sect. 6. The same display option is used by the REPORT program. The default display option is 1 . If ADVANCED mode is specified, you do not specify the display option.

- Exit SETUPRBD by responding Yes to Quit?. You may change entries in the SETUP menu by responding No to Quit?. SETUPRBD leaves you in the RBD directory on completion. SETUPRBD may be run in the RBD directory at any time to change the setup parameters.

The installation and setup procedure is now complete. Remember to remove the install diskette from the floppy drive.

- Whenever you use RBD, change to the drive on which RBD is installed and CD to the RBD directory. Once there, type $R B D$ to get the RBD title screen. To use RBD you must always be in the RBD directory.

- Help for an RBD main menu item is available by pressing the F1 key when the menu bar is on the menu item. Help is available within the database programs (RBDBASE, RBDIMPOR, and EDITDBF) by pressing the F1 key. 
22 SOFTWARE PACKAGE CONTENTS (For four low-density, 5-1/4-in. diskettes)

This section contains a listing of the contents of the RBD installation diskettes. Archive files have extension LZH, and their contents can be listed using the archive facility LHARC provided on each diskette. To list the contents of all archive files, type: LHARC * . LZH.

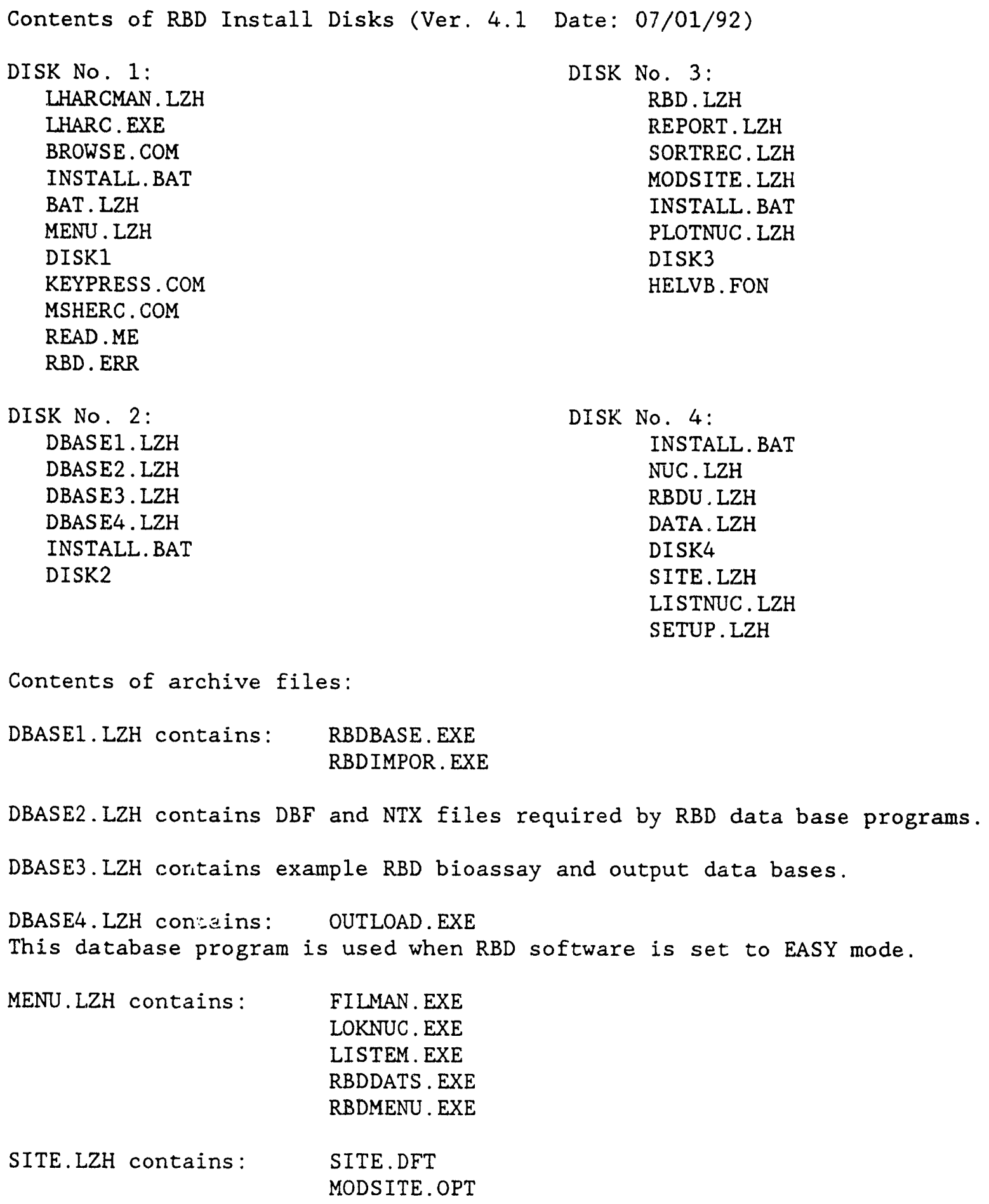

Contents of archive files:

DBASE1.LZH contains: RBDBASE. EXE RBDIMPOR. EXE

DBASE2. LZH contains DBF and NTX files required by RBD data base programs. DBASE3. LZH cortains example RBD bioassay and output data bases.

SITE.LZH contains: SITE.DFT MODSITE.OPT 


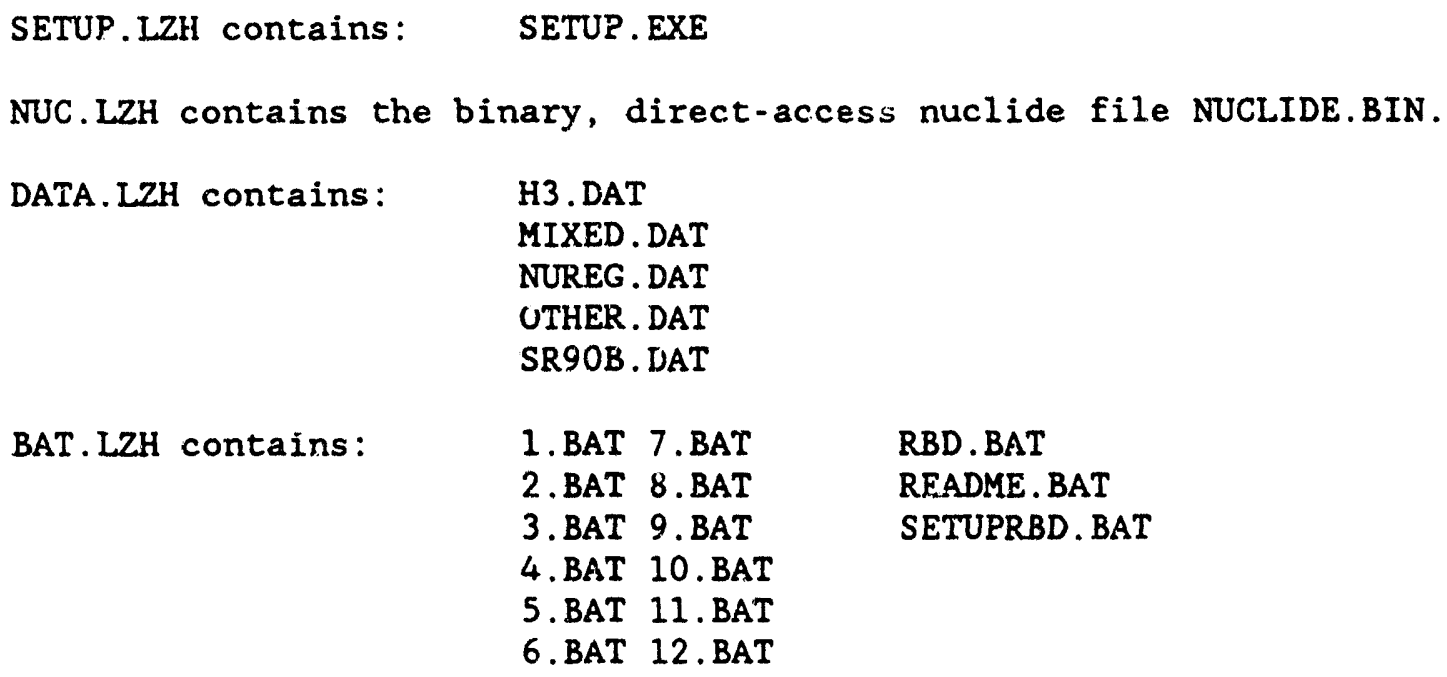

HELVB.FON is the MicroSoft FORTRAN font file used by the RBD plotting programs and utilities.

To create RBD INSTALL diskettes for low-density 3-1/2-in. or high-density 5-1/4-in. diskettes, copy the contents of INSTALL DISK No. 1 and INSTALL DISK No. 2 to one diskette and the contents of INSTALL DISK No. 3 and INSTALL DISK No. 4 to a second diskette. Install RBD sing the two diskettes in sequence.

To create RBD INSTALL diskettes using a single high-density 3-1/2-in. diskette, copy the contents of all four low-density 5-1/4-in. INSTALL diskettes to the high-density 3-1/2-in. diskette. RBD can then be installed using this single diskette.

\section{ERRORS AND ANOMALIES}

If you should encounter errors in running the RBD software package, please note carefully the context in which the error occurred and use the following RBD ERROR REPORT form to notify us of the problem. If the problem occurs in the bioassay program (RBDCOMP), it would be helpful if you could enclose a disk containing the bioassay records file (DAT file) that caused the problem. E-mail should be sent to rwd@ornl.gov. 
RBD ERROR REPORT

This RBD error report is provider: 25 the file RBD.ERR on the RBD INSTALL DISK No. 1.

The file can be edited and sent to the e-mail address given below.

SOFTWARE PACKAGE: RBD (Radiological Bioassay and Dosimetry)

RELEASE VERSION: 4.1 DATE: 07-01-92

RBD MAIN MENU ITEM IN WHICH ERROR OCCURRED:

DATE ERROR OCCURRED:

DESCRIPTION OF ERROR:

SUBMTTTED BY:

Name Date

Organization

Address

Phone

E-Mail

SEND TO:

Dr. Keith F. Eckerman

E-mail: rwd@ornl.gov

Bldg 7509, MS 6383

Oak Ridge National Laboratory

P. O. Box 2008

Oak Ridge, Tennessee $37831-6383$ 


\section{GETTING STARTED WITH RBD}

Before starting to use RBD, you should do the following:

- Check for the presence of a CONFIG.SYS file, which is usually found in the root directory of the $\mathrm{C}$ drive. The CONFIG.SYS file must have a line that sets FILES $=20$ or larger. It must also contain the line DEVIEE AMSI. SYS for proper scrolling of text screens within RBD. Reboot your system after modifying CONFIG.SYS.

- If the computer has a HERCULES graphics adapter, copy the file MSHERC.COM from RBD INSTALL DISK No. 1 to the root directory and type MSHERC. This puts the monitor in graphics mode and must be done every time the computer is rebooted (unless it is placed in the AUTOEXEC.BAT file) to take advantage of RBD's graphics capability when using a HERCULES graphics monitor.

- When starting RBD, you must always be in the RBD directory. If you abort out of any of the RBD programs by responding Yes to Terminate batch job $(\mathrm{Y} / \mathrm{N})$ ?, you may end up in a subdirectory of RBD. If this occurs, just $C D$ to the RBD directory.

Once in the RBD directory, type $R B D$ to get the RBD title screen. Press any key to get the RBD main menu, shown in Fig. 1.

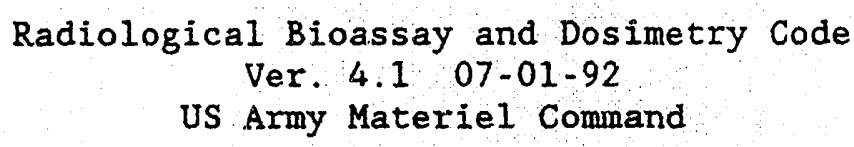

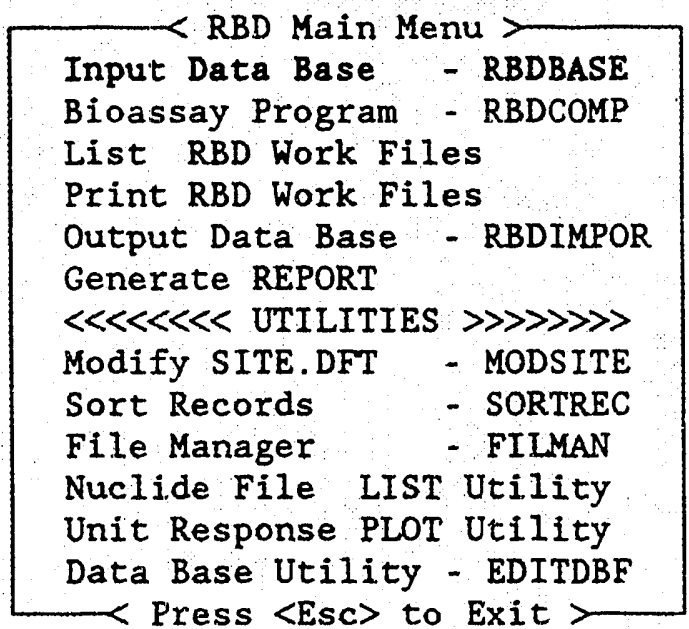

Use $\uparrow \downarrow$ to scroll menu, <Enter> to select, <FI> for help, <Esc> to Exit. Enter/Select/Sort/Extract Bioassay Records.

Fig. 1. RBD main menu. 
The RBD software is menu-driven. At the top level is the RBD main menu. To select a menu item, use the arrow keys to move the menu bar to that item and press the <Enter $>$ key. In most cases this will put you into an RBD submenu. To return to the main menu (top-level), press the escape $(<E s c>$ ) key. To exit the main menu, press the $<E s c>$ key.

- At any RBD submenu the <Esc > key will take you to the previous menu.

- Run SETUPRBD in the RBD directory to modify RBD setup parameters. The default values of the RBD setup parameters are shown below, including the Army dosimetry account code, assumed here to be DEMO. The setup parameters are described in Sect. 2. If you have a color monitor, the SETUPRBD menu should appear as follows prior to beginning the tutorial in Sect. 5.

SETUPRBD

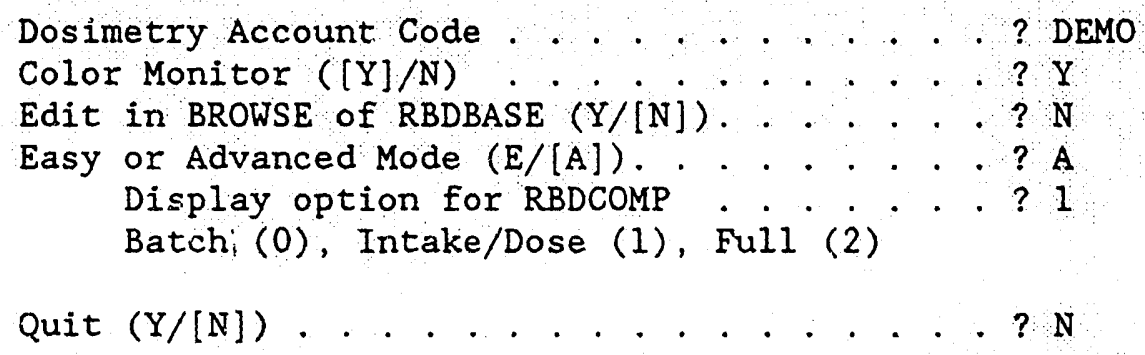

- Help for an RBD main menu item is available by pressing the F1 key when the menu bar is on the menu item. Help is available within the database programs (RBDBASE, RBDIMPOR, and EDITDBF) by pressing the F1 key. 


\section{DESCRIPTION OF RBD SOFTWARE PACKAGE}

The RBD software resides in the RBD directory and its subdirectories. The structure and content of the subdirectories is shown below:

root: $\quad$ RBD

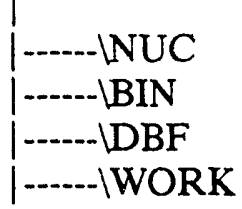

Batch (BAT) files

Nuclide data file (NUCLIDE.BIN)

Programs and utilities (EXE files)

Data base (DBF and NTX) files

Bioassay (DAT) and output (LOG,DBS,etc.) files

To use RBD, you must be in the RBD directory. Once there, type $R B D$ to get the RBD title screen. Press any key to get the RBD main menu, shown below.
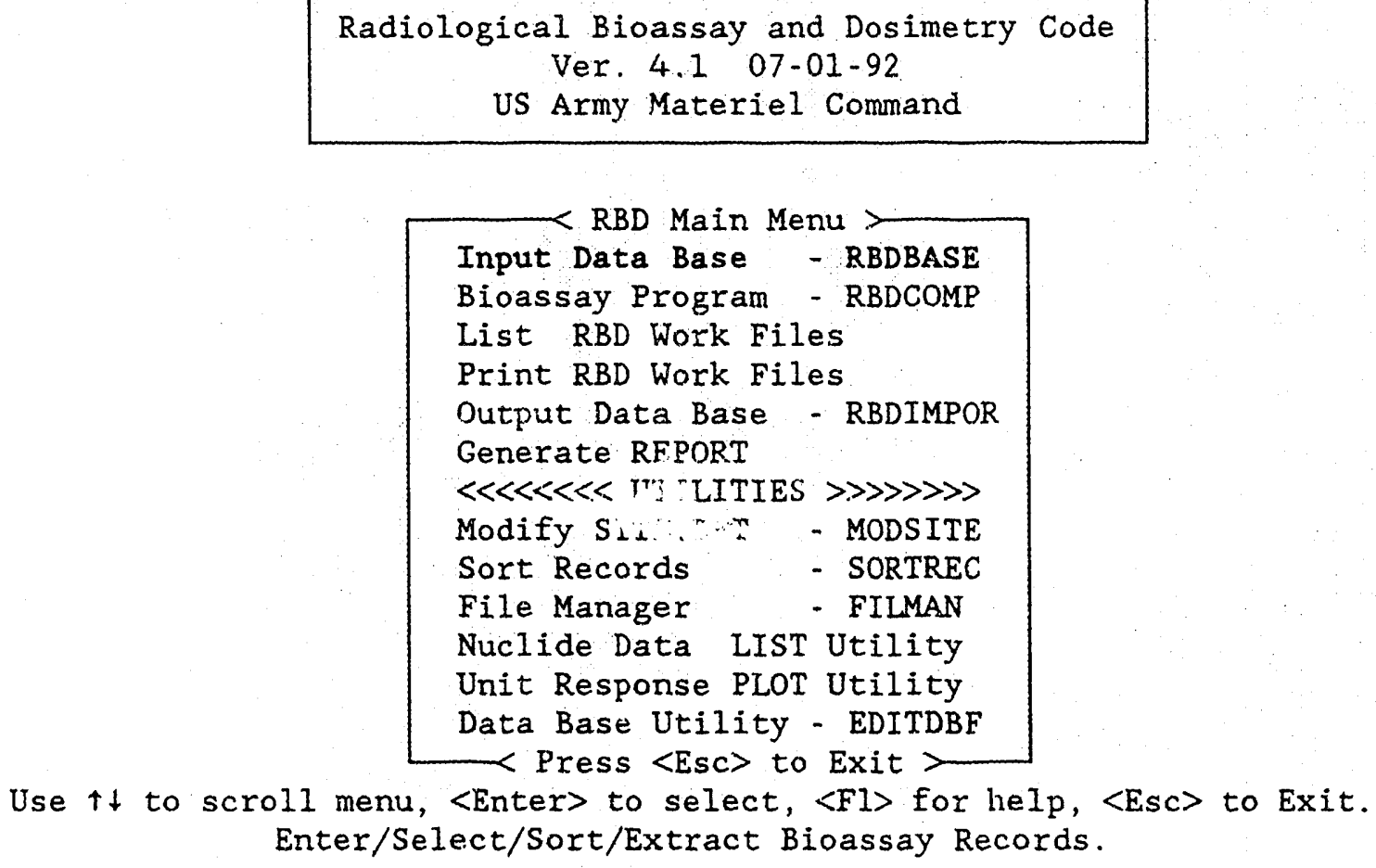

\section{RBD Main Menu}

The RBD software is menu-driven. At the top level is the RBD main menu shown above. To select a menu item, move the menu bar to that item and press <Enter $>$. You can also type the first letter of a menu item to select that item. You can return to the main menu, from anywhere in the RBD software, by pressing a series of escape keys (<Esc $>$ ). To return to the DOS prompt from the RBD main menu, press $<$ Esc $>$. 
RRD consists of four programs plus several utilities. The four RBD programs are the bioassay data $t$ use program (RBDBASE), the bioassay program (RBDCOMP), the REPORT program, and the output data base program (RBDIMPOR). The two data base programs, RBDBASE and RBDIMPOR, manage the bioassay data base and output data base, respectively. The bioassay program (RBDCOMP) computes intake and committed dose. The REPORT program computes weighted intake and committed dose measurements from multiple bioassays for exposure to a given nuclide and class.

The iullowing gives an overview of data flow through RBD and the file-naming conventions used by the RBD software. A data flowchart is shown in Fig. 2 . In the flow chart, RBD programs are shown in bold, and data bases and records files are shown in italics.

- Bioassay data base names begin with RBD, and output data base names begin with OUT. Both kinds of data bases have extension DBF and are located in subdirectory DBF. Every data base has an index file (with extension NTX) also located in subdirectory DBF.

- Input for the bioassay program (RBDCOMP) is extracted from the bioassay data base by the bioassay data base program (RBDBASE) into files called bioassay records files. These files have the extension DAT and are sometimes referred to as DAT files.

- Output from the bioassay program is written into two files - the output records file (with extension DBS) and the output listing file (with extension LOG). These files are sometimes referred to as DBS and LOG files.

- The output records in DBS files are imported into an output data base with the output data base program (RBDIMPOR).

- The REPORT program produces a committed effective dose (CED) file from the DBS file using weighting factors for the results of multiple bioassays. The CED file becomes input for the U.S. Army dosimetry data base located at Huntsville, Alabama.

- The REPORT program also produces two output listing files: the complete listing (with extension REP) and a summary listing (with extension SUM).

- The DAT, DBS, LOG, CED, REP, and SUM files are referred to as RBD work files. 

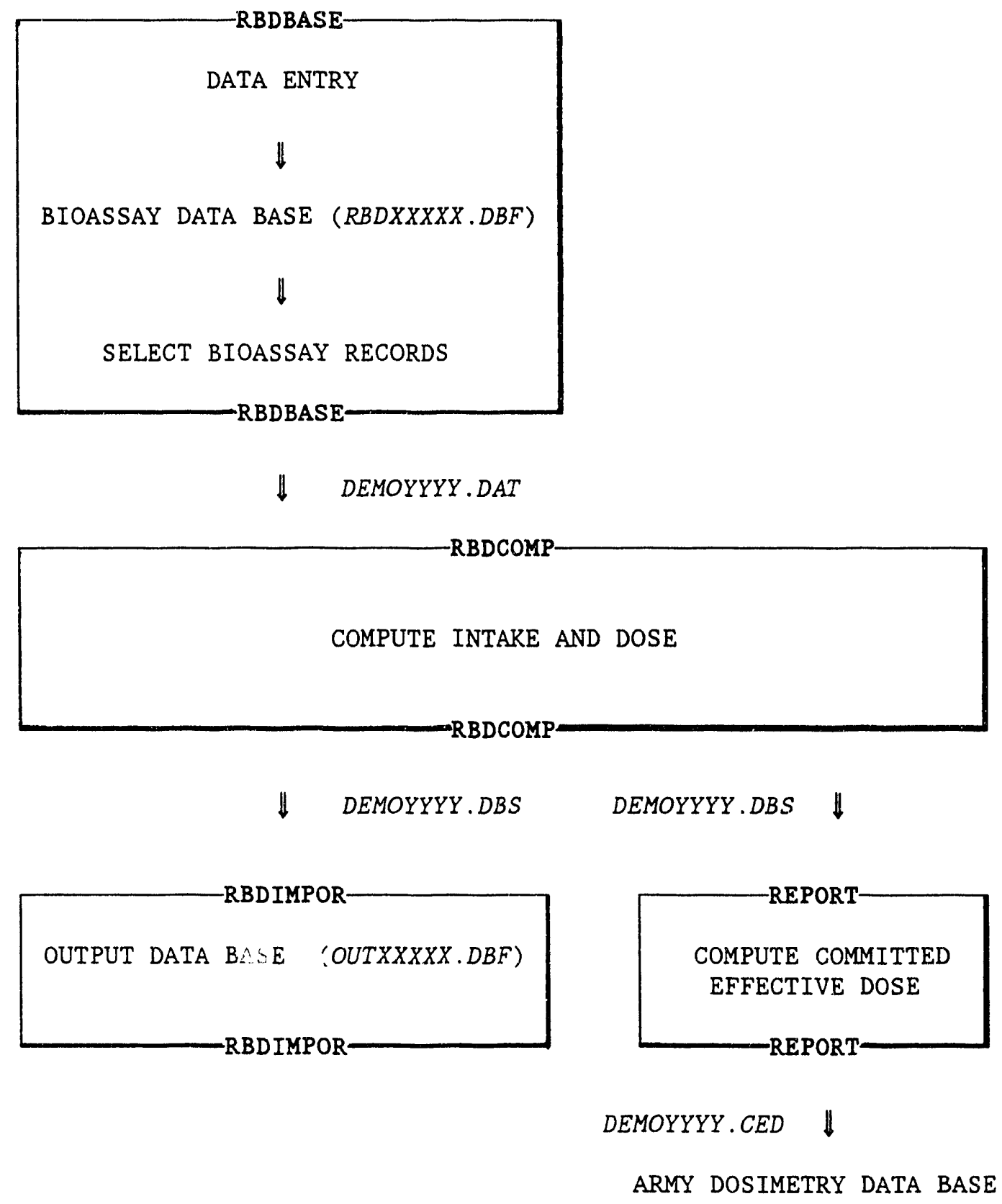

Fig. 2. Data flow through the RBD software package assuming that the dosimetry account code is DEMO. RBD programs are shown in bold, and data bases and records files are shown in italics. The portions of the data base names noted as $X X X X X$ and records file names noted as $Y Y Y Y$ are supplied by the user in the ADVANCED mode of operation. 


\subsection{USE OF RBD WITHIN THE ARMY DOSIMETRY PROGRAM}

The RBD software was developed for the U.S. Army to be used both at a field site and a central location. At the field site, bioassay data would be entered into the RBD data base, bioassay records extracted, and the intake and committed dose computed. Bioassay and output data bases would store the bioassay records and the output records (estimates of intake and committed dose) for individuals at the site. The field site personnel would send two files (DAT and DBS files) to the central location, where another copy of the RBD software (residing on a mainframe computer) would recompute the intake and committed dose for all monitored U.S. Army personnel. The final results for committed effective dose equivalent for all Army personnel monitored (the CED file) would be combined with the dose from external exposure. The central location would archive the input and output to the RBD bioassay program, the DAT and DBS files, respectively.

\subsection{BASIC CONCEPTS UNDERLYING THE RBD SOFTWARE}

Monitoring of individuals for internal exposure is based on the measurement of radionuclides in the whole body (or specific organs) and on the measurement of radionuclides in excreta. RBD computes the intake and committed dose using these measurements, along with biokinetic and dosimetric models describing the behavior of a unit intake of the material in Reference Man. Certain fundamental concepts must be defined in developing an internal dosimetry package such as RBD. เn this section we define and discuss those concepts.

\section{PATHWAY}

The route of intake of the radionuclide into the body of the individual is referred to as the PATHWAY. RBD allows three different routes of intake: inhalation, ingestion, and wound. Table 1 summarizes the codes for the bioassay records, including the codes for pathway.

\section{NUCLIDE}

The RBD software computes intake and committed dose for a specified radionuclide. This is often referred to as the NUCLDE. Within the RBD software and documentation, nuclides are referred to using the notation I-131 for ${ }^{131} \mathrm{I}$, for example. RBD can compute intake and committed dose only for nuclides for which there exists a nuclide data file. At present there are 41 nuclide data files:

$\begin{array}{lllllllll}\text { H-3 } & \text { C-11 } & \text { C-14 } & \text { Na-22 } & \text { Na-24 } & \text { P-32 } & \text { Co-57 } & \text { Co-58 } & \text { Co-60 } \\ \text { Ni-63 } & \text { Sr-85 } & \text { Sr-89 } & \text { Sr-90 } & \text { Tc-99 } & \text { Tc-99M } & \text { I-125 } & \text { I-129 } & \text { I-131 } \\ \text { I-135 } & \text { Cs-134 } & \text { Cs-137 } & \text { Pm-147 } & \text { Eu-152 } & \text { Eu-154 } & \text { Eu-155 } & \text { Tl-201 } & \text { Ra-244 } \\ \text { Ra-226 } & \text { Th-228 } & \text { Th-232 } & \text { U-234 } & \text { U-235 } & \text { U-236 } & \text { U-238 } & \text { U-NAT } & \text { Pu-238 } \\ \text { Pu-239 } & \text { Pu-241 } & \text { Am-242 } & \text { Cm-242 } & \text { Cm-244 } & & & & \end{array}$


Table 1: Codes for bioassay records.

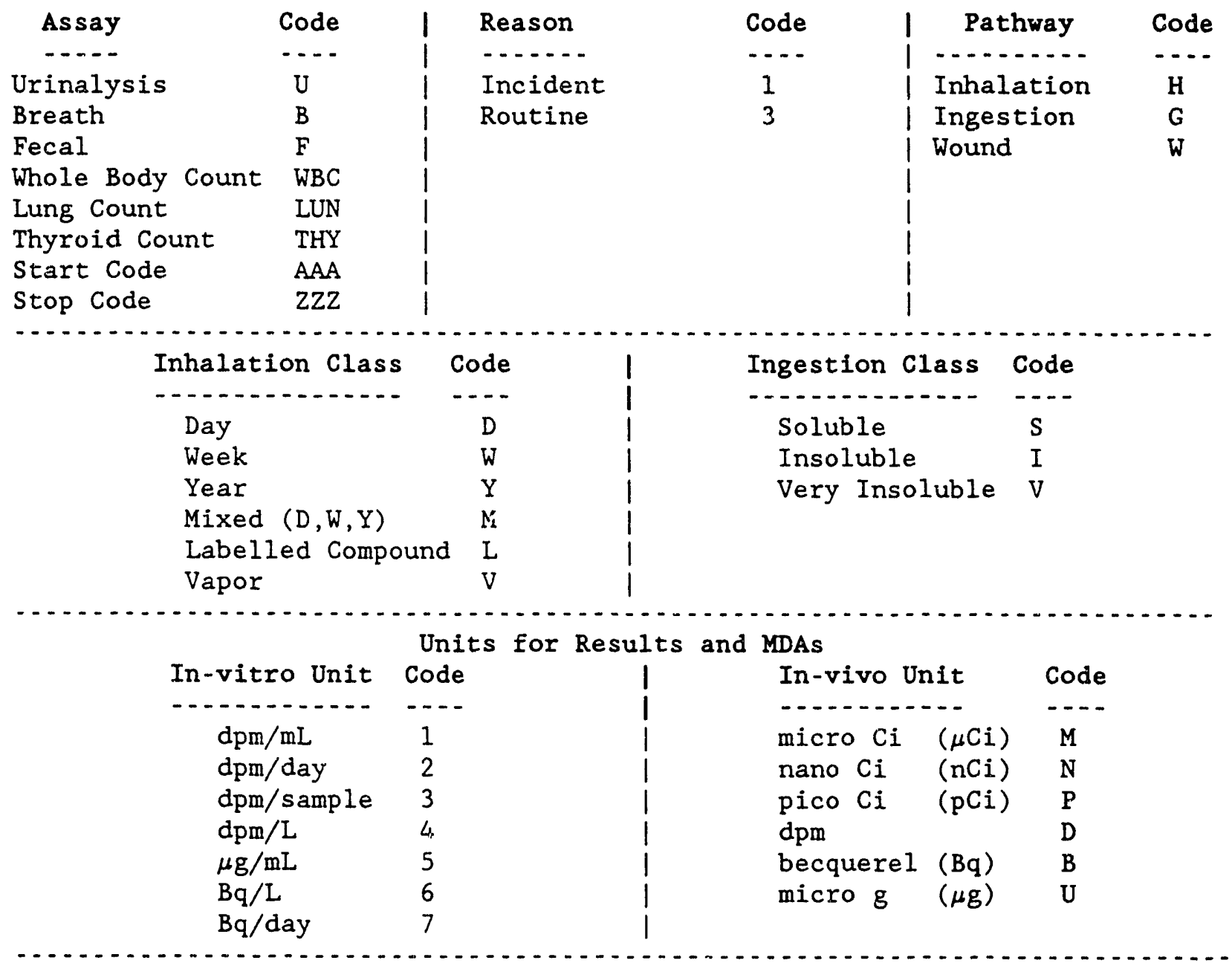




\title{
CLASS
}

Radionuclides have been classified according to their rate of clearance from the pulmonary region (for inhaled radionuclides) or the fraction of material reaching body fluids following ingestion (for ingested radionuclides). The former is referred to as the inhalation class of the nuclide, and the latter is referred to as the $f_{1}$ parameter, which we translate into solubility codes. Solubility codes of $S, I$, and $\mathrm{V}$ refer to soluble, insoluble, and very insoluble forms of the ingested material, respectively, corresponding to decreasing numerical values of $\mathrm{f}_{1}$. The term "mixed inhalation class" or MTXED CLASS refers to a material that has clearance properties which are described as a mixture, by percentage, of the standard inhalation classes: days (D), weeks (W), and years (Y). Table 1 summarizes the aliowed radionuclide classes and their corresponding bioassay records codes.

\section{REASON}

The type of computation performed by the RBD bioassay program (RBDCOMP) is determined by whether the individual's exposure to the radionuclide was acute or chronic. In the RBD bioassay program and documentation, the term INCIDENT is used synonymously with the term "acute," and the term ROUTINE is used synonymously with "chronic." You must supply the proper REASON code for the computation of intake and committed dose for the RBD software to work properly. Table 1 summarizes the allowed values of REASON and their corresponding bioassay records codes.

\section{INCIDENT DATE and TTME}

For acute (INCIDENT) exposure you should specify an INCIDENT DATE and an INCIDENT TIME. These data are used to uniquely define each incident. If the incident date and time are missing, the RBDCOMP program assumes a value of $\mathrm{N}$ days prior to the date of the first bioassay measurement, where $\mathrm{N}$ is specified in the site-specific data file (see Sect. 6).

\section{CASE}

Each example of exposure of an individual to a particular radionuclide (NUCLIDE) of a particular inhalation or ingestion class (CLASS) for a given situation (REASON), be it an incident exposure or routine exposure, is referred to as a CASE A CASE is limited to a maximum of 200 bioassay records.

\begin{abstract}
ASSAY
The RBD software computes intake and committed dose for the bioassay measurements provided for each case. Bioassay measurements, often referred to generally as ASSAYS, can be either in vivo or in vitro. In vivo measurements allowed in RBD are whole body count (WBC), lung count (LUN), and thyroid count (THY). In vitro assays allowed in RBD are urinalysis (U), fecal analysis (F), and breath analysis (BRE). Table 1 shows MEASUREMENT UNITS used for in vivo and in vitro measurements. Integer codes are used for in vitro assay, and letter codes are used for in vivo measurements. You are prompted in the RBD bioassay data entry form as to which units are allowed for a selected assay.
\end{abstract}


MDA

The MDA, or minimum detectable activity, is the minimum amount of the nuclide detectable by a given assay method. The MDA can and should be specified on each bioassay record. The RBD software uses all measurement values to compute intake and committed dose, even if those values are negative. RBDCOMP will indicate that an intake is confirmed only if there are two or more measurements above the lower critical limit, which is defined in the site-specific file to be half of the MDA value. The MDA for each measurement can have the same or different units from the measurement itself, but is restricted to the same set of units as defined for the corresponding assay. This is indicated in the bioassay data entry form.

\section{EXPOSURE HISTORY}

Special codes (in place of assay codes) are used to signify start and eno of chronic exposure of an individual to a given nuclide and class of nuclide. The start code is specified by AAA in the assay field and the end code by $\mathrm{ZZZ}$ in the assay field. You insert exposure history records by selecting the exposure history menu item from the bioassay data base menu and entering the date (and, optionally, time) when the individual either started (START) or ended (END) exposure to the specified nuclide and class of nuclide, along with the corresponding code (AAA or ZZZ).

For RBD to function correctly you must provide the NUCLIDE, CLASS, PATHWAY, and REASON on each bioassay record. If REASON is specified as INCIDENT, then you should provide the INCIDENT DATE and, optionally, the INCIDENT TIME

\section{MEASUREMENT WINDOW}

Two global time windows are used by the RBD software. These windows are entered after the bioassay records files have been selected and the RBD bioassay program has started. If routine measurements are present, you will be asked for a starting date, in the form YYYY, and a duration (in years) for considering measurements for routine analysis. This period is referred to as the MEASUREMENT WINDOW. The measurement window brackets the period of time during which routine measurements will be considered for computing intake rates. You are not asked for the MEASUREMENT WINDOW unless routine records must be processed. Note that this window will be applied to $2 ?$ chronic exposure cases by RBDCOMP unless this information is superseded by an exposure history record. 


\section{INTAKE WINDOW}

You are then asked for a starting date, in the form YYYY, and a duration (in years) for computing the intake. This period is referred to as the INTAKE WINDOW. The intake window is the period of time over which the intake for routine assay is evaluated. Assays associated with an incident will be evaluted only if the incident date falls within the intake window.

The measurement and intake windows are illustrated in Fig. 3. Consider first a sequence of routine bioassay measurements for this individual. These values are shown by downward-pointing arrows at the top of the figure. For an analysis of radiation exposure in the calendar year 1990, the MEASUREMENT WINDOW was taken to extend over the years 1989 and 1990 . This is shown by the horizontal arrow in the middle of the figure. The measurements actually considered for determining the intake are shown below the measurement window. Note that measurements prior to 1989 are not considered. Below the measurement window is a second horizontal arrow that indicates the INTAKE WINDOW, the period over which the intake is computed for the routine measurements. Note that the measurements in the year 1989 are used to determine the intake rate at the beginning of the year 1990 and, therefore, will affect the computation of the intake in the year 1990. It is not necessary that the measurement and intake windows coincide with the beginning and ending of the year.

Now consider a set of acute measurements in the year 1990 as shown by upward-pointing arrows at the bottom of the figure. For RBD to compute intake for this incident, the incident date must fall within the intake window, which it does.

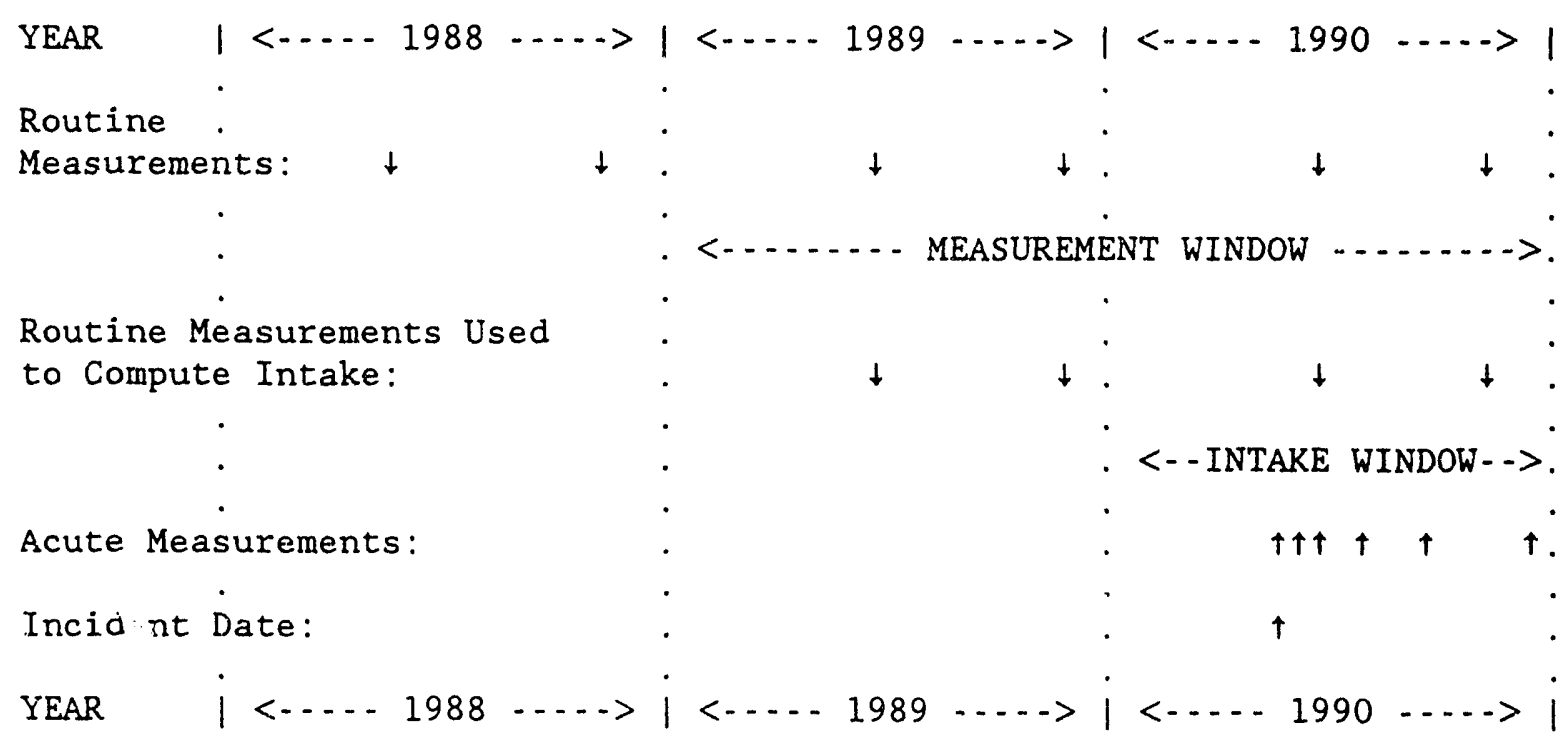

Fig. 3. Illustration of the measurement and intake windows. 


\section{NUCLIDE DATA FLLS}

For each radionuclide there exists a Nuclide Data File that contains information that the RBD bioassay program requires to carry out the calculation of intake and committed dose for that nuclide. The file contains the nuclide mass, radioactive half-life, transfer rates, excretion and retention functions, dose per unit deposition for inhaled activity, and dose per unit intake for ingested activity. The nuclide data files were designed to minimize the necessity for modifying the RBD bioassay program when changes are made to the lung model, GI-tract model, and to excretion and retention functions. The individual nuclide data files are combined into a single binary, direct-access file (NUCLIDE.BIN) to speed data access.

\section{SITE.DFT - THE SITE-SPECIFIC FILE}

The site-specific file, SITE.DFT, is used by the RBD software. It contains default values of bioassay records parameters to be used if these parameters are missing. In addition, the SITE.DFT file contains parameters that control the operation of RBDCOMP and REPORT. Lesson 1 illustrates how to modify the site-specific file.

\section{RBDBASE PROGRAM}

Bioassay data bases are accessed using the program RBDBASE. RBDBASE was developed using the Clipper data base language, which is similar to DBASE III+. This program gives the RBD user the capability to enter bioassay data directly into ? selected or newly created bioassay data base or, with the AUTOLOAD utility, to load existing bioassay records files (DAT files) into a bioassay data base. In addition, the program allows you to browse the data base and to extract bioassay records into DAT files for input to the bioassay program (RBDCOMP).

\section{BIOASSAY PROGRAM}

The bioassay program (RBDCOMP) is the part of the RBD software that computes the intake and committed dose when given a set of bioassay records. This code is written in FORTRAN-77 and uses MicroSoft's FORTRAN graphics calls to display plots on the screen. To get hard copy of the displayed plots, you must use a graphics screen dump utility. If you have DOS 5.0, VGA graphics screen dumps can be obtained by loading the DOS routine GRAPHICS (with the $/ R$ option) prior to running RBDCOMP. The /R option means to print the screen as is with colors converted to halftones using the DOS file GRAPHICS.PRO. The colors used by the plotting routines in RBD can be modified in the site-specific file (SITE.DFT) to obtain the best printed output. Some screen dump utilities make certain assumptions (e.g., yellow is a background color) that cause strange results in the printed output.

\section{REPORT PROGRAM}

The REPORT program is used to prepare a committed dose report that shows the committed effective dose to each individual. The REPORT program reads the RBDCOMP output records file (DBS file). When measurements from multiple bioassays are present for an exposure to a given nuclide and class, REPORT estimates the intake and dose by applying weights assigned in the nuclide data file for each assay. Two output listings are created by the REPORT program: the complete listing (with extension REP) and a summary listing (with extension SUM). The committed effective 
dose is written to a file with the extension CED.

\section{RBDIMPOR PROGRAM}

Output data bases are accessed using the program RBDIMPOR. RBDIMPOR was developed using the Clipper data base language, which is similar to DBASE III+. This program allows you to load output records files (DBS files) into the data base, browse the output data base, and to determine which bioassay data were used to compute corresponding output.

\section{BROWSE UTILITY}

The utility BROWSE is used, by various sections of the RBD software, to examine files. To exit BROWSE, press <ESC $>$. 


\section{DESCRIPTION OF RBD MAN MENU ITEMS}

This section addresses each item on the RBD main menu assuming RBD is operating in the ADVANCED mode. The purpose of the item is briefly cescribed, and, in general, the next menu that appears after selecting the main menu item is shown. The sequence of the menu items largely corresponds to the flow of data shown in Fig. 2.

\section{Input Data Base - RBDBASE}

This menu item is used to create a new bioassay data base or to select an existing bioassay data base for the purpose of adding records. RBDBASE is also used to select bioassay records from the data base and extract them into a DAT file for computation of intake and committed dose. An empty output data base is automatically created when a bioassay (input) data base is created.

The data base files have extension $\mathrm{DBF}$. The first three characters of a bioassay data base are RBD and the first three characters of the corresponding output data base are OUT. Due to this convention and because each data base has an associated index file, you should not modify the name of a biosssay or an output data base file.

Below is the RBDBASE menu, which appears after you select a bioassay data base.

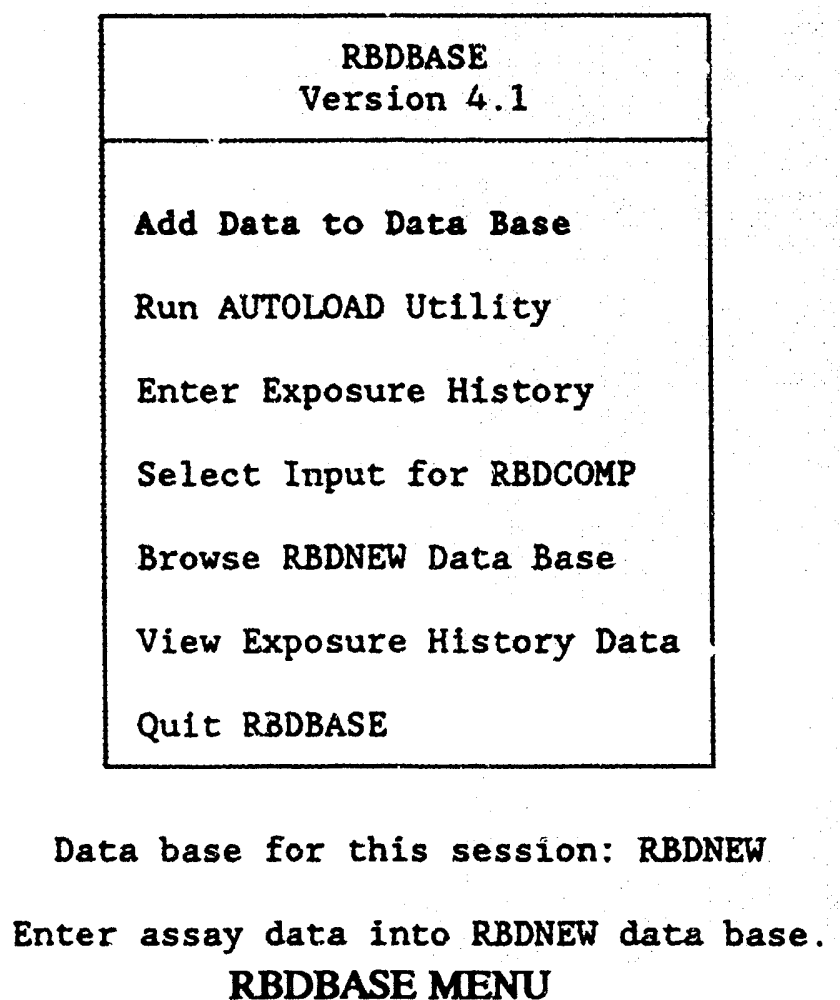

RBDBASE MENU 


\section{Bioassay Program - RBDCOMP}

This menu item is used to execute the bioassay program using a set of bioassay records (a DAT file) previously selected using RBDBASE. The measurements per unit intake and the expected biological response per unit intake will be plotted if the PLOT switch in SITE.DFT is set to true (T). A committed dose report is automatically generated after an RBDCOMP run if the REPORT switch in SITE.DFT is set to true (T).

Below is the menu for selecting input files for the bioassay program.

$$
\begin{gathered}
\text { Radiological Bioassay and Dosimetry Code } \\
\text { RBDCOMP Ver. } 4.1 \text { 07-01-92 }
\end{gathered}
$$

\begin{tabular}{llrr}
\hline Select Files for & RBDCOMP Input \\
1. H3.DAT & 6729 & $6-07-91$ & $4: 41 \mathrm{p}$ \\
2. MIXED.DAT & 1624 & $10-28-91$ & $3: 28 \mathrm{p}$ \\
3. NUREG.DAT & 3480 & $10-28-91$ & $3: 24 \mathrm{p}$ \\
4. OTHER.DAT & 8816 & $10-28-91$ & $3: 24 \mathrm{a}$ \\
5. SR9OB.DAT & 1625 & $6-11-91$ & $12: 31 \mathrm{a}$ \\
-.................. & & \\
Execute RBDCOMP Program & & \\
Quit to Menu
\end{tabular}

To select, move menu bar to item and press <Enter>, or type number. To cancel, repeat above. After selections are made, press E to execute. 


\section{Output Data Base - RBDLMPOR}

This menu item is used to select an existing output data base for loading the results from the RBDCOMP program (the DBS file) into this output data base. The user can also browse the output data base and determine which bioassay records produced a given result.

Below is the RBDIMPOR menu, which appears after you select an output data base. The output data base selected for this example was OUTNUREG.

\begin{tabular}{l} 
RBDIMPOR Ver. 4.1 \\
RBDCOMP Output File $->$ Output Data Base \\
\hline Import DBS FIle Into OUTNUREG Data Base \\
BROWSE OUTNUREG by Calculation Date \\
BROWSE OUTNUREG by SSN \& Nuclide \\
Show RBDNUREG Data That Produced Given Result \\
Quit RBDIMPOR
\end{tabular}

Data bases for this session are OUTNUREG and RBDNUREG.

Import DBS file from RBDCOMP into OUTNUREG output data base.

RBDIMPOR MENU 


\section{Generate REPORT}

This menu item is used to generate a REPORT from an existing output records file (DBS file). Two output listings are created by the REPORT program: (1) the complete listing, with extension REP and (2) a summary listing, with extension SUM. The committed effective dose is written to a file with the extension CED. Below is the menu for selecting input files for the REPORT program.

$$
\begin{aligned}
& \text { Radiological Bioassay and Dosimetry Code } \\
& \text { REPORT Ver 4.1 } 07-01-92 \\
& \text { Select Files for REPORT Input } \\
& \text { 1. H3.DBS } \quad 315 \quad 6-12-92 \quad 4: 58 \mathrm{p} \\
& \text { 2. MIXED.DBS } \quad 315 \quad 6-12-92 \quad 4: 58 \mathrm{p} \\
& \text { 3. NUREG.DBS } 1575 \quad 6-12-92 \quad 4: 58 \mathrm{p} \\
& \text { 4. OTHER.DBS } 1260 \quad 6-12-92 \quad 4: 59 \mathrm{p} \\
& \text { 5. SR9OB.DBS } 315 \quad 6-12-92 \quad 4: 59 \mathrm{p} \\
& \text { - - - - . - - - - - } \\
& \text { Execute REPORT Program } \\
& \text { Quit to Menu }
\end{aligned}
$$

To select, move menu bar to item and press <Enter>, or type number. To cancel, repeat above. After selections are made, press E to execute. 


\section{Modify SITEDFT - MODSITE}

This menu item (MODSITE utility) is used to modify the SITE.DFT file, which controls the computation of intake and dose, the plotting of excretion/retention functions, and the generation of the committed dose report.

Below is the MODSITE utility menu.

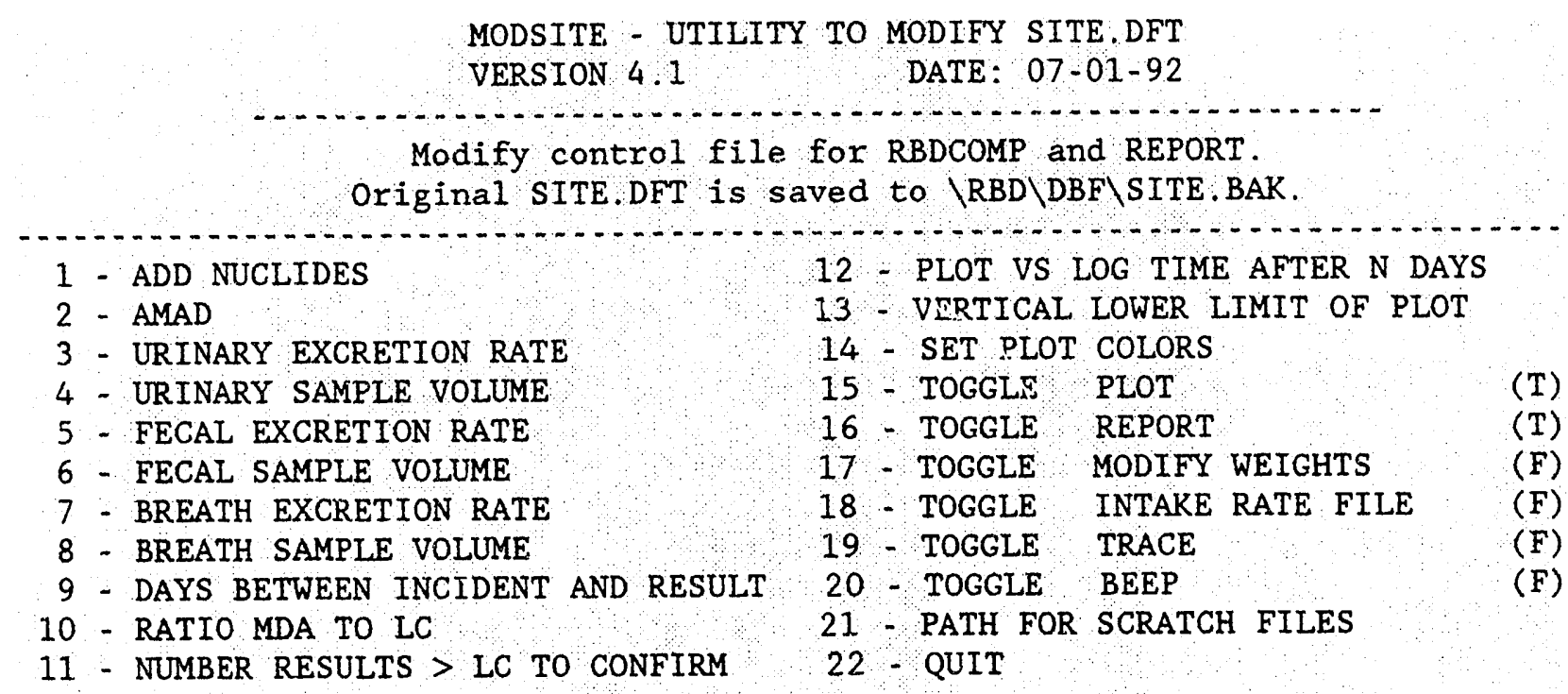

ENTER SELECTION: 
Sort Records - SORTREC

This menu item (SORTREC utility) is used to sort bioassay records, if several bioassay records (DAT) files have been combined. It can also be used to sort output records (DBS) files and provide statistics on the number of records, number of individuals, and nuclides occurring in the combined and sorted DAT and DBS files. This utility will also indicate when the assay weighting factors in the DBS file have been altered. SORTREC can sort 7000 records.

Below is the SORTREC utility menu.

$$
\begin{aligned}
& \text { Radiological Bioassay and Dosimetry Code } \\
& \text { SORTREC Ver. 4.1 07-01-92 }
\end{aligned}
$$

1. H3.DAT 6729 H3.DBS 315

To select, move menu bar to item and press <Enter>, or type number. To cancel, repeat above. After selections are made, press E to execute. 
File Manager - FULMAN

This menu item (FILMAN utility) is used to archive (copy) bioassay and output data bases to a floppy disk in selected drive (A or B ) or retrieve bioassay and output data bases from a floppy disk in selected drive (A or B). The FILMAN utility can also be used to archive (copy) RBD work files (DAT, DBS, CED, LOG, REP, and SUM files) to a floppy disk in selected drive (A or B ) or retrieve $\mathrm{RBD}$ work files from a floppy disk in selected drive (A or $\mathrm{B}$ ).

Below is the FILMAN utility menu.

Radiological Bioassay and Dosimetry Code

FILMAN Ver. 4.1 07-01-92

Select Task
Download data base files
Upload data base files
Download RBD work files
Upload RBD work files
Quit to Menu




\section{Nuclide Data LIST Utility}

This menu item is used to list the contents of a nuclide file. You can create a listing of the contents of a nuclide file (with extension LST), which can be browsed using LIST menu item or printed using PRINT menu item.

Below is the Nuclide Data LIST Utility menu obtained after selecting Co-60 as the nuclide.

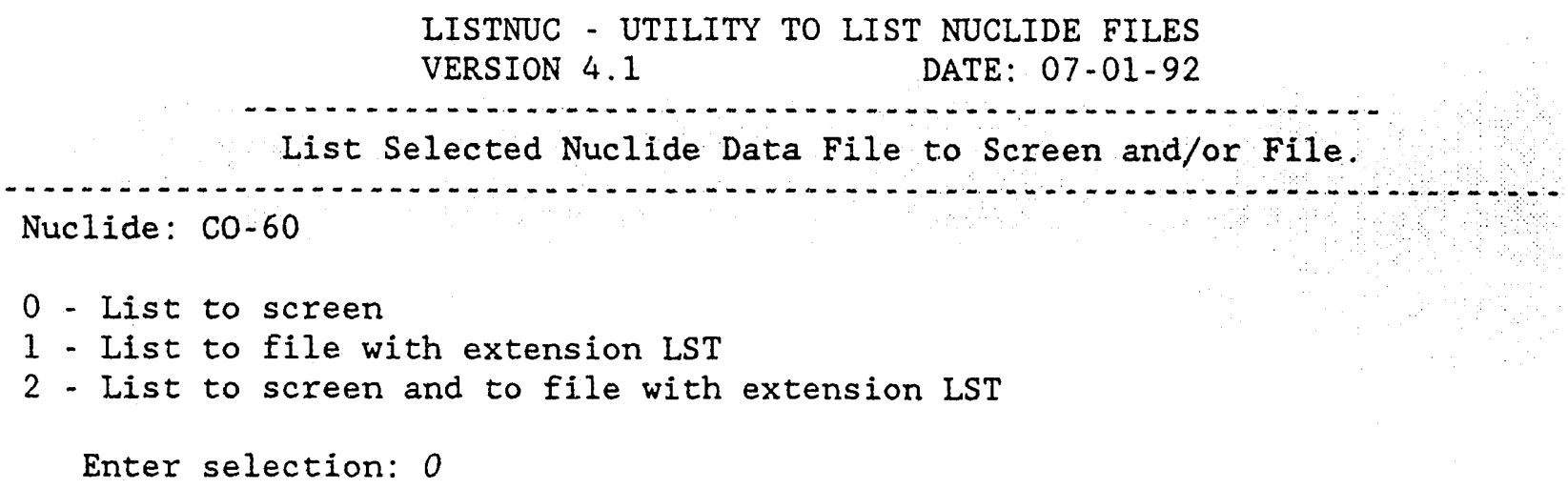

\section{Unit Response PLOT Utility}

This menu item is used to plot the intake retention or excretion function for a nuclide. You can also create a file (with extension PLT) tabulating the functions versus time.

Below is the Unit Response PLOT Utility menu obtained after selecting Co-60 as the nuclide.

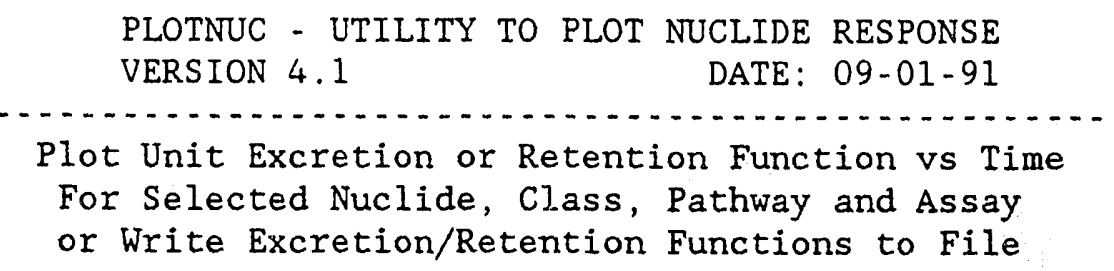

Nuclide: $60-60$

0 - Plot a retention/excretion curve

1 - Write retention and excretion plot data to file with extension PLT

Enter selection: 0 


\section{Data Base Utility - EDITDBF}

This menu item invokes a data base utility, EDITDBF, to edit or zap bioassay and output data base (DBF) files. The zap function will delete all records in the data base. Below is the EDITDBF menu.

\begin{tabular}{|l|}
\hline EDITDBF Utility \\
Browse/Edit RBDTEST data base \\
Zap the RBDTEST data base \\
Browse/Edit OUTTEST data base \\
Zap the oUTTEST data base \\
Select another data base \\
Quit and return to RBD main menu
\end{tabular}

RBDTEST contains 30 records. OUTTEST contains 4 records. Examine and edit bioassay records in RBDTEST data base. 


\section{RBD SOFTWARE TUTORIAL}

\section{CONTENTS}

The following lessons are contained in the RBD Software Tutorial:

Lesson 1. Modifying the site-specific file SITE.DFT

Lesson 2. Entering bioassay data into a bioassay data base.

Lesson 3. Browsing records in a bioassay data base.

Lesson 4. Extracting records from a bioassay data base and computing intake and dose using RBDCOMP.

Lesson 5. Importing records into a bioassay data base using AUTOLOAD.

Lesson 6. Importing RBDCOMP output into an output data base.

Lesson 7. Browsing records in an output data base.

Lesson 8. Entering exposure history data into a bioassay data base, extracting records, and computing intake and dose.

Lesson 9. Using the measurement and intake windows in computing intake and dose.

Lesson 10. Generating a committed dose report using REPORT.

All lessons assume you are at the RBD main menu. To obtain the RBD main menu, change to the RBD directory and type $R B D$. Run SETUPRBD to ensure that RBD is in the ADVANCED mode of operation.

In the discussion that follows, tutorial directives are surrounded by boxes such as this. User's response to prompts are shown in italics, and the menu item highlighted by the menu bar appears in bold. Bioassay and output data bases are denoted in italics. Bioassay records (DAT) files and output records (DBS) files are also denoted in italics. In this tutorial, alphabetic and numeric characters will appear in the social security field, which is normally a nine-digit number. 


\subsection{LESSON 1. MODIFYING THE SITE-SPECIFIC FILE SITEDFT}

In the first lesson you will learn how to modify the site-specific file, SITE.DFT, which controls operation of the RBD software. The MODSITE utility is used to make modifications to the SITE.DFT file.

Select Modify SITE.DFT - MODSITE from the RBD main menu.

You will see the following menu:

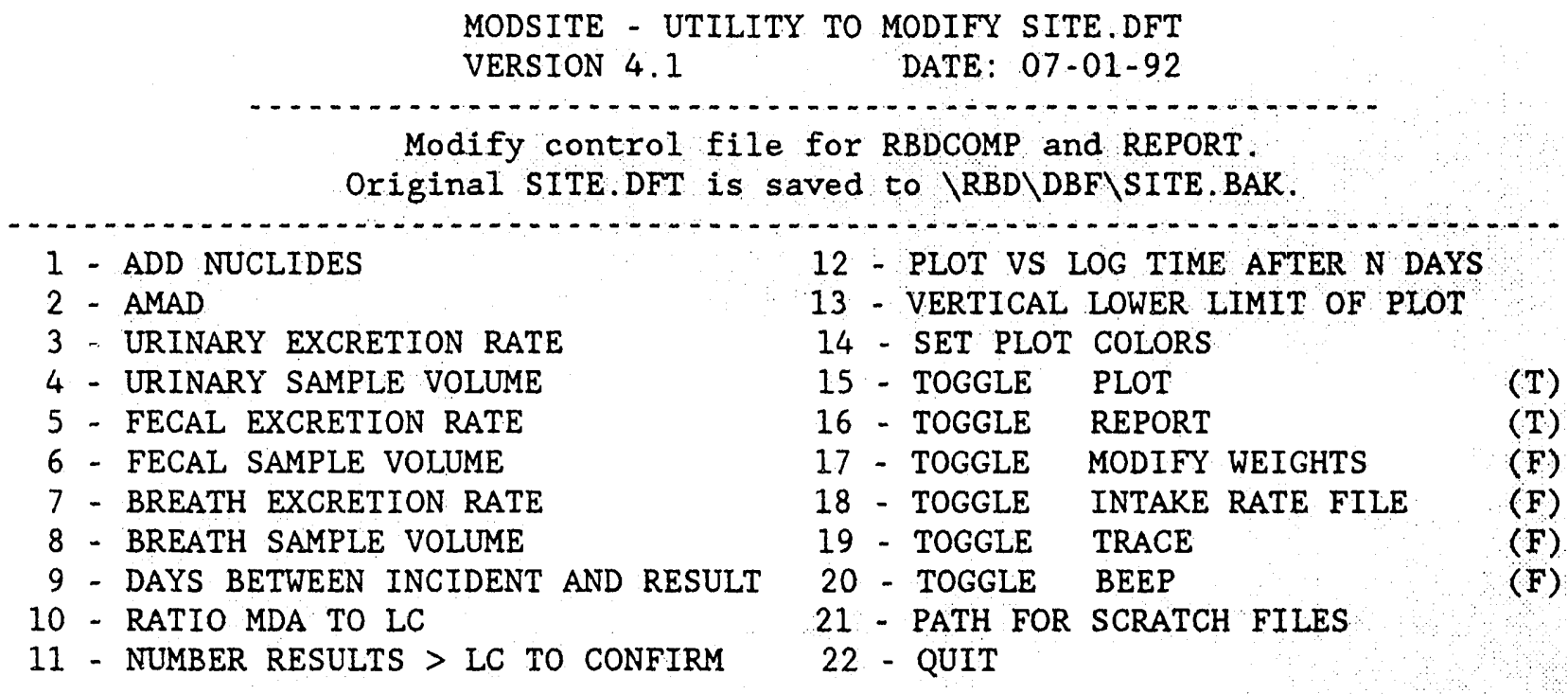

ENTER SELECTION:

Using MODSITE, you can alter switches that control program actions. For example, if the PLOT switch (item 15) is toggled to true (T), you will be able to plot the measurements per unit intake and the expected response per unit intake; if toggled to false $(F)$, no plot is produced. Likewise, if the REPORT switch (item 16) is toggled to true (T), the REPORT program will be run immediately after the RBDCOMP program; if toggled to false (F), the REPORT program is not run. You may run the REPORT program by selecting Generate REPORT from the RBD main menu. 
The SITE.DFT file also contains parameters to control the plot of measurements per unit intake and the expected response per unit intake. Included in these is the duration (in days) when the time axis of the plot switches from linear to logarithmic. Other parameters and switches contained in SITE.DFT are discussed in Sect. 6.

After selecting the RBD main menu item Modify SITEDFT - MODSITE, note whether the PLOT switch (item 15) is set to true (T). If so, nothing needs be done; if not, select item 15 by typing 15 , followed by <Enter >, to toggle the PLOT switch to true (T). Note that when the MODSITE menu is redrawn, the PLOT switch will be modified.

You should then check the setting of the REPORT switch (item 16). RBD is shipped with the REPORT switch set to true (T), as this is the most likely way that RBD will be used. For the lessons, the REPORT switch should be set to false (F). If it is not set to false, select item 16 to toggle the switch to false.

In addition, select item 12 and enter 750., so that all plots in this tutorial will be linear in time. WARNING: MODSITE reads modifications to parameters as character strings without checking the string. Be careful that numbers are typed correctly. To exit MODSITE, select item 22.

You will be asked the question Examine SITEDFT file? By responding Yes, you can verify that the changes have been made to the SITE.DFT file.

Respond Yes in this instance to examine the changes to the SITE.DFT file. Use the $<$ PgUp $>$ and $<\mathrm{PgDn}>$ keys to move around the file. Press $<\mathrm{Esc}>$ to exit to the RBD main menu.

Now that the appropriate changes have been made to the SITE.DFT file, you can continue with the rest of the lessons. No further modifications will be made to the SITE.DFT file. When finished with the lessons, you may customize the SITE.DFT file to suit your needs. 


\subsection{LESSON 2. ENTERING BIOASSAY DATA INTO A BIOASSAY DATA BASE}

Bioassay data are entered into a bioassay' data base using the RBDBASE program. Entry of bioassay data into a bioassay data base is done through RBD main menu item Input Data Base RBDBASE. You can select to enter the data into an existing bioassay data base or into a new bioassay data base, in which case you must supply the name of the data base. The first three characters of the bioassay data base name must be $P B D$.

Select Input Data Base - RBDBASE from the RBD main menu. Create a new bioassay data base $R B D N E W$ by selecting Form New Data Base and typing NEW as shown below.

Radiological Bioassay and Dosimetry Code RBDBASE Version 4.1 07-01-92

RBDTEST.DBF

Form New Data Base

Enter database name: RBDNEW

Data base name begins with RBD. Extension DBF will be added.

Select the RBDNEW data base.

After selecting the $R B D N E W$ data base, the RBDBASE menu appears: 


\begin{tabular}{|l|}
\hline \multicolumn{1}{c|}{$\begin{array}{c}\text { RBDBASE } \\
\text { Version } 4.1\end{array}$} \\
Add Data to Data Base \\
Run AUTOLOAD Utility \\
Enter Exposure History \\
Select Input for RBDCOMP \\
Browse RBDNEW Data Base \\
View Exposure History Data \\
Quit RBDBASE
\end{tabular}

$$
\begin{gathered}
\text { Data base for this session: RBDNEW } \\
\text { Enter assay data into R.BDNEW data base, } \\
\text { RBDBASE MENU }
\end{gathered}
$$

For this tutorial you will be entering data into the RBDNEW data base for an acute exposure to Cs-137. Wh $/ n$ the menu item Add Data to Data Base is selected, the first of two data entry forms, the BIOASSAY DATA ENTRY form, is seen.

To add bioassay records to the data base, select Add Data to Data Base and enter the subje.t name as shown below.

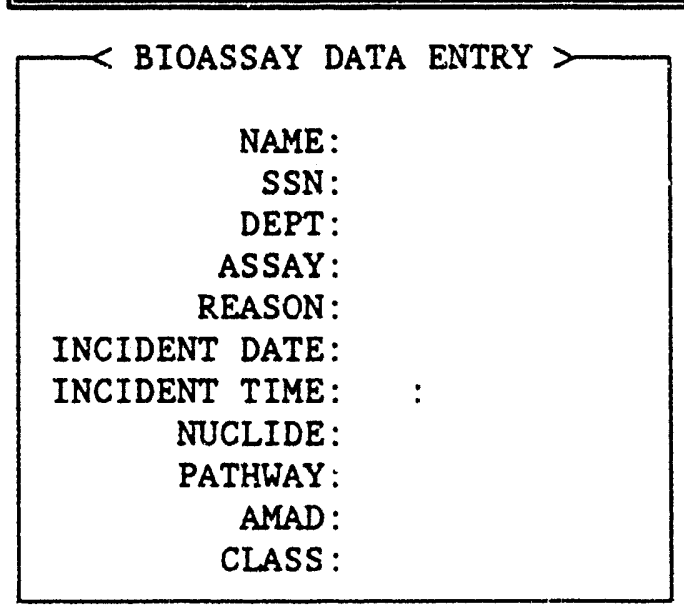

Name: ROENTGEN WILHELM $K$

Enter Name w/o commas; Last First Middle initial 
If this data base had contained bioassay records, you would have seen a list of social security numbers and names from which to select a subject, as shown below for the RBDTEST data base. To enter a new subject into $R B D T E S T$, you would press <Esc > and the name field would appear.

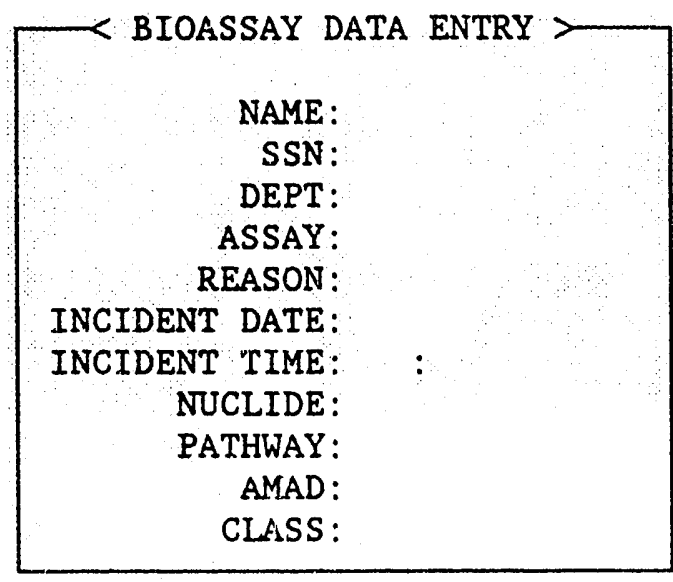

RBDTESI has 30 records; 3 subjects.

\begin{tabular}{|lll|}
\hline SELECT SUBJECT/ & $<$ ESC> TO ENTER NEW SUBJECT \\
\hline ACUTEI131 & FERMI ENRICO & BELV \\
CHRONICI131 & RUTHERFORD ERNST & BELV \\
CHRONICSR90 & HAHN OTTO & BELV \\
\hline
\end{tabular}

Returning to the $R B D N E W$ data entry screen, note that the individual's name should be entered as lasi name, first name, and middle initial, separated by spaces. The name field is 25 characters in lerigis; any name longer than 25 characters will be truncated. The first 12 characters of the last name will appear in the NAME field in the BIOASSAY DATA ENTRY form. The spaces between the last name, first name, and middle initial are replaced by underscores in the data base, because the entire name must be a continuous string.

The BIOASSAY DATA ENTRY form also specifies the individual's social security number (SSN), departmeni, tyne of assay, reason code, incident date and time (for acute intake), nuclide, pathway, AMAD (for intake by inhalation), and class. The cursor will move to each field requesting input. Most responses are sele-ted from items that appear in the upper-right corner. In addition to numbers, upper-case and lower-case letters are allowed in the SSN field, and sorting of records is case sensitive. Pressing <Enter> with no entry in the name, SSN, or department fields will allow you to abort the current data record and return to the menu. The program gives you a chance to correct entries after all fields in the form have been completed. Additional comments and suggestions may appear in the lower-right corner of the screen. 
Enter the social security number (SSN) and department, as shown below. Using the menu bar, select the assay WBC (whole body count) from the list of assays shown at right.

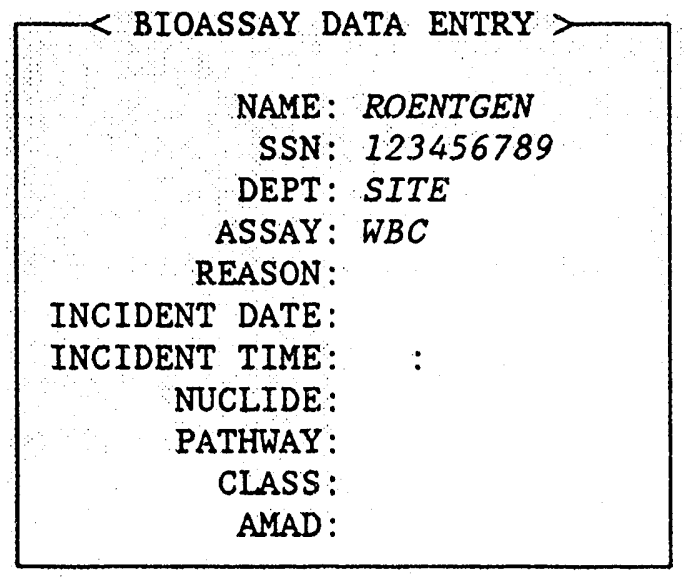

\begin{tabular}{|l|}
\hline U Urine \\
F Feces \\
WBC Whole Body \\
LUN Lungs \\
THY Thyroid \\
BRE Breath
\end{tabular}

You now select the reason code as either incident (1) or routine (3), depending on whether the exposure was acute (incident) or chronic (routine). The incident date and time fields are skipped if the exposure was routine. All dates are entered in the form MM/DD/YYYY. Only the last two digits of the year need be entered. You cannot enter a date beyond the current date obtained from the computer. Note also that the hours and minutes are two separate entry fields.

Select incident for reason and enter an incident date of 01/01/1989 and time of 00:00. 
Next select the nuclide, Cs-137, from the menu that appears at right. This menu is scrollable. The arrow keys and the $\langle\mathrm{PgUp}\rangle$ and $\langle\mathrm{PgDn}\rangle$ keys can be used to move about the menu.

\begin{tabular}{|c|} 
BIOASSAY DATA ENTRY > \\
NAME: \\
SSOENTGEN 123456789 \\
DEPT: $S I T E$ \\
ASSAY: $W B C$ \\
REASON: 1 \\
INCIDENT DATE: $01 / 01 / 1989$ \\
INCIDENT TIME: $00: 00$ \\
NUCLIDE: $C S-137$ \\
PATHWAY: \\
AMAD: \\
CLASS:
\end{tabular}

Now select the route of intake or pathway, inhalation, from the menu at right.

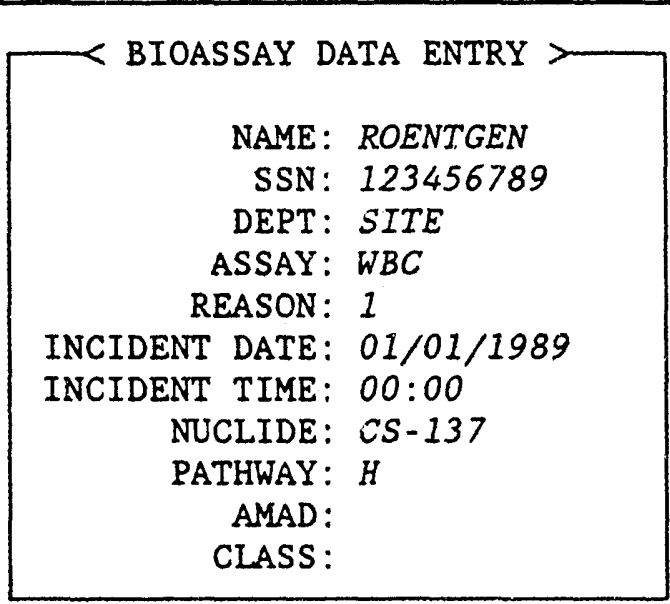

\begin{tabular}{|ll|}
\multicolumn{2}{c}{ SELECT NUCLIDE } \\
AM-241 & Americium \\
C-11 & Carbon \\
C-14 & Carbon \\
CM-242 & Curlum \\
CM-244 & Curium \\
CO-57 & Cobalt \\
CO-58 & Cobalt \\
CO-60 & Cobalt \\
CS -134 & Cesium \\
CS-137 & Cesium \\
EU-152 & Europium \\
EU-154 & Europium \\
EU-155 & Europium \\
H-3 & Tritium Vapor \\
I-125 & Iodine \\
I-129 & Iodine \\
I-131 & Iodine \\
$I-135$ & Iodine \\
NA-22 & Sodium \\
NA -24 & Sodium \\
NI -63 & Nickel \\
\hline
\end{tabular}

Now select the route of intake or pathway, inhalation, from the menu at ight. 
The activity median aerodynamic diameter (AMAD) characterizes the particle size of the inhaled aerosol. You can supply a value or press <Enter > to accept the default value of $1.00 \mu \mathrm{m}$. Following this, you select the inhalation class.

Press <Enter> to accept the default value for AMAD. Select the inhalation class from the list at right. For this case there is only one class, D (Day). Press $<$ Enter $>$ to select it.

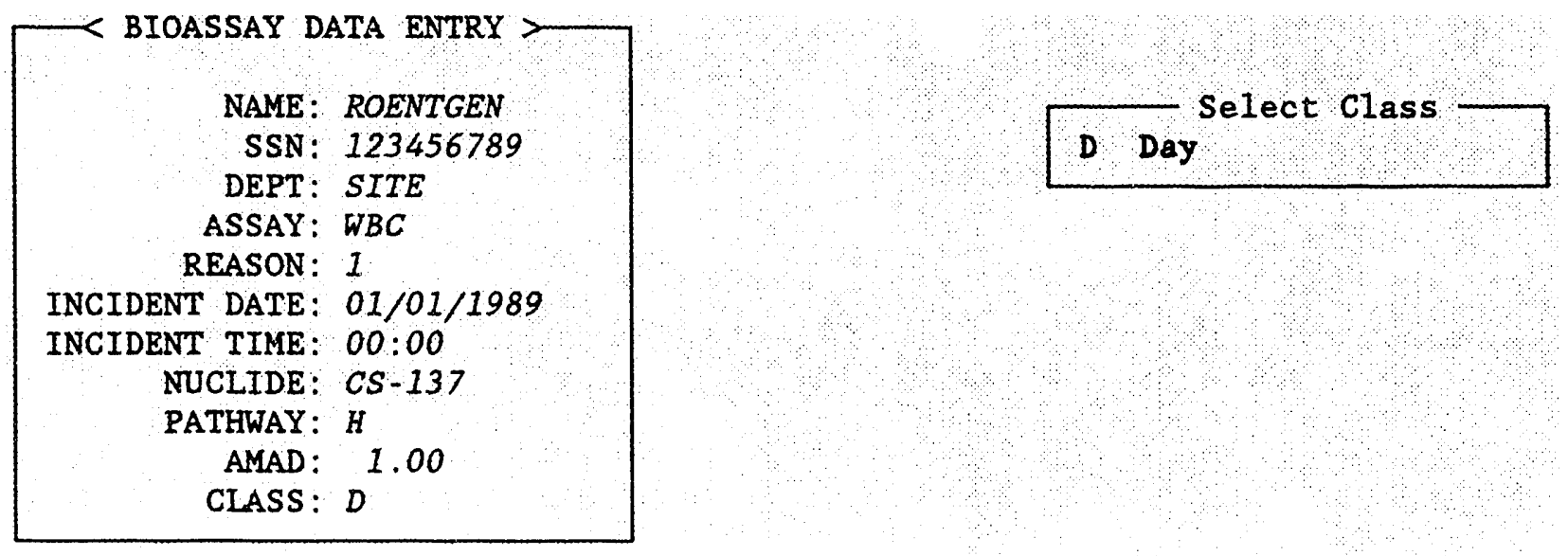

When the first form is complete, you are asked Do you want to exit this form? You respond No if you wish to correct any entries in the first form. You respond Yes if you wish to go on to the next form. Yes is the default response. If you do need to make corrections, the program retains your previous entries. You simply press <Enter > to accept correct entries.

After any corrections, respond Yes to exit the first data entry form.

Upon exiting the first form, a second form appears in the lower-left corner of the screen with a menu of units for the selected assay in the upper-right corner of the screen. The second form requests measurements (date and time of sample and measured value) pertinent to the individual and exposure described on the first form. In the case of incident exposure, the measurement date/time must be after the incident date/time. If a date is out of range, an error message is printed, and you must correct the entry. The units of the measurement and MDA are selected from the menu, which appears in the upper-right corner. You must select one of the units shown. When the form is completed, you can enter a comment line (up to 41 characters). The comment is appended to the record and is ignored by RBDCOMP. Finally, you are asked Do you want to exit this form? You respond No if you wish to correct any entries. You respond Yes if you wish to either enter another measurement or to return to the first form and enter information for another individual. Yes is the default response.

There are two types of second form: one for in vivo measurements and one for in vitro measurements. 
For in vivo assay (e.g., whole body count, as used in this example) the form reads:

BIOASSAY DATA ENTRY

NAME : ROENTGEN

SSN: 123456789

DEPT: SITE

ASSAY: WBC

REASON: 1

INCIDENT DATE: 01/01/1989

INCIDENT TIME: 00:00

NUCLIDE: $C S-137$

PATHWAY: $H$

AMAD: 1.00

CLASS: $D$

\begin{tabular}{|c|c|c|}
\hline M & $\begin{array}{l}\text { Assay Units } \\
\text { micro } \mathrm{Ci}\end{array}$ & ( $\mu \mathrm{Ci})$ \\
\hline $\mathrm{N}$ & nano $\mathrm{Ci}$ & (nCi) \\
\hline $\mathbf{P}$ & pico Ci & $(\mathrm{pCi})$ \\
\hline D & dpm & \\
\hline B & becquerel & (Bq) \\
\hline$U$ & micro gram & $(\mu \mathrm{g})$ \\
\hline
\end{tabular}

Enter In-Vivo Results

Whole body measurements are normally not used with $\mathrm{H}-3$ and C-14.

Two results greater than critical limit MDA/2 confirm the intake.

Add any comments: DISCOVERED XRAYS

Do you want to exit this form? $(Y / N) Y$

Enter the WBC measurement date, time, result, and MDA for the Cs-137 incident as shown above. 
For in vitro assay (e.g., urinalysis) the form would have read:

\section{EXAMPLE ONLY - DO NOT ENTER THESE DATA!}
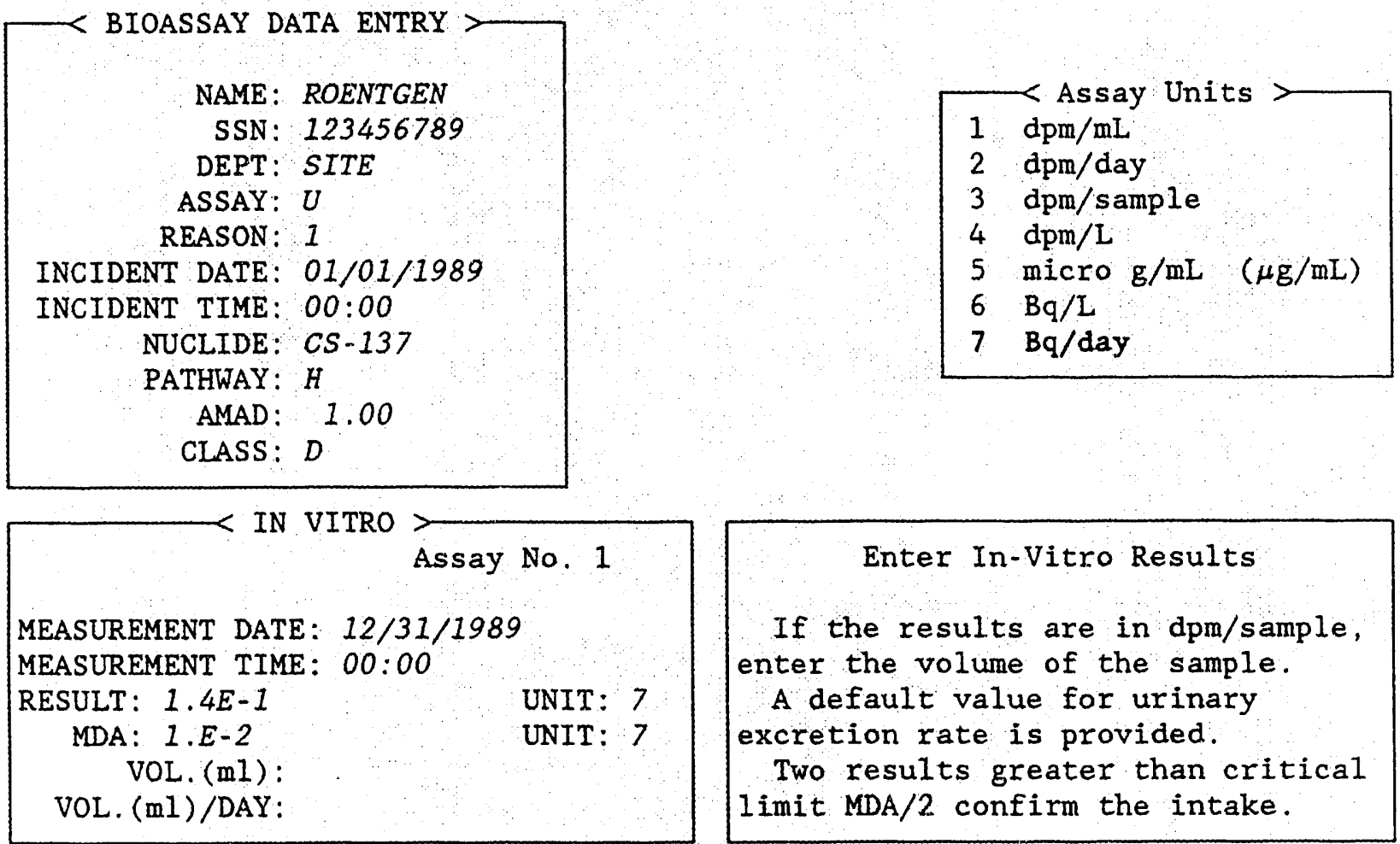

Add any comments:

Do you want to exit this form? (Y/N) $Y$

\section{EXAMPLE ONLY - DO NOT ENTER THESE DATA!}

Some in vitro assays will require the sample volume and/or the excretion rate per day. A default value of excretion rate is provided, which you can accept by pressing $<$ Enter $>$ or override by entering a value.

After all corrections are made to the second form, the record is added to the bioassay data base (in this example, $R B D N E W$ ). Another measurement can then be entered for the same exposure by responding Yes, which is the default answer, to the question ENTER ANOTHER MEASUREMENT? This response will open an empty second form for entering another measurement. A new record is added to the data base whenever you exit the form.

Because there is only one measurement for the Cs-137 example, respond No to the question ENTER ANOTHER MEASUREMENT? 
At this point your choices are (1) to enter data for a new assay or nuclide, (2) to enter data for a new individual, or (3) to end data entry. Choose 1 for new assay or new nuclide for the same individual. Choose 2 to enter data for another individual. Choices 1 and 2 will return you to the first form to continue data entry. When completed with all data entry, choose (3) to end data entry and exit to the RBDBASE menu.

For this tutorial, choose option 1 and enter a single chronic exposure to Cs-137 for this same individual. Enter exactly the same data as for the acute case, but select reason code 3 (routine). Proceed to the second entry form and enter a single measurement on 12/31/1989 at time 00:00 of $52.0 \mathrm{~Bq}$ with an MDA of $10.0 \mathrm{~Bq}$. When finished, select option 3 to exit the data entry. Then press $<$ Esc $>$ twice to return to the RBD main menu.

You can browse the records just entered to check their accuracy (see the following lesson). To prevent inadvertent modification of records, we have designed the RBDBASE program so that records cannot be edited in the browse feature. ${ }^{1}$ Records can be edited only by using the data base utility EDITDBF. When checking of the records is complete, you can enter exposure history records and/or select records for input to the RBDCOMP program.

${ }^{1}$ To allow editing in the browse feature of the RBDBASE, run SETUPRBD and respond Yes to the query Edit in Browse of RBDBASE ? Use extreme caution in editing data base records in the browse feature of RBDBASE, as you will not be prompted, when exiting the data base, that modifications have been made. 
5.3 LFSSON 3. BROWSING RECORDS IN A BIOASSAY DATA BASE

To browse the records of an existing bioassay data base, select Input Data Base RBDBASE from the RBD main menu and select the RBDTEST data base. Select Browse RBDTEST Data Base from the RBDBASE menu, as shown below.

Select item using $\uparrow \downarrow$ and $\longleftrightarrow \downarrow$

\begin{tabular}{|l|}
\hline \multicolumn{1}{|c|}{$\begin{array}{c}\text { RBDBASE } \\
\text { Version } 4.1\end{array}$} \\
Add Data to Data Base \\
Run AUTOLOAD Utility \\
Enter Exposure History \\
Select Input for RBDCOMP \\
Browse RBDTEST Data Base \\
View Exposure History Data \\
Quit RBDBASE
\end{tabular}

Data base for this session: RBDTEST

Browse the bioassay records in RBDTEST data base.

\section{RBDBASE MENU}


You should see the following screen after selecting Browse RBDTEST Data Base:

\begin{tabular}{|c|c|c|c|c|c|c|c|c|c|}
\hline \multicolumn{3}{|c|}{$\begin{array}{l}\text { Browsing RBDTEST file } \\
\text { Press <Esc> to exit. }\end{array}$} & \multirow[b]{2}{*}{$\mathrm{Cl}$} & \multirow[b]{2}{*}{ Assay } & \multirow[b]{2}{*}{ Path } & \multicolumn{2}{|c|}{ Arrow keys } & \multicolumn{2}{|c|}{$\begin{array}{l}\text { Record } 4 / 30 \\
\text { are operative. }\end{array}$} \\
\hline SSN & Dept & Nuclide & & & & AMAD & Reason & Inc_Date & hr min \\
\hline ACUTEI131 & |BELV| & $\mid I-131$ & D & THY & $\mid H$ & 1.00 & 11 & $02 / 01 / 1989$ & $0: 0$ \\
\hline ACUTEI 131 & BELV & $I-131$ & $D$ & THY & $\mathrm{H}$ & 1.00 & 1 & $02 / 01 / 1989$ & $0: 0$ \\
\hline ACUTEI 131 & BELV & $\mathrm{I}-131$ & D & $\mathrm{U}$ & $\mathrm{H}$ & 1.00 & 1 & $02 / 01 / 1989$ & $0: 0$ \\
\hline ACUTEI13I & BELV & $I-131$ & D & $\mathrm{U}$ & $\mathrm{H}$ & 1.00 & 1 & $02 / 01 / 1989$ & $0: 0$ \\
\hline ACUTEI131 & BELV & $I-131$ & D & $\mathrm{U}$ & $\mathrm{H}$ & 1.00 & 1 & $02 / 01 / 1989$ & $0: 0$ \\
\hline ACUTEI131 & BELV & $I-13 I$ & D & $\mathrm{U}$ & $\mathrm{H}$ & 1.00 & $\overline{1}$ & $02 / 01 / 1989$ & $0: 0$ \\
\hline ACUTEI131 & BELV & $I-131$ & D & $\mathrm{U}$ & $\mathrm{H}$ & 1.00 & 1 & $02 / 01 / 1989$ & $0: 0$ \\
\hline ACUTEI131 & BELV & $I-131$ & D & $\mathrm{U}$ & $H$ & 1.00 & 1 & $02 / 01 / 1989$ & $0: 0$ \\
\hline ACUTEI131 & BELV & $\mathrm{I}-131$ & D & $\mathrm{U}$ & $\mathrm{H}$ & 1.00 & 1 & $02 / 01 / 1989$ & $0: 0$ \\
\hline ACUTEI 131 & BELV & $I-131$ & D & $U$ & $\mathrm{H}$ & 1.00 & 1 & $02 / 01 / 1989$ & $0: 0$ \\
\hline ACUTEI131 & BELV & $I-131$ & D & U & $\mathrm{H}$ & 1.00 & 1 & $02 / 01 / 1989$ & $0: 0$ \\
\hline ACUTEI131 & BELV & $I-131$ & D & $\mathrm{U}$ & $\mathrm{H}$ & 1.00 & 1 & $02 / 01 / 1989$ & $0: 0$ \\
\hline ACUTEI131 & BELV & I- 131 & D & U & $\mathrm{H}$ & 1.00 & 1 & $02 / 01 / 1989$ & $0: 0$ \\
\hline ACUTEI 131 & BELV & $I-131$ & D & 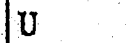 & $\mathrm{H}$ & 1.00 & 1 & $02 / 01 / 1989$ & $0: 0$ \\
\hline CHRONICI 131 & BELV & $\mathrm{I}-131$ & D & AAA & $\mathrm{H}$ & 0.00 & 3 & 11 & $0: 0$ \\
\hline CHRONICI 131 & BELV & $I-131$ & D & THY & $\mathrm{H}$ & 1.00 & 3 & 1 & $0: 0$ \\
\hline CHRONICI 131 & BELV & $\mid I-131$ & D & THY & $\mathrm{H}$ & 1.00 & 3 & 1 & $0: 0$ \\
\hline
\end{tabular}

In this example, names are used in the SSN field (e.g., ACUTEI131 and CHRONICI131) instead of social security numbers. The SSN would normally be a nine-digit number.

You can move around in the browse mode using the arrow keys, the $\langle\mathrm{PgUp}\rangle,\langle\mathrm{PgDn}\rangle$, $<$ Home $>$, and $<$ End $>$ keys. To move to the far left field, press $<$ Ctrl $><$ Home $>$; to the far right field, press $<\mathrm{Ctrl}><$ End $>$; to the top, press $<\mathrm{Ctrl}><\mathrm{PgUp}>$; and to the bottom, press $<\mathrm{Ctrl}>$ $<\mathrm{PgDn}>$. Pressing the help key (the $<\mathrm{F} 1>$ key) while browsing the bioassay data base will display a table of codes used in the records.

Practice moving around the RBDTEST data base using the above noted keys. Press <Esc $>$ three times to return to the RBD main menu. 
To edit or delete records from a bioassay data base, you exit the RBDBASE menu by pressing $<$ Esc $>$ until the RBD main menu appears. You select Data Base Utility - EDITDBF from the main menu. You select the desired bioassay data base and the Browse/Edit feature for the data base. You can edit records in the data base by moving the cursor to the appropriate field, pressing <Enter >, editing the field, and pressing $<\mathrm{E}: \mathrm{er}>\mathrm{a}$ second time. The edit feature is normally in typeover mode; you press <Insert > for insert mode. To mark a record for deletion, you align the cursor on the record and press the $<$ F3 $>$ key. $<$ DELETED $>$ will appear in the header indicating the record is marked for deletion. Pressing the $<F 3>$ key the second time removes the mark for deletion. The record will be deleted on exiting the Browse/Edit feature. You press $<$ Esc $>$ after completing modifications. If modifications have been made to the data base, the program asks whether you wish to save the modified records. If so, you respond Yes. You can select the browse feature of EDITDBF to examine the modified records. You press <Esc > twice to exit the EDITDBF utility and return to the RBD main menu.

Extreme caution must be used in editing data in the bioassay data base.

You will now examine the exposure history records in the RBDTEST data base.

From the RBD main menu, select Input Data Base - RBDBASE. Then select the RBDTEST data base and select View Exposure History Data to browse the RBD bioassay data base RBDTEST for exposure history records. Select ALL HISTORY RECORDS to see all the exposure history records in this bioassay data base.

You should see the following screen after selecting View Exposure History Data and selecting All History Records:

\begin{tabular}{|c|c|c|c|c|c|c|c|}
\hline \multicolumn{4}{|c|}{$\begin{array}{l}\text { Exposure History Records: RBDTEST } \\
\text { Press <Esc }>\text { to exit. }\end{array}$} & \multicolumn{4}{|c|}{ Record 1/4 } \\
\hline SSN & Dept & Nuclide & C1 & Assay & Date & $\mathrm{hr}$ & $\min$ \\
\hline CHRONICI131 & BELV & $I-131$ & D & $\mathrm{AAA}$ & $01 / 01 / 1989$ & 0 & 0 \\
\hline CHRONICI131 & BELV & I-131 & D & $\mathrm{ZZZ}$ & $01 / 01 / 1990$ & 0 & 0 \\
\hline CHRONICSR9O & BELV & SR-90 & $\mathrm{Y}$ & AAA & $01 / 01 / 1989$ & 0 & 0 \\
\hline CHRONICSR90 & BELV & SR - 90 & $\mathrm{Y}$ & $\mathrm{ZZZ}$ & $12 / 31 / 1989$ & 24 & 0 \\
\hline
\end{tabular}

Press < Esc > to return to the RBDBASE menu, and select View Exposure History Data again. To view the exposure history records for a single SSN, select SINGLE SSN from the browse Exposure History menu. 
A list of social security numbers and names for each subject in the data base appears. Move the menu bar to the subject desired and press <Enter $>$. You will see the exposure history records for that subject. Note that exposure history records contain assay codes AAA and ZZZ, which indicate start and end of exposure to that nuclide, with the date field being the starting or ending date.

Select CHRONICI131 from the list of social security numbers and examine the exposure history records. Press $<$ Esc $>$ four times to return to the RBD main menu.

The RBDTEST data base contains three sets of bioassay records: an acute exposure to I-131, a chronic exposure to I-131, and a chronic exposure to Sr-90. The bioassay data for the acute I-131 exposure are taken from NUREG/CR-4884 (ref. 8). Both of the chronic exposure cases contain exposure history records.

Practice using the browse feature to examine the records entered into the bioassay data base $R B D N E W$ in Lesson 2. When finished, press $<\mathrm{Esc}>$ three times to return to the RBD main menu. 


\subsection{LESSON 4. EXTRACTING RECORDS FROM A BIOASSAY DATA BASE AND COMPUTING INTAKE AND DOSE USING RBDCOMP}

Once bioassay data sive been entered into the bioassay data base, they may be extracted in various ways for processing through RBDCOMP. In this lesson, you will extract records from the $R B D T E S T$ data base and compute the intake and dose using RBDCOMP.

Select Input Data Base - RBDBASE from the RBD main menu and select the RBDTEST bioassay data base. The RBDBASE menu will appear. Move the cursor to the menu item Select Input for RBDCOMP and press <Enter >.

The following menu will appear:

Select Data From RBDTEST For Input To RBDCOMP.

Select Data Using $\uparrow \downarrow \&<-$

SINGLE SSN

MULTIPLE SSN

ALL DATA

QUIT TO PREVIOUS MENU

Extract bioassay records from RBDTEST data base by Social Security Number.

Records may be selected for a single SSN or for multiple SSNs. All records in the data base may be selected using ALL DATA

Select SINGLE SSN to extract records for a single individual. 
You should see the following menu upon selecting SINGLE SSN:

RBDTEST has 30 records; 3 subjects

SELECT SSN OF INTEREST/<ESC> TO EXIT

ACUTEI131 FERMI ENRICO BELV

CHRONICI 131 RUTHERFORD ERNST BELV

CHRONICSR90 HAHN OTTO BELV

- - - - END - - - END - - - - END - - - - END - - - -

Each menu item shows the SSN, name, and department code for individuals in the data base. The SSN would normally be a nine-digit number. Here names are used to identify the test cases. The bioassay data for the acute I-131 exposure are taken from NUREG/CR-4884 (ref. 8).

\section{Select ACUTEI131 FERMI_ENRICO BELV.}

You are then asked, as shown on the following page, for a name for the bioassay records file. The extension DAT will be appended to the name. The name entered here, with various extensions, will be used for all subsequent files generated using these bioassay records. This name is restricted to eight characters, including the Army dosimetry account code. The Army dosimetry account code, assumed in this tutorial to be $D E M O$, will appear in the first part of the name field.

Enter the name $A I O D$ to make the DAT file name DEMOAIOD. 
Select Data From RBDTEST For Input To RBDCOMP.

\begin{tabular}{|l|}
\hline Select Data Using $+\downarrow \&<-\mid$ \\
\hline SINGLE SSN \\
MULTIPLE SSN \\
ALL DATA \\
QUIT TO PREVIOUS MENU \\
\hline
\end{tabular}

SSN Selected: ACUTEI131

14 Records were found.

Enter name for bioassay record filn; the extension DAT will be added: DEMOAIOD

The RBDBASE program extracts the selected records, sorting them into the order required by RBDCOMP. The bioassay records are written to the file DEMOAIOD.DAT in the format specified in Appendix A. If the file already exists, you are asked whether it can be written over.

After successfully generating the bioassay records file, exit the RBDBASE menu by moving the menu bar to Quit RBDBASE (or pressing $Q$ ).

Next you select Bioassay Program - RBDCOMP from the RBD main :nenu to compute intake and dose.

Select Bioassay Program - RBDCOMP from the RBD main menu.

You will see a list of bioassay records files (DAT files). You select the desired file from the list by moving the menu bar to the item and pressing <Enter $>$. This marks the item with arrows. If you press <Enter> a second time, the choice is canceled.

¿Iove the menu bar to entry DEMOAIOD.DAT and press <Enter $>$ to select it. Press $<$ Enter > a second time and note that it is canceled. Press <Enter > to select again.

You may select any number of files to be processed by RBDCOMP. If there are more than nine files to choose from, you can proceed to the next screen by selecting the line Next Screen for more files, by pressing $N$, or by pressing $\langle\operatorname{PgUp}\rangle$ or $\langle\operatorname{PgDn}\rangle$, which have the same action as pressing $N$. 
On each selection screen you will see the selection Execute RBDCOMP Program. After selecting all files to be run through the bioassay program, you move the menu bar to the line Execute RBDCOMP Program and press the <Enter > key (or press $E$ ). This executes RBDCOMP to process the selected DAT files. A separate output records file, with extension DBS, is produced for each DAT file. The format for the output records file is specified in Appendix B.

Check that DEMOAIOD.DAT (and no other file) is selected. Then select Execute RBDCOMP Program (or press $E$ ).

If an RBDCOMP output file (LOG file) exists with the name of the bioassay records file, RBDCOMP will ask you whether to replace it. If you have previously computed intake and dose for this bioassay records file, the output files (LOG and DBS files) may exist in the subdirectory WORK. You may want to archive the output files using the FILMAN utility as RBDCOMP will write over the existing files.

The RBDCOMP bioassay program prints a header giving the version and date of the program, the date of the computation, and the name of the bioassay records file. You must specify the display option: (0) Batch, which is used for batch processing; (1) Intake and Dose, which gives intake and dose information but no summary of the bioassay record; or (2) Full, which gives a summary of the bioassay records in addition to the computed intake and dose values. Display option 1 is the default. No plots are available for display option 0 . For display options 1 and 2 , if the video adapter and monitor support graphics and the PLOT switch in SITE.DFT is set to true (T), RBDCOMP will ask whether you wish to see a plot comparing measurements per unit intake and the expected response per unit intake.

Press <Enter > to select the default display option (1).

If the bioassay program detects that there are routine records in the input, the program will ask for a starting and ending date, in the form YYYYMMDD, to bracket the period of the routine measurements. This time period is referred to as the measurement window. You can also enter the year in the form YYYY followed by a duration in years for considering measurements over an integral number of years.

Because there are no routine records, you will not be asked to enter a measurement window for this example. 
Finally, RBDCOMP asks for a starting and ending date, in the form YYYYMMDD, to define the period over which the intake will be computed. This time period is referred to as the intake window. Note that the intake window should be contained within the measurement window. Since one normally evaluates intakes annually, the intake window would be 1 year. You can also enter the year in the form YYYY followed by a duration in years for considering measurements over an integral number of years.

Enter the year 1989, with a duration of 1 year, for the intake window.

To this point, the screen display for this example (bioassay records file DEMOAIOD.DAT) will appear as follows, with user responses shown in italics.

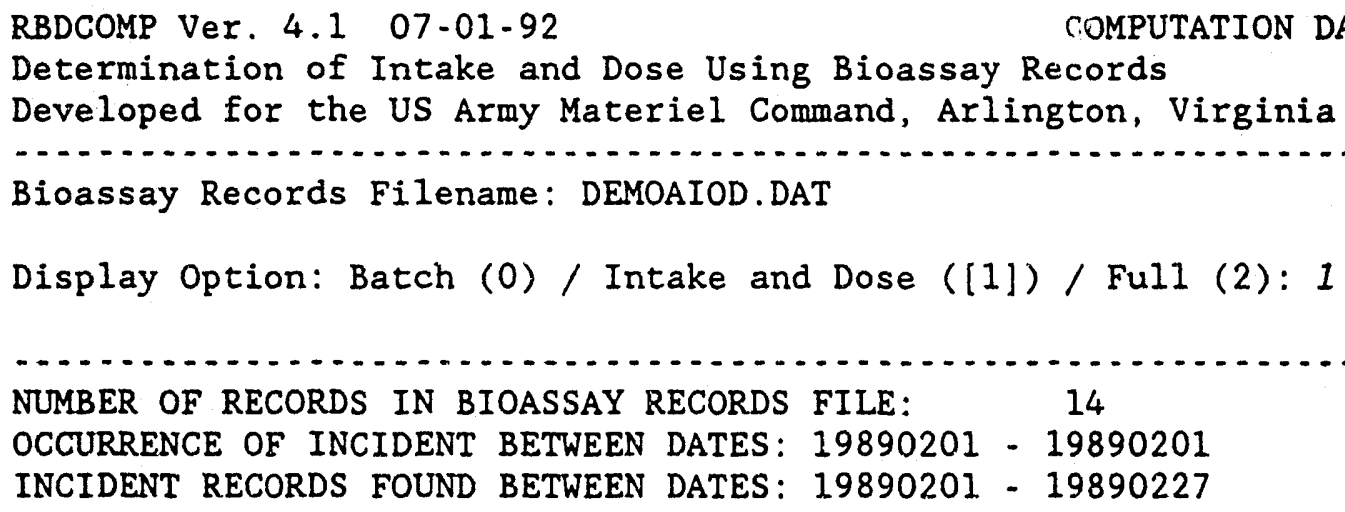

Enter Intake Period -. Time Period for Intake and Dose Assessment

Entering just the year [YYY] followed by duration in years implies Jan $1(00: 00)$ to Dec $31(24: 00)$ for the number of years specified.

Enter Beginning Intake Date [YYYYMMD] or [YYYY]: 1989 Enter Duration in Years: 1

If you select display option 1, the default response, RBDCOMP will display the intake and dose for each assay for a given SSN, nuclide, class, and reason code and pause to allow you to examine the results. Here you have entered a starting date for computing intake of 1989 (assumes 19890101) with a 1 -year duration. Incident cases will be evaluated only if the incident date falls within this intake window. There will be two sets of results for the example DEMOAJOD - one for urinalysis and one for thyroid counts. The intake and committed dose are shown using both SI and conventional units. 
If you have not already done so, press $<$ Enter $>$ to continue as prompted on the screen display. You should now see the urinalysis results for DEMOAIOD which are shown below.

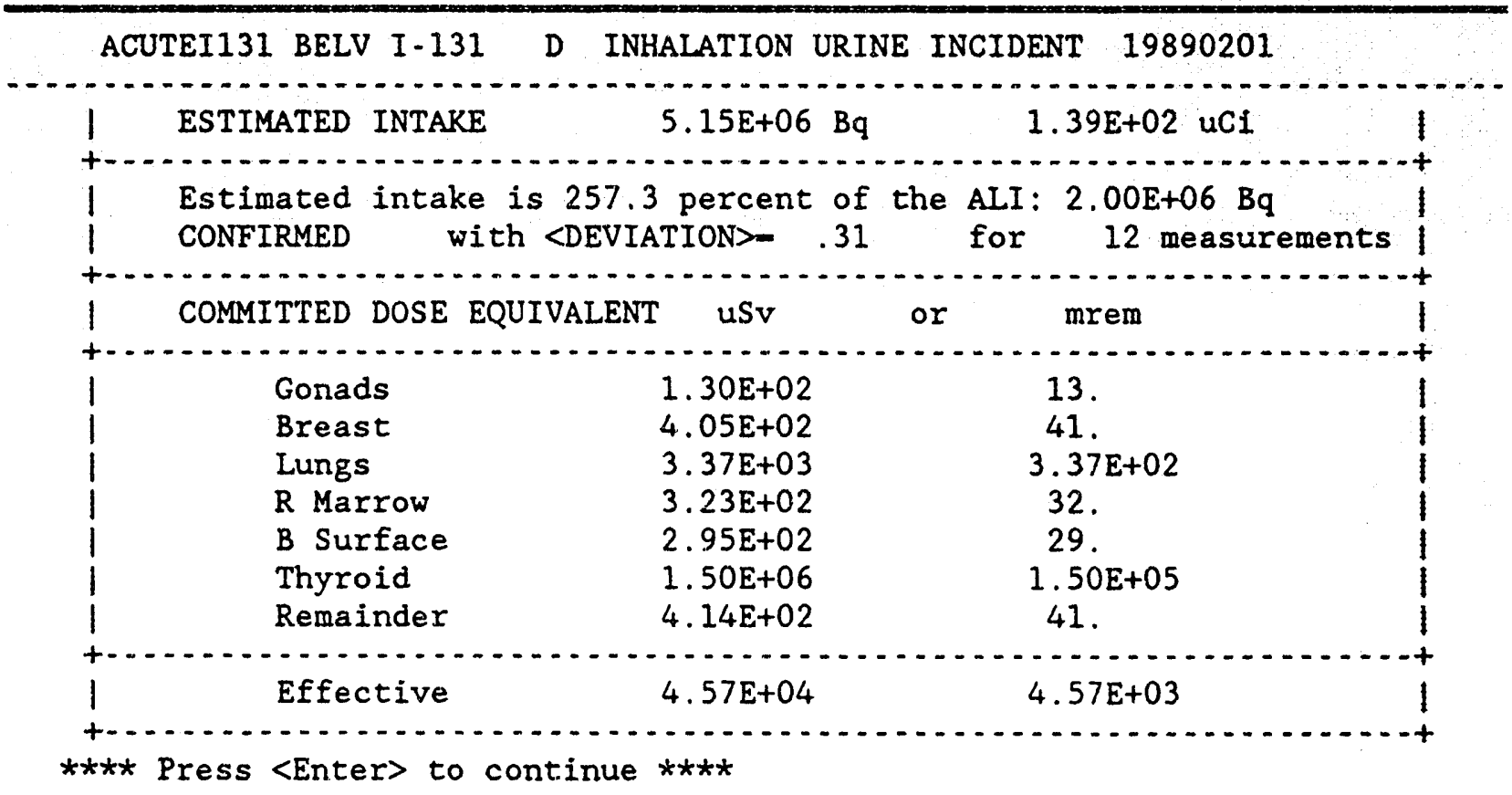

After the results for urinalysis are displayed, press <Enter $>$ to continue.

If your monitor supports graphics and the PLOT switch in SITE.DFT is set to true (T), the program will ask Plot measurements per unit intake and unit response function? For this example, the unit response function is the expected excretion rate per unit intake. You can modify the default minimun and maximum values for the time and response axes. If the plotting option is unavailable or you respond No (the default response) to the prompt, RBDCOMP will continue to the next case (SSN, nuclide, class, and reason code).

Respond Yes to see the plot of measurements per unit intake and the expected I-131 excretion rate per unit intake. Accept the default minimum and maximum values for the time and response axes.

The plot of the urine measurements per unit intake and the expected excretion rate per unit intake is shown in Fig. 4. 


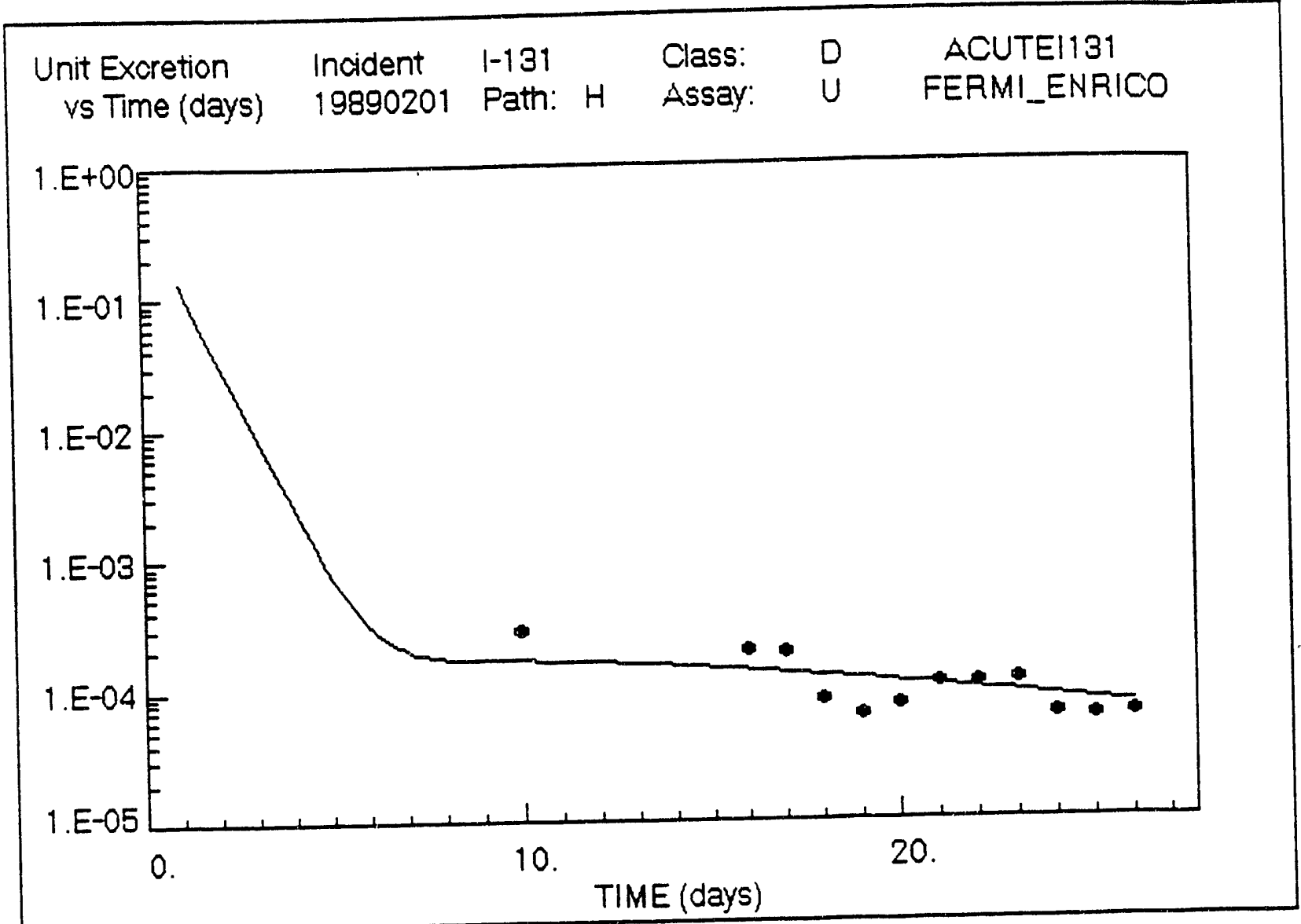

Fig. 4. Observed and expected urinary excretion for acute exposure to ${ }^{131} \mathrm{I}$. 
Press <Enter > to clear the plot.

You will now see the estimated intake and committed dose computed using the thyroid counts.

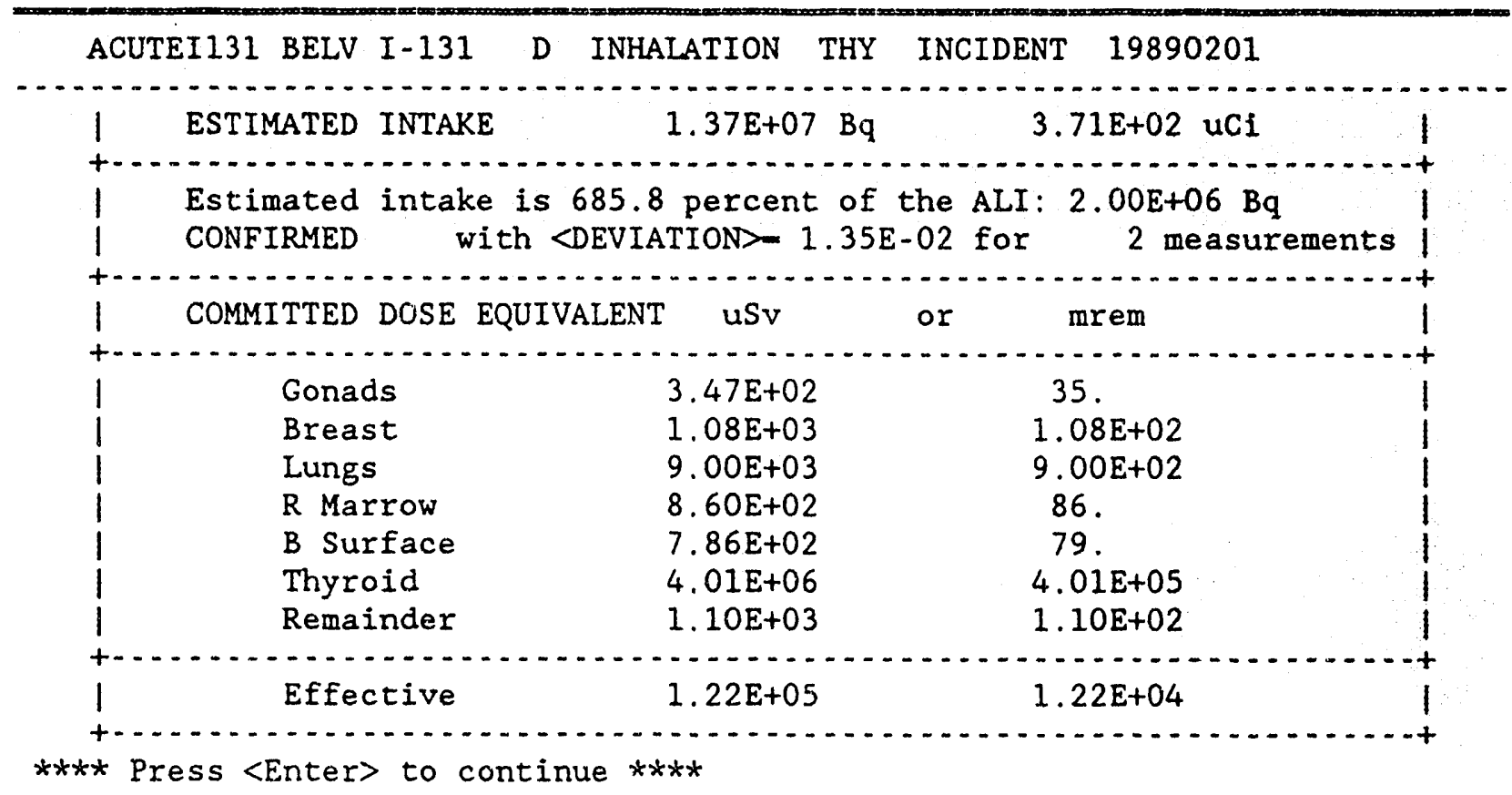

Press <Enter > to continue. Respond Yes to Plot measurements per unit intake and unit response function? Accept the default minimum and maximum values for the time and response axes.

The plot of thyroid measurements per unit intake and the expected thyroid activity per unit intake is shown in Fig. 5.

Press < Enter $>$ to clear the plot. 


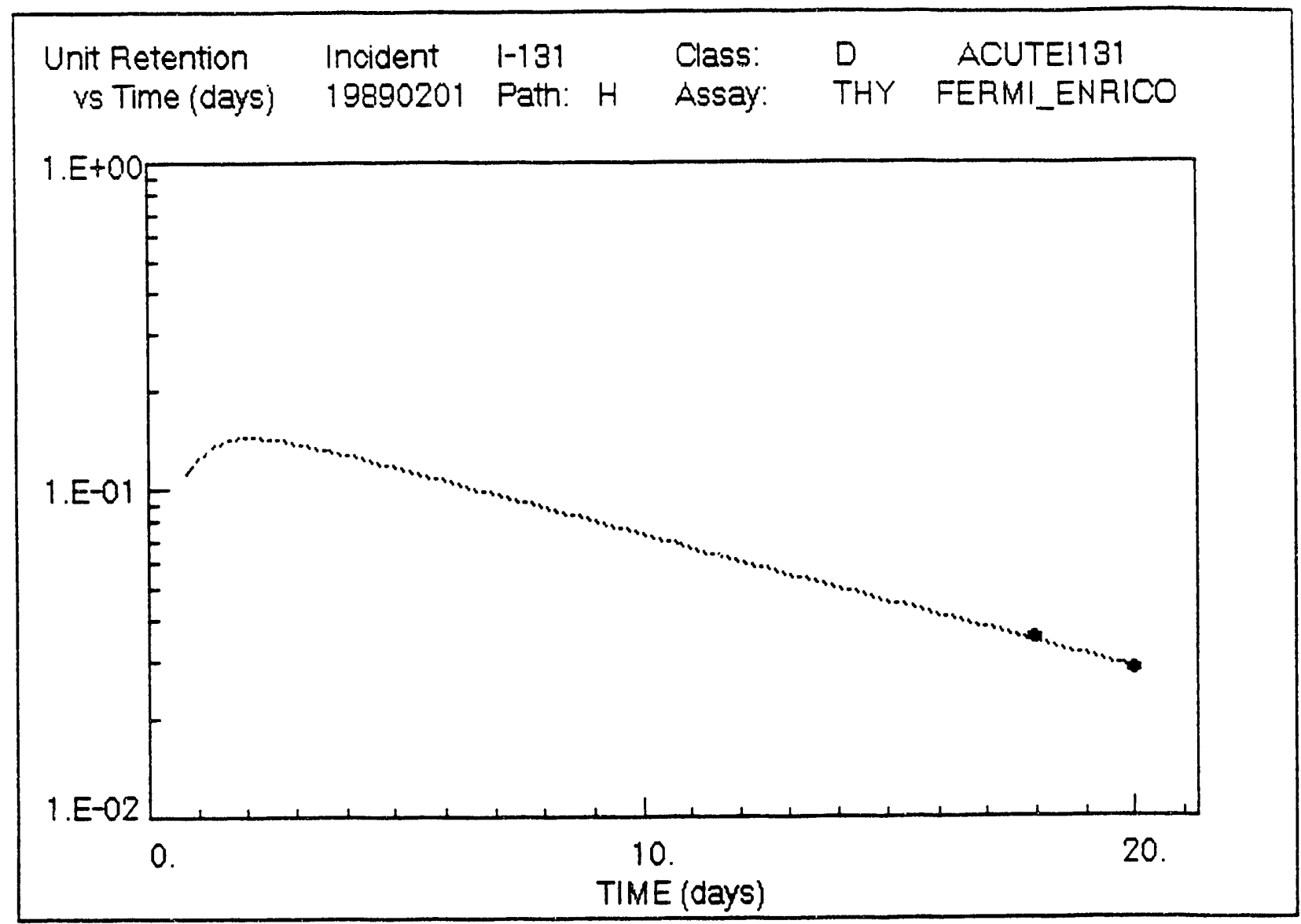

Fig. 5. Observed and expected thyroid retention for acute exposure of ${ }^{131} \mathrm{I}$. 
When the estimation of intake is based on two or more assays, RBDCOMP displays the summary table. You will see the following summary table for the I-131 incident exposure.

$* * * * *$ SUMMARY TABLE $* * * *$

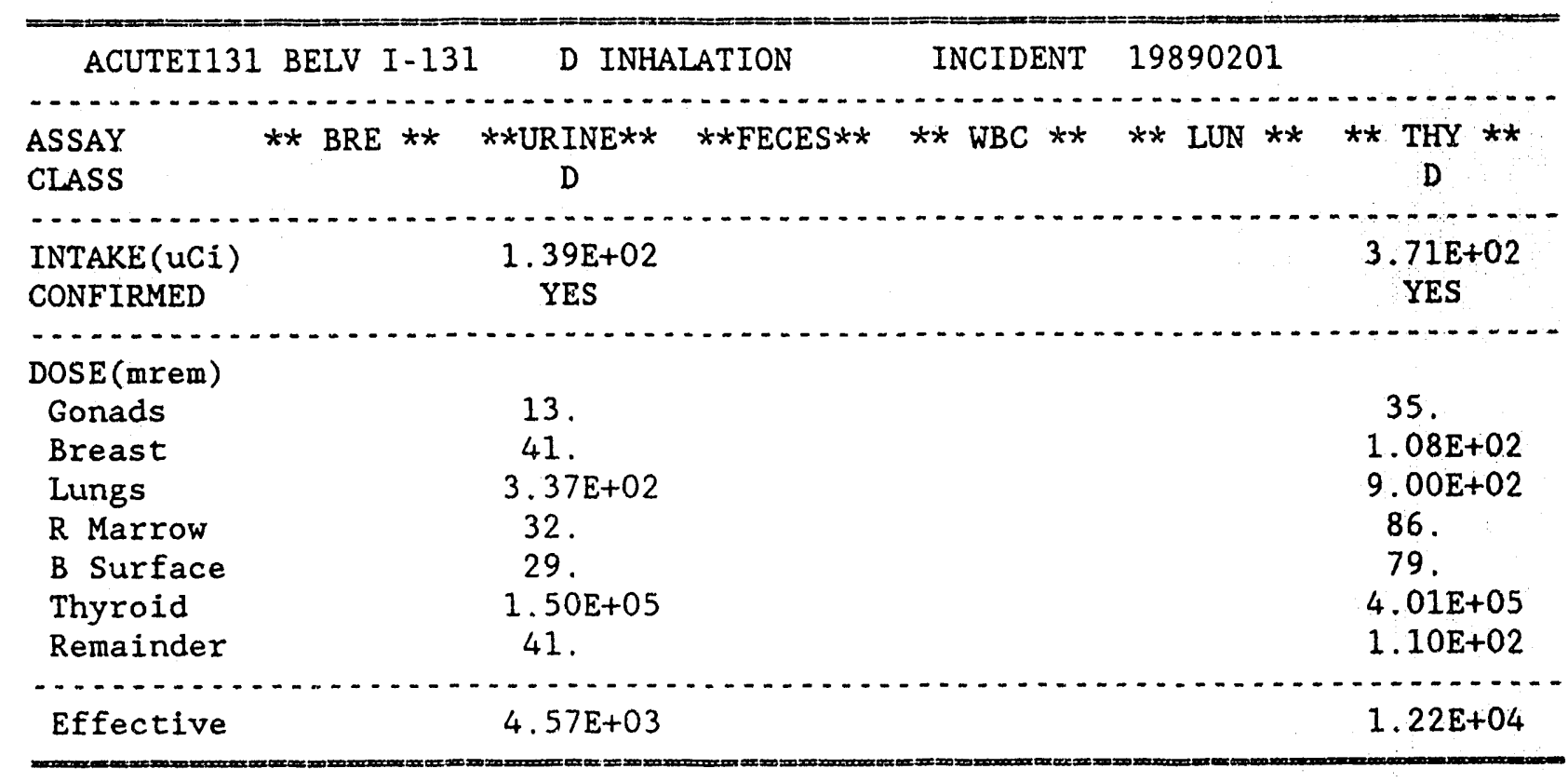

$* * * *$ Press <Enter> to continue $* * * *$

Press <Enter > to clear the summary table and return to the RBD main menu. 
If routine records had been detected in the bioassay records file, the bioassay program would have asked you to enter dates to bracket both the period for the routine measurements and the period for which the intake is to be evaluated. For example, if you had selected the bioassay records for SSN CHRONICI131 from the RBDTEST data base, and had named the DAT file DEMOCIOD.DAT, the screen display would have been as follows (with user responses shown in italics):

RBDCOMP Ver. 4.1 07-01-92

Determination of Intake and Dose Using Bioassay Records

Developed for the US Army Materiel Command, Arlington, Virginia

Bioassay Records Filename: DEMOCIOD.DAT

Display Option: Batch (0) / Intake and Dose ([1]) / Ful1 (2): I

NUMBER OF RECORDS IN BIOASSAY FILE:

7

ROUTINE RECORDS FOUND BETWEEN DATES: 19890101 AND: 19900101

Enter Measurement Period - - Time Period for Considering Routine Measurements

Entering just the year [YYYY] followed by duration in years implies

Jan $1(00: 00)$ to Dec $31(24: 00)$ for the number of years specified.

Enter Beginning Measurement Date [YYYYMMDD] or [YYYY]: 1989

Enter Duration in Years: 1

Enter Intake Period - - Time Period for Intake and Dose Assessment

Entering just the year [YYYY] followed by duration in years implies

Jan $1(00: 00)$ to Dec 31 (24:00) for the number of years specified.

Enter Beginning Intake Date [YYYYMMDD] or [YYYY]: 1989

Enter Duration in Years: 1

Here the user has selected display option 1, the default response, to show intake and dose results to the screen. The user has entered a measurement window of 1989 (assumes 19890101) with a duration of 1 year and an intake window of 1989 (assumes 19890101) with a duration of 1 year. 


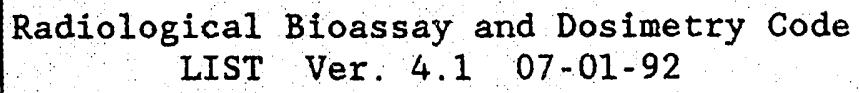

Select File to View
DEMOAIOD. LOG - RBDCOMP Listing
DEMOAIOD.REP * File does not exist *
DEMOAIOD.SUM * File does not exist *
DEMOAIOD.DAT - Bioassay Records File
DEMOAIOD.DBS - Output Records File
DEMOAIOD.CED * File does not exist *
Delete all files
Select new RBD run
Quit to menu

Upon selecting a file, the utility program BROWSE allows you to page through the file. To quit BROWSE press <Esc $>$.

Select and browse the DEMOAIOD.LOG file. Browse other files. Note that some of the files (those with extensions REP, SUM, and CED) do not exist. They are created when the REPORT program is run. From within BROWSE, press $<E s c>$ four times to return to the RBD main menu.

The example LOG file, DEMOAIOD.LOG, can be routed to a printer by selecting Print RBD Work Files from the RBD main menu. This menu item works exactly like the List RBD Work Files item described above, except that the file is routed to the printer instead of the screen. The selected file is printed using the DOS PRINT utility. If the DOS PRINT utility has not been installed, DOS will issue a prompt to which you simply press <Enter>. For the default parameters of the PRINT utility, PRINT will be working $20 \%$ of the time and the RBD software will be working $80 \%$ of the time. You may want to set the PRINT parameters to optimize performance. After viewing and/or printing all the files for a given example, the work files may be deleted by selecting menu item Delete all files. RBD work files can be archived using the FILMAN utility.

If a printer is attached to your computer, select RBD main menu item Print RBD Work Files. Select RBD Work Files and select the DEMOAIOD run. Print the file DEMOAIOD.LOG. Press <Enter > if prompted for Name of list device [PRN]. Return to the RBD main menu by pressing $<$ Esc $>$ three times. 


\subsection{LESSON 5. IMPORTING RECORDS INTO A BIOASSAY DATA BASE USING AUTOLOAD}

The AUTOLOAD utility allows one to load bioassay records, in RBD format, into a bioassay data base. A user who creates or alters bioassay records using an text editor can easily enter them into a bioassay data base using AUTOLOAD. To do so, you select Input Data Base - RBDBASE from the RBD main menu and then either select an existing data base or create a new data base. Then you select Run AUTOLOAD Utility from the RBDBASE menu.

Select Input Data Base - RBDBASE from the RBD main menu. Select Form New Data Base and type NUREG to create a new data base, RBDNUREG. Select the RBDNUREG data base. Finally, select Run AUTOLOAD Utility from the RBDBASE menu.

A list of bioassay records files (DAT files) appears. You select the one that you wish to load into the data base. More than one DAT file may be selected. When finished select Process Files, which is the last menu item, to load the selected file(s) into the data base. For each DAT file, you will be asked to confirm that the records from the file should be added to the data base. You respond Yes if this is the case, otherwise you respond No.

Select the file NUREG.DAT. Notice that the selected file is marked with arrows. Finally, select Process Files to load the bioassay records into the RBDNUREG data base. When asked if the DAT file should be added to the data base, respond Yes.

The data in the file NUREG.DAT are from NUREG/CR-4884 (ref. 8).

Browse the data base by selecting the RBDBASE menu item Browse RBDNUREG Data Base (see Lesson 3). Press <Esc > to return to the RBDBASE menu.

Extract the records for the SSN ACUTEU238A into a bioassay records file, $D E M O A U A . D A T$, and compute the intake and dose as described in Lesson 4. Quit the RBDBASE menu and select Bioassay Program - RBDCOMP from the RBD main menu to compute intake and dose for this example. Use display option 1 and enter the start of intake window as 1989 with the duration of 1 year.

The estimated intake and committed dose for the U-238 incident records selected from the $R B D N U R E G$ data base are shown on the next page. 


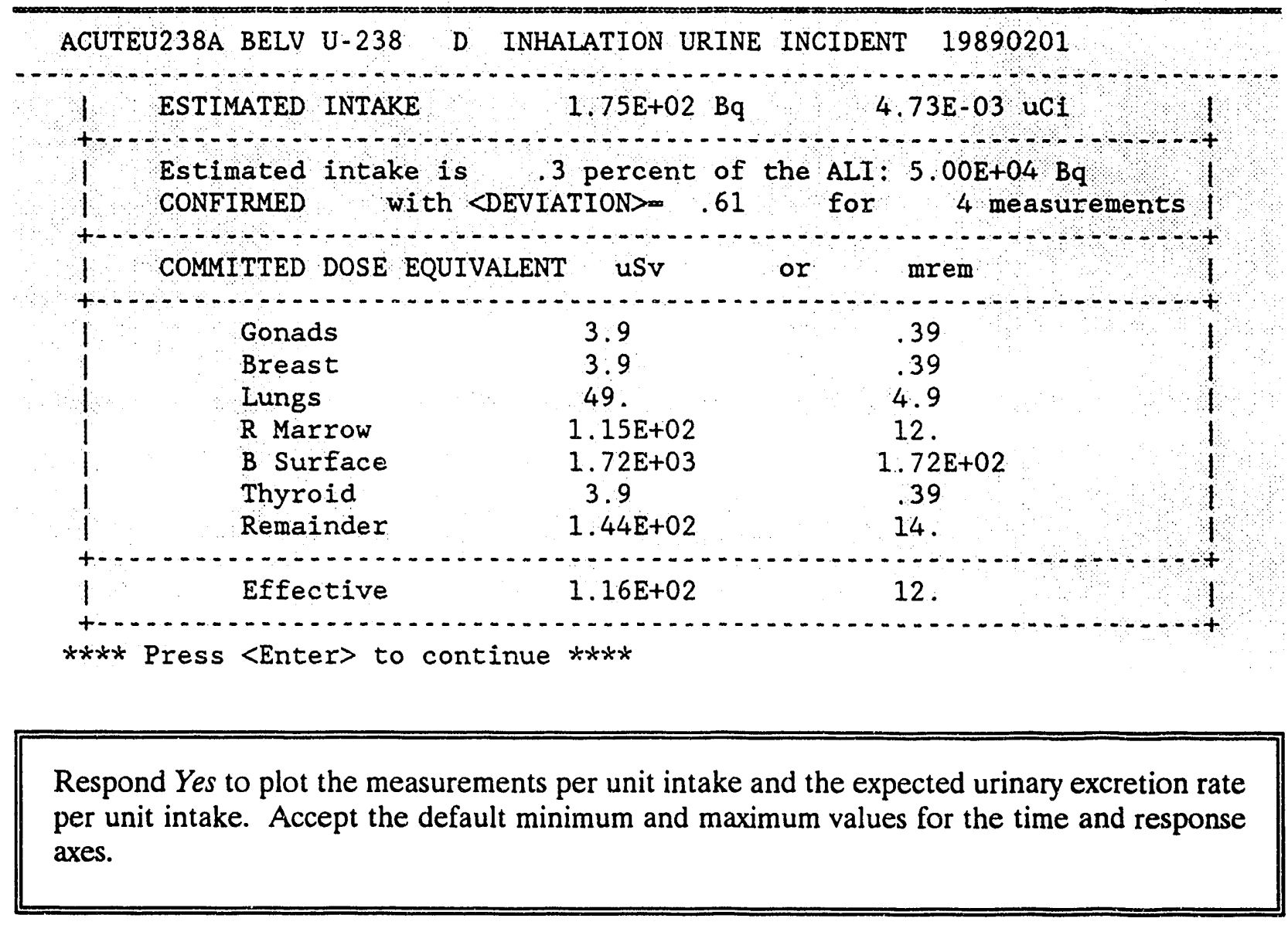

The plot is shown in Fig. 6 . The results of this computation will be entered into the output data base OUTNUREG in the next lesson.

Press $<$ Esc $>$ to exit the plot. 


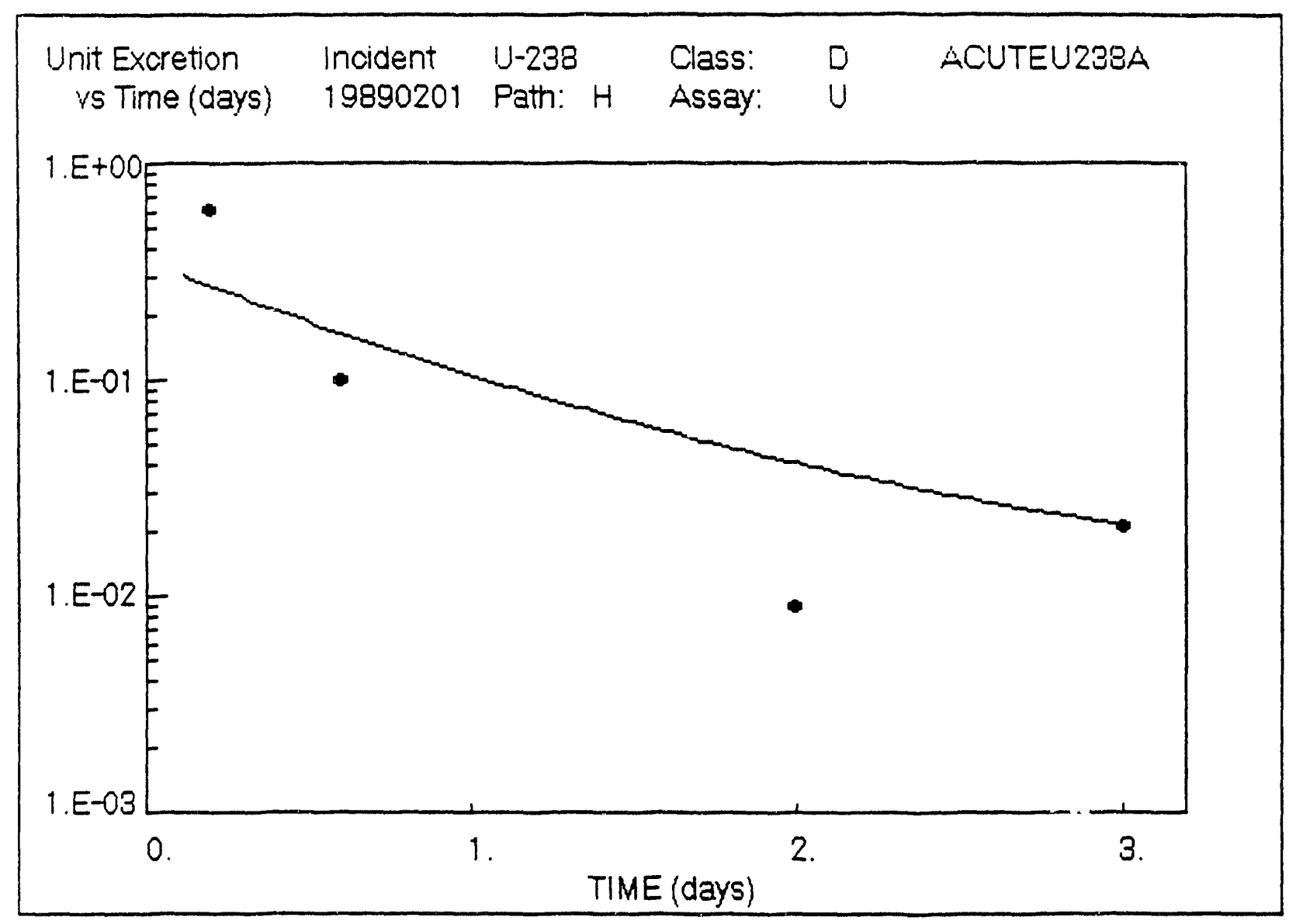

Fig. 6. Observed and expected urinary excretion for acute exposure to ${ }^{238} U$. 


\subsection{LESSON 6. IMPORTING RBDCOMP OUTPUT INTO AN OUTPUT DATA BASE}

After selecting a set of bioassay records and computing the intake and dose using RBDCOMP, the results of the computation, the output records file with extension DBS, can be placed into an output data base using the program RBDIMPOR. Note that the output data base wa; formed when the bioassay data base was created. By convention, the first three characters of the output data base name will be OUT. For example, OUTNUREG is the output data base corresponding to $R B D N U R E G$. To load the output records into an output data base, you select Output Data Base RBDIMPOR from the RBD main menu. You then select the output data base. To import the file into the data base, you select the menu item Import DBS File into OUTNUREG Data Base from the RBDIMPOR menu.

Select Output Data Base - RBDIMPOR from the RBD main menu and select the output data base OUTNUREG. From the RBDIMPOR menu, select Import DBS File into OUTNUREG

Data Base, as shown below.

\begin{tabular}{l} 
RBDIMPOR Ver. 4.1 \\
RBDCOMP Output File $\rightarrow$ Output Data Base \\
\hline Import DBS File Into OUTNUREG Data Base \\
BROWSE OUTNUREG by Calculation Date. \\
BROWSE OUTNUREG by SSN \& Nuclide. \\
Show RBDNUREG Data That Produced Given Result. \\
Quit RBDIMPOR.
\end{tabular}

Data bases for this session are OUTNUREG and RBDNUREG.

Import DBS file from RBDCOMP into OUTNUREG output data base. RBDIMPOR MENU

A list of output records files with extension DBS then appears, from which you select one to enter into the output data base. Select the desired DBS file to load into the OUTNUREG output data base. If the bioassay records came from a different data base, you will be asked if you wish to load the output records into the selected output data base. The output data base can then be browsed as described in the following lesson.

Select the DEMOAUA.DBS file to load into the OUTNUREG output data base. Exit the RBDIMPOR menu by selecting Quit RBDDMPOR (or pressing Q). 


\subsection{LESSON 7. BROWSING RECORDS IN AN OUTPUT DATA BASE}

To browse an output data base, you select Output Data Base - RBDIMPOR from the RBD main menu and select an output data base to browse. You will browse the output records contained in the output data base RBDTEST shipped with the RBD software.

Select Output Data Base - RBDIMPOR from the RBD main menu as shown below. Select the RBDTEST output data base. Finally, select Browse OUTTEST by SSN \& Nuclide.

\begin{tabular}{l} 
RBDIMPOR Ver. 4.1 \\
RBDCOMP Output File $\rightarrow$ Output Data Base \\
\hline Import DBS File into OUTTEST Data Base \\
BROWSE OUTTEST by Calculation Date \\
BROWSE OUTTEST by SSN \& Nuclide \\
Show RBDTEST Data That Produced Given Result \\
Quit RBDIMPOR
\end{tabular}

Data bases for this session are OUTTEST and RBDTEST.

Examine OUTTEST data base by SSN \& Nuclide.

\section{RBDIMPOR MENU}

Browsing by Calculation Date sorts the output records by date of calculation. Browsing by SSN and Nuclide sorts the output records by social security number first and then, within social security number, by nuclide. You should see the following screen after selecting BROWSE OUTTEST by SSN \& Nuclide: 


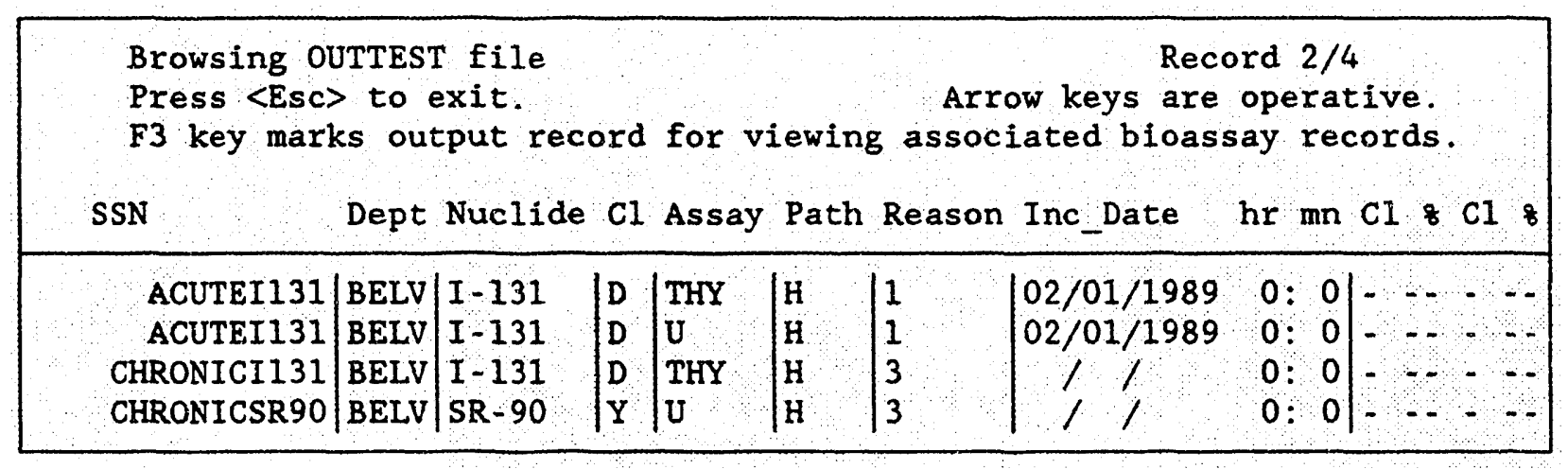

Use the arrow key to examine the records. Press $<\mathrm{Ctrl}><$ Home $>$ to return to the start of the records.

While browsing the output data base, you can determine which records were used from the bioassay data base to compute a given result. In the output data base, you move the cursor to the desired output result, and you press the $\langle F 3\rangle$ key. You should then exit the browse feature by pressing <Esc > and select the RBDIMPOR menu item Show RBDTEST Data That Produced Given Result. You can then browse the bioassay records in the bioassay data base which were used to compute the output result selected in the output data base.

Move the cursor to the first result in the OUTTEST data base and press the $<$ F3 $>$ key. Exit the browse feature by pressing <Esc> and select the RBDIMPOR menu item Show RBDTEST Data That Produced Given Result. You should see the thyroid count records for the I-131 incident from the RBDTEST bioassay data base as shown in the third lesson. When finished, press <Esc > to exit the bioassay data base and return to the RBD main menu by selecting Quit RBDIMPOR or pressing <Esc> twice.

If you h've imported output records resulting from a bioassay data base other than RBDTEST into OUTTEST, you wili get the message No RBDCOMP input records for this OUTTEST record when using this feature.

Pressing the heip key (the $<F 1>$ key) while browsing the output data base will display a table of codes used in the records.

Practice by browsing the output data base OUTNUREG created in the previous lesson. 


\subsection{LESSON 8. ENTERING EXPOSURE HISTORY RECORDS INTO A BIOASSAY DATA BASE, EXTRACTING RECORDS, AND COMPUTING INTAKE AND DOSE.}

In this lesson you will add exposure history records to the bioassay data base RBDNEW created in Lesson 2. To add exposure history records, you select Input Data Base - RBDBASE from the RBD main menu. After selecting the bioassay data base, you select the Enter Exposure History menu item. This menu item is similar to the item Add Data to Data Base, except that you enter exposure history information instead of bioassay measurement data. You can enter a start code (AAA) for start of chronic exposure and/or an end code (ZZZ) for end of chronic exposure to the nuclide for the selected individual. After entering the exposure history code, you enter the date and time of the start and/or end of the exposure period. The exposure is considered to be ongoing if no stop code is entered. All dates are entered in the form MM/DD/YYYY. Only the last two digits of the year need be entered. You cannot enter a start or end date beyond the current date obtained from your computer. If a date that is out of range is entered, an error message is printed and you must enter a correct date.

Select Input Data Base - RBDBASE from the RBD main menu and select the RBDNEW data base created in Lesson 2. From the RBDBASE menu, select Enter Exposure History.

To add an exposure history record for an existing subject in the data base, you select the individual's name from the list at right, shown below.

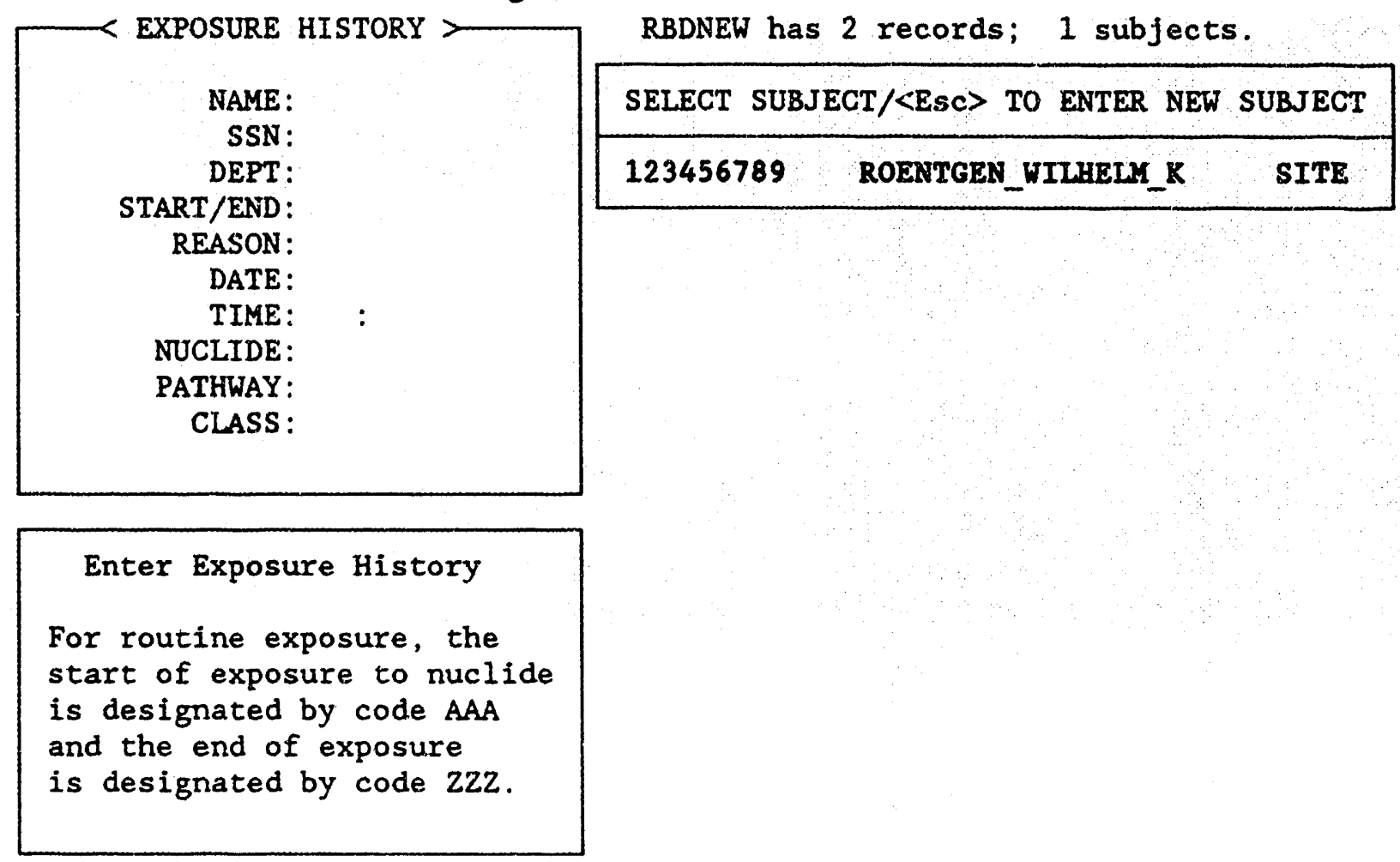

Select the individual ROENTGEN (SSN: 123456789). 
If history records were to be added to the data base for a new subject, you would press the <Esc> key and enter the individual's name (up to 25 characters) in the name field. After selecting the individual ROENTGEN, you select the exposure code AAA for start of exposure to Cs-137.

Select an exposure start code (AAA) for start of exposure to Cs-137.

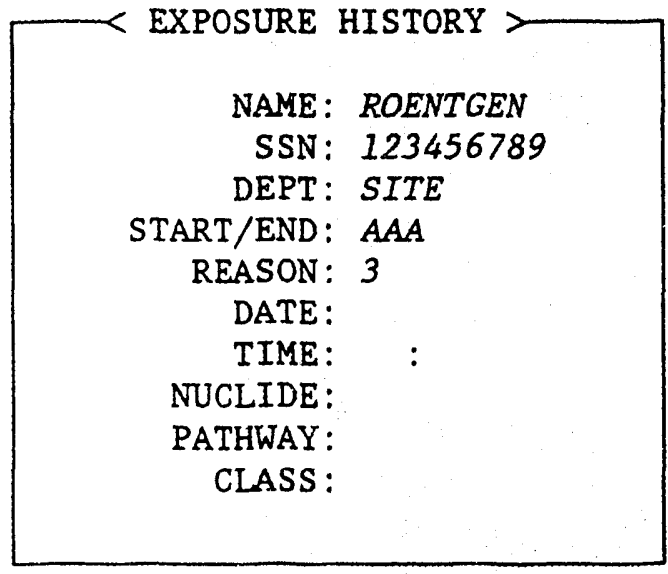

Exposure Codes

AAA Exposure Start Code

ZZZ Exposure End Code

Enter Exposure History

For routine exposure, the

start of exposure to nuclide

is designated by code AAA

and the end of exposure

is designated by code $\mathrm{ZZZ}$.

On selecting an exposure code, the program automatically enters a reason code of 3 , because exposure history records are associated only with routine (chronic) measurements. You then enter the date and time of start of exposure, the nuclide, pathway, and class. As with bioassay records, a comment field is attached to the end of each of the exposure history records.

Enter a date of 07/01/1989 and a time of 00:00. Select CS-137 as the nuclide and inhalation $(H)$ as the pathway. Select $D$ as the inhalation class and enter comments, as shown on the following page. 


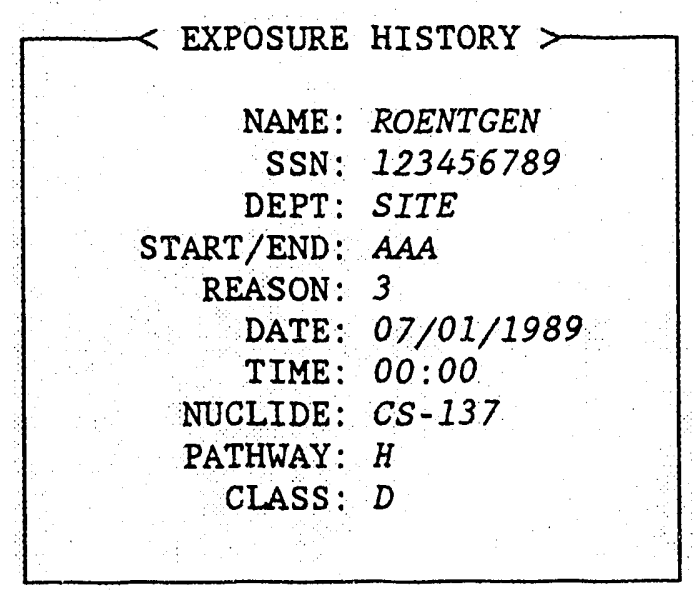

Add any comments: Began working in biology laboratory.

After any corrections are made, the exposure history record is added to the $R B D N E W$ data base. Another exposure history record can then be entered for the same individual (1) or for another individual (2).

Add an exposure end code for the above case. Select (1) to add another exposure history record for the same individual. Then select exposure end code (ZZZ). Change the date to $12 / 31 / 1989$ and the time to $24: 00$. Select Cs-137 as the nuclide, inhalation (H) as the pathway, and inhalation class D. Again add comments as shown.

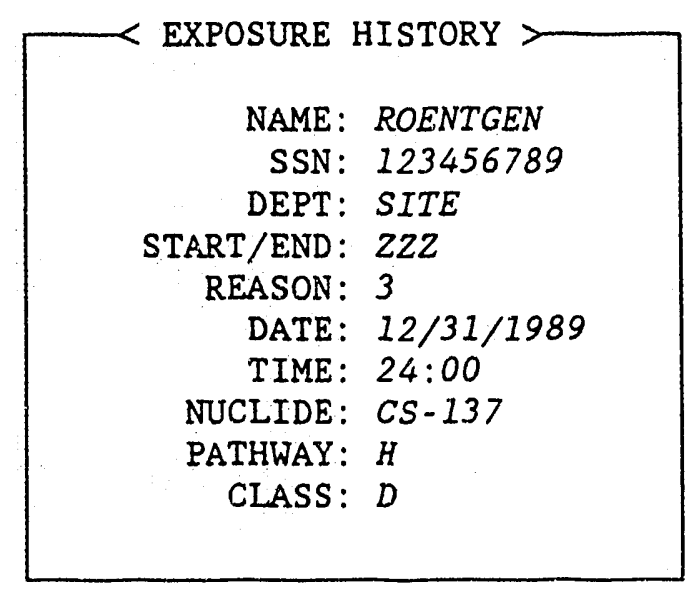

Add any comments: Transferred to physics laboratory.

Make any corrections needed and exit the exposure history form by typing 3 followed by $<$ Enter $>$. 
The aerodynamic median aerosol diameter (AMAD) parameter is not specified on exposure history records. This means that one cannot distinguish exposure history for specific particle size, only for nuclide and chemical form (inhalation or ingestion class).

Return to the RBDBASE menu and extract all the records from the $R B D N E W$ data base. Name the bioassay records file DEMO_CS. This file (DEMO_CS.DAT), which will be input to the bioassay program, will contain records for both the acute and chronic exposure cases involving Cs-137. Then return to the RBD main menu and select Bioassay Program RBDCOMP. Select the file DEMO_CS.DAT to compute the intake and dose. Use default display option 1 and enter measurement and intake windows as 1989 (assumes 19890101) with a duration of 1 year.

The listings to the screen by the RBDCOMP program for this example, DEMO_CS, are shown on the following pages. The plots (Figs. 7 and 8 ) of the measurements per unit intake and the unit retention function for the acute and chronic exposure to Cs-137 are shown following the listings.

After the computations are completed, return to the RBD main menu and select Output Data Base - RBDIMPOR to import the output records file (DEMO_CS.DBS) into the OUTNEW data base. Examine, using the RBDIMPOR browse feature, the contents of the output data base. Use the arrow keys to move within the output records. Note that the intake and committed doses match those shown in the output listings on the following pages. Press $<$ Esc > to exit the browse feature and select Quit RBDIMPOR to return to the RBD main menu. 
RBDCOMP Ver. 4.1 07-01-92

COMPUTATION DATE: $7 / 1 / 1992$

Determination of Intake and Dose Using Bioassay Records

Developed for the US Army Materiel Command, Arlington, Virginia

Bioassay Records Filename: DEMO_CS.DAT

Display Option: Batch (0) / Intake and Dose ([1]) / Full (2): 1

NUMBER OF RECORDS IN BIOASSAY FILE:

ROUTINE RECORDS FOUND BETWEEN DATES: 19890701 - 19891231

OCCURRENCE OF INCIDENT BETWEEN DATES: 19890101 - 19890101

INCIDENT RECORDS FOUND BETWEEN DATES : 19891231 - 19891231

Enter Measurement Period -. Time Period for Considering Routine iseasurements: Entering just the year [YYYY] followed by duration in years implies Jan $1(00: 00)$ to Dec $31(24: 00)$ for the number of years specified.

Enter Beginning Measurement Date [YYYYMMD] or [YYYY]: 1989

Enter Duration in Years:

Enter Intake Period - - Time Period for Intake and Dose Assessment:

Entering just the year [YYYY] followed by duration in years implies

Jan $1(00: 00)$ to Dec $31(24: 00)$ for the number of years specified.

Enter Beginning Intake Date [YYYYMMDD] or [YYYY]: 1989

Enter Duration in Years:

1

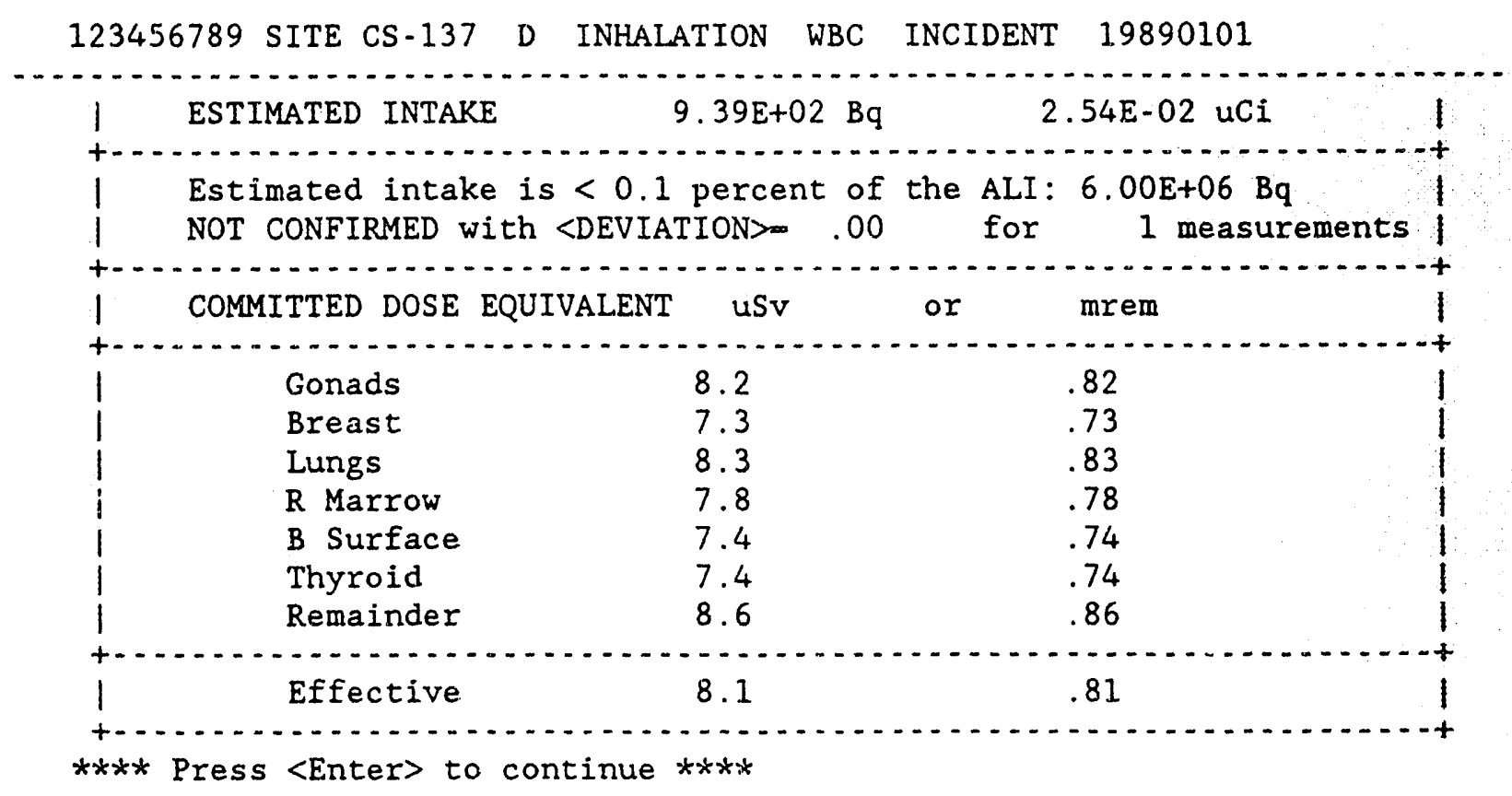




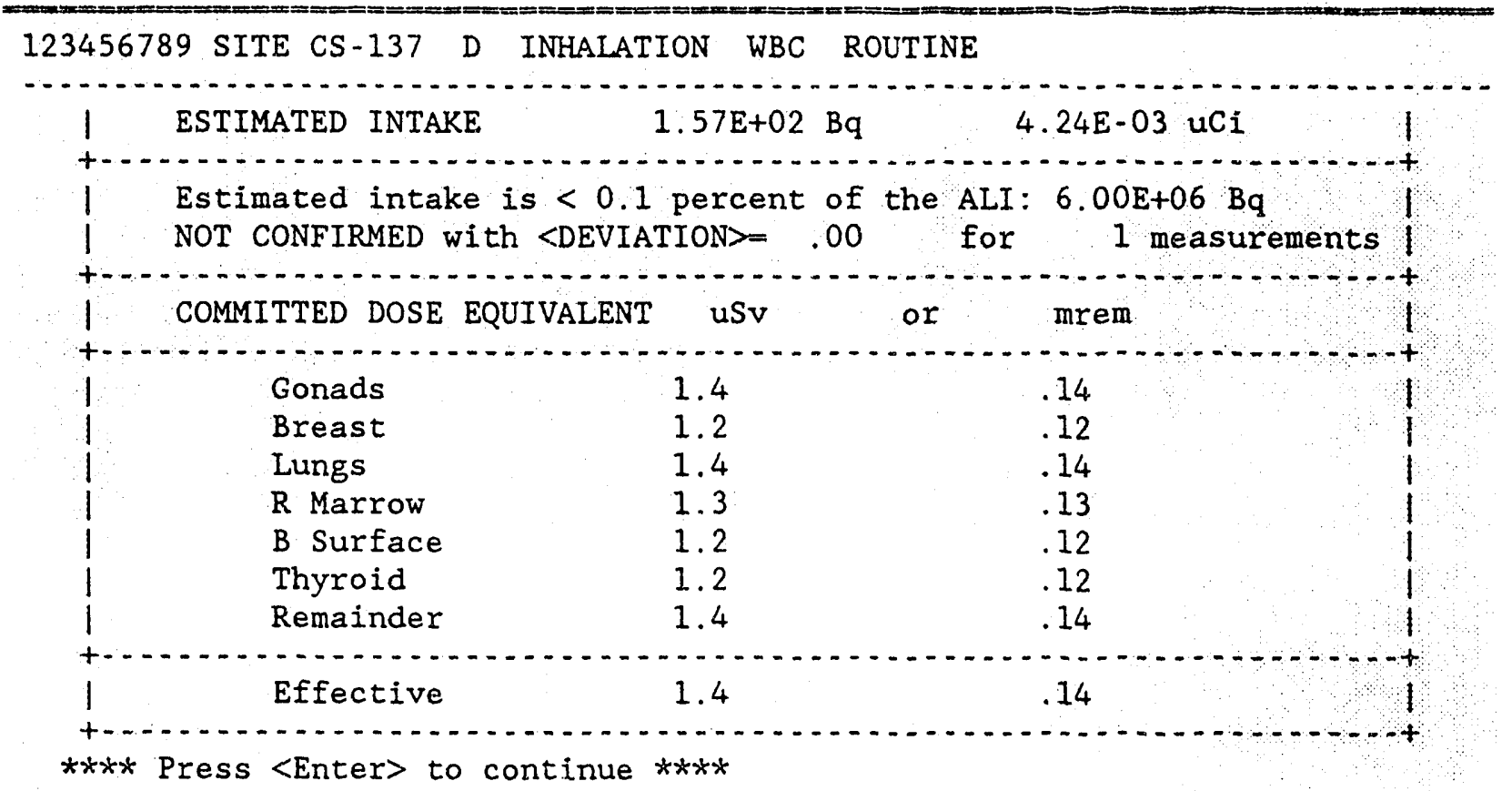




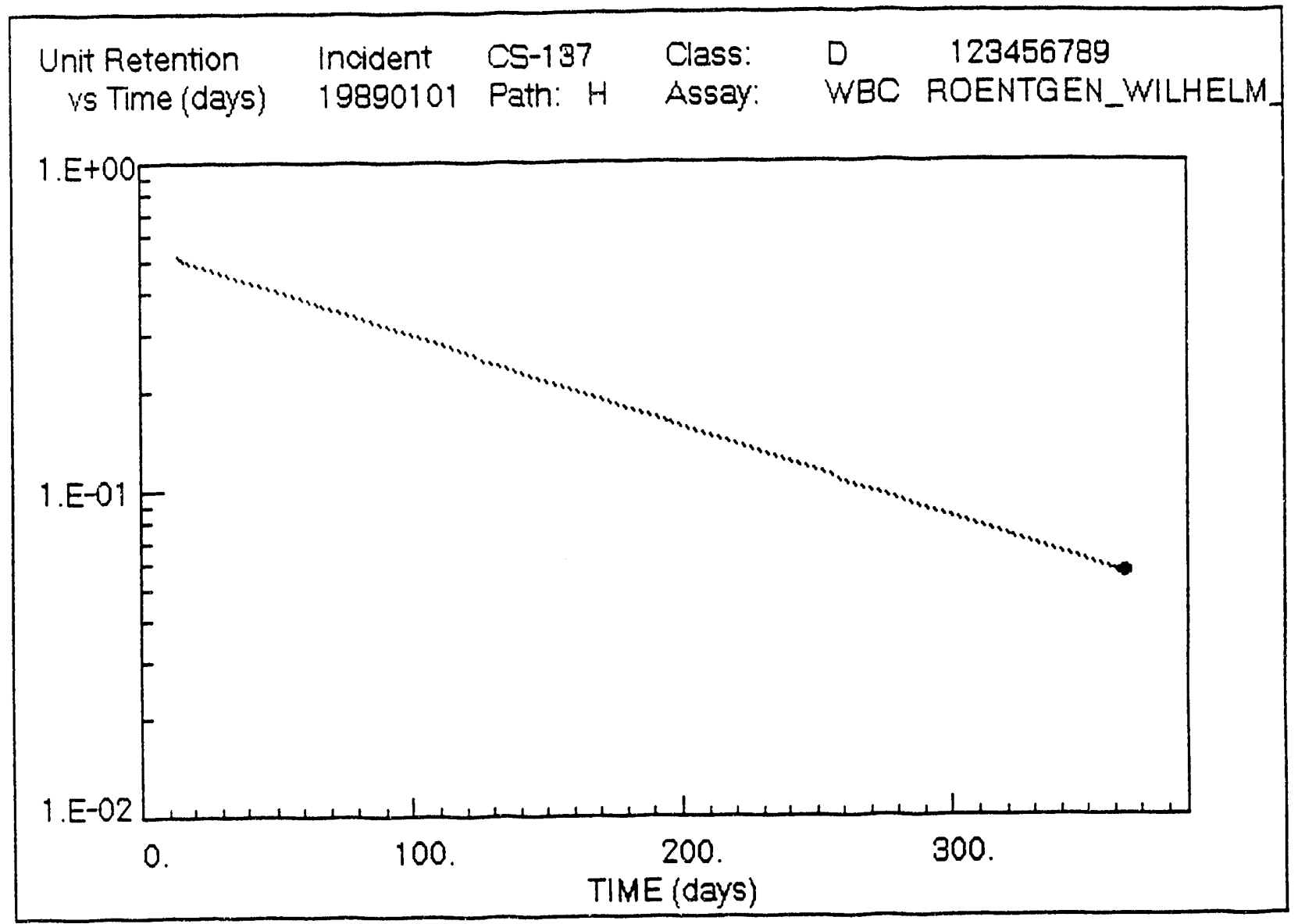

Fig. 7. Observed and expected whole body retention for acute exposure to ${ }^{137} \mathrm{Cs}$. 


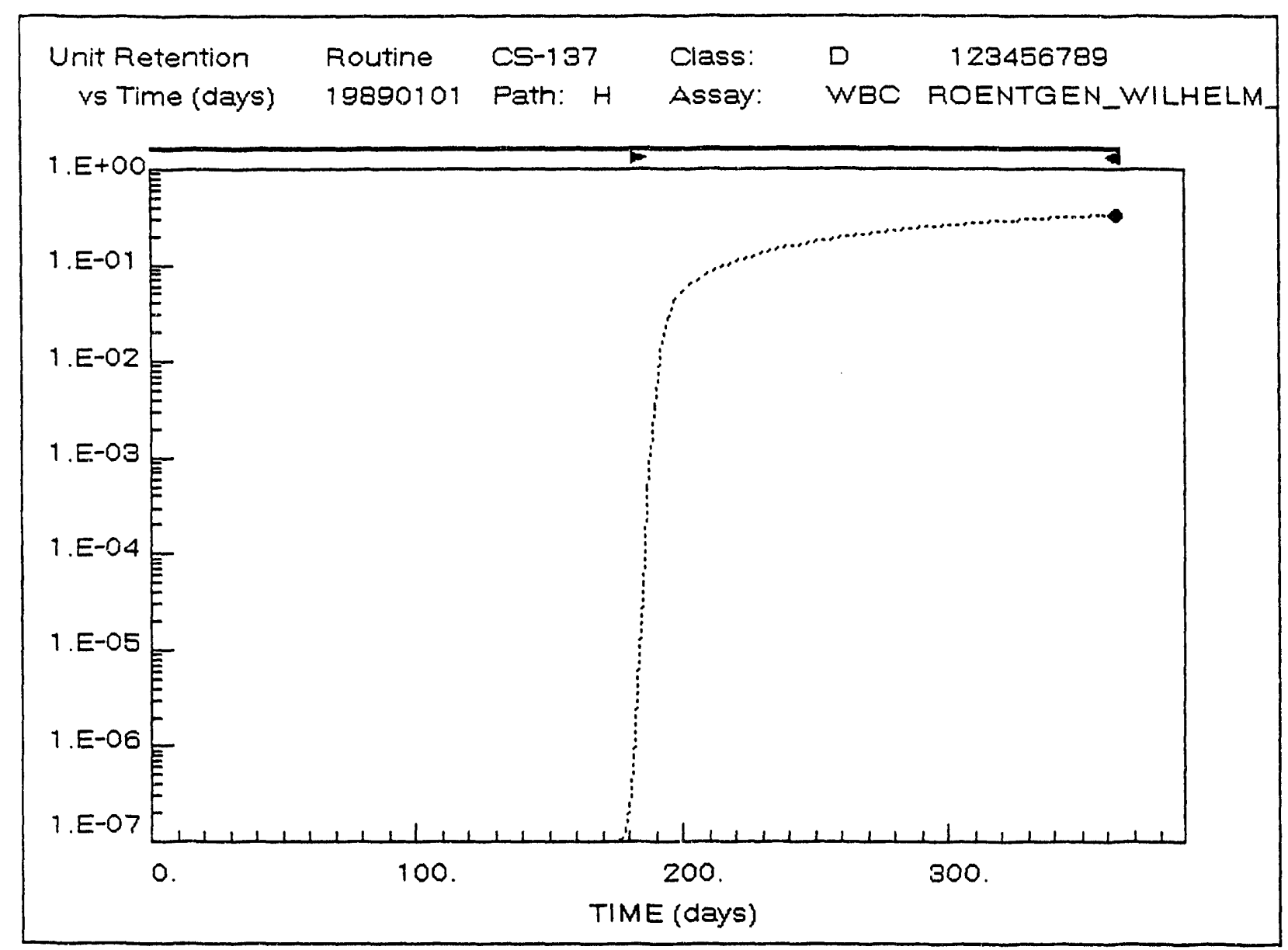

Fig. 8. Observed and expected whole body retention for chronic exposure to ${ }^{137} \mathrm{Cs}$. 


\subsection{LESSON 9. USING THE MEASUREMENT AND INTAKE WINDOWS IN COMPUTING INTAKE AND DOSE}

The bioassay program (RBDCOMP) asks you for starting and ending dates, in the form YYYYMMDD, for bracketing routine measurements. This time period is referred to as the measurement window. You can also enter the year in the form YYYY followed by a duration in years for considering measurements over an integral number of years.

In addition, RBDCOMP asks for starting and ending dates, in the form YYYYMMDD, to define the period over which the intake will be computed. This time period is referred to as the intake window. You can also enter the year in the form YYYY followed by a duration in years for considering measurements over an integral number of years. In this lesson you will learn how to use the measurement and intake windows for a routine bioassay.

Select Input Data Base - RBDBASE from the RBD main menu, and select the RBDTEST data base. Choose Select Input for RBDCOMP from the RBDBASE menu, and select SINGLE SSN. Use the menu bar to select CHRONICSR90 HAFIN OTTO BELV. Enter DEMOSR90 as the bioassay records (DAT) filename. Press $\langle\mathrm{Esc}\rangle$ twice to return to the RBD main menu.

Select Bioassay Program - RBDCOMP from RBD main menu to compute the intake and dose as described in Lesson 4. Select DEMOSR90.DAT as the input. Select Execute the RBDCOMP Program. Use default display option 1. Since this is a routine case (chiconic exposure), you will be asked for the measurement window - the time period for acceptirng routine measurements. Enter the start of measurement period as 1989 (assumed to be 19890101) with the duration of 2 years.

Ar this point you are asked for the intake window - the period of time for computing the intake. This example contains a period of known exposure to $\mathrm{Sr}-90$, the year 1989, which is indicated using exposure history records. This period of exposure is followed by a period when the individual was known not to be exposed to Sr-90, the year 1990. Post-exposure bioassay measurements were made during 1990. In this lesson you will compute intake and dose for three different intake windows: (1) 1989 with duration of 1 year, (2) 1990 with duration of 1 year, and (3) 1989 with duration of 2 years. The intake computed in 1990 should be zero, and the intake computed over the 2-year period (1989 and 1990) should be the same as the intake computed over the year 1989.

For the first run, enter the start of the intake period as 1989 (assumed to be 19890101) with a duration of 1 year.

You should see the following results for intake and dose. 


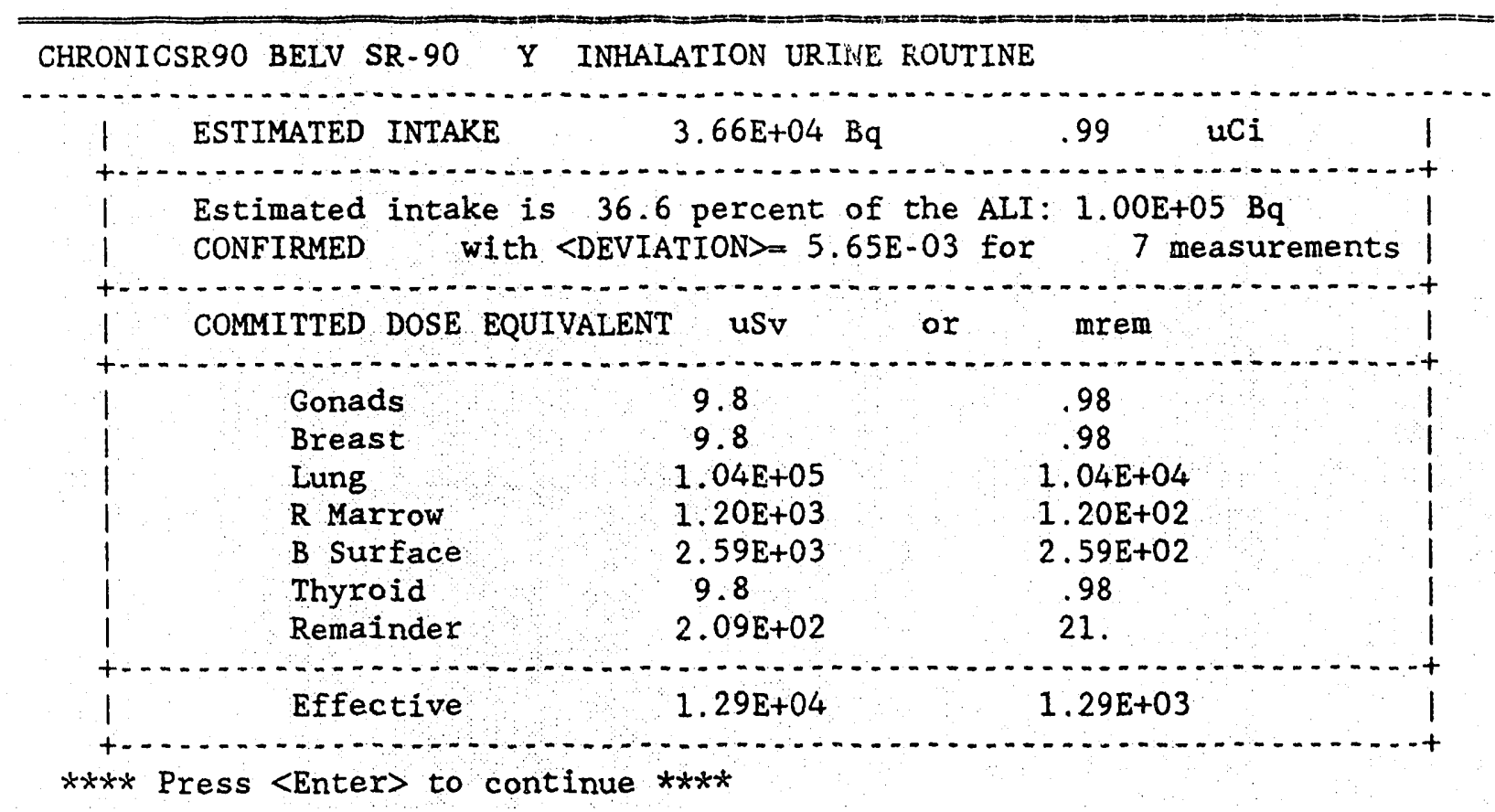

Respond Yes to Plot the measurements per unit intake and unit response function? Accept the default minimum and maximum values for the time and response axes.

The resulting plot is shown in Fig. 9. Note that the bar across the top of the graph, which indicates the period of intake, extends only over the first year. The start and end of exposure to Sr-90 are indicated using arrows underneath the intake bar. The post-exposure measurements are indicated with hollow circles, and the measurements during the exposure period are indicated with solid circles.

Press $<$ Esc $>$ to exit the plot.

Again select Bioassay Program - RBDCOMP from RBD main menu, and select the file DEMOSR90.DAT for input. Select Execute the RBDCOMP Program. Again use screen display option 1 and enter the measurement period as 1989 with a duration of 2 years. For this second run, enter the intake period as 1990 with a duration of 1 year.

You should see that the intake is zero as shown below. Since the intake is zero, for this case, no plot of measurements per unit intake is done.

CHRONICSR90 BELV SR $90 \quad$ Y INHALATION URINE ROUTINE
1 $* * *$
$\quad$ INTAKE IS ZERO FOR ASSAY




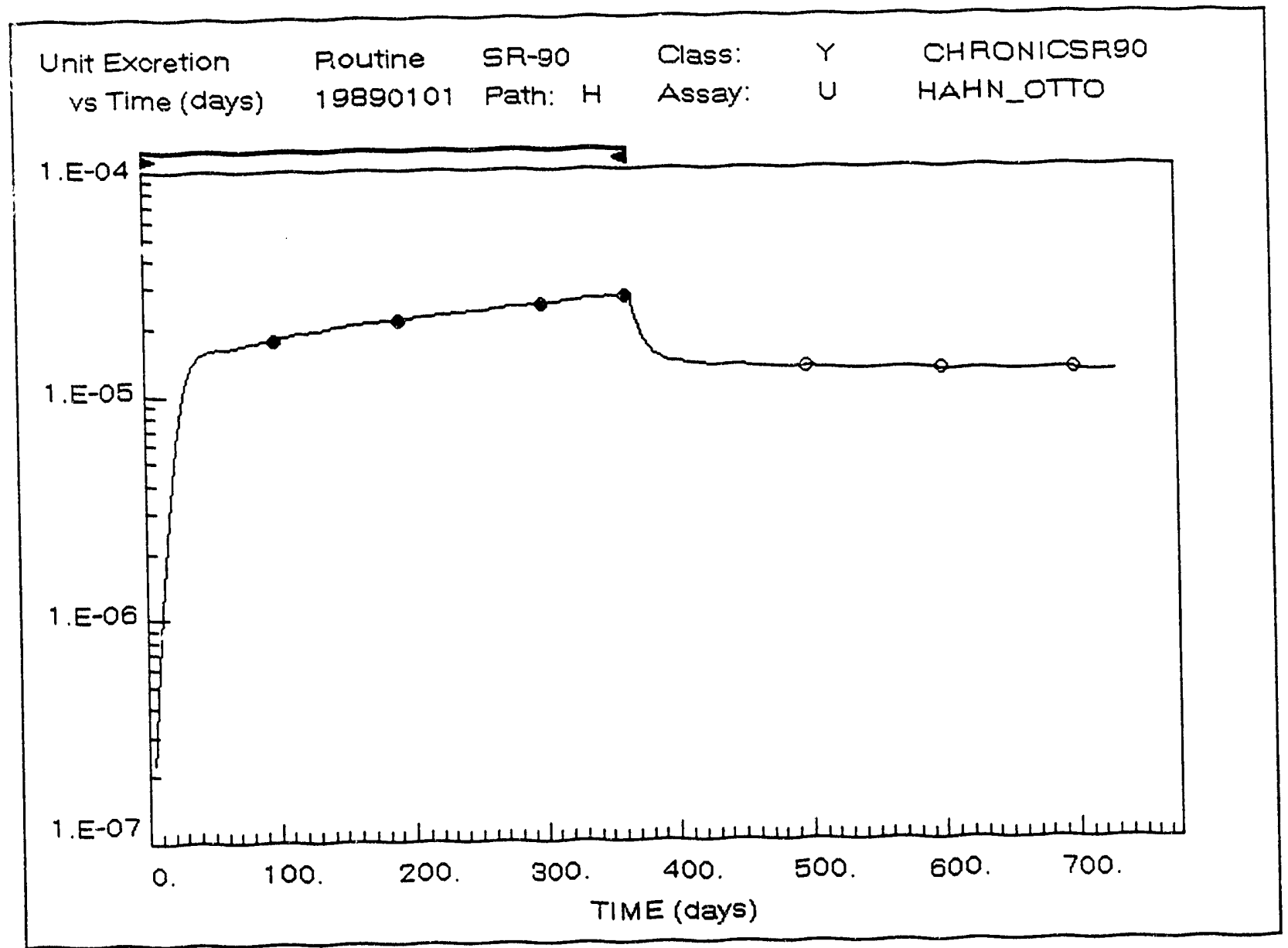

Fig. 9. Observed and expected urinary excretion for chronic exposure to ${ }^{90} \mathrm{Sr}$. 
Again select Bioassay Program - RBDCOMP from RBD main menu and select the file DEMOSR90.DAT for input. Select Execute the RBDCOMP program. Again use display option 1. For this third run, enter identical measurement and intake windows. Enter the measurement and intake period as 1989 with a durat: ${ }^{-m}$ of 2 years.

You should see the same results as in the first example above.

Respond Yes to Plot measurements per unit intake and unit response function? Accept the default minimum and maximum values for the time and response axes.

The resulting plot is like that in Fig. 9 except that the intake bar, which indicates the period of intake, extends over the entire 2 years. The start and end of exposure to Sr-90 are indicated using arrows underneath the intake bar. The post-exposure measurements are indicated with hollow circles, and the measurements during the exposure period are indicated with solid circles.

Press <Esc $>$ to exit the plot and return to the RBD main menu. 


\subsection{LESSON 10. GENERATING A COMMITTED DOSE REPORT USING REPORT}

A committed dose report, showing the committed dose to each individual, will be generated automatically when RBDCOMP is run if the REPORT switch in SITE.DFT is set to true (T). Alternatively, if the REPORT switch is set to false (F), a committed dose report can be generated from an existing DBS file by selecting Generate REPORT from the RBD main menu. To set the REPORT switch, you select Modify SITE.DFT - MODSITE from the RBD main menu and toggle item 16. In Lesson 1, you set the REPORT switch to false.

Three output files are produced by the REPORT program: an output listing (with extension REP), a summary listing (with extension SUM) of committed organ and effective dose equivalents by SSN, and an output file (with extension CED) containing committed effective dose equivalent for each individual. The format of the CED file is specified in Appendix C.

In this lesson you will learn to generate a committed dose report and summary for the acute exposure to I-131 described in Lesson 4.

Select Generate REPORT from the RBD main menu and select the file DEMOAIOD.DBS.

Select Execute REPORT Program (or press E) to run the program.

The REPORT program header should appear as shown below.

REPORT Ver. 4.1 07-01-92

COMPUTATION DATE: $7 / 1 / 1992$

Determination of Intake and Dose Using Bioassay Records

Developed for the US Army Materiel Command, Arlington, Virginia

DBS Filename: DEMOAIOD. DBS

Display Option: Batch (0) / Modify Weights ([1]) / Full (2): 1

The committed dose report is generated from the output records (DBS) file. As with RBDCOMP, there are three types of screen output: (0) Batch, which does not allow modification of assay weights; (1) Modify Weights, which does allow modification of assay weights if SITE.DFT switch has been set; and (2) Full, which gives weighted organ doses in addition to weighted committed effective dose. Display option 1 is the defau?.

Press < Enter > to seect the default display option $(1)$ and press < Enter $>$ again to clear the program header.

The output to the screen should appear as shown on the following page. 


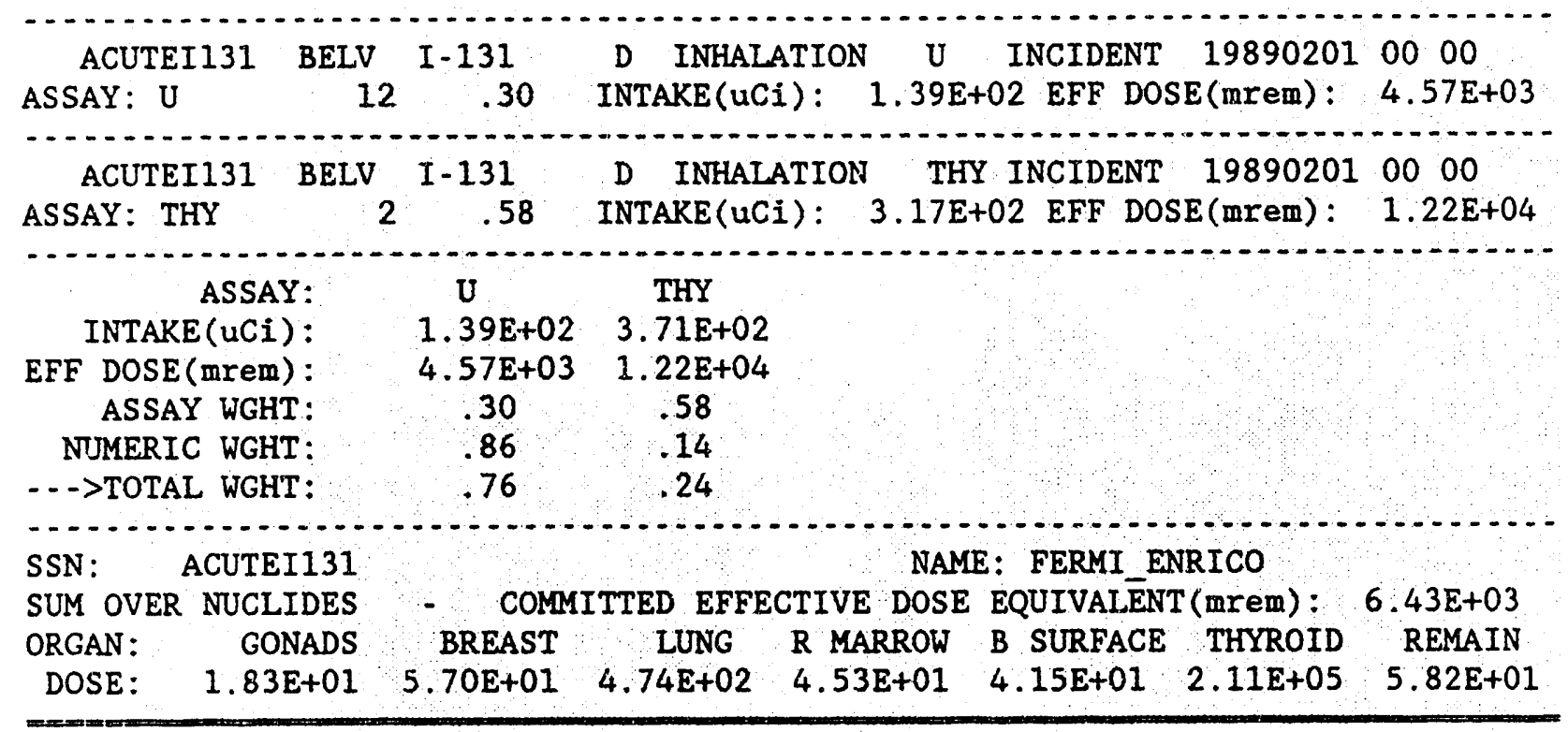

Note that this example has both urine and thyroid measurements. The total weighting factors are the product of the weight for each assay assigned in the nuclide file (ASSAY WGHT) and a numerical weight (NUMERIC WGHT) equal to the number of measurements for a given assay divided by the total number of measurements. The resulting weights are normalized so that their sum is 1 . In the above example, there were 14 measurements: 12 urine measurements and 2 thyroid measurements with numerical weights of 0.86 and 0.14 , respectively. The assay weights in the I- 131 nuclide file for urinalysis and thyroid count are 0.30 and 0.58 , respectively. The normalized total weights are 0.76 for urinalysis and 0.24 for the in vivo measurements of the thyroid activity. Using these weights, the estimated intake is $0.76 \times 139 \mu \mathrm{Ci}+0.24 \times 371 \mu \mathrm{Ci}$ or $195 \mu \mathrm{Ci}$, and the effective committed dose equivalent is $0.76 \times 4.57 \mathrm{rem}+0.24 \times 12.0 \mathrm{rem}$ or $6.43 \mathrm{rem}$.

You can override the total weights by setting the WGTMOD switch in SITE.DFT to true (T). To modify the total weights, respond Yes to the question MODIFY TOTAL WEIGHTS? and enter a total weight for each assay. The sum of the weights must be equal to 1 . The intake and dose computed using these weights will then be displayed. If you accept these total weights, respond Yes to the question ACCEPT TOTAL WEIGHTS? Respond No to alter the total weights. Once you have accepted the total weights, the RBD output records file (DBS file) will contain a -1 in the field which previously contained the number of bioassay records. In addition, the field that previously contained the assay weight now contains the total weight. Once accepted, the assay weighting factors cannot be altered except by recomputing the intake and dose using RBDCOMP.

Press <Enter $>$ to return to the RBD main menu.

This concludes the RBD software tutorial. You should explore other capabilities of the RBD software not covered in this tutorial, such as the RBD utilities SORTREC, FILMAN, LISTNUC, and PLOTNUC, which are discussed in Sect. 6. 


\section{DETAIIS OF OPERATION OF RBD PROGRAMS AND UTILITIES}

\subsection{INTRODUCTION}

The RBD software was designed to run interactively on an IBM-compatible personal computer ( 80286 or higher CPU) with $640 \mathrm{~K}$ of memory and a hard disk. Due to the present size of the RBDCOMP program (412K), you must be sure that sufficient memory is available to run the software. A math co-processor is not required, but it is recommended and will be used if present. The entire RBD software package requires just over 3.2 Megabytes of disk space at the time of installation. You should be certain that sufficient additional space is present for RBD working files.

Input to the bioassay program is obtained by extracting records from a bioassay data base. The format for the bioassay records (DAT) file is shown in Appendix A. The format is identical for in vivo and in vitro assays for all variables up to the incident time, as ihese variables are used in sorting the records. Beyond that the records differ only in the presence of the assay sample volume (or mass) and excretion rate for in vitro assay.

The REASON code indicates whether the record is an incident (1) or a routine (3) exposure. In vitro assays presently allowed in RBD are breath (BRE), urine (U), and feces (F). In vivo assays considered are whole body count (WBC), lung count (LUN), and thyroid count (THY). The pathways of exposure included in RBD are inhalation $(\mathrm{H})$, ingestion $(\mathrm{G})$, and wound (W). Committed doses are not computed for wound pathway; only the intake is computed.

The classes allowed for inhalation are day (D), week (W), year (Y), vapor (V) [e.g., tritiated water vapor], labeled organic compounds (L) [e.g., radiocarbons], and mixed class (M). If mixed class is chosen, you must also add a mixed-class identifier (0-9) making the class designation $\mathrm{MX}$, where $\mathrm{X}$ is a number between 0 and 9 . You must also supply the percentage of $D, W$ and $Y$ defining the mixture. The classes allowed for ingestion are very insoluble (V), insoluble (I) and soluble (S).

\subsection{DETAILS OF THE BIOASSAY PROGRAM (RBDCOMP)}

The bioassay program (RBDCOMP) computes intake and dose when given a sorted bioassay records file. RBDCOMP writes two output files. One is a listing (with extension LOG) of the computed intake and dose and includes a summary of the bioassay records. The results appear in the same order as the sorted bioassay records input (DAT) file. The second RBDCOMP output (with extension DBS) is used to import the intake and dose into an output data base. The format for the DBS file is shown in Appendix B.

The bioassay program first prints a header giving the version and date of the RBD program, the date of the computation, and the name of the bioassay data file. If you have previously computed intake and dose for an input file, the output files (LOG and DBS files) will already exist. In this case the bioassay program asks if you wish to replace these files. If you respond $\mathrm{No}$, the program asks for a new name for the output files.

You are then asked for the type of screen display. Batch (selection 0 ) is used for batch processing. Intake and Dose (selection 1, the default) gives intake and dose but no bioassay record information. Full (selection 2) gives bioassay records information in addition to intake and dose results. Selections 1 and 2 prompt you regarding graphical output, if the PLOT switch in the SITE.DFT file is set to 
true (T). See Appendix D for a listing of the SITE.DFT file.

If the bioassay program detects that there are routine records to be analyzed, the program will ask for a starting and ending date, in the form YYYYMMDD, for bracketing the period of routine measurements. This time period is referred to as the measurement window. You can also enter the year in the form YYYY followed by a duration in years for considering measurements over an integral number of years.

Finally, RBDCOMP asks for a starting and ending date, in the form YYYYMMDD, for the period over which the intake is to be computed. This time period is referred to as the intake window. You can also enter the year in the form YYYY followed by a duration in years for considering measurements over an integral number of years.

During execution, the bioassay program first produces a scratch file for controlling batch execution. It contains a line of information on each case (SSN, site, nuclide, class, and reason codes) and the number of records for each case. The program then reads site-specific information from the SITE.DFT file. The purpose of this file is to allow users to tailor the program to their needs. The SITE.DFT file, as shipped with RBD software, is listed in Appendix D. The SITE.DFT file contains the following information:

- A list of all nuclide data files existing when the software was shipped.

- The number of nuclides (with nuclide data files) expected at the site. Each name is followed by the starting record number for that nuclide in the binary, direct-access file NUCLIDE.BIN.

- A list of the nuclide files that are used at the site.

- Default value of AMAD $(\mu \mathrm{m})$.

- Value for urinary excretion (mL/day).

- Default value for the volume of urine per aliquot sample (mL/sample).

- Value for fecal excretion (g/day).

- Default value for the mass of fecal sample analyzed (g/sample).

- Value for breath excretion (L/day).

- Default value for the breath volume per sample ( $\mathrm{L} /$ sample).

- Default value for the number of days (or fraction of days) between the occurrence of an incident and the first measurement. This is used if incident date is missing from the record. 
- RMDALC - Ratio of MDA (minimum detectable activity) to LC (lower critical limit).

- NUMLC - Number of records above LC required to confirm intake.

The following two parameters control the unit retention/excretion plot:

- BREAK - The time (days) when the time axis of the plot breaks from linear to log.

- CUTOFF - The minimum value of the vertical axis in the unit retention/excretion plot.

The following toggle switches control operation of the bioassay program and the REPORT program. Setting a switch to true (T) turns on the option; setting the switch to false (F) turns off the option.

The switches control:

- Plotting of unit excretion/retention function.

- Generation of committed dose report.

- Modification of assay weighting factors for committed dose report.

- Creation of intake rate file for routine exposure.

- Debugging of code using trace.

- Beep when user response is required or "Press <Enter $>$ to continue" is displayed.

Lastly, you can specify

- Drive and path for scratch files used by RBDCOMP.

After reading the SITE.DFT file, the bioassay program reads records from the input data file as directed by the control file. The program then proceeds through this control file, doing the computations for a given social security number, department, nuclide, class, and reason code as listed. The bioassay program can process 200 records for this set of parameters, which is referred to as a case. For a given case, the program loops over all assays - breath assay (BRE), urinalysis (U), fecal assay (F), whole body count (WBC), and lung count (LUN). If in vivo measurements involve a systemic organ, for example thyroid measurement for radioiodine, the corresponding assay designation (in this case THY for thyroid) will also be in the above loop. Additional specific organ assays for other nuclides can be easily added in the future.

An illustration of the order of processing cases by RBDCOMP is shown in Fig 10. Consider routine and incident exposure for an individual with SSN 123456789 at department HEAD to 
nuclides U-235 (inhalation classes D and $\mathrm{Y}$ ) and U-238 (inhalation class Y). RBDCOMP loops in order through breath, urine, fecal assay, whole body count, and lung count. Note that the routine records are sorted before any of the incident records, and the incidents are sorted by incident date.

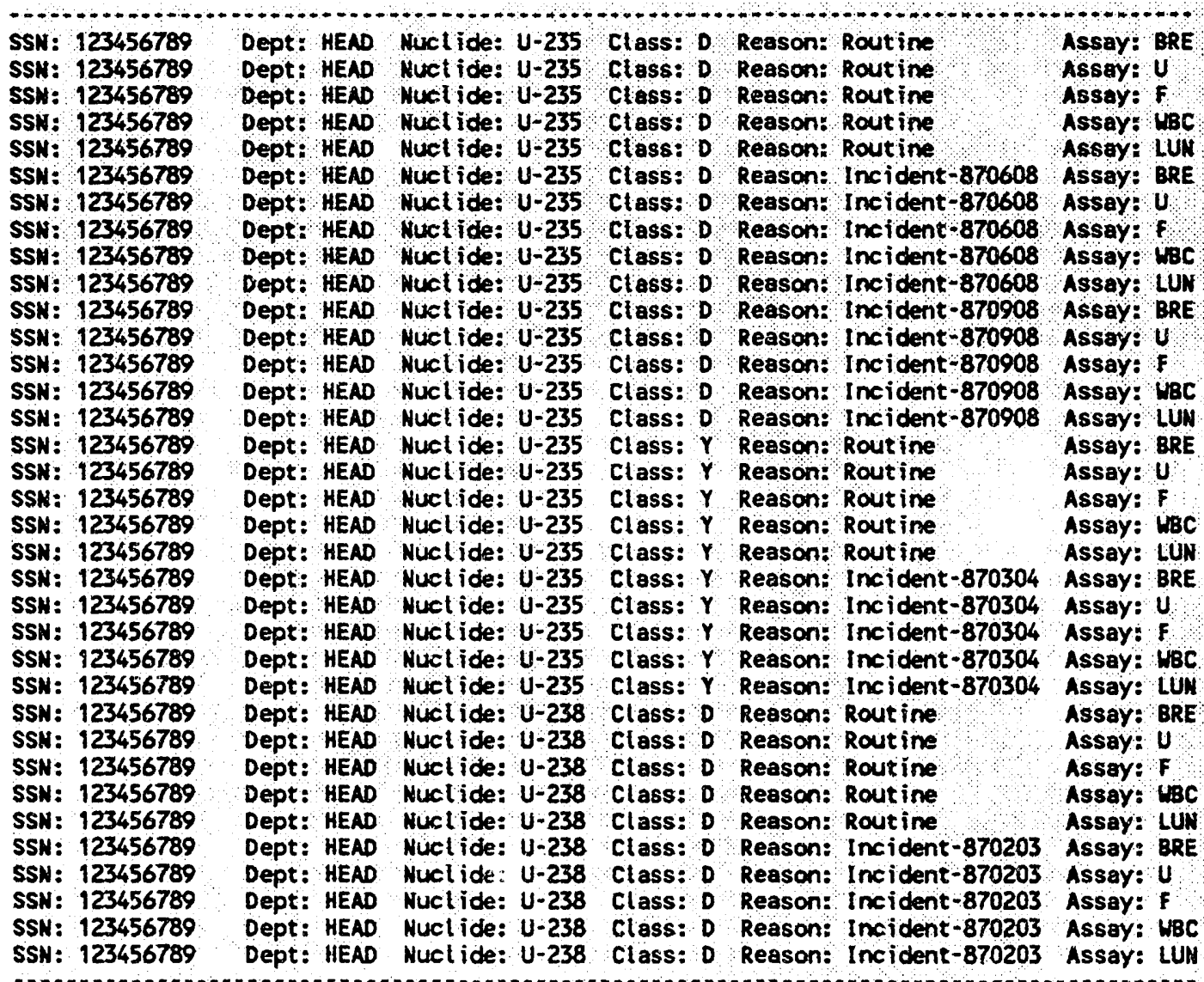

Fig. 10. Order of Processing Cases by RBDCOMP.

When RBDCOMP has finished with all the entries in the control file, the REPORT progaim will be executed if the REPORT switch in the SITE.DFT file has been set to true (T). Otherwise, when RBDCOMP finishes, you are returned to the RBD main menu. 


\subsection{ASSUMPTIONS MADE IN THE BIOASSAY PROGRAM}

The following assumptions are made by the bioassay program RBDCOMP:

- AMAD is assumed to be $1 \mu \mathrm{m}$ if not supplied on the bioassay records.

Fố rưuitine computations:

- The measurement start and end dates in the form YYYYMMDD are used to bracket selection of measurements to be used for routine analysis.

- The intake window start and end dates in the form YYYYMMDD are used to bracket the computation of the intake.

- The analysis is carried out sequentially using the records encountered between the measurement start and end dates. Start and end exposure history records determine known periods of intake. Measurements in a no-intake period are used in the estimate of intake in previous intake periods. The intake rate is set to zero during a no-intake window. The intake rate for the period from a measurement to an end exposure history record is set to the intake rate determined in the previous intake period. Likewise, the intake rate from the last measurement to the global end date is set to the intake rate in the previous intake period.

- The protocol for analyzing routine records during a period of known intake assumes that the person has been exposed in a constant manner from the past measurement (or the global start date if the first measurement) up to the present measurement determined by the present measurement.

Additionally, for both routine and incident computations:

- All results are used in computing the intake. If there are fewer than two results above the lower critical level LC (see ref. 9), which is defined as one-half of the minimum detectable activity (MDA), the result of intake and dose is indicated as "NOT CONFIRMED"; otherwise it is indicated as "CONFIRMED." The ratio of MDA to LC (parameter RMDALC) is set in the SITE.DFT file. 


\subsection{DETAIIS OF OPERATION OF THE REPORT PROGRAM}

The REPORT program computes committed effective dose for each individual with records in the output records (DBS) file. REPORT writes three output files: (1) a file (with extension CED) containing the effective dose for each individual; (2) an output listing (REP) file, which is a complete listing by DBS record; and (3) a summary output (SUM) file, which contains only the committed dose by social security number.

The REPORT program first prints a header giving the version and date of the RBD program, the date of the computation, and the names of the output records (DBS) file and the bioassay data base file from which the records were extracted.

If you have previously computed committed effective dose reports, the output listing (REP and SUM) files will already exist. In this case the REPORT program asks if you wish to replace these files. If you respond $\mathrm{No}$, the program asks for a new name for the output files.

You are then asked for the type of screen display. Batch (selection 0 ) is used for batch processing. Weights used to compute intake and committed effective and organ doses from multiple assays for a given nuclide cannot be modified in this screen mode. Modify Weights (selection 1, the default) gives you information on the weights used to compute the committed dose for a given nuclide. You are prompted to modify the weights if the WGTMOD switch in the SITE.DFT file is set to true (T). Full (selection 2) gives more information than selection 1, including weighted intake and committed effective and organ doses by nuclide. As with selection 1 , the program also prompts you to modify weights if the WGTMOD switch in the SITE.DFT file is set to true (T).

\subsection{DETAIIS OF RBD OUTPUT LIST AND PRINT UTILITIES}

RBD main menu LIST and PRINT features allow listing and printing of RBD output files. These include the RBDCOMP listing (LOG) files and the REPORT listing (REP and SUM) files. In addition, you can list or print the nuclide file listings (LST files) and the retention/excretion plot listings (PLT files). Upon selecting RBD main menu item LIST RBD Work Files or PRINT RBD Work Files, you must select a file type (RBD Run Files, LST, or PLT files). When the selection RBD Run Files is chosen, a menu listing RBD runs is displayed, from which you select the desired run. If there are more than nine $\mathrm{RBD}$ run files to choose from, you can proceed to the next screen by selecting the line Next Screen for more files, pressing $N$, or pressing $<\mathrm{PgUp}\rangle$ or $\langle\mathrm{PgDn}\rangle$. This will then show a menu listing the files to be browsed (LOG, REP, and SUM files). The file selected will be listed to the screen or printed and marked. You may then delete all files with that filename, regardless of extension, by selecting the Delete all files menu item. To quit the LIST or PRINT utility, you must return to the file type selection menu (by pressing <Esc> repeatedly) and select Quit to Menu. 


\subsection{DETAIIS OF OPERATION OF RBD UTILITIES}

The RBD utility programs MODSITE, SORTREC, FILMAN, LISTNUC, and PLOTNUC are accessed from the RBD main menu.

RBD main menu item Modify SITE.DFT - MODSITE is used to modify the SITE.DFT file, that controls operation of the RBD software. MODSITE creates a copy of the existing SITE file, SITE.BAK, in the DBF subdirectory before modifying SITE.DFT. MODSITE prints a menu (see below) of the items you can alter.

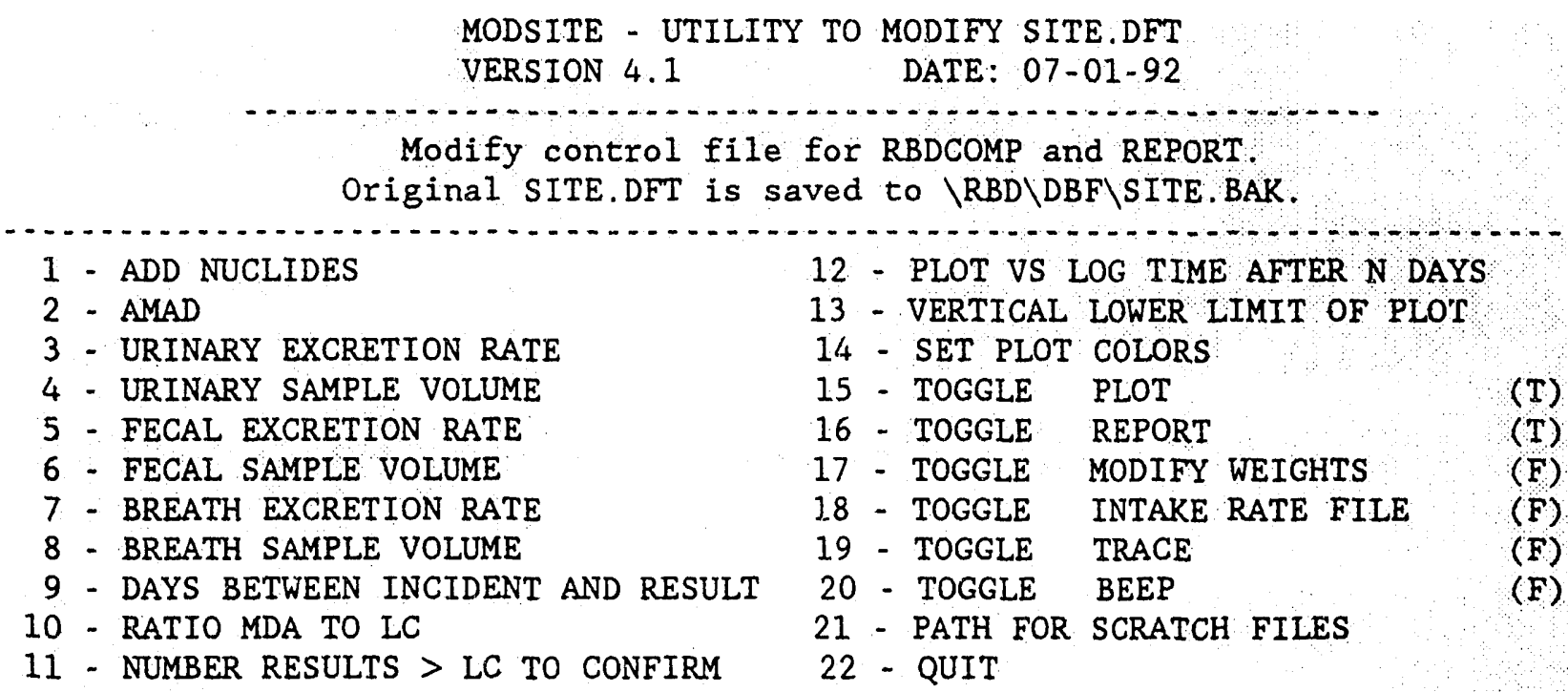

ENTER SELECTION: 15

PLOT UNIT RETENTION/EXCRETION FUNCTION TOGGLED TO FALSE

$* * x *$ Press <Enter> to continue $* * * *$

Items are selected from the menu by typing the item number and pressing <Enter $>$. Soine of these items are switches that can be toggled from true to false or false to true. The existing setcings are shown on the MODSITE menu. When a change is made to such a switch, the modification will show on the MODSITE menu when it is redisplayed. The other parameters in the SITE.DFT file are modified by selecting the menu item and typing in the modification. WARNING: MODSITE reads these modifications as character strings without checking the string. Be careful that numbers are typed correctly. If no modification is made, the original information is retained. When finished modifying SITE.DFT, select item 22 (Quit) to make the modifications permanent. 
You will get a chance to examine the modified SITE.DFT file. To make any corrections to SITE.DFT at this point, you must re-select MODSITE. If the program is aborted for any reason, a temporary file SITE.TMP in the DBF subdirectory will exist, and it must be deleted before running MODSITE again. The SITE.DFT file can also be modified using an editor.

Using MODSITE, you can alter default values assumed for parameters (urinary, fecal, and breath excretion rates and sample volumes, AMAD, etc.) missing from the bioassay record. You can also control program actions by toggling various switches. For example, if the PLOT switch (item 15) is toggled to true ( $T$ ), you will be able to plot the measurements per unit intake and the expected response per unit intake; if toggled to false $(F)$, no plot is produced. Likewise, if the REPORT switch (item 16) is toggled to true (T), the REPORT program will be run immediately after RBDCOMP; if toggled to false (F), the REPORT program is not run. Other switches turn on or off the ability to modify the assay weighting factors, the creation of intake rate files, a trace of the program actions, and whether the BEEP is heard when user response is required.

If additional radionuclides are added to the RBD software, you will need to modify the SITE.DFT file using MODSITE. It is recommended that you not make any alterations to the nuclide list in the SITE.DFT file unless directed to do so by the code developers. The SITE.DFT file allows modification of the ratio of minimum detectable activity (MDA) to the lower critical limit (LC). By default, this is set to 2. Modification may also be made to the number of results needed to confirm a computation of intake and dose. By default, there must be at least two results above the lower critical limit to confirm that an intake has occurred. Again you are cautioned against making a modification to these parameters unless instructed by the code developers.

The SITE.DFT file also contains parameters to control the plot of measurements per unit intake and the expected response per unit intake. These parameters are the duration (in days) when the time axis switches from linear to logarithmic, the lower limit of the y-axis, and the colors used in the plot. The following colors can be set:

$\begin{array}{lll} & \text { Default Color } & \text { Index } \\ \text { Foreground color } & \text { Light cyan } & 11 \\ \text { In vivo measurements and excretion function } & \text { Bright white } & 15 \\ \text { In vitro measurements and retention function } & \text { Yellow } & 14 \\ \text { Intake measurement window indicator } & \text { Light red } & 12 \\ \text { Start and end codes } & \text { Light magenta } & 13 \\ \text { Indication when measurements are below vertical limit } & \text { Light magenta } & 13\end{array}$

Lastly, using MODSITE you can alter the path for scratch files created during execution of RBDCOMP. These files are deleted on program completion and thus are different from RBD work files. 'The path for the scratch files is initially set to subdirectory WORK; however, you may wish to have scratch files written to a virtual drive. 
RBD main menu item Sort Records - SORTREC is used to append and sort bioassay records files into the order required by RBD. Records exported from the bioassay data base are automatically sorted in the proper order, but if two or more bioassay records files are appended and/or edited, SORTREC must be used to sort these records into the proper order required by RBD. SORTREC can also be used to append and sort RBD output (DBS) files and determine statistics on the number of records, number of individuals, and nuclides occurring in the combined and sorted file. This utility will also indicate when the assay weighting factors in the DBS file have been altered. The information is written to the file \$STAT.REC in subdirectory WORK.

First a list of bioassay records (DAT) files and output records (DBS) files is presented from which you select any number to be concatenated and sorted. You then select the type of file (DAT or DBS) to be sorted. You should note, however, that SORTREC is limited to a maximum of 7000 records. SORTREC asks for a filename for the resulting sorted file. SORTREC sorts records by SSN, department code, nuclide, class, reason code, incident date and time, and assay.

RBD main menu item File Manager - FILMAN is used to download and upload data base files and RBD work files. The download operation moves files from hard disk to floppy disk. The upload operation moves files from floppy disk to hard disk. When a data base is loaded, its associated files are automatically copied. All RBD work files are copied for the selected run. The work files may be deleted from the hard disk after being downloaded.

RBD main menu item Nuclide Data LIST Utility (LISTNUC utility) is used to list the contents of a nuclide data file to the screen and/or to a file (with extension LST). First a list of nuclides is presented from which you select the desired nuclide. You will then see the following screen:

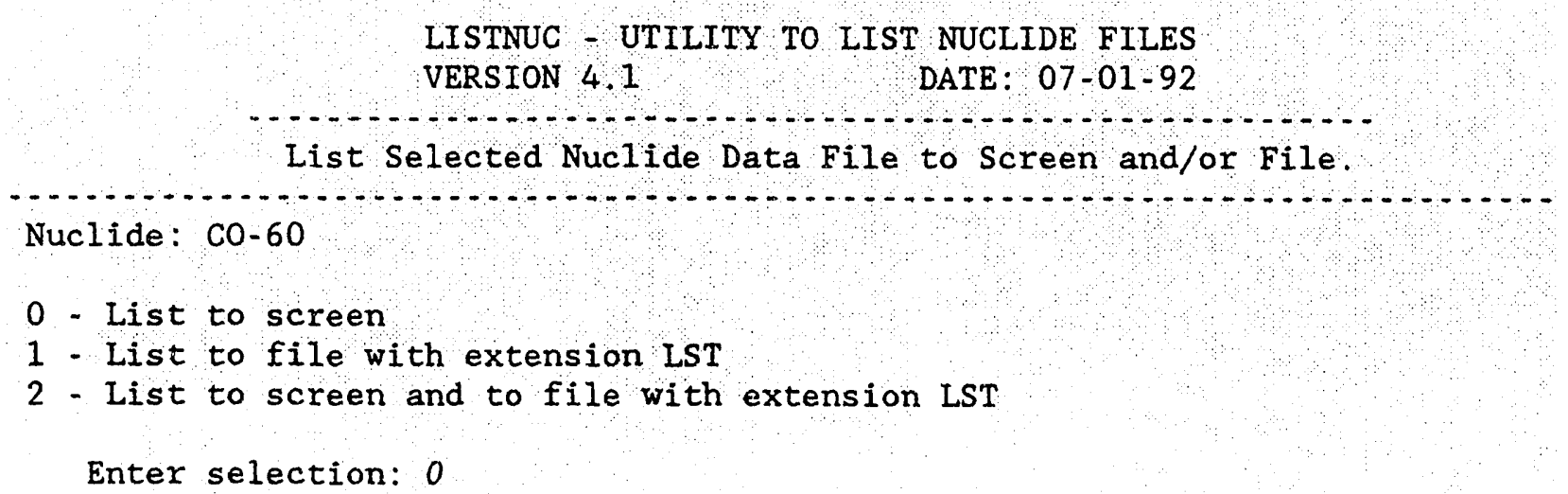

You can either list the nuclide file to the screen (0), to a file (1), or to both the screen and the file (2). If you select to list to a file (with extension LST) and the file already exists, you are asked if it should be replaced. The contents of the nuclide file listing (LST file) can be browsed or printed using RBD LIST or PRINT menu items. 
RBD main menu selection Unit Response PLOT utility (PLOTNUC utility) is used to plot the unit retention or excretion function for selected nuclide, class, pathway, and assay for incident or routine exposure. For example, to plot the unit excretion function in urine for inhalation of class $\mathrm{W}$ Co-60, select option 0 and enter the information shown below:

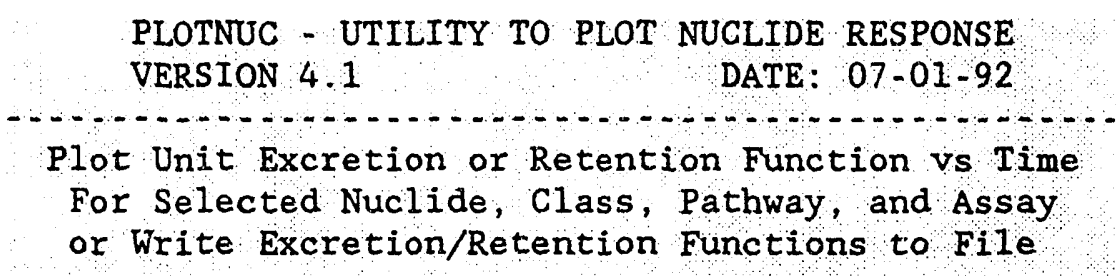

Nuclide. $60-60$

0 - Plot a retention/excretion curve

1 - Write retention and excretion plot data to file with extension PLT

Enter selection: 0

Enter Path (Inhalation-H, Ingestion-G, Wound-W): $H$

Classes available: $W \mathrm{Y}$

Enter Class: $W$

Enter AMAD (0.2 - 10.0 microns): 1

Enter REASON (Incident-1, Chronic-3): 1

Enter ASSAY (BRE, U, F, WBC, LUN, THY): $U$

Enter Maximum Time (in days) for plot (limit: 10000): 1000

The unit excretion plot for this case is shown in Fig. 11 on the following page.

The SITE.DFT file contains parameters that control features of the plots generated by the RBD software. The vertical axis on the plot is always a log axis. The horizonal axis can be either linear or log. Select menu item 12 on the MODSITE to control the time in days when the plot switches from linear to log. In the installed version of the SITE.DF $\Gamma$ file, this switch is set to 366 days. The lower limit on the vertical axis can be set by selecting item 13 on the MODSITE menu. This is set to $1 . E-7$ in the installed SITE.DFT. The colors of the plot can be set by selecting item 14 on the MODSITE menu (see above). 


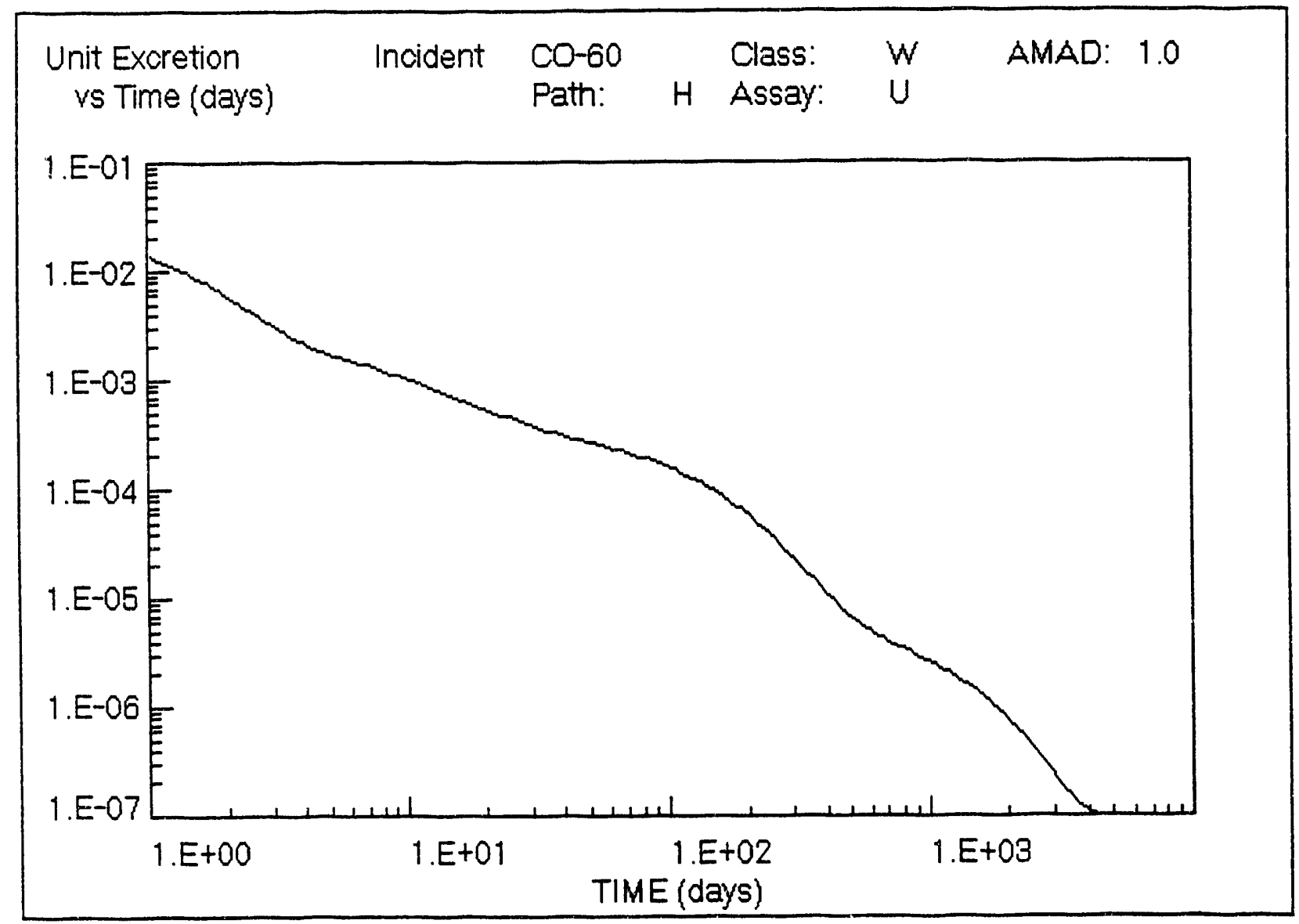

Fig. 11. Urinary excretion for chronic exposure to ${ }^{60} \mathrm{Co}$. 
RBD main menu selection Data Base Utility - EDITDBF is used to browse, edit or zap (remove the entries of) an RBD input or output data base (DBF) files. After selecting an RBD input data base (which automatically selects the corresponding output data base), you are presented with the following screen:

\begin{tabular}{|l|}
\hline \multicolumn{1}{|c|}{ EDITDBF Utility } \\
\hline Browse/Edit RBDTEST data base. \\
Zap the RBDTEST data base. \\
Browse/Edit OUTTEST data base. \\
Zap the OUTTEST data base. \\
Select another data base. \\
Quit and return to RBD main menu.
\end{tabular}

RBDTEST contains 30 records. OUTTEST contains 4 records

Select Browse/Edit RBDTEST data base to browse or edit that data base. Select Zap OUTTEST data base to remove the entries in the output data base OUTTEST. Do not do this until you have completed the RBD tutorial.

Extreme caution must be used when editing records in a bioassay or output data base or when zapping bioassay or output data bases. The RBD utility FILMAN can be used to archive RBD input and output data base files prior to modification. 


\section{ALGORITHMS FOR ESTIMATING INTAKE OF RADIONUCLIDES}

\subsection{INTRODUCTION}

We have developed a set of algorithms for estimating a worker's intake of a radionuclide based on measurements of activity within excreta or within the body at times after intake. The algorithms set forth here are similar to the formulations used in ICRP Publication 54 (ref. 4). In selecting and implementing the algorithms, the needs of quality assurance and the desire to limit future costs associated with maintenance of the resulting software were considered. The algorithms and the software have been subjected to extensive testing and verification.

\subsection{GENERAL APPROACH}

The software, entitled RBD, was designed to serve as a tool in demonstrating compliance with the occupational radiation protection guidance 10 CFR Part 20 (ref. 1). Even though some aspects of internal dosimetry will be further clarified in additional NRC guidance, the major thrust of the guidance deals with internal emitters, particularly the manner in which intakes and resultant doses are assessed and reported. Thus, we have focused our efforts toward estimating intakes, given measurements of the radionuclide in excreta and in the body.

We have assumed that the biokinetics governing the behavior of radionuclides within the body are linear such that the response of the body to an intake is directly proportional to the magnitude of the intake, and the response to continuous intakes can be viewed as a series of acute intakes. We describe the biokinetics by mathematical functions, and thus the software is not limited to any particular model or model structure. Therefore, the computational routines are independent of the biokinetic and mathematical details upon which the functions are based. The functions associated with each radionuclide are contained in data files, referred to as the nuclide files. To introduce new biokinetic information or to include a new bioassay procedure for a radionuclide, one need alter only the nuclide file; rarely should it be necessary to modify the software itself.

As an example, let $e_{u}(t)$ denote the urinary excretion rate at time $t$ following an instantaneous input of a unit activity into blood at $t=0$. If a unit intake into the body at $t=0$ results in the activity entering blood at a rate $g(t)$, then the expected urinary excretion rate, $E_{u}(t)$, at time $t$ is

$$
E_{u}(t)=\int_{0}^{t} g(\tau) e_{u}(t-\tau) d \tau
$$

The above integral represents the convolution of two functions; the convolution is defined as 
$f * g=\int_{0}^{t} g(\tau) f(t-\tau) d \tau$. The function $g(t)$ is often referred to as the forcing function (or stimulus) and $f(t)$ as the unit response function. The latter gives the response of the system to a unit input at time zero, while the convolution integral represents the response of the system to the forcing function.

For continuous intake at a rate $\dot{I}(t)$ during a period of length $T_{e}$, the expected urinary excretion rate $E_{u}^{c}\left(t, T_{e}\right)$ at time $t$ is given by

$$
E_{u}^{c}\left(t, T_{e}\right)=\int_{0}^{T_{e}} \dot{I}(\tau) E_{u}(t-\tau) d \tau
$$

If the intake was at a constant rate, $\dot{I}(t)=\dot{I}$, then

$$
\begin{aligned}
E_{u}^{c}\left(t, T_{e}\right) & =\dot{I} \int_{0}^{T_{e}} E_{u}(t-\tau) d \tau \\
& =\dot{I} \int_{t-T_{e}}^{t} E_{u}(\tau) d \tau .
\end{aligned}
$$

The above equation gives the excretion rate at any time $t$, including times beyond the intake period, that is, for $t>T_{e}$. Note that the lower limit of integration in Eq. (2b) is taken to be zero if $t<T_{e}$. 
Some properties of the convolution integral used in developing the algorithms are tabulated below.

\section{Algebra of Convolution}

\begin{tabular}{ll}
\hline Property & \multicolumn{1}{c}{ Definition } \\
\hline Commutative & $(f * g=g * f$ \\
Associative & $(k f) * g=k(f * g) ; k$ is a constant \\
Multiplicative & $\left(f_{1}+f_{2}\right) *\left(g_{1}+g_{2}\right)=$ \\
Distributive & $f_{1} * g_{1}+f_{1} * g_{2}+f_{2} * g_{1}+f_{2} * g_{2}$ \\
\hline
\end{tabular}

The functions describing the translocation of material within the body and the excretion rates can be derived from mathematical models of the biokinetics (e.g., the ICRP Task Group Lung Model ${ }^{5}$ ) or obtained frorn mathematical fits to observations (e.g., radon breath analysis for determination of radium). We have restricted the functions used in RBDCOMP to be of the form

$$
f(t)=\sum_{i=1}^{n} A_{i} e^{-a_{i} t}
$$

The functions, as defined in the nuclide data files, do not include radioactive decay. 
In relating the rate of excretion (or activity retained in the body) to the amount of material initially inhaled or ingested, we encounter convolutions of the form $f * g$ and $f * g * h$. We set out below the resulting expressions for the convolution integrals assuming $f(t)=\sum_{i} A_{i} e^{-a_{1} t}$, $g(t)=\sum_{j} B_{j} e^{-b_{j} t}$, and $h(t)=\sum_{k} C_{k} e^{-c_{k} t}$. The convolution $f * g$ yields

$$
f * g=\sum_{i, j} \Delta_{i, j}\left[e^{-b_{j} t}-e^{-a_{i} t}\right]
$$

where $\Delta_{i, j}=\frac{A_{i} B_{j}}{a_{i}-b_{j}}$, and the $\sum_{i, j}$ denotes $\sum_{i} \sum_{j}$. The convolution $f * g * h$ yields

$$
f * g * h=\sum_{i, j, k} \Delta_{i, j} C_{k}\left[\frac{e^{-c_{k} t}-e^{-b_{j} t}}{b_{j}-c_{k}}-\frac{e^{-c_{k} t}-e^{-a_{i} t}}{a_{i}-c_{k}}\right],
$$

where the summation is a triple sum over the indices $i, j$, and $k$.

If radioactive decay, as characterized by the decay constant $\lambda$, is applicable to both the functions in the convolution then

$$
f(t) e^{-\lambda t} * g(t) e^{-\lambda t}=(f * g) e^{-\lambda t}
$$

$$
\text { and } f(t) e^{-\lambda t} * g(t) e^{-\lambda t} * h(t) e^{-\lambda t}=(f * g * h) e^{-\lambda t}
$$

Consideration of chronic intakes involves a time integral of the response during the intake period [see Eqs. (2a) and (2b)], including radioactive decay as given by $e^{-\lambda t}$. Thus one needs to evaluate the integral of Eq. (4a): 


$$
\begin{gathered}
\int_{t-T_{e}}^{t}(f * g) e^{-\lambda \tau} d \tau \\
=\sum_{i, j} \Delta_{i, j}\left[\frac{e^{-\left(b_{j}+\lambda\right)\left(t-T_{e}\right)}-e^{-\left(b_{j}+\lambda\right) t}}{b_{j}+\lambda}-\frac{e^{-\left(a_{i}+\lambda\right)\left(t-T_{e}\right)}-e^{-\left(a_{i}+\lambda\right) t}}{a_{i}+\lambda}\right],
\end{gathered}
$$

where $\Delta_{i, j}$ and $\sum_{i, j}$ are defined above. In some instances one needs to integrate Eq. (4b):

$$
\begin{gathered}
\int_{t-T_{e}}^{t}(f * g * h) e^{-\lambda t} d \tau \\
=\sum_{i, j, k} \Delta_{i, j} C_{k}\left[\frac{e^{-\left(c_{k}+\lambda\right)\left(t-T_{e}\right)}-e^{-\left(c_{k}+\lambda\right) t}}{\left(b_{j}-c_{k}\right)\left(c_{k}+\lambda\right)}-\frac{e^{-\left(b_{j}+\lambda\right)\left(t-T_{c}\right)}-e^{-\left(b_{j}+\lambda\right) t}}{\left(b_{j}-c_{k}\right)\left(b_{j}+\lambda\right)}\right] \\
-\sum_{i, j, k} \Delta_{i, j} C_{k}\left[\frac{e^{-\left(c_{k}+\lambda\right)\left(t-T_{a}\right)}-e^{-\left(c_{k}+\lambda\right) t}}{\left(a_{i}-c_{k}\right)\left(c_{k}+\lambda\right)}-\frac{e^{-\left(a_{i}+\lambda\right)\left(t-T_{e}\right)}-e^{-\left(a_{l}+\lambda\right) t}}{\left(a_{i}-c_{k}\right)\left(a_{i}+\lambda\right)}\right],
\end{gathered}
$$

where $\Delta_{i, j}$ and $\sum_{i, j, k}$ are defined above. Equations (4) and (5) are the basic algorithms used by RBDCOMP. As outlined below, these equations are repeatedly applied to the various transfer and excretion functions included in nuclide files. The functions contained in the nuclide files are listed in Table 2. In all cases the functions are relative to a unit activity at $t=0$. 
Table 2. Functions used in analysis of bioassay measurements

\begin{tabular}{ll}
\hline Notation & \multicolumn{1}{c}{ Definition } \\
\hline$f_{\text {lung } \rightarrow \text { blood }}$ & Transfer rate from lung to blood \\
$f_{\text {lung }-G I}$ & Transfer rate from lung to GI Tract \\
$f_{G I-\text { blood }}$ & Transfer rate from GI tract to blood \\
$f_{G I-\text { feces }}$ & Transfer rate from GI tract to feces \\
$f_{\text {wound }- \text { blood }}$ & Transfer rate from wound to blood \\
$e_{u}$ & Urinary excretion rate of systemic activity \\
$e_{f}$ & Fecal excretion rate of systemic activity \\
$e_{b}$ & Breath excretion rate of systemic activity \\
$R_{\text {lung }}$ & Retention of activity in lung \\
$R_{G I}$ & Retention of activity in GI tract \\
$R_{\text {systemic }}$ & Retention of systemic activity \\
$R_{\text {organ }}$ & Retention of activity in organ (e.g., thyroid) \\
$R_{\text {wound }}$ & Retention at wound site \\
\hline
\end{tabular}




\subsection{EXCRETION AND RETENTION EQUATIONS}

Radionuclides are assumed to enter the body via inhalation, ingestion, or through the skin via wounds. The latter intake is assumed to occur as an isolated incident (acute exposure); inhalation and ingestion intakes may be either acute or chronic (continuous) in time. This section details the algorithms for the expected values for each assay considered within $\mathrm{RBD}$, namely, urinary excretion, fecal excretion, breath excretion, and in vivo measurements.

\subsubsection{Urinary Excretion}

\section{Inhalation:}

Let $e_{u}(t)$ represent the urinary excretion rate at time $t$ following introduction of a unit amount of the radionuclide into blood at $t=0$ (i.e., unit response function without radioactive decay). In the case of inhalation, the inputs to blood are due to material cleared directly from the lung, at rates denoted by $f_{\text {lung-blood }}(t)$, and material entering from the GI tract at rates denoted by $f_{G l \rightarrow b l o o d}(t)$. The material in the GI tract is cleared from the lung at rates denoted by $f_{\text {lung } \rightarrow G I}(t)$. The expected excretion rate, $E_{\alpha}^{a}(t)$, at time $\mathrm{t}$ following an acute intake of a unit activity by inhalation at $t=0$ of a radionuclide with decay constant $\lambda$ is

$$
E_{u}^{a}(t)=e^{-\lambda t}\left[f_{\text {lung }- \text { blood }}(t)+f_{\text {lung }-G I}(t) * f_{G I-\text { blood }}(t)\right] * e_{u}(t)
$$

Equation (6) is evaluated using Eqs. (4a) and (4b). For brevity we have dropped the time argument of the transfer rates in the equations below.

If an individual chronically inhales activity at a uniform rate during a period of length $T_{e}$, then the excretion rate at time $t$ per unit intake rate, $E_{k}^{c}\left(t, T_{e}\right)$, is

$$
\begin{aligned}
E_{u}^{c}(t) & =\int_{t-T_{e}}^{t} E_{u}^{a}(x) d x \\
& =\int_{t-T_{e}}^{t} e^{-\lambda x}\left[f_{\text {lung }- \text { blood }}+f_{\text {lung }-G I} * f_{G I-\text { blood }}\right] * e_{u} d x .
\end{aligned}
$$




\section{Ingestion:}

For an acute intake by ingestion, the fractional rate at which activity enters blood from the gastrointestinal tract is denoted by $f_{G l-b l o o d}(t)$. The expected excretion rate, $E_{u}^{a}(t)$, at time $t$ per unit intake at $t=0$ for a radionuclide with decay constant $\lambda$ is

$$
E_{u}^{a}(t)=e^{-\lambda t}\left[f_{G I-\text { blood }} * e_{u}\right]
$$

Equation (8) is evaluated using Eq. (4a).

If an individual chronically ingests activity at a uniform rate during a period of length $T_{e}$, then the urinary excretion rate at time $t$ per unit intake rate, $E_{u}^{c}\left(t, T_{e}\right)$, is

$$
\begin{aligned}
E_{u}^{c}\left(t, T_{e}\right) & =\int_{t-T_{e}}^{t} E_{u}^{a}(x) d x \\
& =\int_{t-T_{e}}^{t} e^{-\lambda x}\left[f_{G I-\text { blood }} * e_{k}\right] d x .
\end{aligned}
$$

Equation (9) is evaluated using Eq. (5a).

\section{Wound intake:}

Let $f_{\text {wound-blood }}(t)$ denote the rate at which activity is absorbed into blood from the wound site. The rate of urinary excretion $E_{u}(t)$ per unit activity at the wound site is initially

$$
E_{u}(t)=e^{-\lambda z}\left[f_{\text {wound }-b l o o d} * e_{u}\right]
$$

Equation (10) is evaluated using Eq. (4a).

\subsubsection{Fecal Excretion}

\section{Inhalation:}

Let $f_{\text {lung }- \text { blood }}(t)$ denote the fractional rate at which activity inhaled at $t=0$ is transferred to blood and $e_{f}$ denote the fecal excretion rate following introduction of a unit activity into blood at $t=0$. In addition to the fecal excretion of activity that has entered the systemic pool, there will also be a component associated with material that has passed unabsorbed through the GI tract, which is denoted as $f_{G l-f e c e s}(t)$. The expected fecal excretion rate, $E_{f}^{a}(t)$, at time $t$ following an acute intake 
of a unit activity by inhalation of a radionuclide with decay constant $\lambda$ is

$$
E_{f}^{a}(t)=e^{-\lambda t}\left[\left(f_{\text {lung }- \text { blood }}+f_{\text {lung }-G I} * f_{G I-b l o o d}\right) * e_{f}+f_{\text {lung }-G I} * f_{G I-\text { feces }}\right]
$$

Equation (11) is evaluated using Eqs. (4a) and (4b).

If an individual chronically inhales activity at a uniform rate during a period of length $T_{e}$, then the excretion rate at time $t$ per unit intake rate, $E_{f}^{c}\left(t, T_{e}\right)$, is given as

$$
\begin{aligned}
E_{f}^{c}\left(t, T_{e}\right) & =\int_{t-T_{e}}^{t} E_{f}^{a}(x) d x \\
& =\int_{t-T_{e}}^{t} e^{-\lambda x}\left[\left(f_{\text {lung }- \text { blood }}+f_{\text {lung }-G I} * f_{G I-\text { blood }}\right) * e_{f}+f_{\text {lung }-G I} * f_{G I-\text { feces }}\right] d x .
\end{aligned}
$$

Equation (12) is evaluated using 5qs. (5a) and (5b).

\section{Ingestion:}

Let $f_{G I-b l o o d}(t)$ denote the fractional rate at which activity ingested at time $t=0$ is transferred to blood. The expected fecal excretion rate, $E_{f}^{a}(t)$, following an acute intake of a unit activity by ingestion at $t=0$ is

$$
E_{f}^{a}(t)=e^{-\lambda t}\left[f_{G I-b l o o d} * e_{f}+f_{G I-\text { feces }}\right]
$$

Equation (13) is evaluated using Eq. (4a).

If an individual chronically ingests activity at a uniform rate during a period of length $T_{e}$, then the excretion rate at time $t$ per unit intake rate, $E_{f}^{c}\left(t, T_{e}\right)$, is 


$$
\begin{aligned}
E_{f}^{c}\left(t, T_{e}\right) & =\int_{t-T_{e}}^{t} E_{u}^{a}(x) d x \\
& =\int_{t-T_{e}}^{t} e^{-\lambda x}\left[f_{G I-\text { blond }} * e_{f}+f_{G I-\text { feces }}\right] d x .
\end{aligned}
$$

Equation (14) is evaluated using Eq. (5a).

\section{Wound Intake:}

Let $f_{\text {mound }-b l o o d}(t)$ denote the fractional rate at which activity is absorbed into blood from the wound site. The rate of fecal excretion, $E_{f}(t)$, per unit activity initially at the wound site is given as

$$
E_{f}(t)=e^{-\lambda t}\left[f_{\text {wound }- \text { blood }} * e_{f}\right]
$$

Equation (15) is evaluated using Eq. (4a).

\subsubsection{Breath Excretion}

\section{Inhalation Intake:}

Let $f_{\text {lung-blood }}(t)$ denote the fractional rate at which activity inhaled at $t=0$ is transferred to blood and $e_{b}(t)$ the rate of excretion of the radionuclide in breath following introduction of a unit activity into blood at $t=0$. The expected breath excretion rate, $E_{b}^{a}(t)$, at time $t$ following an acute intake of a unit activity by inhalation of a radionuclide with decay constant $\lambda$ is

$$
E_{b}^{a}(t)=e^{-\lambda t}\left[\left(f_{\text {lung }- \text { blood }}+f_{\text {lung }-G I} * f_{G I-\text { blood }}\right) * e_{b}\right]
$$

Equation (16) is evaluated using Eqs. (4a) and (4b).

If an individual chronically inhales activity at a uniform rate during a period of length $T_{e}$, then the excretion rate at time $t$ per unit intake rate, $E_{b}^{c}\left(t, T_{e}\right)$, is given as 


$$
\begin{aligned}
E_{b}^{c}\left(t, T_{e}\right) & =\int_{t-T_{c}}^{t} E_{b}^{a}(x) d x \\
& =\int_{t-T_{e}}^{t} e^{-\lambda x}\left[\left(f_{\text {lung }- \text { blood }}+f_{\text {lung }-G I} * f_{G I-\text { blood }}\right) * e_{b}\right] d x
\end{aligned}
$$

Equation (17) is evaluated using Eqs. (5a) and (5b).

\section{Ingestion Intake:}

Let $f_{G l-b l o o d}(t)$ denote the fractional rate at which a unit activity ingested at time $t=0$ is transferred to blood. The expected excretion rate in breath, $E_{b}^{a}(t)$, at time $t$ following an acute intake of a unit activity by ingestion at $t=0$ is

$$
E_{b}^{a}(t)=e^{-\lambda t}\left[f_{G I-b h r a d} * e_{b}\right]
$$

Equation (18) is evaluated using Eq. (4a).

If an individual chronically ingests activity at a uniform rate during a period of length $T_{e}$, then the excretion rate at time $t$ per unit intake rate, $E_{b}^{c}\left(t, T_{e}\right)$, is

$$
\begin{aligned}
E_{b}^{c}\left(t, T_{e}\right) & =\int_{t-T_{e}}^{t} E_{b}^{a}(x) d x \\
& =\int_{t-T_{c}}^{t} e^{-\lambda x}\left[f_{G I-b l o o d} * e_{b}\right] d x .
\end{aligned}
$$

Equation (19) is evaluated using Eq. (5a).

\section{Wound Intake:}

Let $f_{\text {wound-blood }}(t)$ denote the fractional rate at which activity is absorbed into blood from the wound site. The rate of excretion in breath, $E_{b}(t)$, per unit activity initially at the wound site is given as

$$
E_{b}(t)=e^{-\lambda t}\left[f_{\text {wound }-b l o o d} * e_{b}\right]
$$

Equation (20) is evaluated using Eq. (4a). 


\subsubsection{Organ Burdens}

\section{Lung:}

Let $\boldsymbol{R}_{\text {buge }}(t)$ denote the fraction of the activity inhaled at $t=0$ that is retained in the lung at later times without consideration of radioactive decay. If we assume that $R_{\text {bog }}(t)=\sum_{i=1}^{n} A_{i} e^{-a_{1} t}$, the activity present in the lung at time $t, q_{\text {bang }}^{a}(t)$, following an acute intake of unit activity at $t=0$ is

$$
q_{\text {luns }}^{e}(t)=e^{-\lambda s} R_{\text {leng }}(t)=\sum_{i=1}^{n} A_{i} e^{-\left(e_{i}+\lambda\right) t} .
$$

Equation (21) is also applicable to retention at the wound site with $R_{\text {mownd }}(t)$ replacing $R_{\text {lugs }}(t)$.

If the intake occurs at a constant rate during a period of length $T_{e}$, then the lung burden $q_{\text {barg }}^{c}\left(t, T_{e}\right)$ per unit intake rate is

$$
\begin{aligned}
q_{\text {lung }}^{c}\left(t, T_{e}\right) & =\int_{t-T_{i}}^{t} q_{\text {lung }}^{a}(x) d x \\
& =\sum_{i} \frac{A_{i}}{a_{i}+\lambda}\left[e^{-\left(a_{i}+\lambda\right)\left(t-T_{e}\right)}-e^{-\left(a_{i}+\lambda\right) t}\right] .
\end{aligned}
$$

Systemic organs (e.g., thyroid):

Let $\boldsymbol{R}_{\text {orgen }}(t)$ denote the activity present in a systemic organ following introduction of a unit activity into blood at time $t=0$, without consideration of radioactive decay. The activity present in the organ, $q_{\mathrm{organ}}^{a}(t)$, following an inhalation intake of a unit activity at $t=0$ is

$$
q_{\text {organ }}^{a}(t)=e^{-\lambda t}\left[f_{\text {lung }- \text { blood }}+f_{\text {lung }-G I} * f_{G I-\text { blood }}\right] * R_{\text {organ }}(t)
$$


If the intake was by ingestinn, then the activity present in the organ is

$$
q_{\text {organ }}^{a}(t)=e^{-\lambda t}\left[f_{G I-b l o o d} * R_{\text {organ }}(t)\right]
$$

If the intake occurred at a constant rate during a period of length $T_{e}$, then the organ burden $q_{\text {organ }}^{c}\left(t, T_{e}\right)$ at any time $t$ per unit intake rate by inhalation is

$$
\begin{aligned}
q_{\text {organ }}^{c}\left(t, T_{e}\right) & =\int_{t}^{t}-T_{e}^{a_{\text {argan }}^{a}}(x) d x \\
& =\int_{t}^{t}-T_{e}^{e^{-\lambda x}}\left[f_{\text {lung - blood }}+f_{\text {lung }-G I} * f_{G I-\text { blood }}\right] * R_{\text {organ }} d x,
\end{aligned}
$$

and by ingestion,

$$
q_{\text {organ }}^{c}\left(t, T_{e}\right)=\int_{t-T_{e}}^{t} e^{-\lambda x}\left[f_{G I-\text { blood }} * R_{\text {organ }}\right] d x
$$

These equations can be evaluated using Eqs. (5a) and (5b). 


\subsection{APPLICATION OF THE BIOASSAY ALGORITHMS}

The above algorithms form the bases of the RBDCOMP program. The majority of the numerical calculations center around evaluation of Eqs. (4) and (5) as required by the pathways of the nuclide within the body. The RBD software considers two types of exposure. Acute or incident exposures are considered the well defined in terms of the time of the exposure, and specific bioassay measurements ary underiaken for the purpose of estimating the magnitude of the intake. Chronic or routine exposures arise from expected intake due to low-level contamination in the work environment (e.g., chronic airborne activity). Following is a brief outline of the final steps in the calculations of intake.

Acute intake:

The estimated activity $\langle I\rangle$ of a radionuclide taken into the body in an incident (acute intake) is derived as

$$
<I>=\frac{1}{N} \sum_{i=1}^{N} \frac{Y(t)}{E^{a}(t)},
$$

where $Y(t)$ denotes the measured excretion rate (or body burden) at time $t, E^{a}(t)$ is the expected excretion rate (or body burden) at time $t$ for a unit intake, and $N$ is the total number of measurements. Note that no weighting of the measurements is employed in estimating the intake.

Chronic intake:

Consider $n$ bioassay measurements $\left\{Y_{1}, Y_{2}, \ldots, Y_{n}\right\}$ obtained at times $\left\{T_{1}, T_{2}, \ldots, T_{n}\right\}$ during a period of chronic exposure. Assume that the exposure ended at $T_{f}\left(T_{f}>T_{n}\right)$ and that $m$ additional

measurements $\left\{Y_{n+1}, Y_{n+2}, \ldots Y_{n+m}\right\}$ were obtained at times $\left\{T_{n+1}, T_{n+2}, \ldots T_{n+m}\right\}$ post-exposure. The intake rate during the period $T_{i-1}$ to $T_{i}$ for $T_{i}<T_{f}$ is computed as

$$
\dot{I}_{i}=\frac{Y_{i}(t)-\sum_{j=1}^{i-1} \dot{I}_{j} E^{c}\left(\Delta T_{j}, T_{i}-T_{j-1}\right)}{E^{c}\left(\Delta T_{i}, \Delta T_{i}\right)},
$$


where $\Delta T_{i}=T_{i}-T_{i-1}$ and $E^{c}(x, y)$ is the expected excretion rate (or body burden) at time $y$ for uniform intake at a unit rate during a period of duration $x$. We assign the intake rate $\dot{I}_{n}$ estimated for the period $\left(T_{n-1}\right.$ to $\left.T_{n}\right)$ to the period $\left(T_{n}\right.$ to $\left.T_{f}\right)$, that is, from the last measurement to the end of exposure. The estimated intake, $\left\langle I_{T}\right\rangle$, derived from the measurements during the exposure period is then

$$
<I_{T}>=\sum_{i}^{n} \dot{I}_{i}\left(T_{i}-T_{i-1}\right)+\dot{I}_{n}\left(T_{f}-T_{n}\right)
$$

Each post-exposure measurement provides additional information regarding the total intake. Using the intake rate vector $\left(\dot{I}_{i}, i=1, n\right)$ estimated from Eq. (28), the predicted excretion at time $T_{i+n}$ beyond the exposure period as

$$
<Y_{i+n}>=\sum_{j=1}^{n} \dot{I}_{j} E^{c}\left(\Delta T_{j}, T_{i+n}-T_{j-1}\right)
$$

We obtain a new estimate of the total intake from the post-exposure measurement $Y_{i+n}$ as

$$
<I_{T}>_{i}=\frac{Y_{i+n}}{\sum_{j=1}^{n} i_{j} E^{c}\left(\Delta T_{j}, T_{n+1}-T_{j-1}\right)},
$$

where $i_{j}$ is the fraction of the current estimated total intake in the period $\Delta T_{j}$ and is given by $\left.\dot{I}_{j} /<I_{T}\right\rangle$, where $\left\langle I_{T}\right\rangle$ is the current estimate of the total intake and $\dot{I}_{j}$ is the intake rate during the $j$ th period. A new estimate of the total intake is obtained as the weighted average of the current estimate and the value derived from the $i$ th post-exposure measurement as

$$
\left.\left\langle I_{T}\right\rangle=\frac{1}{n+i}\left[(n+i-1)<I_{T}\right\rangle+\left\langle I_{T}\right\rangle_{i}\right] .
$$

This procedure is repeated over all post-exposure measurements. Note that the intake pattern derived from measurements during the exposure period is preserved; post-exposure measurements only improve the estimate of the total intake and do not change the fraction of intake during each time step. 


\section{TECHNICAL DESCRIPTION OF NUCLIDE DATA FILES}

\subsection{INTRODUCTION}

The general principles of monitoring for the radiation protection of workers have been set forth in various reports of the ICRP ${ }^{2.5}$ and NCRP. ${ }^{6}$ Monitoring of individuals for internal exposure is based on the measurement of radionuclides in the whole body (or specific organs/regions) and on the measurement of radionuclides in excreta. For quantitative assessment of internal exposure, the results of these measurements must be translated into quantities that can be compared with the primary radiation protection guidance (limits on annual effective dose equivalent, committed effective dose equivalent, or committed dose equivalent for specific organs) or to the secondary quantities (Annual Limits on Intake - ALI). ${ }^{5}$ The relationships between levels in the body or in excreta and the estimated intake and resulting dose can only be established through use of biokinetic and dosimetric models describing the behavior of the material in the body.

\subsection{MODEL OF THE RESPIRATORY SYSTEM}

This report uses the compartmental model of the respiratory system presented in ICRP Publication 30 (ref. 5) to describe the retention in the lung of inhaled aerosols and the rate of translocation of deposited material to other regions of thie body.

The respiratory system is divided into three regions into which the aerosol may deposit - the nasal passages $(N P)$, the trachea and bronchial tree $(T B)$, and the pulmonary parenchyma $(P)$. An additional region $(L)$ represents the lymphat $c$ tissue of the respiratory system. The fraction of the inhaled activity deposited in each region i $s$ umed to vary with the aerodynamic properties of the aersol. The fractions deposited in the $N P, 1:$, and $P$ regions are denoted by $D_{N P}, D_{T B}$, and $D_{P}$, respectively. The shortfall $\left(1-D_{N P}-D_{T B}-D_{P}\right)$ is exhaled and thus not retained in the respiratory tract. The deposition fractions, as a function of the activity median aerodynamic diameter (AMAD) of an aerosol with a log-normal distribution of diameters, are shown in Fig. 12.

Materials have been classified according to their rate of clearance from the pulmonary region of tic respiratory tract. Materials with a half-time of less than 10 days are classified as $D$ (day); those with half-times between 10 and 100 days are classified as $W$ (weeks); and those with greater half-times are classified as $Y$ (year). The clearance model of the respiratory tract is shown in Fig. 13. 


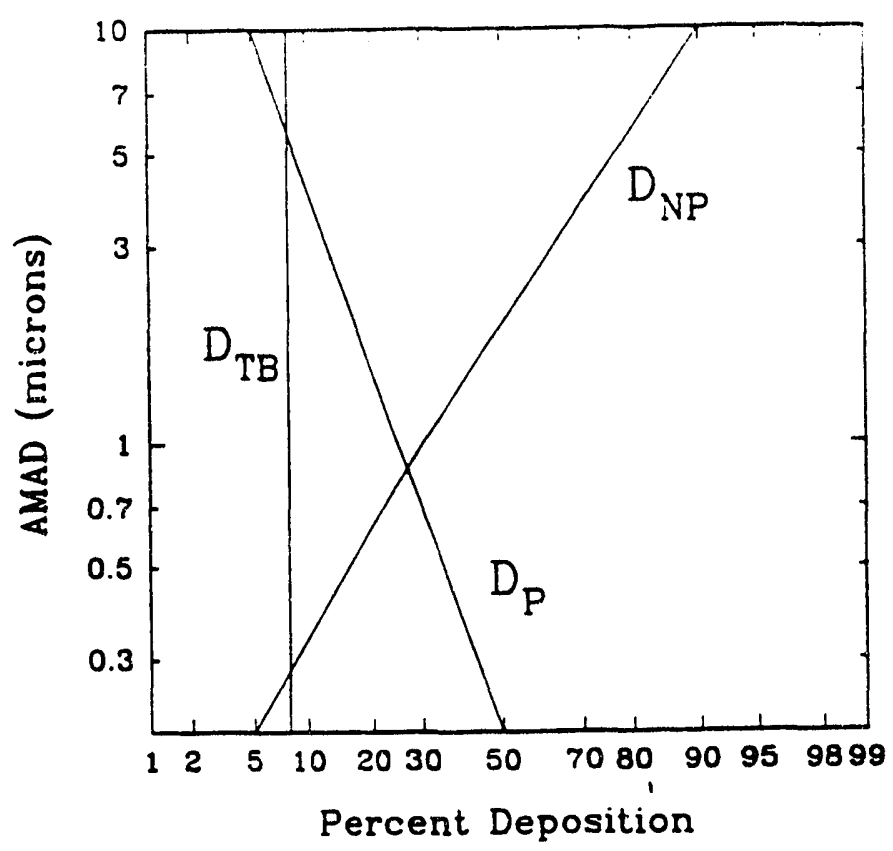

Fig. 12. Deposition of dust in the respiratory system. The percentage of activity or mass of an aerosol which is deposited in the nasal passages $\left(D_{\mathrm{NP}}\right)$, the trachea and bronchial tree $\left(\mathrm{D}_{\mathrm{TB}}\right)$, and the pulmonary parenchyma $\left(D_{P}\right)$ is given in relation to the activity median aerodynamic diameter (AMAD) of the aerosol distribution (in microns).

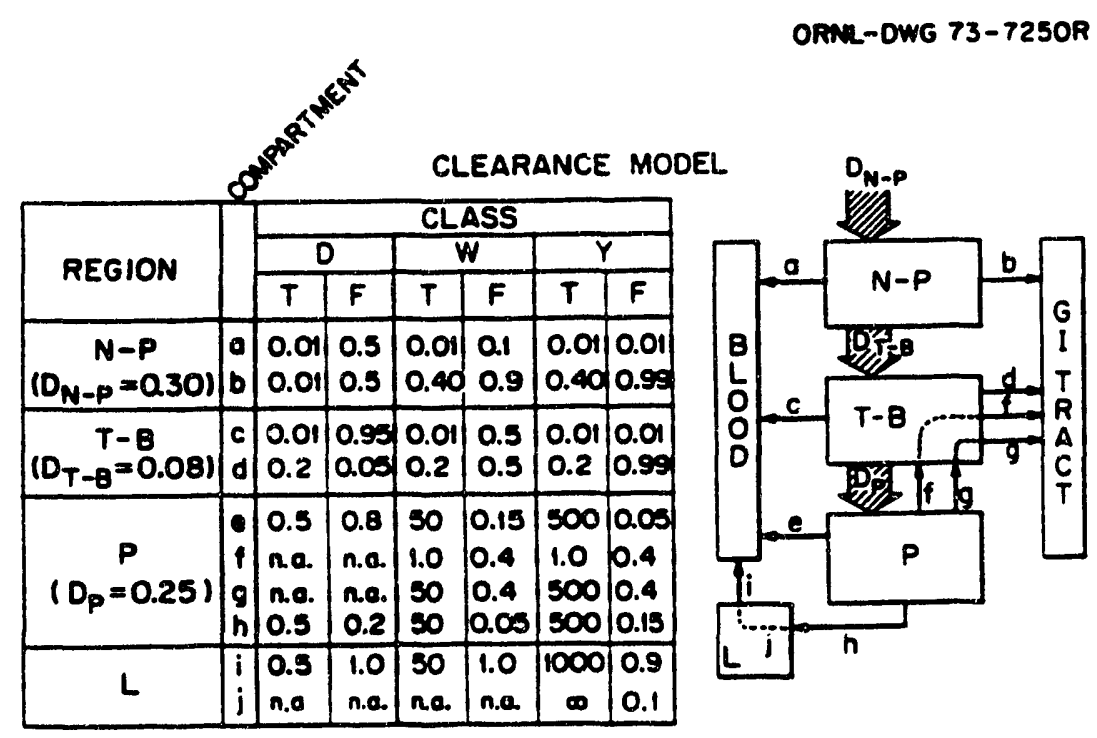

Fig. 13. The values for the removal half times, $T$, in days, and compartmental fractions, $F$, for the ICRP lung model for $\mathrm{D}, \mathrm{W}$, and $\mathrm{Y}$ class material. An AMAD of $1 \mu \mathrm{m}$ is assumed. The schematic diagram identifies the various clearance compartments (a-j) in the lung model. The entry n.a. indicates not applicable. 
Each compartment $(a-j)$ is associated with a particular pathway of clearance with a half-time for biological clearance of $T_{i}$ and associated pathway fraction $F_{i}$. The values $T_{i}$ and $F_{i}$, for the three clearance classes, are given in Fig. 13. Deposited material is taken up by the body from the respiratory tract through compartments $a, c, e$, and $i$. Compartments $b, d, f$, and $g$ are associated with mechanical transport of material into the gastrointestinal (GI) tract. The GI tract is discussed below.

The clearance of material from each compartment is assumed to be described by first-order kinetics at rates given by the rate constant $\lambda_{i}$, which is related to the half-time $T_{i}$ as

$$
\lambda_{i}=\frac{\ln (2)}{T_{i}} \text {, }
$$

where $\ln (2)$ is the natural logarithm of $2(\sim 0.693)$. The clearance of inhaled material from the lung is therefore described by a set of coupled first-order differential equations.

\subsection{MATHEMATICAL SOLUTIONS TO MODELS FOR RESPIRATORY TRACT}

Functions describing the retention of inhaled activity in the respiratory tract and rate of transfer of inhaled activity to blood and to the $g$ istrointestinal tract were derived as indicated in ICRP Publication 30 (see Appendix A of ref. 5). The fraction of the inhaled activity present in the compartments of the respiratory tract, see Fig. 13, at any time t, excluding consideration of radioactive decay, can be expressed as

$$
\begin{aligned}
& q_{v}(t)=F_{v} D_{N P} e^{-\lambda_{v} t}, v=a \text { and } b \\
& q_{c}(t)=F_{c} D_{T B} e^{-\lambda_{c} t} \\
& q_{d}(t)=F_{d} D_{T B} e^{-\lambda_{d} t}+\frac{F_{f} T_{d} D_{P}}{T_{d}-T_{f}}\left[e^{-\lambda_{d} t}-e^{-\lambda_{f} t}\right]+\frac{F_{g} T_{d} D_{P}}{T_{d}-T_{g}}\left[e^{-\lambda_{d} t}-e^{-\lambda_{g} t}\right] \\
& q_{v}(t)=F_{v} D_{P} e^{-\lambda t}, v=e, f, g, \text { and } h \\
& q_{i}(t)=\frac{F_{h} F_{i} T_{i} D_{P}}{T_{i}-T_{h}}\left[e^{-\lambda_{i} t}-e^{-\lambda_{h} t}\right] \\
& q_{j}(t)=F_{j} F_{h} D_{P}\left[e^{-\lambda_{j} t}-e^{-\lambda_{h} t}\right] .
\end{aligned}
$$


The retention in the respiratory tract, $R_{\text {lung }}(t)$, is

$$
R_{\text {lung }}(t)=\sum_{v=a}^{j} q_{v}(t)
$$

where $q_{v}(t)$ are given by Eq. (34). The rate of transfer of inhaled activity to blood is

$$
f_{\text {lung } \rightarrow \text { blood }}(t)=\lambda_{a} q_{a}(t)+\lambda_{c} q_{c}(t)+\lambda_{e} q_{e}(t)+\lambda_{i} q_{i}(t),
$$

where $q_{a}(t), q_{c}(t), q_{e}(t)$, and $q_{i}(t)$ are given by Eq. (34). Inhaled activity is transferred to the gastrointestinal tract (stomach) at a rate

$$
f_{\text {lung } \rightarrow G I}(t)=\lambda_{b} q_{b}(t)+\lambda_{d} q_{d}(t)
$$

where $q_{b}(t)$ and $q_{d}(t)$ are given by Eq. (34).

If the difference between the half times in the denominator of the expressions for $q_{i}(t)$ and $q_{d}(t)$ (f Eq. (34) is zero, then division by zero occurs. Inspection of half-time values in Fig. 13 indicates this occurs only with $q_{i}(t)$ when considering clearance classes $\mathrm{D}$ and $\mathrm{W}$. The solution under these conditions will not be a sum of exponentials. An alternative computational approach, adopted here, involves separating the half times by an arbitrary factor. We have taken $T_{i}$ to be $1.002 T_{h}$ for classes $\mathrm{D}$ and $\mathrm{W}$.

To consider the influence of aerosol size on the various respiratory functions it is useful to structure the functions as

$$
f(t)=\sum_{v=a}^{i}\left[D_{N P} A_{N P, v}+D_{T B} A_{T B, v}+D_{P} A_{P, v}\right] e^{-\lambda_{v} t},
$$

where $D_{N P}, D_{T B}$, and $D_{P}$ denote the deposition fractions for the aerosol in the NP, TB, and $\mathrm{P}$ regions of the lung. These deposition fractions are dependent on the activity median aerodynamic diameter (AMAD) of the inhaled aerosol. For example, consider the function $f_{\text {ung }-G I}(t)$ for a class $\mathrm{Y}$ aerosol. The nonzero coefficients of this function are 


$$
\begin{aligned}
& A_{N P, b}=\lambda_{b} F_{b} \\
& A_{T B, d}=\lambda_{d} F_{d} \\
& A_{P, d}=\frac{\lambda_{d} F_{f} T_{D}}{T_{d}-T_{f}}+\frac{\lambda_{d} F_{g} T_{d}}{T_{d}-T_{g}} \\
& A_{P, f}=-\frac{\lambda_{d} F_{f} T_{d}}{T_{d}-T_{f}} \\
& A_{P, g}=-\frac{\lambda_{d} F_{g} T_{d}}{T_{d}-T_{g}} .
\end{aligned}
$$

These coefficients of Eq. (38) permit evaluation of the rate of transfer of inhaled activity for any size aerosol to blood, given the values for $D_{N P}, D_{T B}$, and $D_{P}$ (see Fig. 12). All respiratory functions have been formulated in a similar manner.

The respiratory functions were checked with the explicit solutions of the differential equations governing the kinetics obtained using the DIFSOL program of Killough and Eckerman (ref. 12). The respiratory functions are contained in the nuclide data files.

\subsection{MODEL OF THE GASTROINTESTINAL TRACT}

Radionuclides reach the gastrointestinal tract either directly by ingestion or indirectly by translocation from the respiratory tract, as described above. This report uses the compartmental model of the gastrointestinal tract described in ICRP Publication 30 (ref. 5).

The gastrointestinal tract is represented by four sections. Each section is considered as a single compartment, and translocation of materials from one compartment to the next is assumed to be governed by first-order kinetics. Rate constants, $\lambda_{i}$, for transfer out of the compartments, are given in Fig. 14. We have assumed that the uptake of material from the GI tract to body fluids will occur from the small intestine (SI). The rate constant, $\lambda_{s}$, for the translocation of activity to the systemic pool (blood), can be estimated from the $f_{1}$ parameter, which is the fraction of the stable element reaching body fluids following ingestion:

$$
\lambda_{s}=\frac{f_{1} \lambda_{S I}}{1-f_{1}} .
$$

If an $f_{l}$ value of 1 is assigned, then the singularity in the above equation can be avoided by assuming that the material enters blood directly from the stomach. Here, however, we have arbitrarily limited $f_{1}$ to values less than or equal to 0.95 . The $f_{l}$ values are those assigned by the ICRP in Publication 30 . 
ORNL-DWG 77-2742R

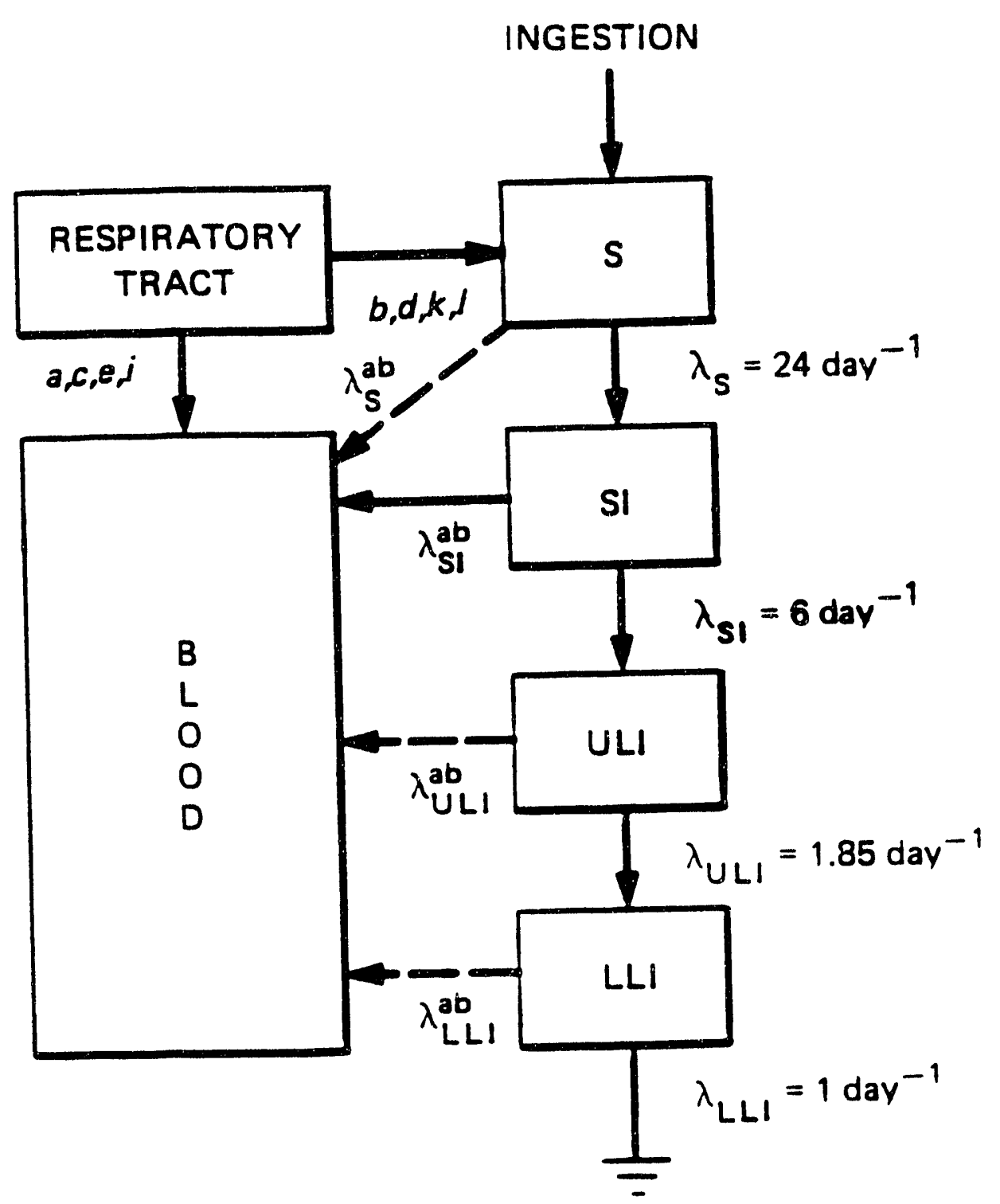

Fig. 14. Schematic representation of movement of radioactivity among respiratory tract, GI tract, and blood with transfer rates between compartments, $\lambda_{i}$, shown. 


\subsection{MATHEMATICAL SOLUTIONS TO MODELS FOR THE GASTROINTESTINAL TRACT}

Functions describing the retention of ingested activity and the rates of transfer of ingested activity to blood and to feces were derived assuming a unit activity present in the stomach at $t=0$. These functions are calculated for the value of $f_{1}$ considered to be applicable to the particular radionuclide. The gastrointestinal model is shown in Fig. 14. The fraction of the ingested activity present at any time $t$ in the segments of the GI tract, excluding radioactive decay, can be written as

Stomach (ST):

$$
q_{S T}(t)=e^{-\lambda_{S T} t}
$$

Small intestine (SI):

$$
q_{S I}(t)=\lambda_{S T}\left[\frac{e^{-\lambda_{S I} t}-e^{-\lambda_{S I} t}}{\lambda_{S I}^{\prime}-\lambda_{S T}}\right]
$$

Upper large intestine (ULI):

$$
q_{U L}(t)=\lambda_{S T} \lambda_{S I}\left[\frac{e^{-\lambda_{S T} t}}{\left(\lambda_{S I}^{\prime}-\lambda_{S T}\right)\left(\lambda_{U L}-\lambda_{S T}\right)}+\frac{e^{-\lambda_{S I}^{\prime}}}{\left(\lambda_{S T}-\lambda_{S I}^{\prime}\right)\left(\lambda_{U L I}-\lambda_{S I}^{\prime}\right)}+\frac{e^{-\lambda_{U L} t}}{\left(\lambda_{S T}-\lambda_{U L}\right)\left(\lambda_{S I}^{\prime}-\lambda_{U L I}\right)}\right]
$$

Lower large intestine (LLI):

$$
\begin{aligned}
q_{L U}(t) & =\lambda_{S T} \lambda_{S I} \lambda_{U L} \\
& {\left[\frac{e^{-\lambda_{S T} t}}{\left(\lambda_{S I}^{\prime}-\lambda_{S T}\right)\left(\lambda_{U L}-\lambda_{S T}\right)\left(\lambda_{L L}-\lambda_{S T}\right)}+\frac{e^{-\lambda_{S I}^{\prime} t}}{\left(\lambda_{S T}-\lambda_{S I}^{\prime}\right)\left(\lambda_{U L}-\lambda_{S I}^{\prime}\right)\left(\lambda_{L U}-\lambda_{S I}^{\prime}\right)}\right.} \\
& \left.\frac{e^{-\lambda_{U I} t}}{\left(\lambda_{S T}-\lambda_{U L}\right)\left(\lambda_{S I}^{\prime}-\lambda_{U L}\right)\left(\lambda_{L U}-\lambda_{U L}\right)}+\frac{e^{-\lambda_{L I} t}}{\left(\lambda_{S T}-\lambda_{L L}\right)\left(\lambda_{S I}^{\prime}-\lambda_{L U}\right)\left(\lambda_{U L}-\lambda_{L U}\right)}\right]
\end{aligned}
$$

where $\lambda_{S I}$ is the total removal coefficient of activity from the small iniestine. The total removal 
coefficient is the sum of the removal coefficient to blood, $\lambda_{s}$ [defined in Eq. (39)], and the coefficient describing the transfer of activity from the small intestine to the upper large intestine, $\lambda_{S I}$, that is

$$
\begin{aligned}
\lambda_{S I} & =\lambda_{S I}+\lambda_{S} \\
& =\lambda_{S I}+\frac{\lambda_{S I} f_{1}}{1-f_{1}} \\
& =\frac{\lambda_{S I}}{1-f_{1}} .
\end{aligned}
$$

The fraction of the ingested activity in the GI-tract at time $t, \boldsymbol{R}_{G I}(t)$, is given as

$$
R_{G I}(t)=q_{S T}(t)+q_{S I}(t)+q_{U L L}(t)+q_{L I I}(t) .
$$

The rate at which ingested activity is transferred to blood, $f_{G I-b l o o d}(t)$, is

$$
f_{G I-b l o o d}(t)=\lambda_{s} q_{S I}(t),
$$

where $\lambda_{s}$ as defined in Eq. (39). Ingested activity is transferred to feces at a rate, $f_{G l-f e c e s}(t)$,

$$
f_{C r-f e c}(t)=\lambda_{L U} q_{L U}(t)
$$

where $q_{L u}(t)$ is defined above. All the gastrointestinal tract functions are sums of exponentials.

The gastrointestinal functions were evaluated using the values of $f_{1}$ for the radionuclides as recommended in ICRP Publication 30 (ref. 5). However, if an $f_{1}$ value of 1 was recommended, the functions are based on a value of 0.95 to avoid the division by zero in the expression for $\lambda_{s}$. 


\subsection{DISTRIBUTION AND RETENTION MODELS}

Following an intake by inhalation and ingestion, radionuclides will be translocated to body fluids at a rate determined by the rate constants for the relevant compartments of the respiratory and gastrointestinal tract. Subsequently, the radionuclide may be taken up by the organs and tissues of the body and retained in these regions as discussed in ICRP Publication 30 (ref. 5). The functions, which describe the retention of radionuclides in the organs/tissues of the body, can be expressed as sums of exponentials. These retention functions take into account implicitly all translocation of the radionuclide once it has entered blood.

\subsection{SYSTEMIC EXCRETION MODELS}

Radionuclides are considered to be excreted from the systemic pool via urinary and fecal excretion. For most radionuclides we have used the excretion functions in ICRP Publication 54 (ref. 4). In some instances, for example plutonium and americium, we have adopted functions derived from the literature. ${ }^{16,17}$ All the excretion functions are expressed as sums of exponentials.

\subsection{Tabulations of Retention and Excretion Functions}

Section 7 of the report sets forth the algorithms used by the RBD software to evaluate the retention and excretion rates associated with acute and chronic intakes of radionuclides. All parameters defining the functions are contained in the nuclide data files. Two software utilities are provided (from the RBD main menu) to access the nuclide files. One utility lists the content of the files while the other tabulates the retention and excretion as a function of time after an acute intake of the radionuclide. The data in these tabulations are of interest to the health physicist in designing a bioassay program and establishing reference levels within that program. An excerpt of the tabulations for U-238 is shown in Table 3. 
Table 3. Partial Tabulation (PLT file) From Unit Retention/Excretion Plot Utility

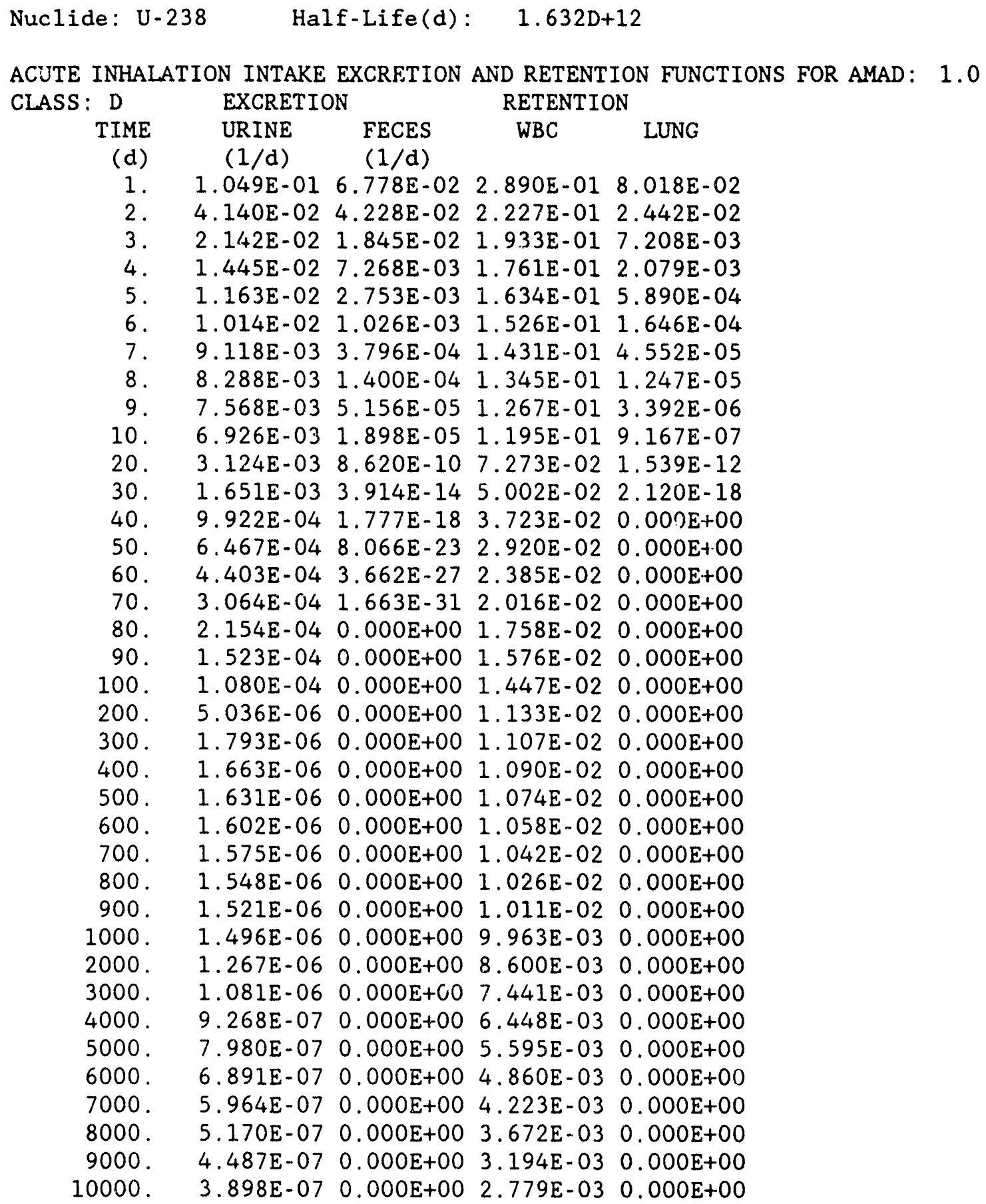




\subsection{CONTENT OF NUCLIDE DATA FILES}

The following briefly describes each set of data within the nuclide files and provides references tor the data presented. The functions describing retention in and translocation from the lung and gastrointestinal tract were derived from the ICRP models as discussed above. An example of the nuclines files, the U-238 file, is given at the end of this section (see Table 4). The user can view and obtain a listing of the contents of any nuclide file using the Nuclide Data LIST Utility from the RBD main menu.

Nuclide Name. Radionuclide name in standard notation (e.g., U-238). Metastable notation can be included. The comment on this line indicates the date of last revision of the nuclide file.

Atomic Mass. The atomic mass of the radionuclide. Data are taken from the 1983 atomic mass evaluation of Wapstra and Audi (ref. 13).

Half-life. The half-life of the radionuclide, in days, as given in ICRP Publication 38 (ref. 14).

Default Route of Intake. If no route of intake is identified in the bioassay record, then this entry provides an assumed route. $K$ corresponds to inhalation, $G$ to ingestion, and $W$ to wound. The default is $H$ (inhalation).

Number of Clearance Classes. The number of clearance classes considered for inhalation of the given radionuclide. As a minimum, the number of clearance classes considered in ICRP Publication 30 (ref. 5) will be included in the file. For each clearance class, functions representing retention in the lung and translocation to blood and to the gastrointestinal tract are specified.

Number of GI-Transfer Functions. The number of different chemical forms considered for ingestion of material (i.e., different $f_{l}$ values). For each form, functions representing retention within the GI tract and translocation to blood are specified.

Number of Systemic Organs. The number of systemic organs for which retention functions are specified. This value is generally zero since lung is specified separately. For radioiodines a value of 1 is assigned, where the thyroid is the organ of interest.

Assign GI-Transfer Function to Inhalation Class (D, W, Y). For each clearance class, the corresponding GI-tract-transfer function is specified. For example, clearance classes $\mathrm{D}$ and $\mathrm{W}$ of uranium use the same GI-tract-transfer functions $\left(f_{l}\right.$ values).

Weights for Results for Clearance Class (D, W, Y). Relative weights to apply when computing the inhalat:on intake if more than one type of assay were carried out. For each clearance class, weights are assigned to breath, urinary, and fecal assays, and to in vivo measurements of whole body and lung. If a systemic organ is specified, a weight is assigned to the assay associated with that organ (e.g., in vivo measurements of thyroid for radioiodine). The weights are based on expert judgement and become important only if more than one assay were performed. 
Weights for Results for Ingestion Class. Relative weights to apply when computing the ingestion intake if more than one type of assay were carried out.

Assign Classes $T, \mathbf{N}$, or $\mathbf{U}$ to $\mathbf{D}, \mathbf{W}$, or $\mathbf{Y}$. Default old clearance class notation to current clearance classes.

Transfer Rate per Day from Lung to Blood. For each clearance class, the parameters of a function describing the rate of translocation of inhaled material from the lung to blood are specified. The data are presented in four columns: the first column gives the rate constants $a_{i}$, the second column shows the coefficients $A_{N P, i}$ for deposition in the $N P$ region, the third column gives the coefficients $\mathrm{A}_{T B_{,} i}$ for deposition in the $T B$ region, and the final column presents the coefficients $A_{P, i}$ for deposition in the $P$ region. The function representing the rate of translocation to blood of inhaled activity $f_{\text {lung }- \text { blood }}(t)$ is

$$
f_{\text {lung }- \text { blood }}(t)=\sum_{i}\left[D_{N P} A_{N P, i}+D_{T B} A_{T B, i}+D_{P} A_{P, i}\right] e^{-a_{i} t},
$$

where $D_{N P}, D_{T B}$, and $D_{P}$ denote the deposition of the aerosol in the $N P, T B$, and $P$ regions of the lung. If an AMAD of $1 \mu \mathrm{m}$ is assumed, then $D_{N P}, D_{T B}$, and $D_{P}$ correspond to $0.30,0.08$, and 0.25 , respectively.

Transfer Rate per Day from Lung to GI Tract. For each clearance class the parameters of the function describing the rate of translocation of inhaled material into the GI tract are specified. The data are presented in four columns: the first column gives the rate constants $a_{i}$, the second column shows the coefficients $A_{N P, i}$ for deposition in the $N P$ region, the third column gives the coefficients $A_{T B, i}$ for deposition in the $T B$ region, and the final column presents the coefficients $A_{P, i}$ for deposition in the $P$ region. The function representing the rate of translocation of inhaled material to blood from the lung $f_{\text {lung } \rightarrow G I}(t)$ is

$$
f_{\text {lung }-G I}(t)=\sum_{i}\left[D_{N P} A_{N P, i}+D_{T B} A_{T B, i}+D_{P} A_{P, i}\right] e^{-a_{i} t}
$$

where $D_{N P}, D_{T B}$, and $D_{P}$ denote the deposition of the aerosol in the $N P, T B$, and $P$ regions of the lung.

Transfer Rate per Day of Ingested Activity to Blood. For each chemical form, the parameters of the function describing the rate of translocation of ingested material to biood are given. The data are presented in two columns: the first column gives the rate constants $a_{i}$, and the second column shows the coefficients $A_{i}$. The function representing the rate of translocation to blood from the GI tract $f_{G I-b l o o d}(t)$ is 


$$
f_{G I-b l o o d}(t)=\sum_{i} A_{i} e^{-a_{i} t}
$$

Transfer Rate per Day of Ingested Activity to Feces. For each chemical form the parameters of the function describing the rate of translocation of ingested material to feces are specified. The data are presented in two columns: the first column gives the rate constants $a_{i}$; the second column shows the coefficients $A_{i}$. The function representing the rate of translocation of ingested material to feces $f_{G l-\text { feces }}(t)$ is

$$
f_{G I-\text { feces }}(t)=\sum_{i} A_{i} e^{-a_{i} t}
$$

Transfer Rate Per Day from Wound Site to Blood. Transfer rate from the would site corresponds to a half-time of 0.25 day.

Urinary Excretion Function. The nuclide file contains the parameters of the function representing the rate of urinary excretion of systemic activity following a unit input into blood at time zero. The data are presented in two columns: the first column gives the rate constants $a_{i}$, and the second column, the corresponding coefficients $A_{i}$. The function representing the urinary excretion $f_{u}(t)$ is thus

$$
f_{u}(t)=\sum_{i} A_{i} e^{-a_{i} t}
$$

For most radionuclides the urinary excretion function is that given in ICRP Publication 54 (ref. 4).

Fecal Excretion Function. The nuclide file contains the parameters of the function representing the rate of fecal excretion of systemic activity following a unit input into blood at time zero. The data are presented in two columns: the first column gives the rate constants $a_{i}$, and the second column, the corresponding coefficients $A_{i}$. The function representing the fecal excretion $f_{f}(t)$ is thus

$$
f_{f}(t)=\sum_{i} A_{i} e^{-a_{i} t}
$$

For most radionuclides the fecal excretion functions used are those in ICRP Publication 54 (ref. 4).

Retention of Inhaled Activity within the Lungs. For each clearance class the parameters of the function describing the retention in the lungs of inhaled material are given. The data are presented in four columns: the first column gives the rate constants $a_{i}$, the second column shows the coefficients $A_{N P, i}$ for deposition in the NP region, the third column gives the coefficients $A_{T B, i}$ for deposition in the $T B$ region, and the final column presents the coefficients $A_{P, i}$ for deposition in the $P$ region. The function representing the retention in the lungs, $R_{\text {lung }}(t)$, of inhaled material is 


$$
R_{\text {lung }}(t)=\sum_{i}\left[D_{N P} A_{N P, i}+D_{T B} A_{T B, i}+D_{P} A_{P, i}\right] e^{-a_{i} t}
$$

where $D_{N P}, D_{T B}$, and $D_{P}$ denote the deposition of the aerosol in the $N P, T B$, and $P$ regions of the lung.

Retention of Ingested Activity within the GI Tract. The nuclide file contains the parameters of the function representing the retention of ingested activity in the tract following a unit input at time zero. The data are presented in two columns: the first column gives the rate constants $a_{i}$, and the second column, the corresponding coefficients $A_{i}$. The retention function $R_{G I}(t)$ is

$$
R_{G I}(t)=\sum_{i} A_{i} e^{-a_{i} t}
$$

The parameters are derived from the GI-Tract model of ICRP Publication 30 (ref. 5).

Retention of Systemic Actrity. The nuclide file contains the parameters of the function representing the retention of a unit input into the systemic pool at time zero. The data are presented in two columns: the first column gives the rate constants $a_{i}$, and the second column, the corresponding coefficients $A_{i}$. The function representing the systemic retention $R(t)$ is thus

$$
R(t)=\sum_{i} A_{i} e^{-a_{i} t}
$$

The parameters of the retention functions are derived from the biokinetic models of ICRP Publications 30 (ref. 5) and 54 (ref. 4).

Retention at Wound Site. Retention at the wound site corresponds to a half-time of 0.25 day.

H/D Dose per Unit Deposition (Sv/Bq) for Inhaled Activity. For each clearance class the nuclide file contains the committed dose equivalent in tissue $T$ per unit deposition in the three regions of the respiratory tract. The data are presented in three columns: the first column gives the coefficients $H_{N P, T}$ for deposition in the NP region, the second column shows the coefficients $H_{T B, T}$ for deposition in the $T B$ region, and the third column presents the coefficients $H_{P, T}$ for deposition in the $P$ region. The committed dose equivalent per unit intake $H_{T}$ for tissue $T$ is given as

$$
H_{T}=D_{N P} H_{N P, T}+D_{T B} H_{T B, T}+D_{P} H_{P, T},
$$

where $D_{N P}, D_{T B}$, and $D_{P}$ denote the deposition of the aerosol in the $N P, T B$, and $P$ regions of the lung. For an $A M A D$ of $1 \mu \mathrm{m}$ the depositions are $0.30,0.08$, and 0.25 , respectively. 
The dosimetric data in the nuclide files were compiled from NUREG/CR-1962 (ref. 15), which is a compilation of data for selected radionuclides developed during completion of ICRP Publication 30 [see also Federal Guidance Report ll (ref. 10)]. In NUREG/CR-1962 values of $H_{T}$ were tabulated for an $\mathrm{AMAD}$ of $1 \mu \mathrm{m}$ with a triplet of numbers $\left(F_{N P}, F_{T B}, F_{P}\right)$ representing the fraction of $H_{T}$ due to deposition in the three regions of the lung. Values of $H_{N P, T}, H_{T B, T}$, and $H_{P, T}$ were computed for the nuclide files as

$$
H_{r, T}=\frac{F_{r}}{D_{r}} H_{T}, \quad r=N P, T B, \text { or } P \text {, }
$$

where $D_{r}$ is the deposition of the aerosol in the region $r$ of the lung model. For an aersol with an AMAD of $1 \mu \mathrm{m}$ the values of $D_{r}$ are $0.30,0.08$, and 0.25 for the $N P, T B$, and $P$ regions, respectively. The dosimetric data presented here is consistent with all four parts of ICRP Publication 30 (ref. 5).

H/D Dose per Unit Intake (Sv/Bq) for Ingested Activity. For each ingestion class the committed dose equivalent per unit intake is tabulated. The data are from NUREG/CR-1962 (ref. 15); see also Federal Guidance Report 11 (ref. 10).

ALI (Bq) from ICRP-30. For each inhalation and ingestion class the Annual Limit on Intake is tabulated from ICRP Publication 30 (ref. 5). 
Table 4. U-238 Nuclide Data File

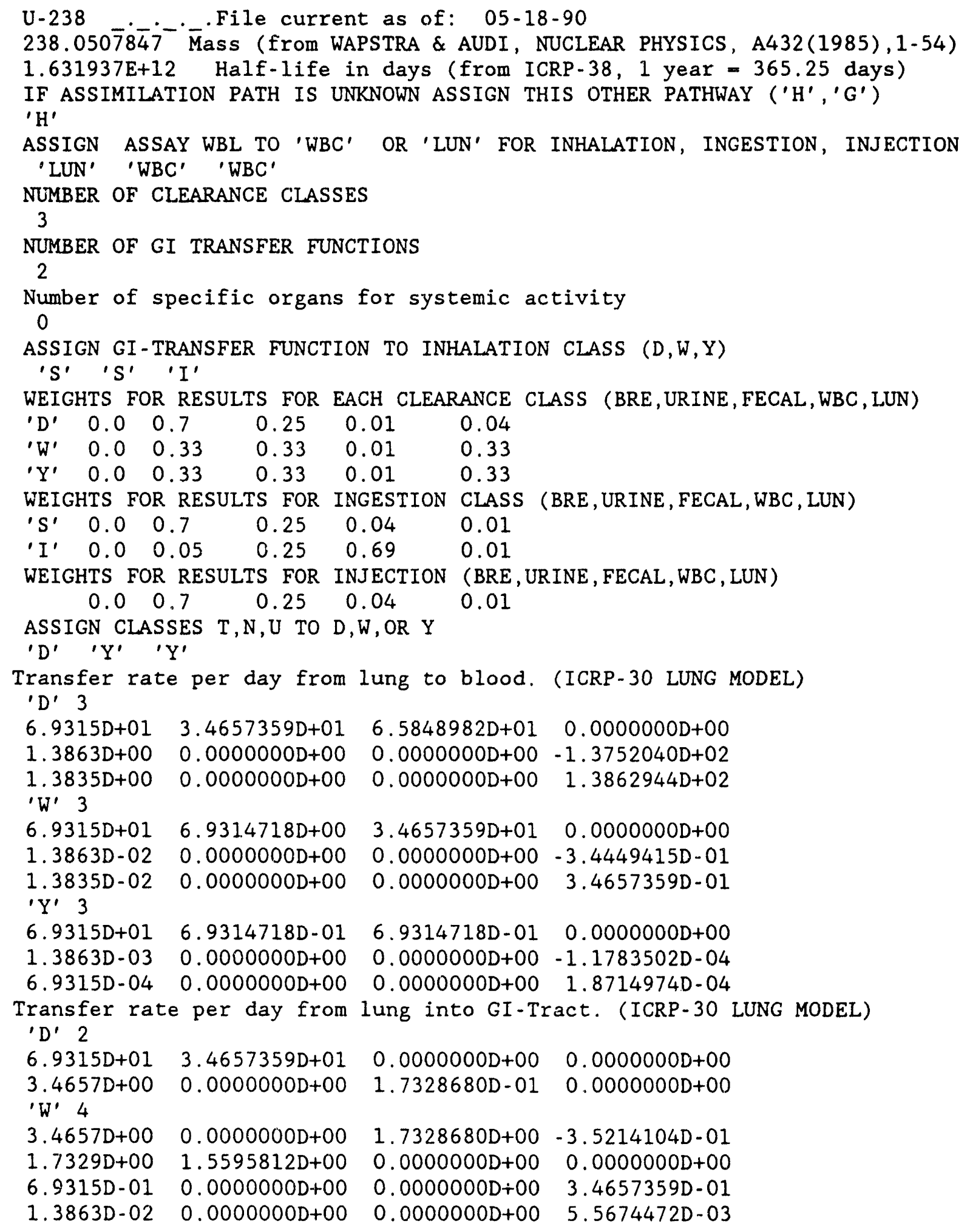




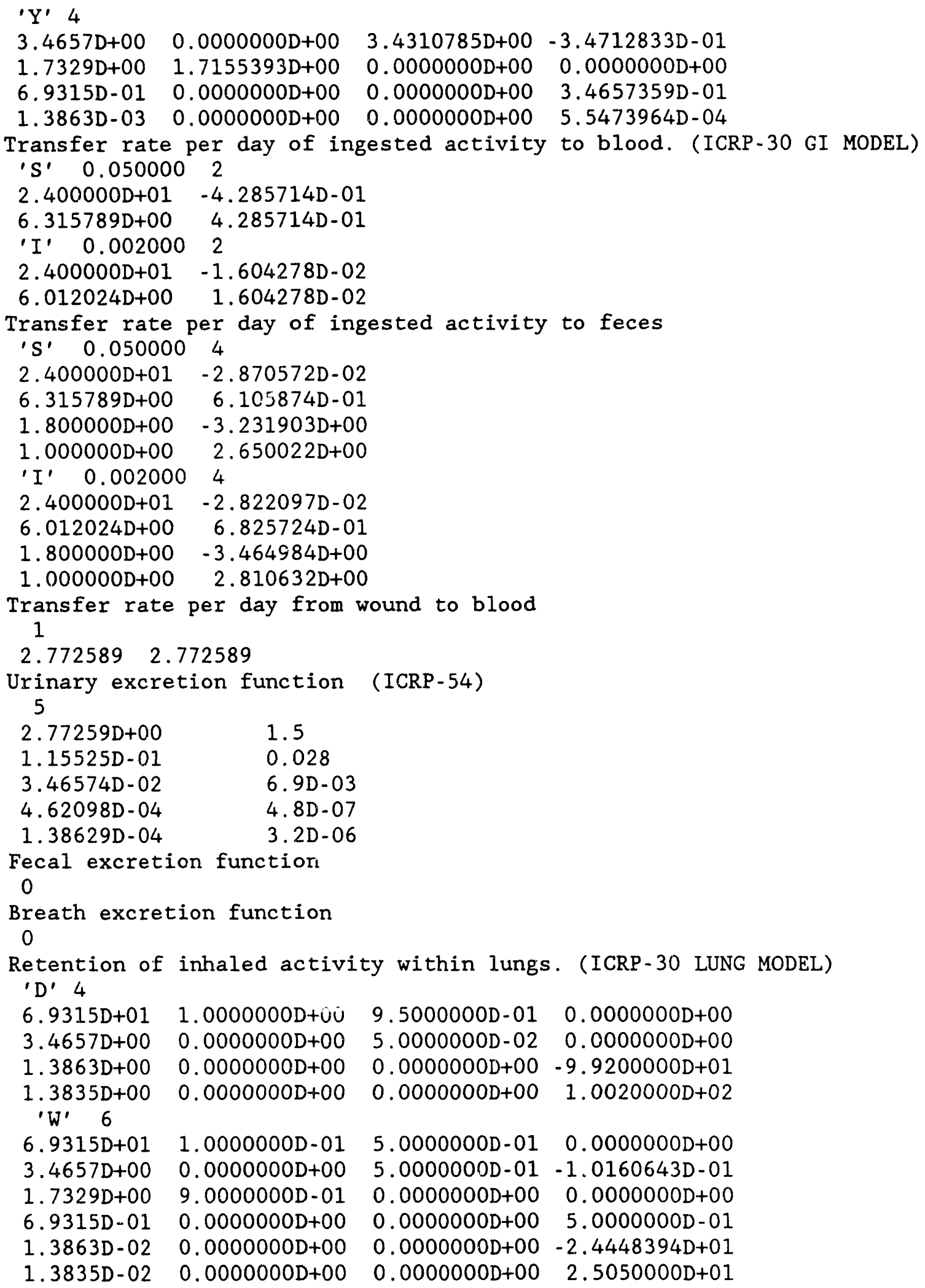




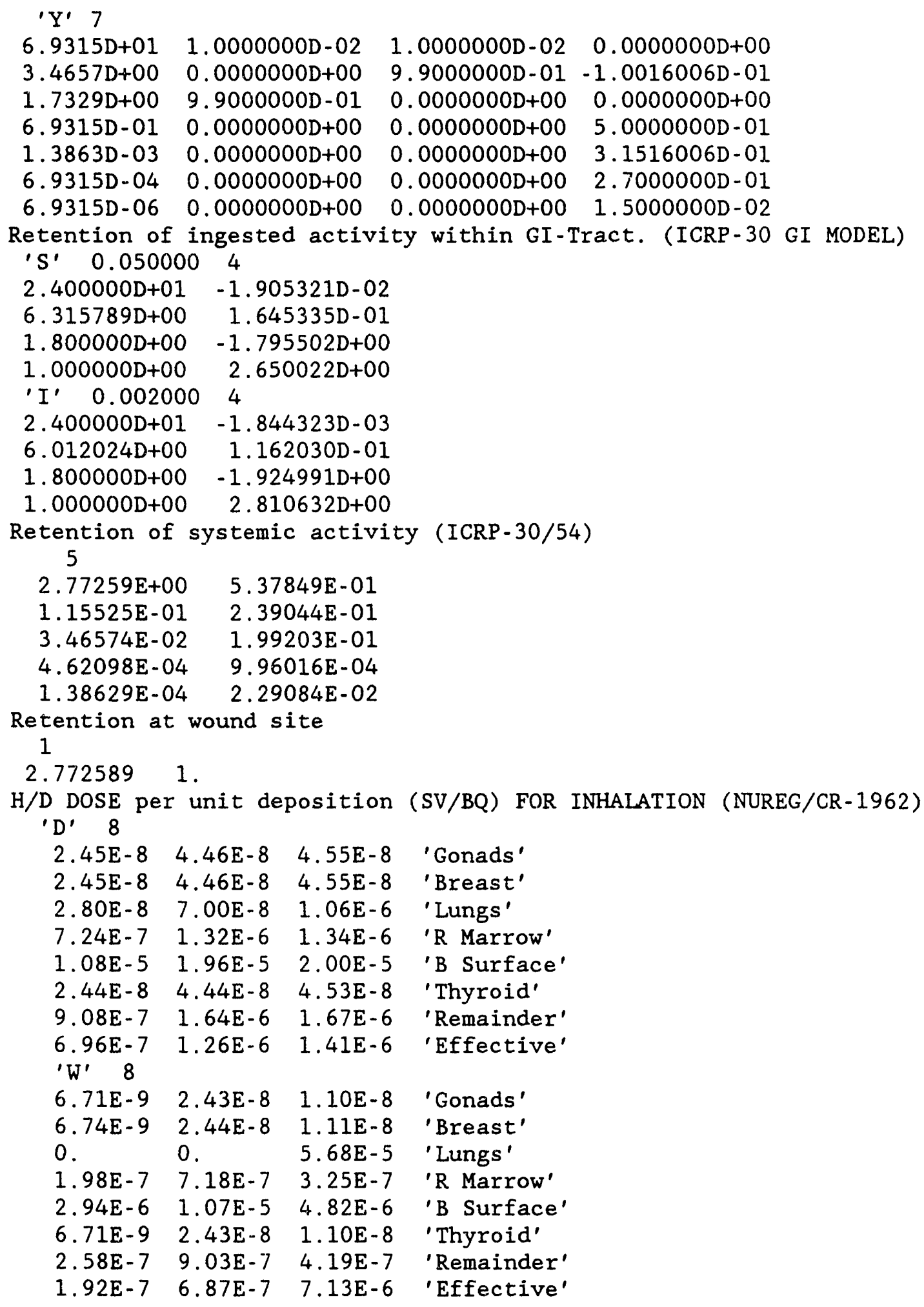


'Y' 8

5.65E-10 6.05E-10 8.81E-9 'Gonads'

5.82E-10 7.28E-10 1.07E-8 'Breast'

$0 . \quad 0.06 \mathrm{E}-3$ 'Lungs'

1.61E-8 1.72E-8 2.50E-7 'R Marrow'

2.36E-7 2.53E-7 3.68E-6 'B Surface'

5.46E-10 6.83E-10 1.00E-8 'Thyroid'

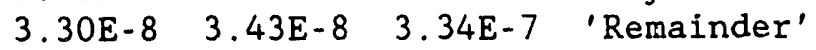

1.91E-8 2.02E-8 1.28E-4 'Effective'

H/I DOSE/INTAKE (SV/BQ) FOR INGESTION, F1=0.05\&0.002 (NUREG/CR-1962)

' $S$ ' $0.050 \quad 8$

2.31E-09 GONAD

2.31E-09 BREAST

2.30E-09 IUNG

6.80E-08 R. MARROW

1.01E-06 BONE SURFACE

2.30E-09 THYROID

$9.69 \mathrm{E}-08$ REMAINDER

6.88E-08 EFFECTIVE

'I' $0.002 \quad 8$

1.02E-10 GONAD

9.33E-11 BREAST

9.22E-11 LUNG

2.72E-09 R. MARROW

4.04E-08 BONE SURFACE

9.20E-11 THYROID

1.61E-08 REMAINDER

6.42E-09 EFFECTIVE

$\operatorname{ALI}(\mathrm{Bq})$ (from ICRP-30)

Inhalation

'D' $5.0 \mathrm{E}+4$

' $W$ ' $3.0 \mathrm{E}+4$

' $Y$ ' $2.0 \mathrm{E}+3$

Ingestion

'S' $0.05 \quad 5.0 \mathrm{E}+5$

'I' $0.0028 .0 \mathrm{E}+6$

FLAG INDICATING PRESENCE (1) OR ABSENCE (0) OF DOSE-RATE FILE FOR NUCLIDE 1 


\section{REFERENCES}

1. NRC (1991). Nuclear Regulartory Commission, 10 CFR Part 20, et al. Standards for Protection Against Radiation; Final Rule, Federal Register 56, No. 98, 23360.

2. International Commission on Radiological Protection, Evaluation of Radiation Dose to Body Tissues from Internal Contamination Due to Occupational Exposure, ICRP Publication 10, Pergammon Press, Oxford, 1968.

3. International Commission on Radiological Protection, The Assessment of Internal Contamination Resulting from Recurrent or Prolonged Uptakes, ICRP Publication 10A, Pergammon Press, Oxford, 1971.

4. International Commission on Radiological Protection, "Individual Monitoring for Intakes of Radionuclides by Workers: Design and Interpretation," Publication 54, Annals of the ICRP, Vol. 19, 1988.

5. International Commission on Radiological Protection, "Limits for Intakes of Radionuclides by Workers," Publication 30, Annals of the ICRP, Vol. 2, 1979.

6. National Council on Radiation Protection and Measurement, Use of Bioassay Procedures for Assessment of Internal Radionuclide Deposition, NCRP Report No. 87, 1987.

7. R. C. Ward and K. F. Eckerman, DOSEXPRT - A Bioassay Dosimetry Code for Martin Marietta Energy Systems, Inc., ORNL/TM-11857, April 1992.

8. E. T. Lessard et al., Interpretation of Bioassay Measurements, NUREG/CR-4884, 1987.

9. L. A. Currie, "Limits for Qualitative Detection and Quantitative Determination: Application to Radio Chemistry," Anal. Chem. 40, 586-593 (1968).

10. K. F. Eckerman, A. B. Wolbarst, and A. C. B. Richardson, Limiting Values of Radionuclide Intake and Air Concentration and Dose Conversion Factors for Inhalation, Submersion, and Ingestion - Federal Guidance Report No. 11, EPA-520/1-88-020, 1988.

11. International Commission on Radiological Protection, "Age-dependent Doses to Members of the Public from Intake of Radionuclides: Part 1," Publication 56, Annals of the ICRP, Vol. 20, No. 2, 1989.

12. G. G. Killough and K. F. Eckerman, "A Conversational Eigenanalysis Program for Solving Differential Equations," In Computer Applications in Health Physics (Eds. R. L. Kathren, D. P. Hicgby, and M. A. McKinney) Proceedings of the 17th Midyear Topical Symposium of the Health Physics Society, 1984. 
13. A. H. Wapstra and G. Audi, "The 1983 Atomic Mass Evaluation," Nucl. Phy. A432, 1985. International Commission on Radiological Protection, Publication 38, "Radionuclide Transformations - Energy and Intensity of Emission," Annals of the ICRP, Vol. 11-13, 1983.

14. International Commission on Radiological Protection, Publication 38, "Radionuclide Transformations - Energy and Intensity of Emission," Annals of the ICRP, Vol. 11-13, 1983.

15. K. F. Eckerman, M. R. Ford, and S. B. Watson, "Internal Dosimetry Data and Methods of ICRP - Part 2," Vol. 1: Committed Dose Equivalent and Secondary Limits, ORNL/NUREG1962/TM-433/V1, 1981.

16. R. W. Leggett and K. F. Eckerman, "A method for estimating the systemic burden of Pu from urinalysis," Health Phys. 52(13), 337-346 (1987).

17. R. W. Leggett, "A retention-excretion model for americium in humans," Health Phys. 62(4), 288-310 (1992). 


\section{APPENDIX A FORMAT OF BIOASSAY RECORDS}

This appendix contains the format for bioassay records (DAT) files written by RBDBASE and read by RBDCOMP. Each record is 230 bytes long. The templates are identical for in vivo and in vitro assays for all variables up to the incident time (column 70), because these variables are used in sorting the records. Beyond that (columns 71-141) the records differ only in the presence of the assay sample volume (or mass) and excretion rate for in vitro assays. Columns 142-230 contain the individual's name, the bioassay data base file name (DBF file) from which the records were extracted, the date of creation of the bioassay records (DAT) file, and a comment. The example input shown here is from an I-131 incident with urinalysis and thyroid counts (DEMOAIOD.DAT) and lung counts following an inhalation exposure to mixed-class aerosol of U-238.

REASON code indicates incident (1) or routine (3) exposure. The in vitro assays allowed in RBD are breath (BRE), urine (U), or feces (F). The in vivo assays allowed are whole body count (WBC), lung count (LUN), or thyroid count (THY). The pathways allowed in RBD are inhalation (H), ingestion $(G)$, and wound $(W)$. Committed doses are not computed for wound pathway; only the intake is computed.

The lung clearance classes allowed for inhalation are day (D), week (W), year (Y), vapor (V) [e.g., tritium], mixed class (M) [fractional mixture of $D, W$, and $Y$ ], and labeled organic compound (L) which is used with radiocarbon. If mixed class is chosen, you must also add a mixed-class identifier (0-9) making the class designation M0, for example, and you must supply the percentage of $\mathrm{D}, \mathrm{W}$, and $\mathrm{Y}$ in the mixture. The classes allowed for ingestion are very insoluble (V), insoluble (I), and soluble (S).

The mixed-class percentage field contains, for the allowed inhalation classes, the class designation followed by the percentage for that class. Only allowed inhalation classes for the nuclide occupy this field. For example, for U-238, where all three classes (D, W, and Y) exist, if one mixes $20 \%$ class D, $30 \%$ class $\mathrm{W}$, and $50 \%$ class $\mathrm{Y}$, the field would read D $20 \mathrm{~W} 30 \mathrm{Y} 50$. However, for Pu-241, where only two inhalation classes ( $\mathrm{W}$ and $\mathrm{Y}$ ) exist, if one mixes $50 \% \mathrm{~W}$ and $50 \% \mathrm{Y}$, the field would read W 50: Y 50, with the remainder of the field left blank. The percentages must sum to 100 . 


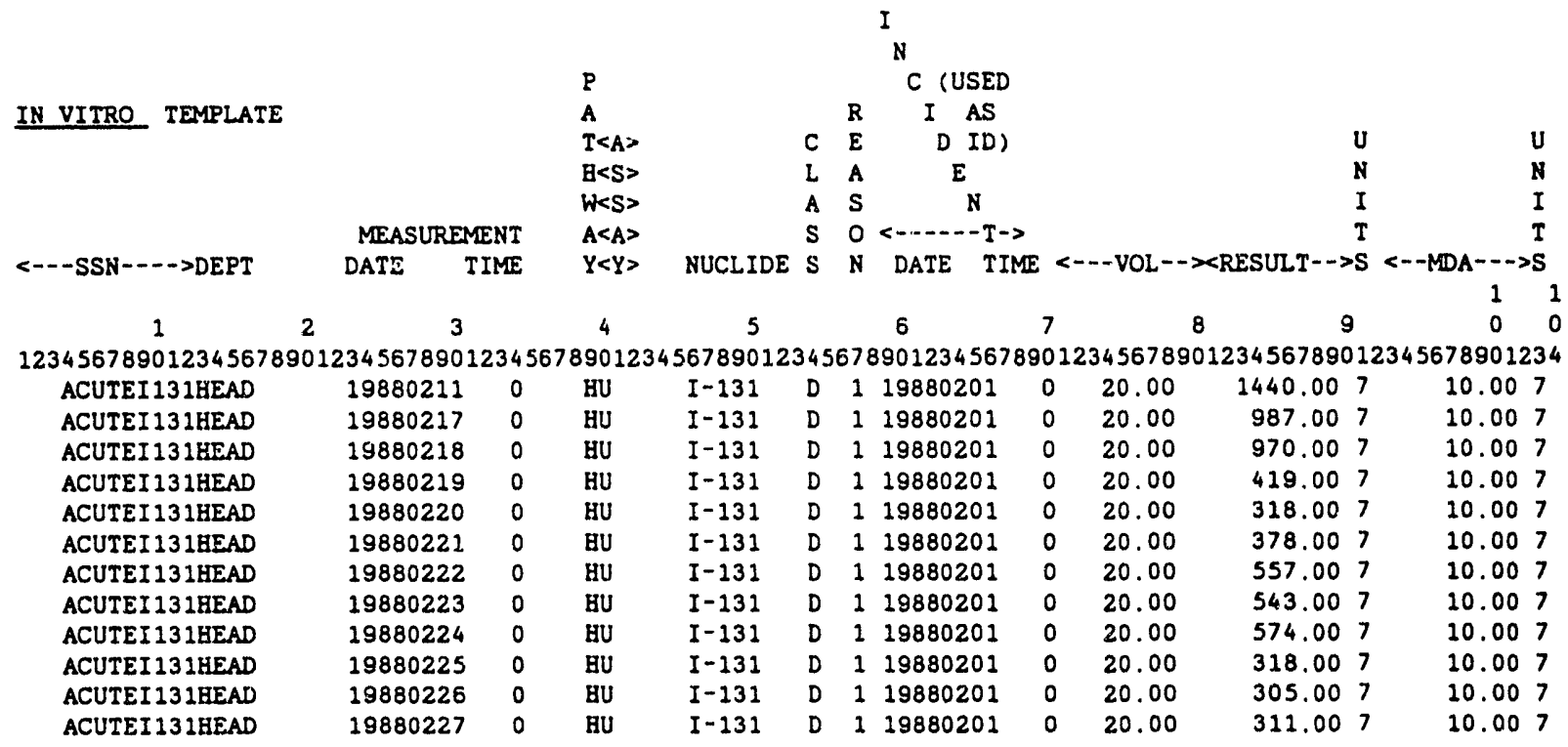

(continued)

$\begin{array}{llll} & \text { C } & \text { C } & \text { C } \\ \text { FOR } & \text { L } & \text { L } & \text { L } \\ \text { MIXED - - - } & \text { A } & \text { A } & \text { A } \\ \text { CLASS } & \text { S } & \text { S } & \text { S } \\ & \text { S } & \text { S } & \text { S }\end{array}$

. DATA BASE. DAT FILE

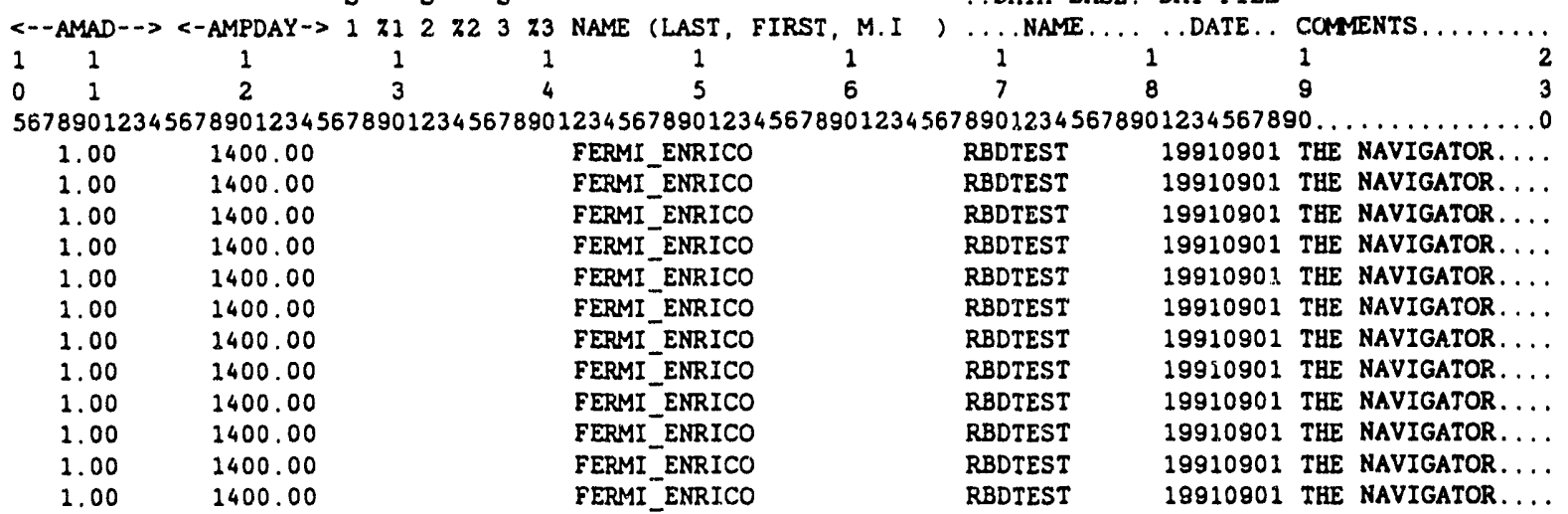




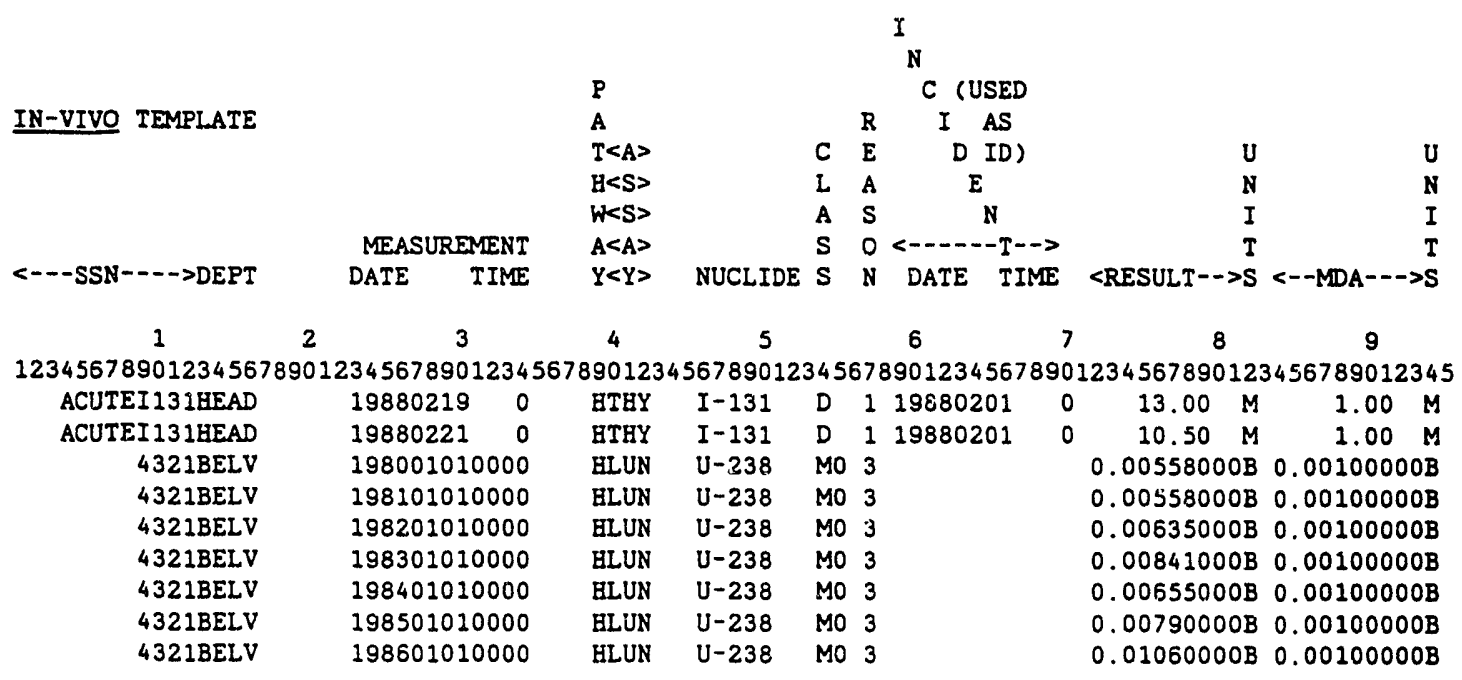

(continued)

$\begin{array}{lrrr} & \text { C } & \text { C } & \text { C } \\ \text { FOR } & \text { L } & \text { L } & \text { L } \\ \text { MIXED } & -\cdots \text { A } & \text { A } & \text { A } \\ \text { CLASS } & \text { S } & \text { S } & \text { S } \\ & \text { S } & \text { S } & \text { S }\end{array}$

<--AMAD--> $12712282 \quad 3 \quad 23$

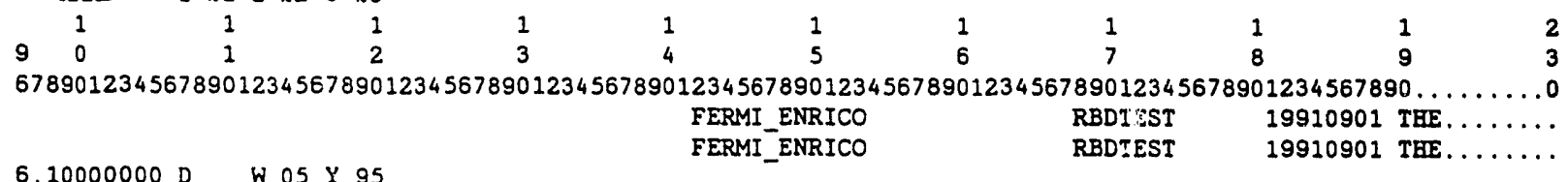
$6.10000000 \mathrm{D}$
W $05 Y$
6. $10000000 \mathrm{D}$
$05 Y 95$
W 05 Y 95
W 05 Y 95
$6.10000000 \mathrm{D}$
W 05 Y 95
W $05 Y 95$
6.10000000
W 05 Y 95
NAME (LAST, FIRST, M.I ) .. NAME ... . . DATE. COMENTS... FERMI_ENRICO RBDTEST


The following is the forms: for the bioassay records (DAT) files used by RBDCOMP. The RBDBASE program creates sorted ASCII records with this format. If two or more DAT files are

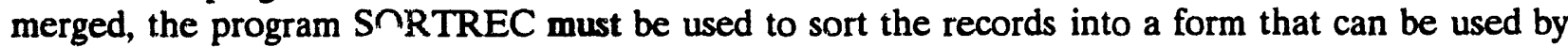
RBDCOMP.

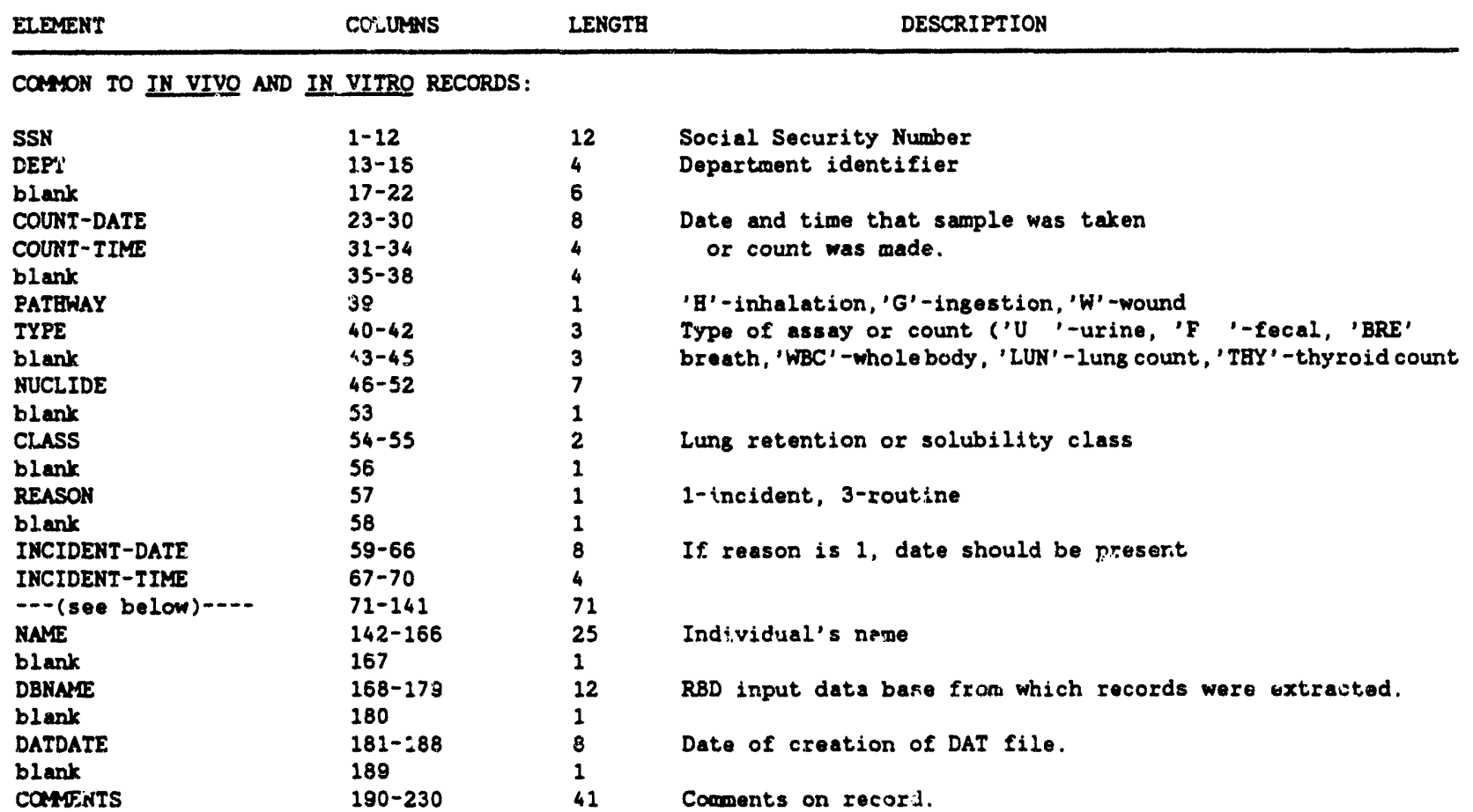

SPECIFIC TO IN VIVO RECORDS:

$\begin{array}{ll}\text { blank } & 71 \\ \text { RESULI } & 72-81 \\ \text { UNITS } & 82 \\ \text { blank } & 83 \\ \text { MDA } & 84-93 \\ \text { M-UNIIS } & 94 \\ \text { blank } & 95 \\ \text { AMAD } & 96-105 \\ \text { blank } & 106 \\ \text { CLASS1 } & 107 \\ \text { blank } & 108 \\ \text { PERCEHI1 } & 109-110 \\ \text { blank } & 111 \\ \text { CLASS2 } & 112 \\ \text { blank } & 113 \\ \text { PERCENT2 } & 114-115 \\ \text { blank } & 126 \\ \text { CLASS3 } & 117 \\ \text { blank } & 118 \\ \text { PESCENT3 } & 119-120\end{array}$

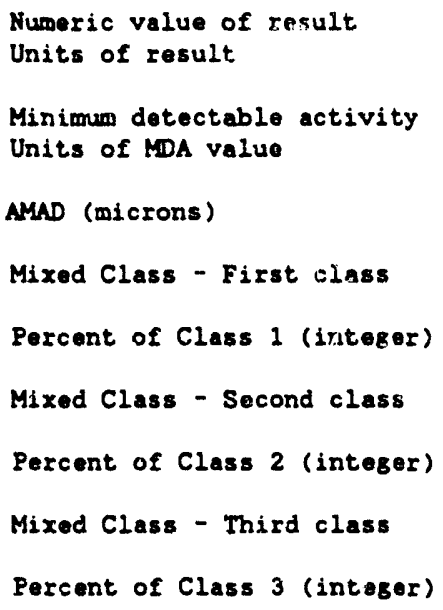


SPECIEIC TO IN VITRO RECORDS:

\begin{tabular}{llll} 
VOL & $71-80$ & 10 & Volume of sample (aliquot) (ml) \\
RESUIT & $81-90$ & 10 & Numeric value of result \\
UNITS & 91 & 1 & Units of result \\
blank & 92 & 1 & \\
MDA & $93-102$ & 10 & Minimum detectable activity \\
M-UNITS & 103 & 1 & Units of MDA value \\
blank & 104 & 1 & \\
AMA & $105-114$ & 10 & AMAD (microns) \\
blank & 115 & 1 & \\
AMPDAY & $116-125$ & 10 & Excretion rate (ml/day) \\
blank & 126 & 1 & \\
CLASS1 & 127 & 1 & Mixed Class - First class \\
blank & 128 & 1 & \\
PERCENT1 & $129-130$ & 2 & Percent of Class 1 (integer) \\
blank & 131 & 1 & \\
CLASS2 & 132 & 1 & Mixed Class - Second class \\
blank & 13 & 1 & \\
PERCENT2 & $134-135$ & 2 & Percent of Class 2 (integer) \\
blank & 136 & 1 & \\
CLASS3 & 137 & 1 & Mixed Class - Third class \\
blank & 138 & 1 & \\
PERCENT3 & $139-140$ & 2 & Percent of Class 3 (integer) \\
\hline
\end{tabular}

DATES are in the form YYYYMMDD, and TIMES are in the form HHMM. For example, a dat $\because$ of Jan 5, 1989, and time of 3:45 PM would be represented as 198901051545.

There are three in vivo assays provided in the program: whole body count (WBC), lung count ( $\mathrm{L} U \mathrm{UN}$ ), and thyroid count (THY). Units for in vivo records are 'M' for $\mu \mathrm{Ci}$, ' $\mathrm{N}$ ' for $\mathrm{nCi}$, ' $\mathrm{P}$ ' for $\mathrm{pCi}$, 'D' for dpm (aisintegrations/minute), ' $U$ ' for $\mu \mathrm{g}$, and 'B' for becquerel.

There are three in vitro assays provided in the program: breath analysis (BRE), urinalysis (U), and fecal analysis ( $\left({ }^{\prime}\right)$. Codes for units in urinalysis records are 1 for $\mathrm{dpm} / \mathrm{ml}, 2$ for dpm/day, 3 for $\mathrm{dpm} / \mathrm{sample}, 4$ for $\mathrm{dpm} / \mathrm{L}, 5$ for $\mathrm{Hg} / \mathrm{ml}, 6$ for $\mathrm{Bq} / \mathrm{L}$, and 7 for $\mathrm{Bq} /$ day. Codes for units for fecal analysis records are 3 for dpn/sampie and 7 for $\mathrm{Bq} /$ day. Codes for units for breath anaylsis records are 2 for $\mathrm{dpm} /$ day, 3 for dpm/sample, 4 for dpm/L, 6 for $\mathrm{Bq} / \mathrm{L}$, and 7 for $\mathrm{Bq} / \mathrm{day}$.

To be recognized by RBD, the social security number (SSN) must be in the same position on each record and must be of a consistent format. SSN is a character variable, allowing alphabetic as well as numeric values. The dash (or hyphen) character is allowed; for example, 000-00-0000 is an allowable SSN. You should create your own convention for SSN.

RBD bioassay exposure history records contain the fields that are common to in vivo and in vitro records (columns 1-70), with the exceptions that COUNT-DATE and COUNT-TIME are date and time of start or end of exposure and that the assay code (TYPE) is AAA for start of exposure and $\mathrm{ZZZ}$ for end of exposure. 


\section{APPENDIX B. FORMAT OF OUTPUT RECORDS}

This appendix contains the format for output records (DBS) files written by RBDCOMP. Each output record is 313 bytes long. The example output file shown here is DEMOAIOD.DBS, an I-131 incident with urinalysis and in vivo thyroid measurements, discussed in the tutorial.

REASON code designates incident (1) or routine (3) exposure. The in vitro assays allowed in RBD are breath (BRE), urine (U), or feces (F). The in vivo assays allowed are whole body count (WBC), lung count (LUN), or thyroid count (THY). Pathways allowed in RBD are inhalation (H), ingestion (G), and wound (W). Committed doses are not computed for wound pathway; only the intake is computed.

The classes allowed for inhalation are day (D), week (W), year (Y), vapor (V) [e.g. tritium], mixed class (M), and labeled organic compound (L), which is used with radiocarbon. If mixed class is chosen, you must also add a mixed-class identifier (0-9), making the class designation M0, for example, and you must supply the percentage of $\mathrm{D}, \mathrm{W}$, and $\mathrm{Y}$ in the mixture. The classes allowed for ingestion are very insoluble (V), insoluble (I), and soluble (S).

The mixed-class percentage field contains, for the allowed inhalation classes, the class designation followed by the percentage for that class. Only allowed inhalation classes for the nuclide occupy this field. For example, for U-238, where all three classes (D, W, and Y) exist, if one mixes $20 \%$ class D, $30 \%$ class W, and 50\% class Y, the field would read D 20 W 30 Y 50. However, for Pu-241, where only two inhalation classes (W and $\mathrm{Y}$ ) exist, if one mixes $50 \% \mathrm{~W}$ and $50 \% \mathrm{Y}$, the field would read W 50 Y 50, with the remainder of the field left blank. The percentages must sum to 100 . 

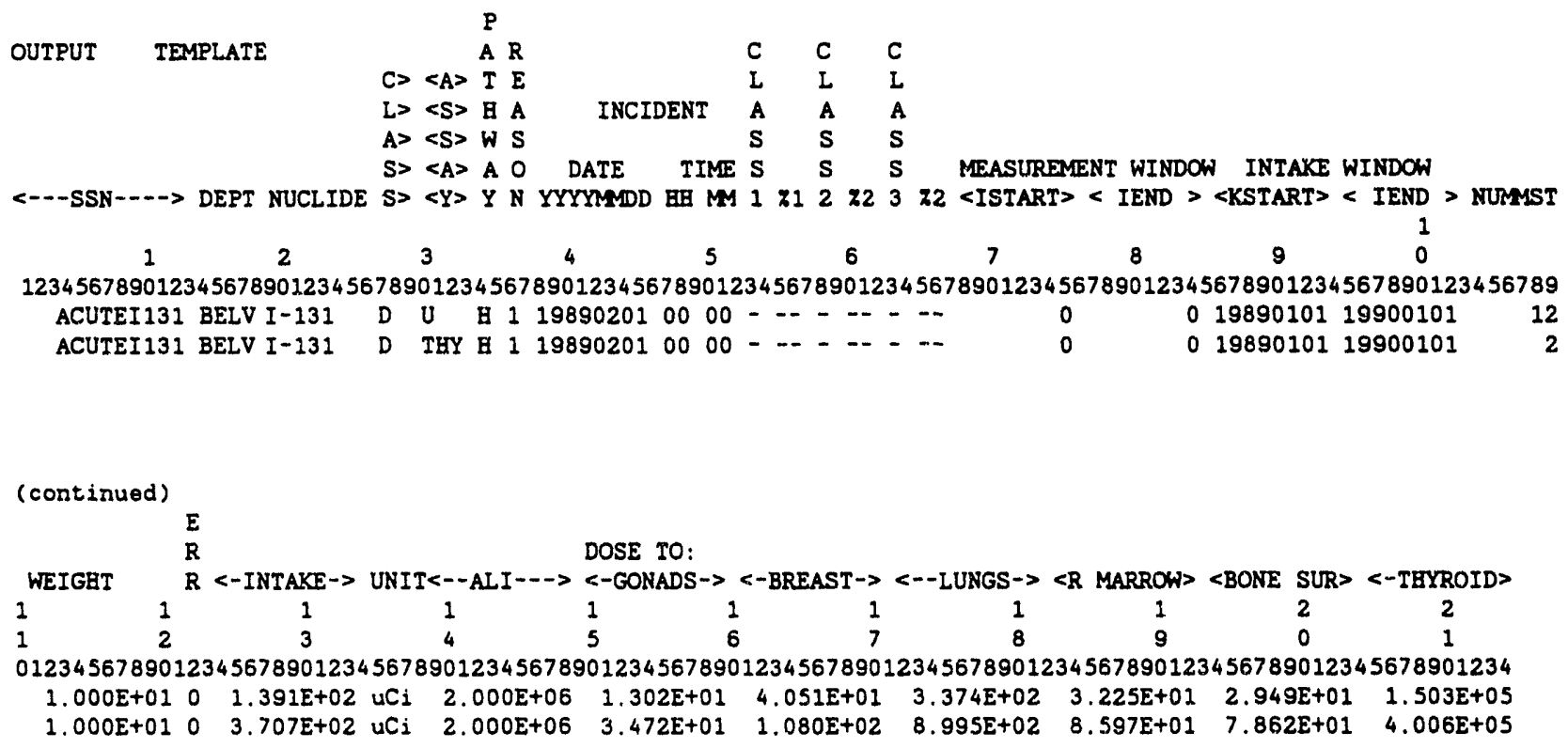

\begin{tabular}{|c|c|c|c|c|c|c|c|c|c|c|c|c|}
\hline & 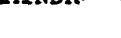 & 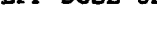 & $Y$ & YYMMDD H & $\mathrm{HH}$ MM & NAME LLAST, & FIRST, M.I. & ) & . . NAME. . . & . DATE. & NUC & DATE \\
\hline 2 & 2 & 2 & 2 & & 2 & 2 & 2 & 2 & 2 & 3 & & 33 \\
\hline 1 & 2 & 3 & 4 & & 5 & 6 & 7 & 8 & 9 & 0 & & 1 \\
\hline & 390123456 & 7890123456 & 789012 & 234567890 & 0125 & 56789012345 & 56789012345678 & 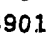 & 89012 & 56789012 & & 20123 \\
\hline & $141 E+01$ & $4.573 E+03$ & mrem & 19910901 & 113 & 29 FERMI_EN & TRICO & & RBDTEST & 19910901 & $05-1$ & $18-90$ \\
\hline & $104 E+02$ & $1.219 \mathrm{E}+\mathrm{C} 4$ & mrem & 19910901 & 113 & 29 FERMI EN & NRICO & & RBDTEST & 19910901 & $05-1$ & $18-90$ \\
\hline
\end{tabular}


The following is the format for the RBD output records. Output records files can be loaded into an output data base (for example: OUTTEST.DBF), and output records can be keyed to the bioassay records used.

\begin{tabular}{|c|c|c|c|}
\hline ELDMENT & COLUMNS & LENGTB & DESCRIPTION \\
\hline SSN & $1-12$ & 12 & Social Security Number \\
\hline blank & 13 & 1 & \\
\hline DEPT & $14-17$ & 4 & Department identifier \\
\hline blank & 18 & 1 & \\
\hline NUCLIDE & $19-25$ & 7 & Nuclide \\
\hline blank & 26 & 1 & \\
\hline CLASS & $27-28$ & 1 & Lung retention or solubility class \\
\hline blank & 29 & 1 & \\
\hline TYPE & $30-32$ & 3 & Type of assay or count ('U '-urine, 'F '-fecal, 'BRE' \\
\hline blank & 33 & 1 & breath, 'WBC'-whole body, 'LUN'-lung count, 'THY'-thyroid count) \\
\hline PATHWAY & 34 & 1 & 'B'-inhalation, 'G'-ingestion, 'W'-wound \\
\hline blank & 35 & 1 & \\
\hline REASON & 36 & 1 & 1-incident, 3-routine \\
\hline blank & 37 & 1 & \\
\hline IDATE & $38-51$ & 14 & Incident date [YYYYMMD] and time [HH MM] \\
\hline blank & 52 & 1 & \\
\hline CLASS 1 & 53 & 1 & Mixed Class - First class \\
\hline blank & 54 & 1 & \\
\hline PERCENT 1 & $55-56$ & 2 & Percent of Class 1 (integer) \\
\hline blank & 57 & 1 & \\
\hline CLASS2 & 58 & 1 & Mixed Class - Second class \\
\hline blank & 59 & 1 & \\
\hline PERCENT2 & $60-61$ & 2 & Percent of Class 2 (integer) \\
\hline blank & 62 & 1 & \\
\hline CLASS3 & 63 & 1 & Mixed Class - Third class \\
\hline blank & 64 & 1 & \\
\hline PERCENT3 & $65-66$ & 2 & Percent of Class 3 (integer) \\
\hline blank & 67 & 1 & \\
\hline ISTART & $68-75$ & 8 & Start date [YYYMMDD] for routine calculations \\
\hline blank & 76 & 1 & \\
\hline IEND & $77-84$ & 8 & End date [YYYYMDD] for routine calculations \\
\hline blank & 85 & 1 & \\
\hline KSTART & $86-93$ & 8 & Start date [YYYYMMD] for intake calculations \\
\hline blank & 94 & 1 & \\
\hline KEND & $95-102$ & 8 & End date [YYYYMMD] for intake calculations \\
\hline blank & 103 & 1 & \\
\hline NUMMST & $104-109$ & 6 & Number of measurements (or -1 ) \\
\hline blank & 110 & 1 & \\
\hline WEIGHT & $111-120$ & 10 & Assay weight (or total weight) \\
\hline blank & 121 & 1 & \\
\hline IERR & 122 & 1 & Error code ( 0 - no error, 1 - window error, 2,3 - record error) \\
\hline blank & 123 & 1 & \\
\hline INTAKE & $124-133$ & 10 & Intake in micro Curies (uCi) \\
\hline blank & 134 & 1 & \\
\hline INTUNIT & $135-137$ & 3 & Units of intake in micro Curies ( $u C i$ ) \\
\hline blank & 138 & 1 & \\
\hline ALI & $139-148$ & 10 & ALI in micro Curies ( $u C i$ ) \\
\hline blank & 149 & 1 & \\
\hline DOSE TO: GONADS & $150-159$ & 10 & Dose in mrem \\
\hline blank & 160 & 1 & \\
\hline BREAST & $161-170$ & 10 & Dose in mrem \\
\hline blank & 171 & 1 & \\
\hline LUNG & $172-181$ & 10 & Dose in mrem \\
\hline blank & 182 & 1 & \\
\hline R MARROW & $183-192$ & 10 & Dose in mrem \\
\hline blank & 193 & 1 & \\
\hline BONE SURF & $194-203$ & 10 & Dose in mrem \\
\hline blank & 204 & 1 & \\
\hline TEYROID & $205-214$ & 10 & Dose in mrem \\
\hline
\end{tabular}




\begin{tabular}{llll} 
blank & 215 & 1 & \\
$\quad$ REMAINDER & $216-225$ & 10 & Dose in mrem \\
blank & 226 & 1 & \\
EFFECTIVE DOSE & $227-236$ & 10 & Dose in mrem \\
blank & 237 & 1 & \\
DOSEUNIT & $238-241$ & 4 & Units of dose (mrem) \\
blank & 242 & 1 & \\
DDATE & $243-256$ & 14 & Date [YYYYMDD] and time [HE MM] of computation \\
blank & 257 & 1 & \\
NARE & $258-282$ & 25 & Individual's name \\
blank & 283 & 1 & \\
DBNAME & $284-295$ & 12 & RBD input data base name \\
blank & 296 & 1 & \\
DATDATE & $297-304$ & 8 & Date of creation of DAT file \\
blank & 305 & 1 & \\
NUCDATE & $306-313$ & 8 & Date of Nuclide Data File \\
\hline
\end{tabular}

DATES are in the form YYYYMMDD, and TIMES are in the form HHMM. For example, a date of Jan 5, 1989, and time of 3:45 PM would be represented as 198901051545.

There are three in vivo assays provided in the program: whole body count (WBC), lung count (LUN), and thyroid count (THY). Units for in vivo records are 'M' for $\mu \mathrm{Ci}$, ' $\mathrm{N}$ ' for $\mathrm{nCi}$, 'P' for $\mathrm{pCi}$, ' $D$ ' for dpm (disintegrations/minute), ' $U$ ' for $\mu g$, and ' $B$ ' for becquerel.

There are three in vitro assays provided in the program: breath analysis (BRE), urinalysis $(\mathrm{U})$, and fecal analysis (F). Codes for units for urinalysis records are 1 for $\mathrm{dpm} / \mathrm{ml}, 2$ for dpm/day, 3 for $\mathrm{dpm} / \mathrm{sample}, 4$ for $\mathrm{dpm} / \mathrm{L}, 5$ for $\mu \mathrm{g} / \mathrm{ml}, 6$ for $\mathrm{Bq} / \mathrm{L}$, and 7 for $\mathrm{Bq} /$ day. Codes for units for fecal analysis records are 3 for dpm/sample and 7 for $\mathrm{Bq} /$ day. Codes for units for breath analysis records are 2 for $\mathrm{dpm} / \mathrm{day}, 3$ for $\mathrm{dpm} / \mathrm{sample}, 4$ for dpm/L, 6 for $\mathrm{Bq} / \mathrm{L}$, and 7 for $\mathrm{Bq} / \mathrm{day}$.

To be recognized by RBD, the social security number (SSN) must be in the same position on each record and must be of a consistent format. SSN is a character variable, allowing alphabetic as well as numeric values. The dash (or hyphen) character is allowed; for example, 000-00-0000 is an allowable SSN. You should create your own convention for SSN. 


\section{APPENDIX C. FORMAT OF COMMTTTED EFFECTIVE DOSE RECORDS}

This appendix contains the format for the committed effective dose (CED) records written by the REPORT program. Each record is 120 bytes in length. The example shown here is file $D E M O A I O D . C E D$, an I-131 incident with urinalysis and in vivo thyroid measurements, discussed in the tutorial.

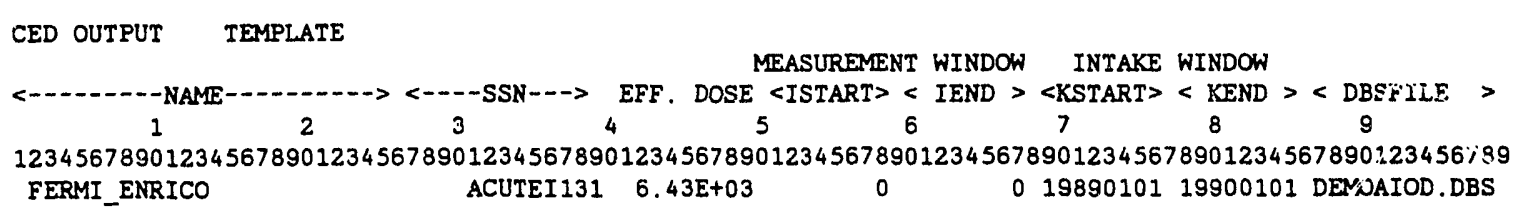

The following is the format for the CED output. CED output files will be loaded into the external data base on the mainframe computer at Redstone Arsenal, Huntsville, Alabama.

\begin{tabular}{llll} 
ELEMENT & COLUMN & LENGTH & DESCRIPTION \\
\hline blank & 1 & 1 & \\
NAME & $2-26$ & 25 & Individual's Name \\
blank & 27 & 1 & \\
SSN & $28-39$ & 12 & Social Security Number \\
blank & 40 & 1 & \\
EFFECTIVE DOSE & $41-49$ & 9 & Dose in mrem \\
blank & 50 & 1 & \\
ISTART & $51-58$ & 8 & Start date [YYYYMDD] for routine calculations \\
blank & 59 & 1 & \\
IEND & $60-67$ & 8 & End date [YYYYMDD] for routine calculations \\
blank & 68 & 1 & \\
KSTART & $69-76$ & 8 & Start date [YYYYMDD] for intake calculations \\
blank & 77 & 1 & \\
KEND & $78-85$ & 8 & End date [YYYYMMD] for intake calculations \\
blank & 86 & 1 & \\
DBSNAME & $87-98$ & 12 & RBD input data base name \\
blank & 99 & 1 & \\
DBNAME & $100-111$ & 12 & RBD input data base name \\
blank & 112 & 1 & \\
DATDATE & $113-120$ & 8 & Date of creation of DAT file
\end{tabular}




\section{APPENDIX D. CONTENTS OF SITEDFT FILE}

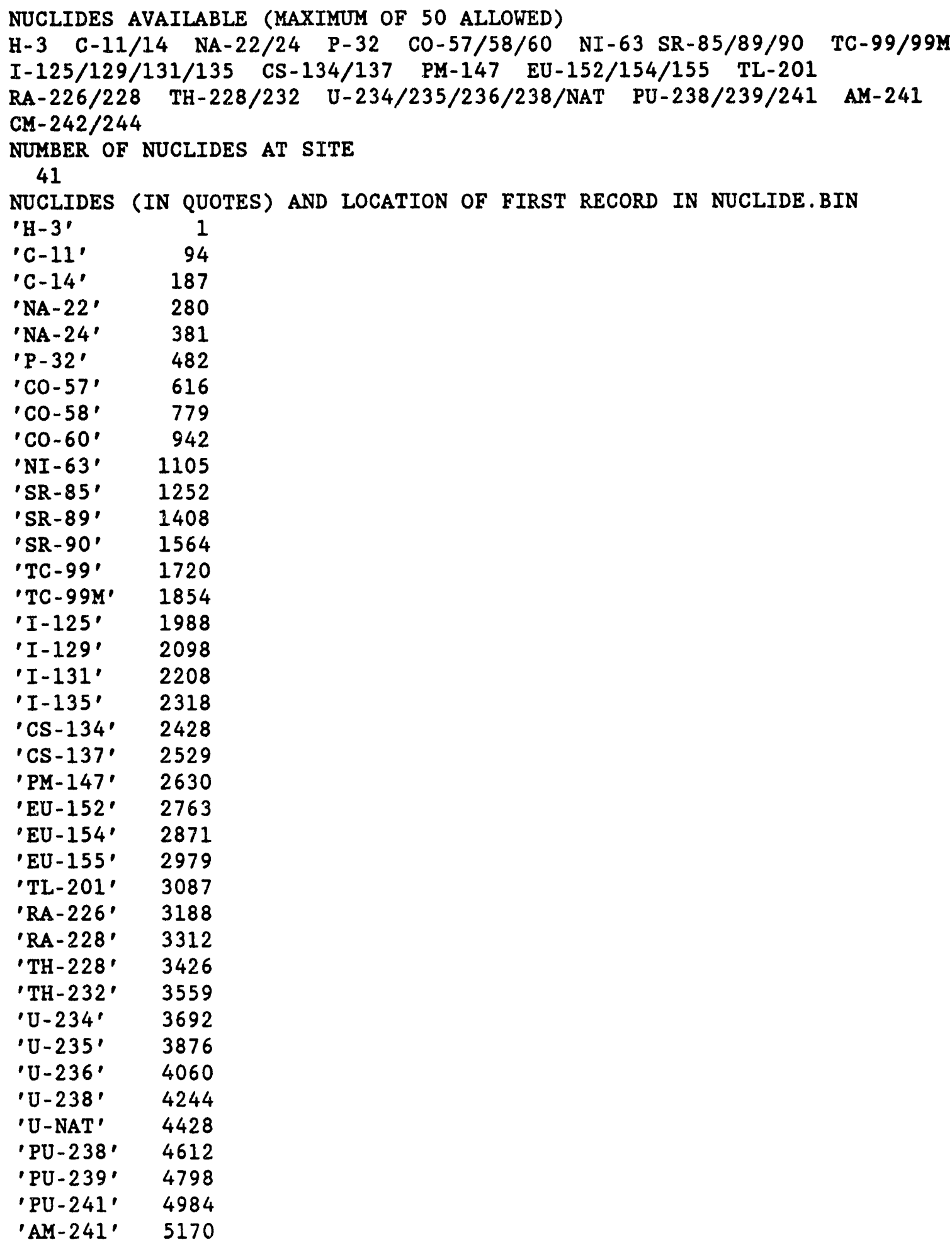




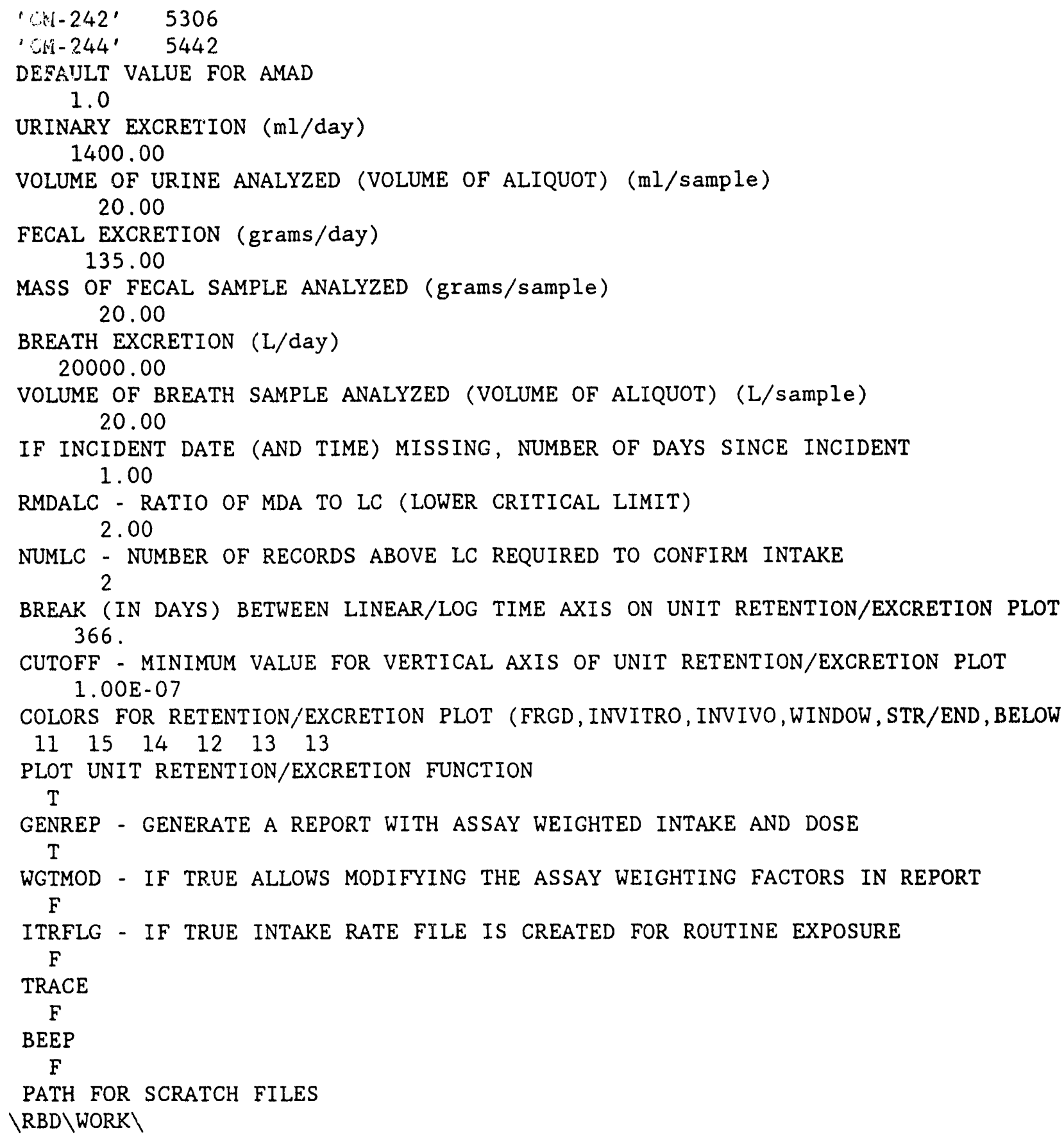




\section{APPENDIX E EASY MODE OF OPERATION OF RBD}

RBD can now be run in either an ADVANCED mode, as described in the body of this report, or an EASY mode, which will be described in this appendix. To set the mode of operation of RBD, type SETUPRBD at the DOS prompt while in the RBD directory. SETUPRBD runs the SETUPRBD program. When SETUPRBD asks for Easy or Advanced Mode?, enter $A$ for ADVANCED mode or $E$ for EASY mode. Below is the SETUPRBD menu as it would appear on using DEMO as the dosimetry account code and on selecting the EASY mode. Note that if the EASY mode is selected, the user must specify the display option for the RBDCOMP program. In EASY mode, this same display option is also used by the REPORT program.

\section{RBD SETUP}

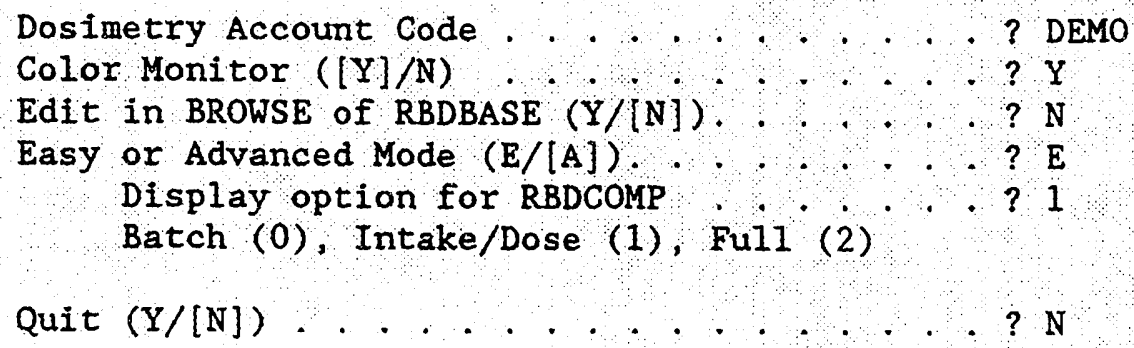

The following comments apply when you have selected the EASY mode. You are restricted to a single bioassay data base and a single output data base. The data bases filenames are constructed by adding the dosimetry account code to RBD and OUT, for the bioassay and output data bases, respectively. Assume, as we did in the tutorial, that the dosimetry account code is $D E M O$. The bioassay data base would be RBDDEMO.DBF, and the output data base would be OUTDEMO.DBF. These data bases are created the first time you select Input Data Base - RBDBASE from the RBD main menu. You must select Input Data Base - RBDBASE prior, selecting other RBD main menu items. A flowchart of the data flow through RBD when operated in the EASY mode is shown in Fig. 15. In the flow chart, RBD programs are shown in bold and data bases and records files are shown in italics. The flowchart assumes that the dosimetry account code is DEMO. 


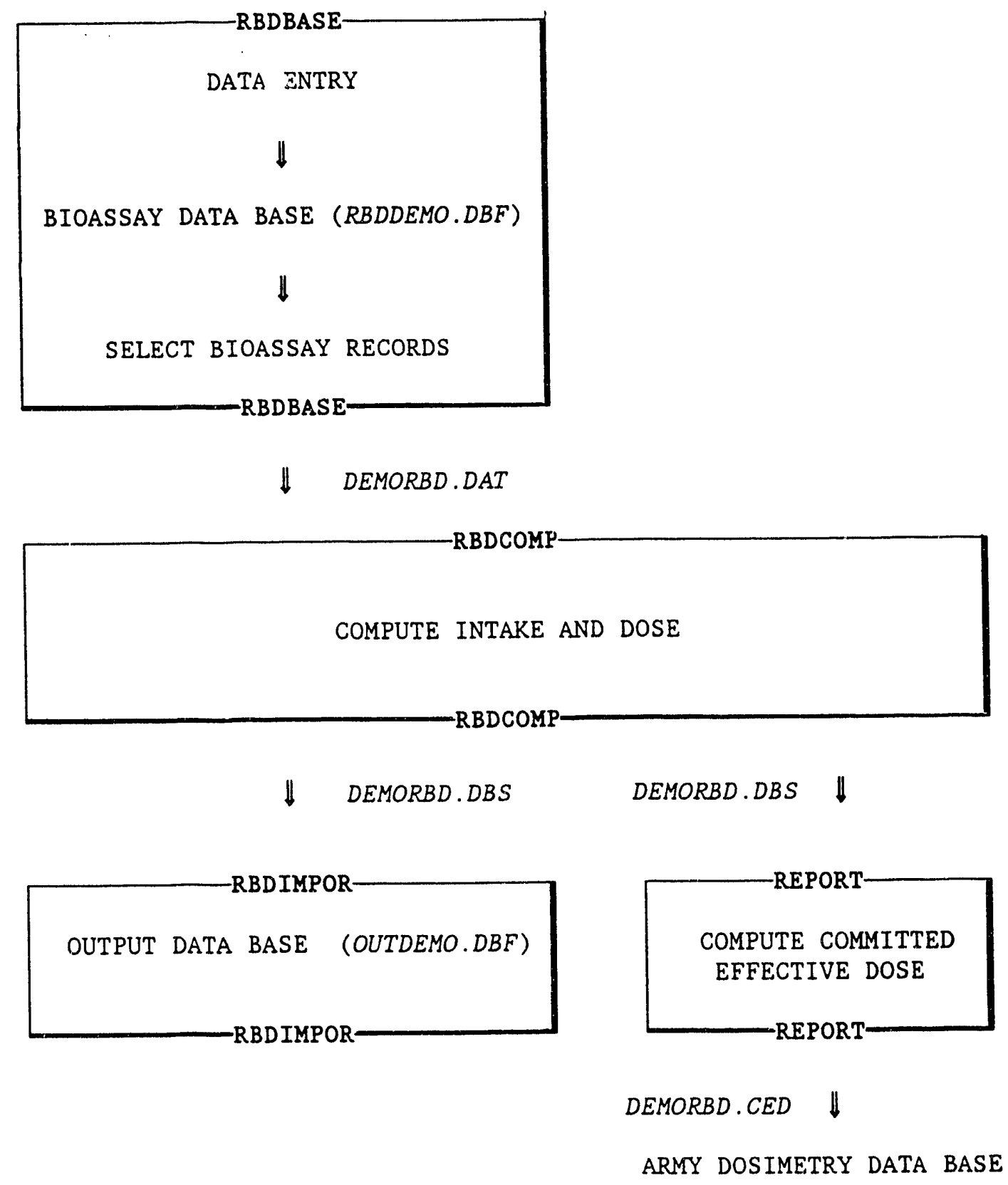

Fig. 15. Data flow through the RBD software package when in the EASY mode of operation. This assumes that the dosimetry account code is DEMO. RBD programs are shown in bold, and data bases and records files are shown in italics. 
The RBD main menu will indicate when the EASY mode has been selected (see below).

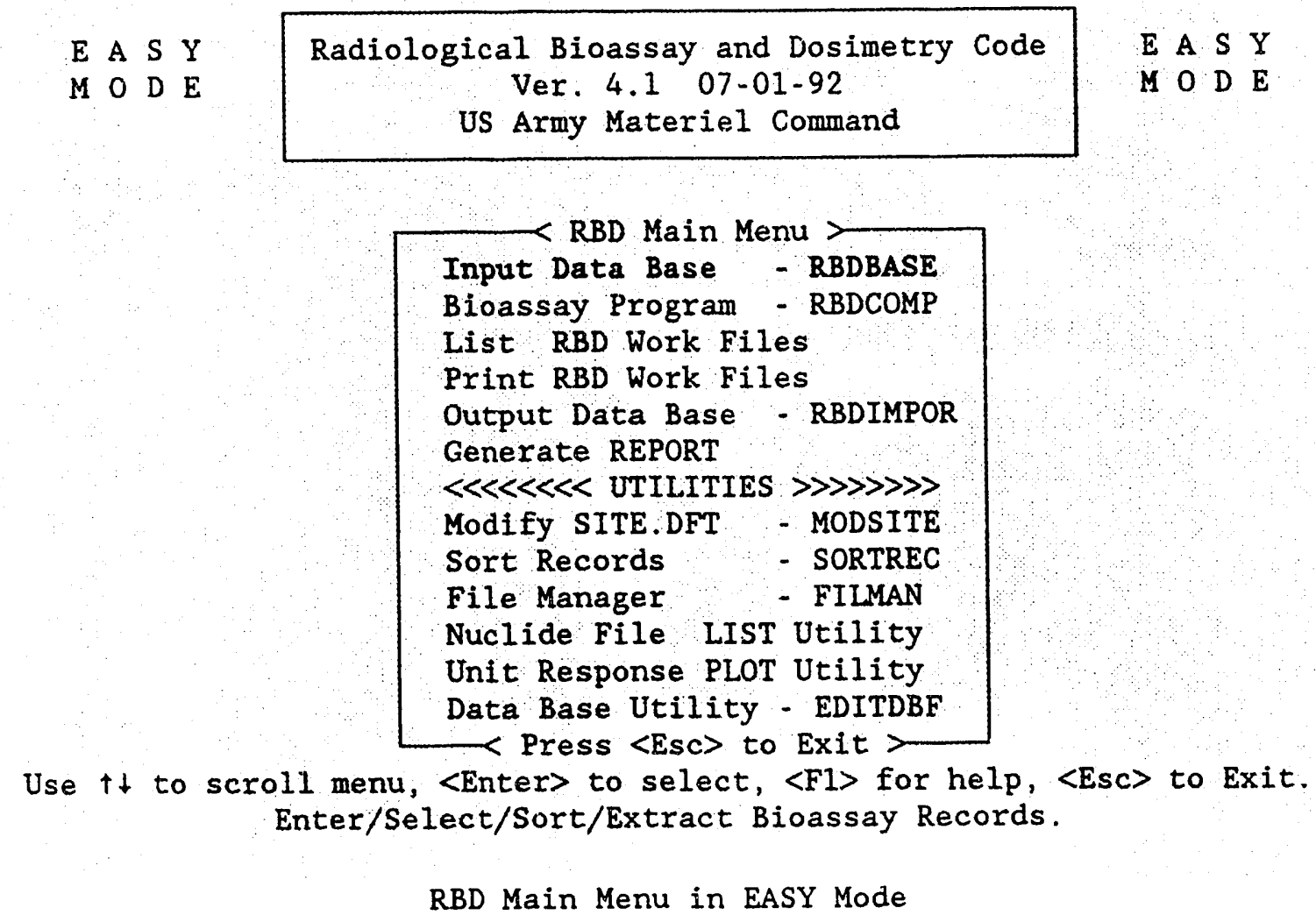

The bioassay data base (RBDDEMO.DBF) is opened whenever the user selects Input Data Base RBDBASE from the RBD main menu. The appearance of the RBDBASE menu is unaltered and the actions of the RBDBASE menu are the same as for the ADVANCED mode, with one exception. When the user selects bioassay records for input to RBDCOMP, the program creates the DAT file name. The filename is created by appending " $R B D$ " to the dosimetry account code. Assuming that the dosimetry account code is DEMO, the bioassay records filename is DEMORBD.DAT. As with the ADVANCED mode, the filename for the output records is created by adding the extension DBS to the bioassay records filename (DEMORBD.DBS). You have no choice over the names of these intermediate files and never have to select a DAT file for input to RBDCOMP or a DBS file for input to REPORT. This eliminates some of the confusion in operating RBD, but also reduces the versatility of the software. In particular, you will need to archive the RBD working files, if you wish to save them, after each run, because the RBDCOMP program will erase the previous working files.

A simplification is introduced when running the RBDCOMP and REPORT programs in the EASY mode. The display option is set when the EASY mode is specified by SETUPRBD. The same display mode is used by both codes. This means that, if you want to run RBDCOMP in batch mode (display option 0), but want to modify assay-weighting factors when REPORT runs (display option 1), you must enter display option 1 in the RBD SETUP menu. 
When the RBDCOMP program completes, the output records are immediately loaded into the output data base (OUTDEMO.DBF). The user can no longer import output records files (DBS files) by selecting the Output Data Base - RBDIMPOR menu item. However, this menu item can still be used to browse the output data base and to determine which bioassay records were used to compute the marked outpus records. Thus, the RBDIMPOR menu (see below) is modified in EASY mode only to the extent that the first item, the import feature, has been disabled.

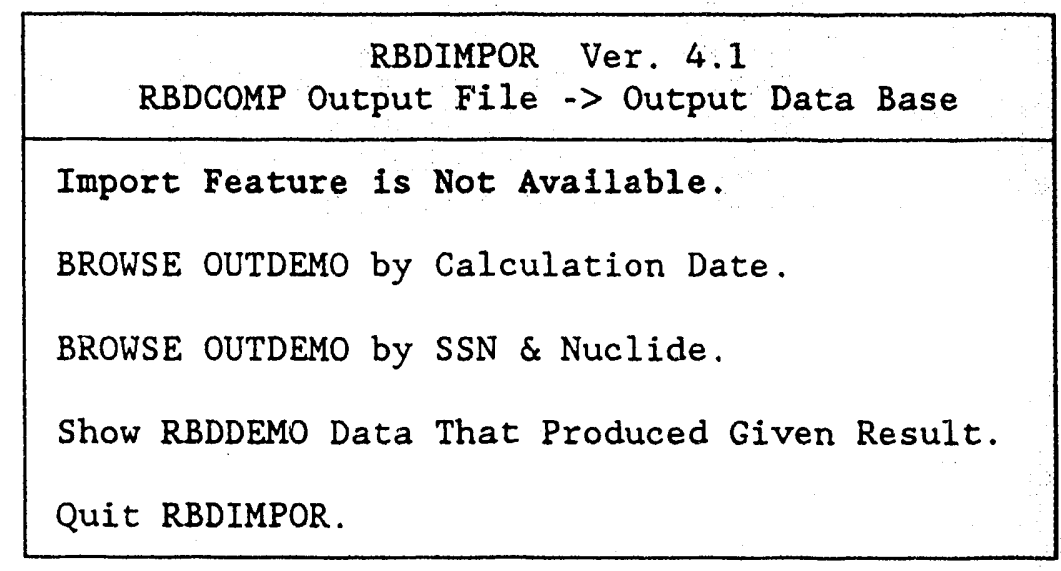

Data bases for this session are OUTDEMO and RBDDEMO.

The import feature is not available when EASY mode is specified.

\section{RBDIMPOR Menu in EASY Mode}

When you select Generate REPORT from the RBD main menu, you have no choice as to the DBS file for input; the file DEMORBD.DBS is automatically assumed. Finally, the SORTREC Utility, which is needed only when multiple DAT or DBS files must be appended and sorted, has been disabled in the EASY mode.

Thus the actions of five RBD main menu items are altered in EASY mode: Input Data Base RBDBASE, Bioassay Program - RBDCOMP, Output Data Base - RBDIMPOR, Generate REPORT, and Sort Records - SORTREC. The seven other menu items function exactly as in the ADVANCED mode described in the body of the report. 


\section{RBD EASY Mode Tutorial}

The following tutorial has been developed to demonstrate how RBD operates in the EASY mode. You should begin at the DOS prompt in the RBD directory.

Type SETUPRBD at the prompt. Verify that the dosimetry account code is DEMO. Change the mode of operation from $\operatorname{ADVANCED}(A)$ to $\operatorname{EASY}(E)$. Enter display option 1 for RBDCOMP and quit SETUPRBD. At the DOS prompt type RBD and press $<$ Enter $>$. Press <Enter > again to clear the RBD title screen. You will then see the RBD main menu with indications, on either side of the header, that you are in EASY mode.

Now select the item Input Data Base - RBDBASE. This will create the bioassay data base (RBDDEMO.DBF) and the output data base (OUTDEMO.DBF). At this point the data bases are empty; this can be confirmed for the bioassay data base by selecting the BROWSE menu item.

Normally, one would now select Add Data to Data Base to enter bioassay data. To speed up the tutorial, an existing bioassay records file (DAT file) supplier with the RBD installation will be loaded into the bioassay data base using AUTOLOAD.

Select Run AUTOLOAD Utility. From the list of bioassay records files (DAT files) shown, select H3.DAT. This is an incident exposure to tritium vapor. Press <Enter $>$ to mark this file and move the menu bar to Process Files and press <Enter $>$. Respond Yes to proceed to add these bioassay records to the RBDDEMO.DBF data base. Press $<$ Enter > to exit the AUTOLOAD utility. Select Browse RBDDEMO Data Base from the RBDBASE menu, to confirm the records are there. Press $<$ Esc $>$ to exit and return to RBDBASE menu. 
Now, select RBDBASE menu item Select Input for RBDCOMP. Select ALL DATA. Note that you are not asked for a name for the bioassay records file; the file is automatically named DEMORBD.DAT. Quit RBDBASE to return to the RBD main menu.

To process the chosen bioassay records and load the output records into the output data base, you select Bioassay Program - RBDCOMP from the RBD main menu. Note that in EASY mode you do not select the bioassay records file (DAT file), this is assumed to be DEMORBD.DAT. In addition, RBDCOMP uses the display option specified by SETUPRBD. You must re-run SETUPRBD to change the display option. You will continue to enter the intake period, and, if routine measurements are present, the measurement period, as was done with the ADVANCED mode of operation.

Select Bioassay Program - RBDCOMP from the RBD main menu. Enter an intake year of 1991 with a duration of 1 year.

The results of the computation, as displayed on the screen, are shown below.

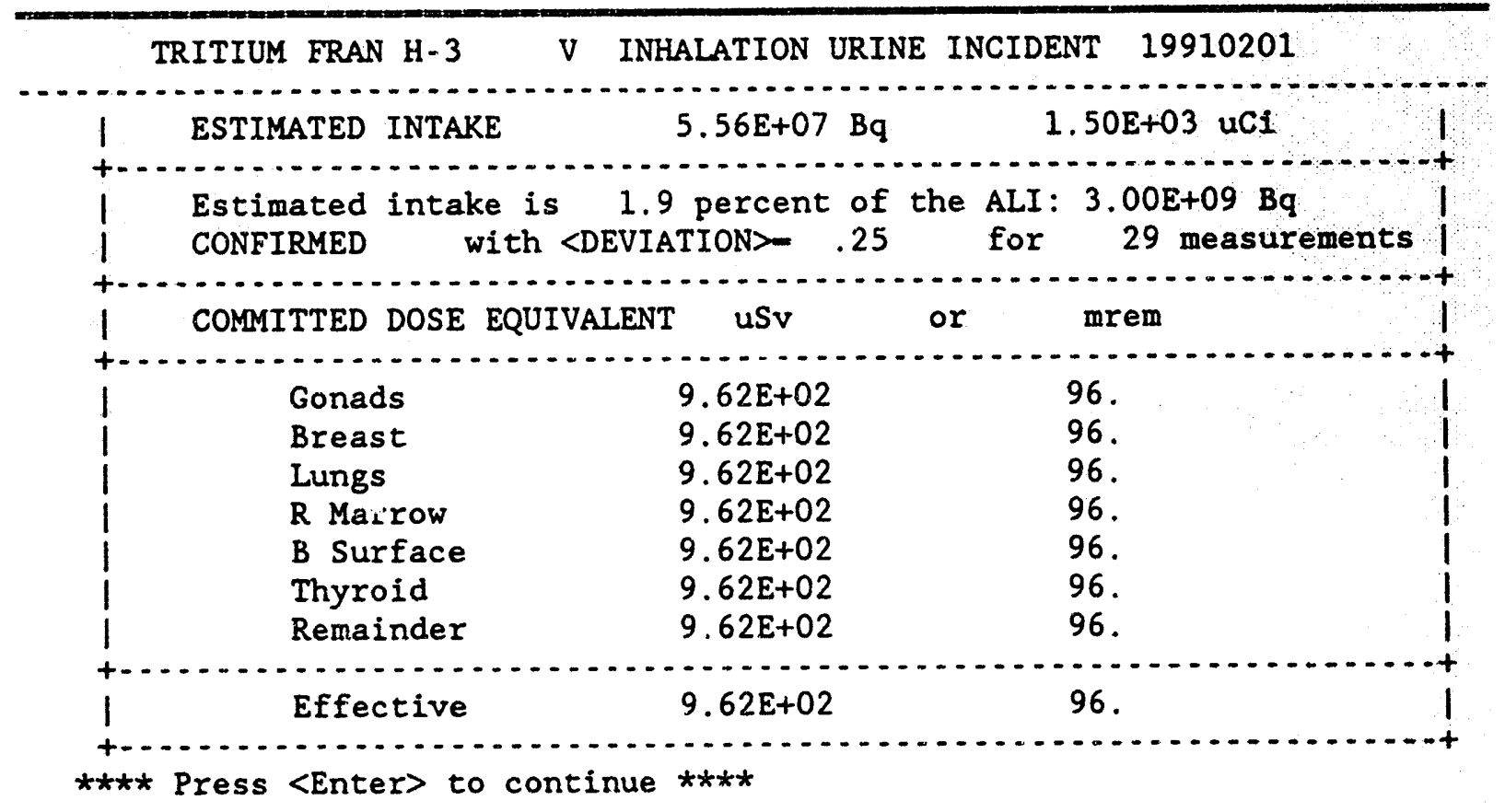


Respond Yes to Plot measurements per unit intake and unit response function? Accept the default minimum and maximum values.

The resulting plot is shown in Fig 16. At the conclusion of RBDCOMP (or REPORT, if this is set to run immediately after RBDCOMP), the following statement is seen on the screen:

RBDCOMP output records were imported into OUTDEMO data base,

indicating that the output records have been placed in the output data base.

Now select Output Data Base - RBDIMPOR from the RBD main menu. Note that the import feature has been disabled in EASY mode. Select Browse OUTDEMO by SSN \& Nuclide to examine the output records.

You will see a single output record, as shown below.

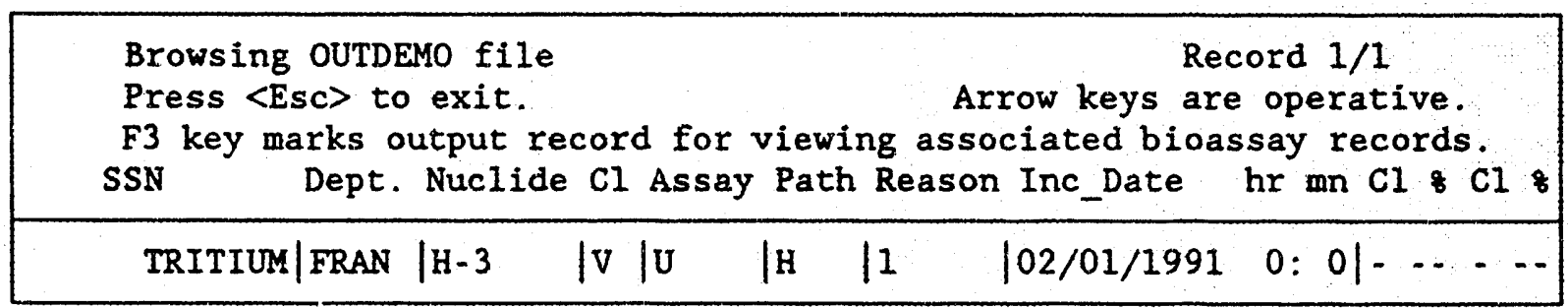

You can browse the bioassay records used to compute this output record by pressing $\langle$ F3 $\rangle$, exiting the output data base by pressing <Esc >, and selecting Show RBDDEMO Data That Produced Given Result.

Press <Esc> to exit the Browse feature and select Quit RBDDMPOR to return to the RBD main menu. 


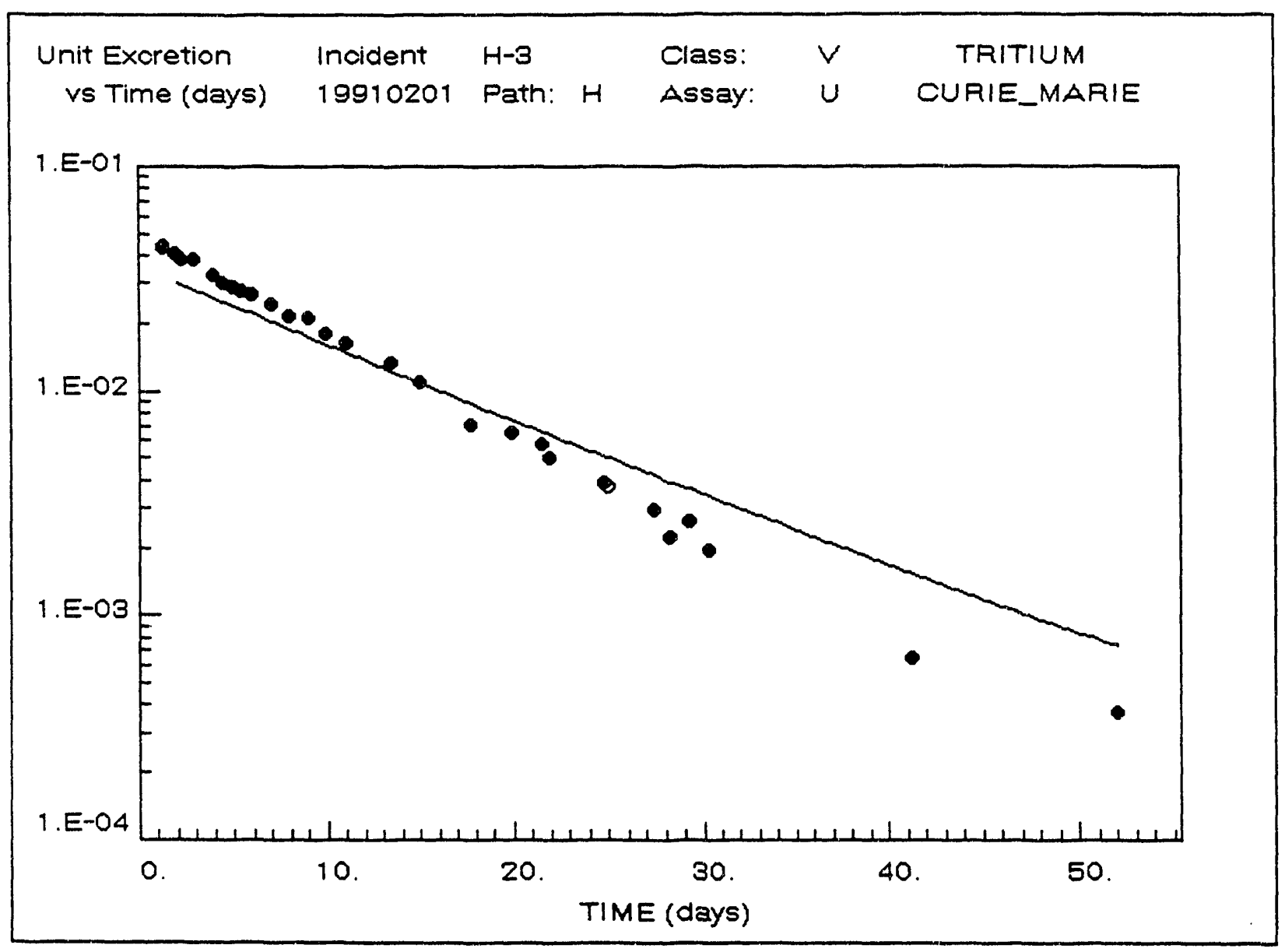

Fig. 16. Observed and expected urinary excretion for acute exposure to ${ }^{3} \mathrm{H}$ vapor. 
Three other RBD main menu items behave differently in the EASY mode of operation. The item Generate Report will use the output records file (DEMORBD.DBS in this tutorial) generated by RBDCOMP, to compute the committed effective dose equivalent for each individual. The menu item Sort Records - SORTREC is disabled and the Data Base Utility - EDITDBF will automatically select the single bioassay and output data bases available in EASY mode.

Select Sort Records - SORTREC from the RBD main menu to demonstrate that if it is disabled. Select Data Base Utility - EDITDBF and note that, since the dosimetry account code is $D E M O$, the bioassay data base will be RBDDEMO and the output data base will be OUTDEMO. Browse both of these data bases and then quit RBDIMPOR to return to the RBD main menu. This concludes the RBD EASY mode tutorial.

To return RBD to the ADVANCED mode, press <Esc > to exit the RBD main menu and type $S E T U P R B D$ at the DOS prompt. Replace $E$ with $A$ when asked for Easy of Advanced Mode? After concluding this tutorial, you should use SETUPRBD to change the dosimetry account code from $D E M O$ to that for your facility. If you should continue to use DEMO as the dosimetry account code and to operate in the EASY mode, you may wish to remove the records you have just entered into the bioassay and output data bases. Use the EDITDBF feature to zap, i.e. remove, the contents of the data bases.

The EASY mode of operation was created in response to suggestions at the Internal Dosimetry Training Course held at Ft. Belvoir from July 13-17, 1992. We wish to thank those at the course for the suggestions they made with regards to improving the ease of use of RBD. The EASY mode should greatly simplify use of the RBD software in an environment where multiple bioassay data bases are not required. The code developers welcome comments and suggestions about the RBD software. These can be made using the RBD ERROR REPORT found on page 9. 
ORNL/TM-11858

\section{INTERNAL DISTRIBUTION}
1-3. J. M. Barber
4. B. A. Berven
5. R. A. Boys
6-8. E. M. Brackett
9. M. Cristy
10. O. W. Cypret
11. E. Dixon
12-14. J. K. Duncan
15. C. E. Easterly
16-20. K. F. Eckerman
21-23. D. Farver
24. S. V. Kaye
25. G. D. Kerr
26. D. C. Kocher
27. R. W. Leggett
28. P. B. Lowe
29. P. Y. Lu
30. J. M. Mahathy
31. C. E. Maples

32. D. A. McLaughlin

33. G. L. Murphy

34. P. S. Rohwer

35. J. C. Ryman

36-38. C. H. Shappert

39. C. S. Sims

40. A. L. Sjoreen

41. M. Thein

42. J. F. Thompson

43. E. R. Wagner

44. G. E. Whitesides

45-46. R. C. Ward

47. Central Research Library

48. ORNL Y-12 Research Library

Document Reference Section

49. Laboratory Records Department

50. Laboratory Records, ORNL (RC)

51. ORNL Patent Office

\section{EXTERNAL DISTRIBUTION}

52. A. E. Abney, Chief, USAIRDC, ATTN: AMXTM-S-LR-D, Lexington, KY 405115102

53. C. L. Adams, U. S. Army, Engineer District, KC, ATTN: CEMRK-ED-GE, $601 \mathrm{E}$. 12th Street, Kansas City, MO 64106-2896

54. J. A. Auxier, Auxier \& Associates Inc., Suite 500, 111 Marby Hood Rd., Knoxville, TN 37922

55. W. J. Bair, Manager, Environment, Health and Safety Research Program, Battelle Pacific Northwest Laboratories, P. O. Box 999, Richland, WA 99352

56. L. V. Bender, Commander, U. S. Army BRDEC, ATTN: STRBE-VR, Fort Belvoir, VA $22060-5606$

57. L. Bertelli, Environmental Radiation \& Toxicology Laboratory, School of Medicine, 1771 So., 900 W., Suite 10, Salt Lake City, Utah 84104 
58. R. K. Bhat, U. S. Army Belvoir, RD\&E Center, STRBE-VR, Fort Belvoir, VA 22060

59. Arden E. Bicker, Assistant Manager Environmental \& Health Division, P. O. Box 98521, Las Vegas, NV 89193-8521

60. Paul Black, U. S. Army Materials, Technology Lab, 405 Arsenal Street, Watertown, MA 02172-0001

61. Troy Blanton, NAVSEADET-RASO, Yorktown, VA 23691

62. Annette Boatwright, U. S. Army Environmental Hygiene Agency, ATTN: HSHBMR-H, Aberdeen Proving Ground, MD 21010-5422

63. B. B. Boecker, Inhalation Toxicology Research Institute, Lovelace Biomedical and Environmental Research Institute, P. O. Box 5890, Albuquerque, NM 87185

64. A Bouville, National Cancer Institute, Radiation Effects Branch, Executive Plaza North, Suite 530, Bethesda, MD 20892

65. M. W. Bower, USAEHA, ATTN: HSHB-MR-HM, Aberdeen Proving Ground, MD 21010

66. Debbie Bulter, Belvoir RD\&E Center, ATTN: STRBE-TVR (D. Bulter), Fort Belvoir, VA 22060-5606

67. T. W. Carlen, Sierra Army Depot, SDSSI-DSW, Herlong, CA 96113-5500

68. R. S. Caswell, U. S. Department of Commerce, NIST, Bldg. 245/C-229, Gaithersburg, MD 20899

69. R. Chastain, Sierra Army Depot, SDSSI-DSW, Herlong, CA 96113-5500

70. X. Chen, Radiation Health Division, Laboratory of Industrial Hygiene, Ministry of Public Health, 2 Xinkang Street, Deshengmenwai, P. O. Box 8018, Beijing 100088, People's Republic of China

71. J. S. Clark, U. S. Department of Commerce, NIST, Bldg. 235/A132, Gaithersburg, MD 20899

72. Jan Clemons, Commander, U. S. Army Missile Command, ATTN: AMSMI-IM-ADCA-SA, Redstone Arsenal, Huntsville, AL 35898-5173

73. A. B. Cooper, U. S. MEDDEC, Fort Hood, TX 76544-5063

74. Subinoy Das, Belvoir RD\&E Center, ATTN: STRBE-TVR (S. Das), Fort Belvoir, VA 22060-5606 
75. J. S. Davis, EAMC, ATTN: HSHF-HP, Health Physics Office, Fort Gordon, GA 30905-5605

76. L. S. Davis, U. S. Army Combat Systems, Test Activity, STECS-SO, Aberdeen Proving Grounds, MD 21005-5059

77. LTC C. A. Day, U. S. Army Materiel Command HQ AMC, ATTN: AMCSG-R, 5001 Eisenhower Avenue, Alexandria, VA 22333-0001

78. L. T. Dillman, 184 W. Lincoln Ave., Delaware, OH 43015

79. David Dinh, Belvoir RD\&E Center, ATTN: STRBE-TVR (D. Dinh), Fort Belvoir, VA 22060-5606

80. MAJ S. Dunston, USAEHA, ATTN: HSHB-MR-H, Aberdeen Proving Ground, MD 21010-5422

81. P. A. Elker, U. S. Army Materiel Command, 5001 Eisenhower Avenue, ATTN: AMCSF-P, Alexandria, VA 22333-0001

82. F. M. Fota, USAEHA, ATTN: HSHB-MR-HM, Aberdeen Proving Ground, MD $21010-5422$

83. Michael Furkkhouser, Belvoir RD\&E Center, ATTN: STRBE-TVR (M. Funkhouser), Fort Belvoir, VA 22060-5606

84. Alexander J. Guza, Commanding Officer (B360), Marine Corps Logistics Base, Barstow, CA 92311-5015

85. Kimberly A. Hager, Ionizing Radiation, Dosimetry Center, ATTN: AMXTM-S-LR-D, Lexington, KY 40511-5102

86. N. H. Harley, Department of Environmental Medicine, New York University Medical Center, 550 First Avenue, New York NY. 10016

87. S. W. Holmes, AFRRI, Bldg. 42 NNMC, ATTN: SHDH, Bethesda, MD 20889

88. J. L. Hosszu, NAVSEADET-RASO, Yorktown, VA 23691

89. C. E. Iranzo, Paseo de la Castellana 201, Madrid 28046, Spain

90. Craig E. Jensen, Radiological Safety Officer, 505 King Avenue, Columbus, OH 43201-2693

91. John C. Johnson, HQAMC, ATTN: AMCSG-R, 5001 Eisenhower Avenue, Alexandria, VA 22152 
92. Emma L. Kephart, DNA, AFRRI, Bldg. 42 South Palmer Road, Bethesda, MD 20889-5145

93. Andy Kung, U. S. Army ARDEC, ATTN: SMCAR-SF, Bldg. 320, Picatinny Arsenal, NJ 07806-5000

94. Michael R. Lewis, SENECA Army Depot, ATTN: SDSSE-LS, Romulus, NY 145415001

95. Gee-Fong Liang, Radiation Laboratory, Taiwan Power Company, P. O. Box 7, Shinmen, Taiwan 253, Republic of China

96. I. A. Likhtarev, Ukraine Scientific Centre for Radiation Medicine, Melnikova Street 53, 252050 Kiev-50, Ukraine

97. Richard Lovell, CDR CECOM, ATTN: AMSEL-SF-RER, Fort Monmouth, NJ 07703-5024

98. LTC Larry W. Luckett, Department of Physics, U. S. Military Academy, West Point, NY 10996-1790

99. Glenn Lykkes, Physics Department, University of ND, Box 8008 University Station, Grand Forks, ND 58202

100. Richard A. Markland, U. S. Army, Ballistic Research Laboratory, ATTN: SLCBRDD-H, Aberdeen Proving Grounds, MD 21005-5066

101. SFC Thomas N. Marsh, Health Physics Office Building 188, FGS, Walter Reed AMC, Washington, DC 20307-5001

102. John W. May, UAS CMLS, ATTN: ATZN-CM-AHP, Fort McClellan, Al 36205 . 5020

103. Mark L. Mays, UASF, 2750 ABW/EMB, Wright Patterson AFB, OH 45433

104. Michael D. McKinnon, Armstrong Lab/OEBSA, Brooks AFB, TX 78235-5000

105. C. B. Meinhold, Chief, Safety and Environmental Protection Division, Bldg. 525A, Brookhaven National Laboratory, Upton NY 11973

106. H. Metivier, CEA - Centre d'Etudes de Bryeres le Chatel, B. P. No. 12, 91680 Bruyeres le Chatel, France

107. H. Timothy Mikulski, Belvoir RD\&E Center, ATTN: STRBE-TVR (T. Mikulski), Fort Belvoir, VA 22060-5606

108. W. A. Mills, 2915 Ascott Lane, Olney, MD 20832 
109. Dr. Richard Moore, U. S. Department of Energy, 785 DOE Place, Mail Stop \# 4149, Idaho Falls, ID 83402-4149

110. Arthur Ray Morton III, USAEHA, ATTN: HSHB-MR-HM, Aberdeen Proving Grounds, MD 21010-5422

111. C. B. Nelson, U. S. EPA Office of Radiation Programs, 401 M. Street, S. W., Mail Code 6602J, Washington, DC 20460

112. J. C. Nenot, Institut de Protection et de Surete Nucleaire, Department de Protection, Services de Pathologie et de Protection Saitaire, Service de Protection Sanitaire, Centre d'Etudes Nucleaires, B. P. No. 6, 92260 Fontenay-aux-Roses, France

113. Ronald Nimitz, Nuclear Regulatory Commission, 475 Allendale Road, King of Prussia, PA 19406-5606

114. Thomas J. O'Brien, AFRRI, Bldg. 42, ATTN: SHDH, NNMC, Bethesda, MD 20814

115. Dr. Nicole Parmentier, Nuclear Protection and Safety Institut CEA B. P. 6-92265, Fontenay AUX Roses Cedex, France

116. W. Scott Pennington, USNRC, Mail Stop 6H3, Washington, DC 20555

117. Robert D. Pierce, NASA, Ames Research Center, Mail Stop T-036A, Moffett Field, CA $94035-1000$

118. J. W. Poston, Department of Nuclear Engineering, College of Engineering, Texas A\&M University, College Station, TX 77843

119. William Pramenko, USAF, Armstrong Laboratory, OEBSA, Brooks, AFB, TX 78235

120. P. V. Ramzaev, Institute of Radiation Hygiene, 8 Mira ul, St. Petersburg 19701, Russia

121. A. C. B. Richardson, 401 M. Street, S. W., Mail Code 6602J, Washington, DC 20460

122. C. T. Raddatz, Nuclear Regulatory Commission, MS NLS-139, Washington, D.C. 20555

123. Gene Runkle, U. S. Department of Energy, Albuquerque Operations, Environmental Safety and Health Division, Albuquerque, NM 87115

124. E. L. Saenger, Eugene L. Saenger Radioisotope Laboratory, University of Cincinnati Hospital, Cincinnati General Division, 234 Goodman Street, Cincinatti, OH 45267

125. T. Schlesinger, Soreq Nuclear Center, Yarneh, Isreal 70600

126. K. W. Skrable, University of Lowell, Lowell, MA 01854 
127. L. A. Slaback, U. S. Department of Commerce, NIST, Bldg. 245/C125, Gaithersburg, MD 20899

128. Calvin J. Slotemaker, Puget Sound Naval Shipyard, Code 105.5, Bremerton, WA 98314

129. CPT Henry Snyder, Walter Reed Army Medical Center, Building 188, Forest Glen Section, Washington, DC 20307-5001

130. Sean Soong, US NRC, Mail Stop 6H3, Washington, DC 20555

131. M. T. Stabin, Radiopharmaceutical Internal Dose Center, Oak Ridge Associated Universities, MERT, P.O. Box 117, Oak Ridge, TN 37831

132. Charlotte L. Stanfield, Ionizing Radiation, Dosimetry Center, ATTN: AMXTM-SLR-D, Lexington, Ky 40511-5102

133. J. W. Stather, National Radiological Protection Board, Chilton, Didcot, Oxon, OX11 ORQ, U. K.

134. C. Sun, Radiological Sciences Division, Department of Nuclear Energy, Brookhaven National Laboratory, Building 703M, Upton, NY 11973

135. Karl Swartz, USA IRDC, ATTN: AMXTM-CE-DC, Lexington, KY 40511-4102

136. R. J. Swatski, USAEHA, ATTN: HSHB-ML-RR, Aberdeen Proving Ground, MD 21010-5422

137. D. M. Taylor, Kernforschungszentrum Karlsruhe, Institute for Genetics and Toxicology, Postfach 3640, D-7500 Karlsruhe 1, Federal Republic of Germany

138. James H. Terry, U. S. Department of Commerce, NIST, Gaithersburg, MD 20899

139. Mark G. Tiedemann, AFSC Regional Hospital/SGPB, Eglin AFB, FL 32542

140. Juan A. Torres, USACMLS, ATTN: ATZN-CM-AHP, Fort McCLellan, AL 362055020

141. James W. Tracy, U. S. Department of Commerce, NIST, Gaithersburg, MD 20899

142. B. W. Wachholz, Low Level Radiation Effects Branch, National Cancer Institute, Landow Building 8C-09, 9000 Rockville Pike, Bethesda, MD 20205

143. E. E. Watson, Radiopharmaceutical Internal Dose Center, Oak Ridge Associated Universities, MERT, P. O. Box 117, Oak Ridge, TN 37831

144. William R. Webber, U. S. Department of Commerce, NIST, Building 245/C125, Gaithersburg, MD 20899 
145. D. A. Weber, Brookhaven National Laboratory, Medical Department Building 490, Upton, NY 11973-5000

146. L. R. Williams, Indiana University at South Bend, Mathematics Department, 1700 Mishawaka Avenue, South Bend, IN 46634

147. C. D. Willson, Department of Radiation Biology, Medical College of St Bartholomew's Hospital, University of London, Charterhouse Square, London EC1M 6BQ

148. Ben Wilmoth, 2750 ABW/EMB, WPAFB, OH 45433

149. B. C. Winkler, Private Bag X256, Pretoria, 0001, South Africa

150. R. W. Wood, Physical and Technology Research Division, ER-74, Office of Health and Environmental Research, U. S. Department of Energy (GTN), Washington, DC 20545

151. W. A. Woolson, Science Applications Incorporated, 1200 Prospect Street, P. O. Box 2351, La Jolla, CA 92038

152. M. E. Wrenn, Environmental Radiation \& Toxicology Laboratory School of Medicine, 1771 So., 900 W., Suite 10, Salt Lake City, Utah 84104

153. Robert A. Wynvean, Argonne National Laboratory, 9700 S. Cass Avenue, Argonne, IL 60439

154. Shlomo Yaniv, Health Effects Branch - MS 1130-SS, Division of Health, Siting and Waste Management, RES, U. S. Nuclear Regulatory Commission, Washington, DC 20555

155. R. W. Young, Armed Forces Radiobiology Research Institute, Naval Medical Center, Bethesda, MD 20814-5145

156. Mark Zarick, CDR CECOM, ATTN: AMSEL-SF-RER, Fort Monmouth, NJ 077035024

157. Gavin Ziegler, HQ AMCCOM, ATTN: AMSMC-SFS (G. Ziegler), Rock Island, IL $61299-6000$

158. Office of the Assistant Manager for Energy, Research and Development, U. S. Department of Energy Field Office, Oak Ridge (DOE-OR), P. O. Box 2001, Oak Ridge, TN 37831

159-169. Office of Scientific and Technical Information, P. O. Box 62, Oak Ridge, TN 37831 

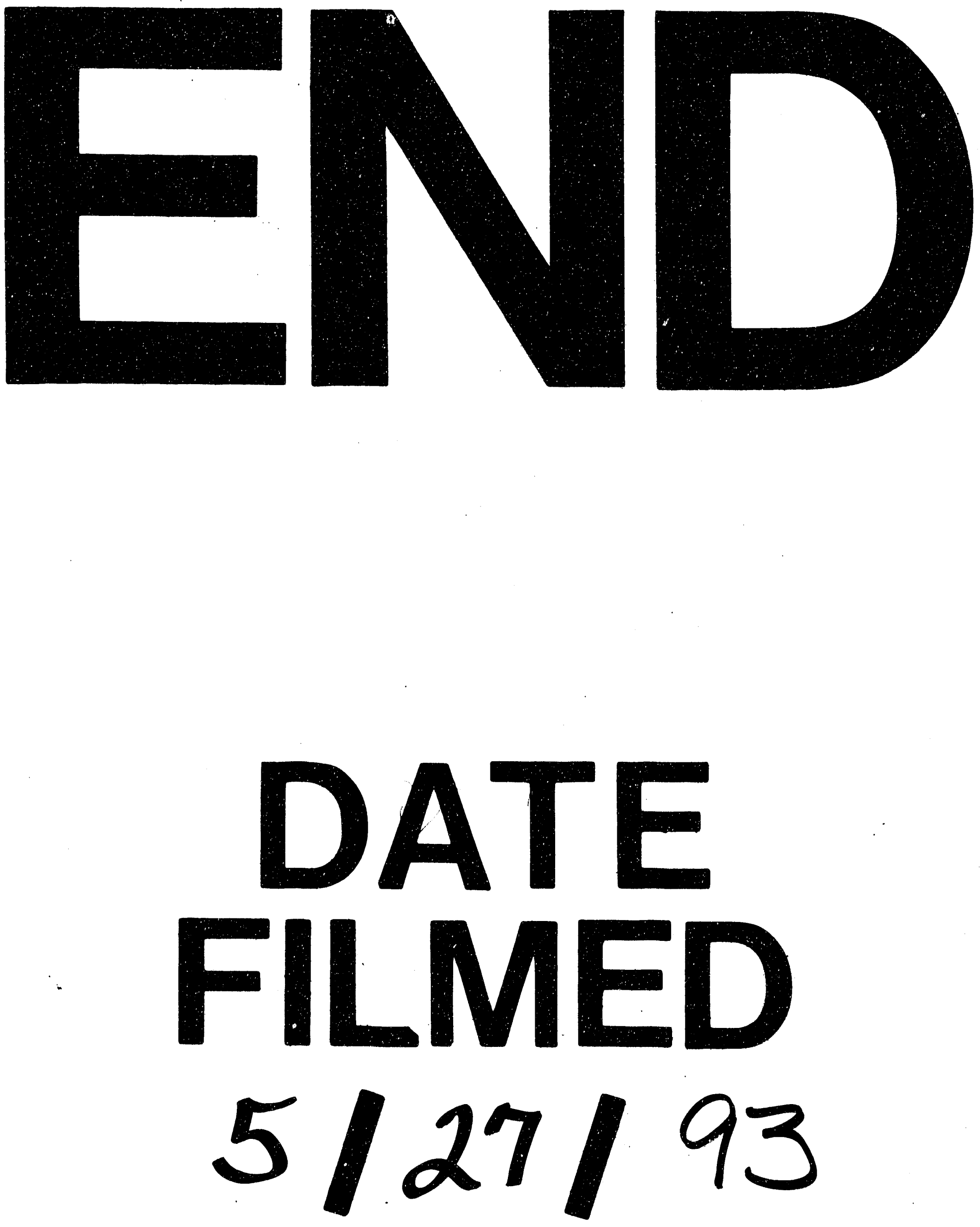
